\title{
FOREST STRUCTURAL COMPLEXITY IN A TEMPERATE HARDWOOD FOREST: A GEOMATICS APPROACH TO MODELLING AND MAPPING INDICATORS OF HABITAT AND BIODIVERSITY
}

\author{
By \\ Jonathan Pasher, M.Sc. \\ A thesis submitted to \\ The Faculty of Graduate Studies and Research \\ in partial fulfillment of the requirements for the degree of
}

Doctor of Philosophy

Department of Geography and Environmental Studies

Carleton University

Ottawa, Ontario

July, 2009

(C) Jonathan Pasher 


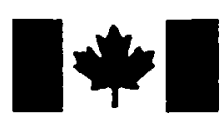

\author{
Library and Archives \\ Canada \\ Published Heritage \\ Branch \\ 395 Wellington Street \\ Ottawa ON K1A ON4 \\ Canada
}

Bibliotheque et

Archives Canada

Direction du

Patrimoine de l'édition

395 , rue Wellington

Ottawa ON K1A ON4

Canada
Your file Votre référence
ISBN: $978-0-494-60105-1$
Our file Notre référence
ISBN: $978-0-494-60105-1$
NOTICE:

The author has granted a nonexclusive license allowing Library and Archives Canada to reproduce, publish, archive, preserve, conserve, communicate to the public by telecommunication or on the Internet, loan, distribute and sell theses worldwide, for commercial or noncommercial purposes, in microform, paper, electronic and/or any other formats.

The author retains copyright ownership and moral rights in this thesis. Neither the thesis nor substantial extracts from it may be printed or otherwise reproduced without the author's permission.
AVIS:

L'auteur a accordé une licence non exclusive permettant à la Bibliothèque et Archives Canada de reproduire, publier, archiver, sauvegarder, conserver, transmettre au public par télécommunication ou par l'Internet, prêter, distribuer et vendre des thèses partout dans le monde, à des fins commerciales ou autres, sur support microforme, papier, électronique et/ou autres formats.

L'auteur conserve la propriété du droit d'auteur et des droits moraux qui protège cette thèse. $\mathrm{Ni}$ la thèse ni des extraits substantiels de celle-ci ne doivent être imprimés ou autrement reproduits sans son autorisation.
In compliance with the Canadian Privacy Act some supporting forms may have been removed from this thesis.

While these forms may be included in the document page count, their removal does not represent any loss of content from the thesis.
Conformément à la loi canadienne sur la protection de la vie privée, quelques formulaires secondaires ont été enlevés de cette thèse.

Bien que ces formulaires aient inclus dans la pagination, il n'y aura aucun contenu manquant.

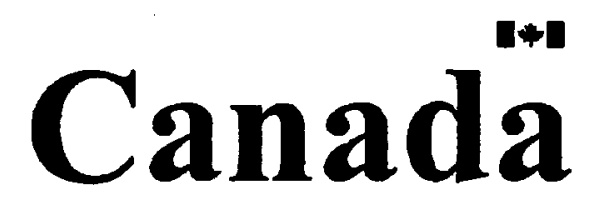




\begin{abstract}
Remote sensing has been widely used for modelling and mapping individual structural attributes within forests, however, knowledge of the multivariate nature of structural complexity, which is of specific interest as an indicator of forest habitat and biodiversity, is lacking. This research presents methods and results describing the development of geomatics-based indicators of forest structure, which are spatially continuous, extensive and repeatable. Two distinct, but related, structurally-based indicators derived from high resolution airborne imagery and topographic information were developed: (1) modelling and mapping forest structural complexity, and (2) the detection and mapping of the spatial distribution of dead wood. A Redundancy Analysis (RDA) was used to develop an image-based Structural Complexity Index (SCI) representing structural complexity as measured on the ground. An extensive set of image spectral, spatial, and object-based variables, along with topographic variables, were tested as predictors of structural complexity. The SCI, as a general gradient of structural complexity, accounted for $35 \%$ of the original variance in the field data. The model was applied spatially to map the SCI across the entire study area within Gatineau Park, Quebec. Field validation of the extreme conditions (high and low complexity areas) showed the map to be $\sim 80 \%$ accurate. Tests using simulated $60 \mathrm{~cm}$ and $1 \mathrm{~m}$ imagery showed potential for scaling up the RDA modelling procedure to be used with coarser resolution imagery, however map validation at these resolutions was somewhat inconclusive and further investigation using lower resolution airborne imagery and possibly high resolution satellite imagery is required. Additionally, a semi-automated method for detecting and mapping dead wood was investigated, with field validation of
\end{abstract}


detected objects having an accuracy of $94 \%$, and control sites, or areas with no detectable dead wood, showing an accuracy of $90 \%$. The methods presented in this research can help to advance remote sensing research for forest structure modelling and mapping, and specifically in a temperate hardwood forest. Further, they could potentially be adapted and applied to different forest types and used for enhancing forest inventories by providing methods for reporting on habitat and biodiversity levels. 


\section{ACKNOWLEDGEMENTS}

Research funding and equipment necessary for this project was provided by the National Sciences and Engineering Research Council, the Canada Foundation for Innovation, the Ontario Innovation Trust, the National Wildlife Research Centre, as well as private sources funding the Geomatics and Landscape Ecology Research Laboratory. Thanks to the NCC for providing data and most importantly access to Gatineau Park.

Doug, many thanks for recognizing my potential and for providing me with so many interesting and challenging opportunities over the years. I value your constant support, encouragement, and most importantly, your friendship.

Thank you to my thesis committee: Dr. Ian Olthof, Dr. Elyn Humphreys, and Dr. Jeremy Kerr. Your support and timely feedback throughout this process has been vital and much appreciated. Technical support provided by Nick Walsworth and Dan Patterson was greatly appreciated. Special thanks to Dr. Raphael Proulx for your endless support and advice with the modelling. Your enthusiasm and interest in this project has been wonderful. The fieldwork done for this project could never have been completed without the help of Chris, Michelle, Valerie, and Robby. Thanks for your hard work (and company) in the woods! Sorry about the bugs, but hope you had some fun!!

Mom, Dad, and all the family and friends that constantly surround me and contribute so much to my life, thank you for always supporting me and being, or at least pretending to be, interested!! Finally, thank you Tina for your endless support and encouragement and for putting up with the ups and downs of doing a PhD!! 


\section{TABLE OF CONTENTS}

ABSTRACT.................................................................................................................................. II

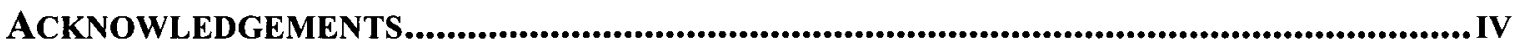

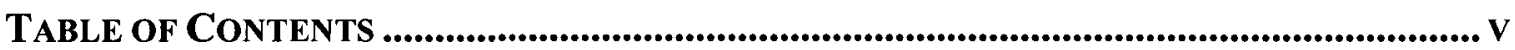

LIST OF TABLES................................................................................................................................ VIII

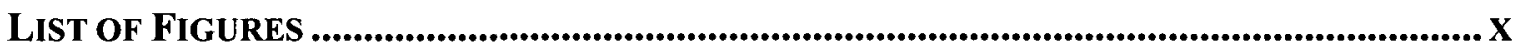

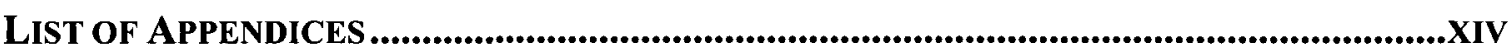

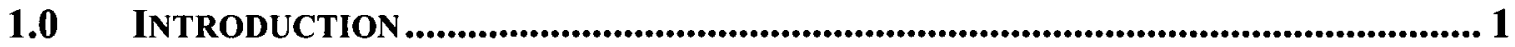

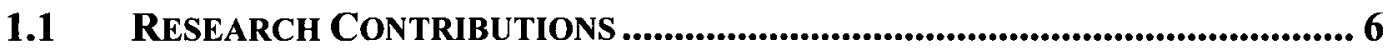

1.2 THESIS STRUCTURE ............................................................................ 8

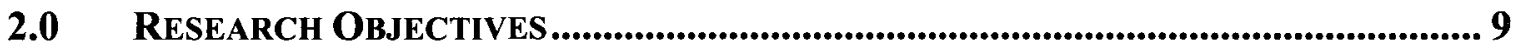

3.0 BACKGROUND............................................................................................................ 10

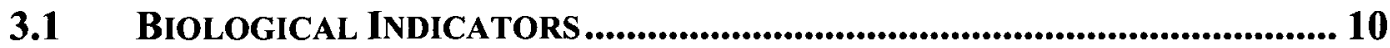

3.1.1 Species-Based Indicators of Biodiversity ................................... 10

3.1.2 Structural Indicators of Biodiversity............................................. 14

3.1.2.1 Keystone Structures Including Dead Wood .................... 15

3.1.2.2 Habitat Heterogeneity Hypothesis..................................... 17

3.1.2.3 Structural Complexity Indices ........................................ 19

3.2 MODELLING AND MAPPING BIODIVERSITY AND BIODIVERSITY

INDICATORS WITH REMOTE SENSING............................................................... 24

3.2.1 Indirect Modelling and Mapping of Species Distribution and Biodiversity ..................................................................... 24

3.2.2 Modelling Forest Structure with High Resolution Remote Sensing ....................................................................................... 29

3.2.3 Modelling and Mapping Dead Wood Using Remote

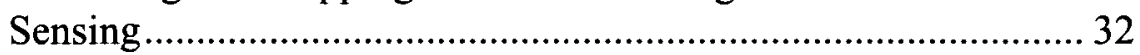

3.2.3.1 Direct Detection and Mapping ……………..................... 33

3.2.3.2 Indirect Modelling of Dead Wood .................................... 34

3.2.4 Structural Complexity Modelling Using Remote Sensing............ 36

$3.3 \quad$ JUSTIFICATION FOR THIS RESEARCH ....................................................... 40

4.0 METHODS..................................................................................................................... 43

4.1 STUDY AREA ....................................................................................................43 43

4.2 AIRBORNE IMAGERY COLlECTION AND PROCESSING............................... 45

4.3 DEVELOPMENT OF AN IMAGE-BASED STRUCTURAL COMPLEXITY

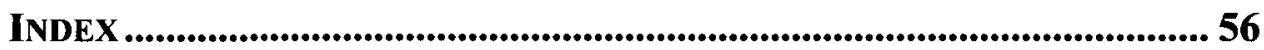

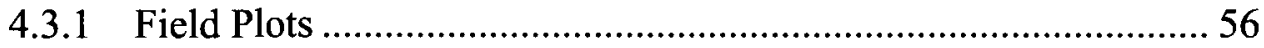


4.3.2 Structural Attribute Measurements .............................................5 57

4.3.2.1 Overstorey Tree Size and Distribution ............................. 57

4.3.2.2 Canopy Openness and LAI............................................59 59

4.3.2.3 Understorey and Ground Vegetation................................. 60

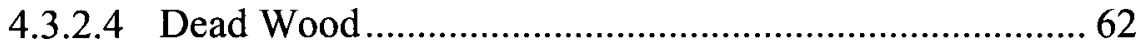

4.3.3 Explanatory / Predictor Variables ...................................................... 64

4.3.3.1 Image Spectral Variables..................................................65

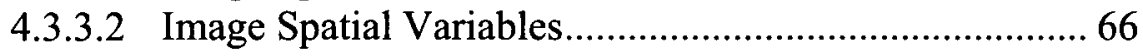

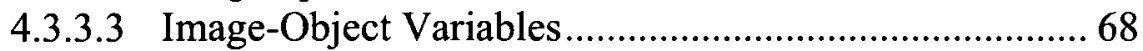

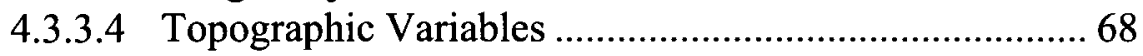

4.3.4 Modelling Structural Complexity Using Redundancy

Analysis............................................................................. 70

4.3.5 Mapping Structural Complexity ……………............................. 77

4.3.6 Structural Complexity Map Validation......................................... 79

4.3.7 Testing the Potential Scalability of the Methods .......................... 80

4.4 MODELLING AND MAPPING DEAD WOOD .................................................. 82

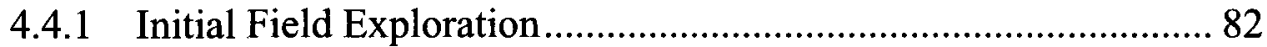

4.4.2 Remotely-Sensed Imagery …………………............................. 83

4.4.3 Direct Detection of Dead Wood in Imagery ................................. 84

4.4.3.1 ISODATA Unsupervised Clustering ............................... 85

4.4.3.2 Sub-pixel Wood Fractions using Unmixing ................... 86

4.4.3.3 Object-based Segmentation and Classification ............... 88

4.4.3.4 Developing a Map of Detected and Delineated

Wood Objects .................................................................. 89

4.4.3.5 Field Validation ..........................................................90

4.4.3.6 Map of Dead Wood Spatial Distribution........................ 92

4.4.4 Indirect Regression Modelling of Dead Wood ............................ 92

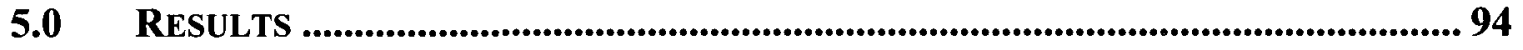

5.1 DEVELOPMENT OF AN IMAGE-BASED STRUCTURAL COMPLEXITY

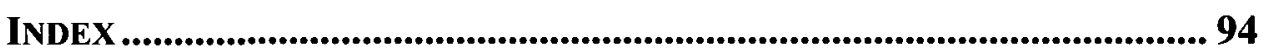

5.1.1 Field Variables Representing Structural Attributes ....................... 94

5.1.2 Image and Topographic Variables (Explanatory / Predictor Variables) ................................................................................ 100

5.1.3 Modelling Structural Complexity with Redundancy

Analysis............................................................................. 100

5.1.4 Final RDA Model ............................................................... 103

5.1.4.1 Interpreting the SCI from a Field Perspective.............. 108

5.1.4.2 Interpreting the SCI from a Remote Sensing

Perspective ................................................................ 112

5.1.5 Assessment of the RDA Model................................................ 116

5.1.6 Structural Complexity Mapping and Validation.......................... 117

5.1.7 Scalability of RDA Modelling and Mapping.............................. 124

5.1.7.1 RDA Modelling ........................................................ 124

5.1.7.2 Mapping Structural Complexity …………................... 127 
5.2 Modelling AND MAPPING DEAd WoOd ............................................. 130

5.2.1 Direct Detection of Dead Wood .............................................. 130

5.2.1.1 Dead Wood Map Validation....................................... 138

5.2.1.2 Assessment of Errors of Commission ......................... 138

5.2.1.3 Assessment of Errors of Omission ................................ 140

5.2.1.4 Mapping Dead Wood Spatial Distribution.................... 141

5.2.2 Indirect Modelling of Dead Wood.............................................. 142

5.3 COMBINING INDICATORS ........................................................................ 145

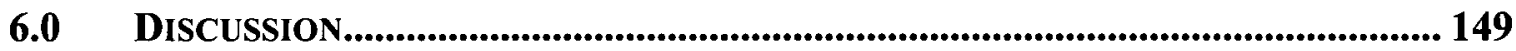

6.1 DEVELOPMENT OF AN IMAGE-BASED STRUCTURAL COMPLEXITY

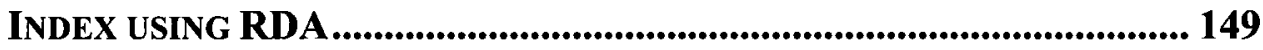

6.1.1 Model Scalability ................................................................... 153

6.1.2 Applicability of Results, Research Limitations, and Future

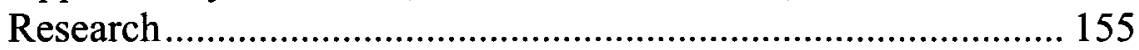

6.2 MODELLING AND MAPPING DEAD WOOD ................................................. 160

6.2.1 Direct Detection of Dead Wood .............................................. 160

6.2.2 Indirect Modelling of Dead Wood............................................... 161

6.2.3 Applicability of Results, Research Limitations, and Future

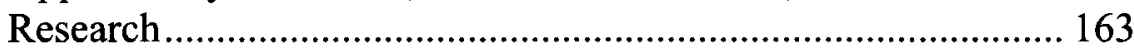

7.0 RESEARCH SUMMARY AND CONCLUSIONS .................................................. 166

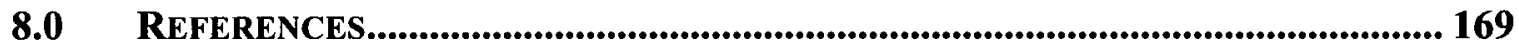

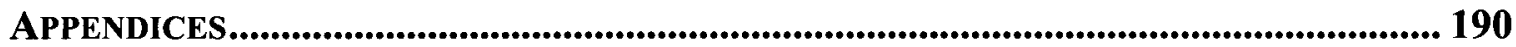




\section{LIST OF TABLES}

Table 1. Summary of field variables measured and derived from field plots representing the various aspects of structural complexity in the forest.

Table 2. Summary of image variables extracted from multispectral airborne imagery to be used as predictors for modelling structural complexity.

Table 3. Final set of field variables collected and calculated within each field plot. Summary statistics shown for each. $(n=50)$

Table 4. Summary statistics calculated from the 500 models (all $\mathrm{p}<$ 0.001 ) that were produced from the first phase of bootstrapping in RDA, which was carried out for variable selection.

Table 5. Results of bootstrapping analysis in terms of sources / types of predictor variables included in the different models (total number of models produced was 500 ).

Table 6. Summary results from selected RDA (produced by CANOCO).

Table 7. Correlation coefficients between field variables and SCI $\left(1^{\text {st }}\right.$ RDA axis) shown in order from strongest to weakest.

Table 8. Biplot scores of RDA1 from final RDA model. Scores show relative importance of each predictor variable towards RDAl and the sign of each shows the relative direction compared to RDA1.

Table 9. Results of field validation for high and low complexity sites, presented following assessment of correct vs. incorrect classification, as well as incorporating partially correct scores of 0.5 .

Table 10. Summary statistics calculated from models produced from the first phase of RDA bootstrapping using predictor variables extracted from $60 \mathrm{~cm}$ and $1 \mathrm{~m}$ imagery independently.

Table 11. Sources / types of predictor variables included in the RDA models produced through bootstrapping the RDA analysis with predictor variables extracted from $20 \mathrm{~cm}, 60 \mathrm{~cm}$, and $1 \mathrm{~m}$ imagery independently. The top three contributors for each resolution are bolded to highlight major differences. 
Table 12. Wood object statistics as derived using the three different classification methods and by intersecting these three preliminary maps into a hybrid classification.

Table 13. Dead wood statistics as measured in the fifty field plots.

Table 14. Regression results for indirect modelling of coarse woody debris volume and the number of snags in fifty plots across the forest. Both models were found to be highly significant $(p<0.001)$. 


\section{LIST OF FIGURES}

Figure 1. Location of study area within the southern section of Gatineau Park near Ottawa, Canada. Shown with 2005 SPOT satellite image in the background. (Courtesy P. Pellikka, University of Helsinki and ESA).

Figure 2. Modified door on Cessna-172 to allow vertical view angle (A) and digital camera setup within the pod of the door (B). Note that a second RGB digital camera is shown mounted in the image, which was being tested at the time.

Figure 3. The final multispectral image mosaic shown within Gatineau Park covering the study area along with the two flight-lines used to acquire the imagery. The fifty field plot locations are also shown.

Figure 4. Net image correction process and results shown for a sample image, with each individual original spectral band shown, along with corresponding correction templates, and the corresponding corrected images. Note that the image looks "flat" following the correction procedure, however this is simply a result of the small scale (i.e. the entire image).

Figure 5. A zoomed in $50 \mathrm{~m} \times 50 \mathrm{~m}$ example of the corrected imagery showing the within and between crown variance, which was still present in the imagery following brightness correction processing. CIR and NIR (greyscale) images are shown.

Figure 6. A subset of the multispectral image mosaic, spanning across the seams of multiple images. Locations of some of the 50 field plots used for the development of a structural complexity index are shown within this area.

Figure 7. Example of field plot setup, with marker at the plot centre and ropes used to mark the diagonals. Understorey and ground vegetation were sampled along these ropes at every $50 \mathrm{~cm}$ (at each orange mark) and coarse woody debris was measured. This was done in order to estimate volume, wherever a piece was crossed by the rope.

Figure 8. RDA modelling process showing two-stage bootstrapping, final model selection, mapping and validation, as well as multiscale model testing. 
Figure 9. Examples of snags thought to be suitable for detecting and mapping, including those with fairly intact crowns (A and B) and those with no branches remaining (C and D), but found in canopy gaps and / or of heights similar to the surrounding live overstorey trees.

Figure 10. Structural conditions typical of area of relatively low structural complexity (A (Plot 2) and B (Plot 35)) and relatively high structural complexity (C (Plot 43) and D (Plot 12)) within the study area.

Figure 11. Example hemispherical photographs taken in a plot with relatively low complexity (Plot 2) (L) as well as one with relatively high complexity (Plot 20) $(\mathrm{H})$. Large differences between the $1 \mathrm{~m}$ and $6 \mathrm{~m}$ photograph can be seen in the high complexity plot as a result of the thick understorey vegetation $(\mathrm{H}-1 \mathrm{~m})$ and the large canopy gaps $(\mathrm{H}-6 \mathrm{~m})$. The opposite was generally true in the low complexity plots, where little understorey vegetation was present to block the camera lens $(\mathrm{L}-1 \mathrm{~m})$ and few significant canopy gaps existed (L-6m).

Figure 12. Triplot for final RDA model. The 1st axis ( $\mathrm{X}$ axis) represents the SCI showing a gradient of structural complexity in the forest. Red vectors represent image and topographic predictor variables $(n=5)$, with the length and direction of the arrow, relative to the $X$ axis, representative of the relative contribution towards the SCI. The blue arrows represent each field variable $(n=24)$ with their length relative to the $X$ axis representative of the amount of explanation provided by that field variable. The smaller the angle between two arrows, the higher the correlation between variables. Note that blue arrows pointing to the left indicate a negative relation with the SCI. The fifty field plots are shown as coloured dots with their position relative to the $\mathrm{X}$ axis representing their modelled level of structural complexity.

Figure 13. Relation between the modeled SCI, shown using a standardized scale from lowest SCI (-2.7) to highest SCI (2.3), and the original qualitative complexity level associated with each field plot through visual assessment. Field plots that showed confusion between classes are circled for each complexity class (i.e. low, medium, high).

Figure 14. Distribution of RMSE (\%) derived through the second bootstrapping phase for selected 5-variable RDA model.

Figure 15. Predicted SCI across the entire study area. The legend for this map, as well as all other SCI maps presented, were created using a standard deviation classification and symbolization. 
Figure 16. Examples of mapped predicted SCI. Masked roads and water bodies are black. (A) Field photo showing low structural complexity, (B) A low complexity area at full CIR image resolution (as outlined in C and D), (C) Subsection of a CIR image showing an area of relatively low complexity (top left box) and an area of relatively high complexity (middle right box), (D) Map of predicted structural complexity corresponding to the image area in C, (E) Field photo showing high structural complexity, and (F) A high complexity area at full CIR image resolution (as outlined in $\mathrm{C}$ and $\mathrm{D}$ ).

Figure 17. An example of partially correct classification of low complexity area. The area $\left(6096 \mathrm{~m}^{2}\right)$ appeared fairly homogenous in the SCI map, however, while the zones labeled A and C were correctly classified as low complexity, the zone labeled B was incorrect, resulting in an overall assessment of a partial correct classification. The field photos labeled "Area A" and "Area B" correspond with the same areas on the SCI map (A was correctly classified as low complexity and B was incorrectly classified as low complexity).

Figure 18. Two sample regions (each $250 \mathrm{~m} \times 250 \mathrm{~m}$ ) extracted from the three different resolution maps of the predicted SCI. The two examples show an area that corresponded well between the different resolutions (Example 1) as well as one that seemed to show significant differences (Example 2) between the lower resolution maps and the original $20 \mathrm{~cm}$ SCI map. Masked water bodies appear as black.

Figure 19. A comparison of the three detection and delineation methods for two large snags shown with the CIR imagery in the background.

Figure 20. An example of the processing carried out to group adjacent wood objects as well as generalize their boundaries. Solid objects shown were grouped and buffered to created objects with boundaries defined by the lines. For visualization purposes only the object-based method is shown as an example, however the same process was carried out for the other two methods as well.

Figure 21. A typical example of detected wood objects shown with the CIR imagery in the background. Spectral unmixing detected many small objects that were not detected by the other two methods.

Figure 22. A $100 \mathrm{~m} \times 100 \mathrm{~m}$ section of the forest shown as an example of the detection / delineation by the three methods as well as the corresponding area of the final map of wood objects derived through the intersection of the three preliminary maps. Both images show the objects with the CIR imagery in the background. 
Figure 23. An example $30 \mathrm{~m} \times 30 \mathrm{~m}$ sample area showing results of testing to determine a suitable threshold for 'hardening' of the unmixed fraction map. Dead wood object numbers and sizes vary with the thresholds shown ( $30 \%$ to $70 \%$ ).

Figure 24. A large dead wood object delineated in the imagery (white outline) that was not seen during field validation. The adjacent and overlapping area of the imagery that is outlined in yellow appears to be a live tree crown seen within a shadow in the canopy.

Figure 25. An example subsection of the map of relative dead wood spatial distribution found across the forest as derived from the CIR imagery, shown for the corresponding area. Roads and water bodies were masked out and appear black on the map.

Figure 26. An example area showing results of a combination of indicators. Areas labeled as A and B were correctly classified as low complexity, however those labeled as $\mathrm{C}$ and $\mathrm{D}$ were found to be misclassified and during field validation were found to contain many snags. In this example, the modification of the original SCI using the dead wood density map produced a more realistic representation of the structural complexity compared to the SCI by itself. 


\section{LIST OF APPENDICES}

Appendix 1: Net Brightness Correction Procedure ................................................. 190

Appendix 2: Bootstrapping Redundancy Analysis (RDA) Procedure........................... 197

Appendix 3: Raw Field Data Including Plot Locations............................................... 199

Appendix 4: Scatterplots showing relationships of field variables with the SCI. line of best fit is included, with plots displayed in an order that corresponds to the correlation coefficients presented in Table 7 . 


\subsection{INTRODUCTION}

The United Nations Convention on Biological Diversity defines biodiversity as "...the variability among living organisms from all sources including, inter alia, terrestrial, marine, and other aquatic ecosystems and the ecological complexes of which they are part; this includes diversity within species, between species, and of ecosystems" (UN-CBD, Article 2, 1993). Thus by definition biodiversity can be examined at three interconnected scales: genetic, species, and ecosystem. At the smallest scale, genetic diversity refers to the diversity of the actual genes that characterize individuals within a species and greater genetic diversity increases a population's chance of surviving changes to their environment as a result of physical differences that provide an advantage to some individuals over the rest of the population (Baydack and Campa, 1999; Smith and Smith, 2001). Diversity at the species level refers to the variety of different species that exist within an ecosystem. Finally, ecosystem diversity refers to the variety of ecosystems in a given place, but also to the variety within ecosystems, in terms of the physical and biological makeup (Smith and Smith, 2001).

Human beings consider biodiversity to be important for a variety of reasons including economic, medicinal, recreational, and spiritual (Ehrlich and Wilson, 1991; Rolston, 1994), and often view biodiversity simply as a means towards an end in terms of providing a product or service (Callicott, 1995). Conversely, many people place intrinsic value on it, valuing it from a biocentric point of view, and placing the value of human beings as equal to that of all other species on the planet (Callicott, 1995; Van de Veer and Pierce, 1998). Regardless of how it is viewed, the conservation of biological diversity is 
essential to humans in terms of the continued use and reliance on natural resources as well as the healthy functioning of ecosystem and sustainability of life on Earth. Ecosystem functions such as carbon sequestration (e.g. Steinbeiss et al., 2008), water purification (e.g. Ostroumov, 2005), and soil formation and health (e.g. Barrios, 2007) are some key examples of such essential functions that are each reliant on the maintenance of biodiversity (Baydack and Campa, 1999; Smith and Smith, 2001). It is also important to keep in mind that while ecosystem functions are dependent on it, the compositional diversity of an ecosystem itself is tied directly to the structural and functional diversity within that ecosystem. The structure of the landscape, in terms of the physical organization or patterns, can have an impact on species diversity in terms of the physical habitats that exist. As well ecosystem functions such as disturbances can have significant impacts on compositional diversity, both directly, and indirectly by altering the structure of the landscape (Franklin et al., 1981; Noss, 1990).

With growing concerns over the loss of biodiversity, the United Nations Convention on Biological Diversity (CBD) was initiated in 1992 and currently involves one-hundred and ninety-one countries around the world. The CBD provides a framework of policies and goals, but not hard targets and obligations, which focus on the conservation of Earth's biodiversity and sustainable use of biological resources (Glowka et al., 1994). Within the CBD, countries are attempting to make "...a significant reduction of the current rate of biodiversity loss at the global, regional and national levels" (CBD COP 6 Decision VI/26) by 2010. 
While generally speaking the CBD deals with global biodiversity conservation, of particular interest to this research is the CBD's adoption of an expanded programme of work on forest biological diversity (CBD COP 6 Decision $\mathrm{VI} / 22$ ). Within this programme, the CBD supports the development of methods for monitoring forest biodiversity, and specifically for the development and implementation of indicators, which allow researchers to compare biodiversity levels over time to some baseline condition (Noss, 1990; Noss, 1999; Niemela, 2000).

The Canadian government ratified the CBD on December $4^{\text {th }}, 1992$ and following this, the Canadian Biodiversity Strategy was developed. This strategy outlined how Canada would create the necessary policies and research conditions in order to achieve the goals set by the CBD (Biodiversity Working Group, 1994). Focusing on ecological management capabilities, within this document the Canadian government clearly stated its interest, and need, to:

"Focus or enhance research to increase our understanding of ecosystems and our ability to manage human use of ecosystems and resources, by developing cost-effective biodiversity inventory and monitoring methods and programs, including rapid assessment procedures and biodiversity indicators, to detect and monitor changes to ecosystems, species and genetic diversity" (Biodiversity Working Group, 1994). 
The research presented in this thesis integrated aspects of both geomatics and conservation biology, with the focal point being the measurement and modelling of forest structural conditions. This followed the need for the development of biodiversity indicators, specifically spatially extensive ones, which are repeatable in nature allowing long-term monitoring of forest biodiversity. Specifically at the local scale, long term sustainable biodiversity and forest management can greatly benefit from methods for modelling the spatial distribution of available habitat and biodiversity levels across a forest as well as monitoring changes within the forest over time.

Conservation involving the identification of sites with potentially high biodiversity value is important both for comparing a forest of interest to other similar ones, or at a finer scale within a single forest (Noss, 1999; Smith et al., 2008). However, it is also important to locate areas with potentially low biodiversity where site remediation to increase biodiversity levels could possibly be incorporated into management plans (e.g. the creation of artificial snags (Chambers et al., 2002; Pollard and Moi, 2006)).

Comprehensive field-based species surveys and habitat sampling cannot provide spatially extensive or continuous information across a forest. More importantly, this type of field work requires highly trained individuals, and can be very costly in terms of time and resources, making such inventories very difficult especially across large forests (Lawton et al., 1998; Ricketts et al., 1999; Rainio and Niemela, 2003). Remotely-sensed imagery can provide spatially continuous information over large areas and has been used successfully in the past for measuring and monitoring different aspects of forests for use 
in forest inventories (e.g. Wulder, 1998; Lefsky et al., 2001), biomass / carbon budget modelling (e.g. Luther et al., 2006; Blackard et al., 2008), as well as the assessment and mapping of disturbance and damage (e.g. Cosmopoulos and King, 2004; Olthof et al., 2004; Wulder et al., 2008). Additionally, the repeatable nature of remote sensing acquisitions makes it an ideal source of information to be included in monitoring programs.

Through theory as well as field studies, research has shown a connection between the structural complexity of a forest, referring to the abundance and variability of horizontal and vertical structural attributes present (McElhinny et al., 2005), and increased biodiversity of both flora and fauna through the increased amount and variety of available habitats available in an ecosystem (e.g. MacArthur and MacArthur, 1961; Newsome and Catling, 1979; August, 1983; Freemark and Merriam, 1986; Williams et al., 2002; Lassau et al., 2005). As well, in terms of a specific aspect of forest structure, dead wood in a forest is considered essential for providing habitat and supporting diversity of birds, invertebrates, and small mammals (e.g. McComb and Lindenmayer, 1999; Arsenault, 2002; Bull, 2002).

Remote sensing has been used extensively for modelling and mapping a variety of individual forest structural attributes, including Leaf Area Index (LAI), canopy closure, basal area, tree heights and dead trees (for a selection of examples see Hall et al., 1995; Franklin et al., 1997; Wulder, 1998; Lim et al., 2003; Pu et al., 2003; Kelly et al., 2004; Leckie et al., 2004; Hudak et al., 2006; Guo et al., 2007; Breidenbach et al., 2008; Vega and St.-Onge, 2008). On the other hand, research and practical applications that involve 
modelling of the multivariate aspects of forest structural complexity, in terms of the interaction of multiple attributes, is comparatively limited (Coops and Catling, 1997a and 1997b; Coops et al., 1998; Sampson et al., 2001; Cosmopoulos and King, 2004; Wunderle et al., 2007; de la Cueva, 2008). This is particularly true for research pertaining to hardwood forests (Sampson et al., 2001). As well, as a result of the limited research that has focused on mapping dead trees (Haara and Nevalained, 2002; Butler and Schlaepfer, 2004; Kelly et al., 2004; Leckie et al., 2004; Guo et al., 2007; Meentemeyer et al., 2008), the potential use of high resolution remote sensing for mapping dead tree spatial distribution could be explored further, particularly in a temperate hardwood forest.

\subsection{Research Contributions}

This thesis presents new methods for modelling and mapping structurally-based indicators, or indirect measures, of biodiversity within a temperate hardwood forest. In doing so, it contributes to ongoing research involving the use of geomatics, and more specifically remote sensing, to map forest structural conditions. This research used high resolution multispectral airborne imagery, which permitted the exploration of the extensive, and often ignored, spatial information inherent in imagery of forests, in addition to detailed spectral information. This information was used to investigate structurally-based indicators that have been previously associated with high levels of biodiversity in forests: (1) modelling and mapping forest structural complexity, and (2) the detection and mapping of the spatial distribution of dead wood.

With respect to the use of high resolution imagery for semi-automatic mapping of dead wood, indirect modelling and direct detection methods were examined. This 
research was unique in terms of the predictor variables used for indirectly modelling dead wood, and in terms of the hybrid classification technique used for the direct detection. As a unique aspect of this research, the detection of dead trees and individual branches was explored, rather than simply detecting and mapping dying tree crowns. Finally, this research attempted to map the spatial distribution of dead wood within a temperate hardwood environment as an indicator on its own, and to be used in combination with other indicators, including a map of structural complexity.

With respect to overall structural complexity as an indicator, this research used a robust Redundancy Analysis (RDA), which is a statistical technique yet to be fully explored and exploited by the remote sensing community. Structural complexity, as represented by a comprehensive set of field variables, was modeled using empirical models derived through RDA. An extensive set of image variables, including spectral, spatial, and object-based information derived from the imagery was used as predictor variables in order to test the ability for modelling plot-based structural complexity. These variables were also used to explore the types of information that were most useful for explaining structural complexity. In addition to standard spectral and spatial variables, this analysis used information from within-crowns as well as within-shadows in the canopy, which were hypothesized to potentially be more useful than plot-level image information in relating the imagery to conditions found in the field.

Perhaps one of the most novel areas of research that was investigated was the utilization of RDA directly for spatially continuous predictive mapping of structural complexity. The bootstrapping process, as well as field validation of the RDA modelling 
and mapping itself, were unique relative to the existing literature in terms of their capacity to sufficiently and independently validate the process and the resultant models and maps.

Finally, keeping in mind the somewhat limited use of high resolution airborne imagery over large areas, this research explored the potential for scaling the modelling and mapping methods up to coarser scales by testing the methods using simulated imagery of different spatial resolutions.

\subsection{Thesis Structure}

This thesis focuses on the development of geomatics based methods for mapping two potential indicators of habitat and biodiversity within a temperate hardwood forest: (1) forest structural complexity, and (2) the spatial distribution of dead wood across the forest. Chapter 3 contains extensive background information drawn from the pertinent literature. This includes detailed reviews of the relation between biodiversity and structural complexity, biodiversity modelling and mapping using remote sensing, as well as modelling and mapping forest structural attributes using remote sensing. Chapter 4 details the methods that were carried out for the various analyses included in this research. Chapter 5 presents the results of structural complexity modelling and mapping as well as the indirect and direct detection and mapping of dead wood. Chapter 6 provides a complete discussion of the results of the various analyses, and a discussion of the limitations, potential uses of the results, and future research potential based on the results presented in this thesis. Finally, Chapter 7 presents a summary and conclusion of the research. 


\subsection{Research OBJECTIVES}

The following research objectives were arrived at following a review of the current literature on forest structural modelling involving remote sensing and an evaluation of existing research needs within the context of developing spatially continuous and extensive indicators of habitat and biodiversity.

1) Develop a geomatics-based method using high resolution multispectral airborne remote sensing and topographic information for modeling multivariate structural complexity within a temperate hardwood forest;

2) Map predicted structural complexity continuously across the study area;

3) Validate the map of predicted structural complexity using independent field information;

4) Explore the potential for modeling and mapping structural complexity at coarser scales using simulated lower resolution imagery;

5) Determine the potential of high resolution multispectral remote sensing to indirectly model and directly detect the presence of dead wood in a temperate hardwood forest, including independent field validation;

6) Map the spatial distribution of dead wood across the study area;

7) Investigate the potential for combining multiple indicators. 


\subsection{BACKGROUND}

\subsection{Biological Indicators}

Instead of carrying out intensive species surveys, which is impractical given time and resource constraints (Hammond, 1994; Ricketts et al., 1999; Rainio and Niemela, 2003), surrogate measures, commonly known as biological indicators or bioindicators, have been developed in order to provide indirect measures of biodiversity levels. For many years, bioindicators have been used to provide measures of the changing environment. Some examples of bioindicator applications include indicating the presence of environmental pollutants or contaminants (e.g. Cairns, 1979; Onianwa, 2001; He et al., 2005; Pesch and Schroeder, 2006), climate change (e.g. Buntgen et al., 2005; KarstRiddoch et al., 2005; Przybylak et al., 2005; Ruhland and Smol, 2005; Smol et al., 2005), as well as the general health of ecosystems (e.g. Shear at al., 2003; Grey and Azuma, 2005).

\subsubsection{Species-Based Indicators of Biodiversity}

A single indicator species, or a suite of indicator species, have often been used as indicators of the presence or absence of other species, as well as for indicating the overall health of the ecosystem (Caro and O'Doherty, 1999). An indicator species can be defined as a species whose characteristics (i.e. presence or absence, population density, dispersion, reproductive success) can be used as an index for attributes too difficult, inconvenient, or expensive to measure for other species or environmental conditions of interest (Landres et al., 1988). Specifically related to biodiversity, their richness and 
diversity can reflect the diversity of other species in the ecosystem (Rainio and Niemela, 2003). Biologists have often used the concept of keystone species, which refers to a species whose presence, more so than other species in an ecosystem, is crucial in maintaining the organization and diversity of its ecological community (Mills et al., 1993). While keystone species are often used as indicator species, they can be difficult to identify, and not all ecosystems necessarily have one (Beazley and Cardinal, 2004).

Several factors must be considered when selecting indicator species. They should be taxonomically well-known and stable and the biology and general life history (i.e. limiting resources, physical tolerance, etc) must be well understood (Pearson, 1994). Perhaps most importantly, they must be particularly sensitive to whatever is being studied (Landres et al., 1988). Lastly, if the species is to be monitored it is essential that it can be easily surveyed (Pearson, 1994). Landres et al. (1988) noted that while large species are usually much easier to survey and have potentially more stable populations, smaller species are much better choice because they have shorter generations allowing changes to be seen much faster.

Selecting an indicator species for monitoring biodiversity is not straightforward in complex ecosystems, and often a complementary set of indicators is required (Noss, 1990). Beazley and Cardinal (2004) described a systematic approach for selecting species for conserving forests in Nova Scotia, Canada involving large matrices incorporating thirty-three characteristics. The characteristics were grouped by rarity / population status (i.e. is it rare? is the population declining?), biological traits (i.e. space demands? susceptible to pollution?), habitat-related vulnerability (i.e. climatic sensitivities?), 
human-impact factors (i.e. legally harvestable? habitat threatened by loss?), and information status (i.e. is distribution monitored?). They constructed matrices for fortyseven mammals, nine reptiles, thirteen amphibians, and thirty-three freshwater fish that were native to Nova Scotia. Expert biologists were surveyed and using 'yes', 'no', 'maybe', or 'unknown' as possible responses, species were selected that were representative of the different characteristics. Based on the compiled results several species were identified as potential indicators.

The conservation biology and ecological literature is filled with different uses of indicator species as surrogate measures of biodiversity. For example, because of the great abundance, diversity, functional importance, sensitivity to disturbances, and the ease with which they can be sampled, invertebrates have proved to be useful indicators. This is perhaps best captured by Andersen et al. (2004), who, referencing other researchers, stated that "no biodiversity monitoring program can be considered credible without the inclusion of invertebrates". Beetles specifically are widely used invertebrate indicators of forest biodiversity (e.g. Rainio and Niemela, 2003; Langor and Spence, 2006), and more specifically Carabid beetles, or ground beetles. These beetles are incredibly diverse and each species, or assemblage, live in certain habitats based on temperature and humidity, available food, and the presence and distribution of competitors (Rainio and Niemela, 2003). As well, like most invertebrates, beetles are easily surveyed, and as a result of the fast turnover rate and large sample size available, one can expect them to respond rapidly to disturbances over short time periods (Pearce and Venier, 2006). 
The potential of birds as biodiversity indicators is based on similar concepts to those seen for invertebrates. The high species diversity exhibited by birds allows for monitoring of a variety of underlying ecosystem processes (Venier and Pearce, 2004). For example, Mikusinski et al. (2001) used woodpecker species as indicators of bird diversity in European forests. They based their theory on the fact that woodpeckers were considered to be "a demanding group among European forest birds", and as a result, the presence of several species of woodpeckers indicated certain properties about the forest. The authors compared woodpecker species richness with the species richness of other forest birds and found significant positive correlations. In fact the presence of the most specialized woodpecker species was actually the best predictor of forest bird diversity. Further, Drever et al. (2008) showed woodpecker richness as a useful indicator of overall bird richness in mixed forests in British Columbia, Canada.

Ricketts et al. (1999) carried out an analysis across the ecoregions of North America, looking at pairwise comparisons and overall comparisons of amphibians, birds, butterflies, mammals, reptiles, land snails, trees, vascular plants and tiger beetles, using both richness values in each ecoregion as well as overall richness indices. Their analysis showed that all pairwise correlations were significant, however, mammals had the weakest correlations with other groups. The results were somewhat diminished when they took into account latitudinal effects across North America, which appeared to be responsible for a large amount of variation in species richness. 


\subsubsection{Structural Indicators of Biodiversity}

While perhaps not as widely used, landscape spatial and structural information has also been used for biodiversity monitoring. Noss (1990) developed an indicator selection matrix that provides measurable indicators for overall biodiversity. This matrix, based on Franklin et al. (1981)'s discussion of three general ecosystem attributes for describing indicators of overall biodiversity, included four scales ranging from genetic to landscape. More than simply looking at an ecosystem's composition (i.e. species diversity), he proposed including measures of an ecosystem's structure, referring to the "physical organization or pattern of the system", as well as monitoring the functional aspects of an ecosystem, including disturbances and nutrient cycling. In addition to including commonly used indicators such as species distribution, richness, diversity, which correspond with the indicator species concept, Noss presented many indicators that relied on measures of structure at the landscape and ecosystem level representing physical attributes of the system.

Spatial measures such as the patchiness, configuration, and fragmentation of the landscape, as well as more basic measures such as patch size, perimeter-area ratio, and patch size frequency distribution, can all potentially be used as indicators for biodiversity. This is possible as a result of the link between landscape heterogeneity and species diversity (Noss, 1990; Atauri and de Lucio, 2001; Opdam et al., 2003). Issues of connectivity (i.e. corridors between patches) and heterogeneity (i.e. patch arrangement) are thought to be very important for the existence of viable populations and healthy ecosystems (Lindenmayer et al., 2000). A wide variety of landscape composition and 
configuration measures exist, ranging from simple to very complex. The key benefits of these measures is their complete independence from species (i.e. field) data and the relatively simple and inexpensive methods needed to calculate them given a landcover map of the appropriate scale (Opdam et al., 2003).

\subsubsection{Keystone Structures Including Dead Wood}

At the local scale, Tews et al. (2004) presented the concept of keystone structures as attributes in an ecosystem that provide resources, shelter or 'goods and services', which are crucial for the survival of species within the ecosystem. These structures are vital to maintaining biodiversity levels and their removal would likely lead to a breakdown in species diversity since so many species rely on them (Tews et al., 2004). In a forest ecosystem, standing and downed dead wood can be considered excellent examples of keystone structures as they are widely accepted to be essential for the sustainability of biodiversity (McComb and Lindenmayer, 1999; Arsenault, 2002; Bull, 2002).

Historically, forest dead wood was not considered to be useful and in fact, it presented a danger to forest firefighters by providing increased fuel in the forest, and as well standing dead trees had the potential to become unstable and fall (Thomas, 2002; Stephens and Moghaddas, 2005). However, by the 1970s, foresters and researchers became more aware of the importance of dead wood in forest ecology (Arsenault, 2002; Thomas, 2002), and since then dead wood has been researched in great detail with respect to its ecological importance in forests. 
A wide variety of mammals, birds, amphibians, reptiles, and invertebrates make use of standing dead trees, commonly referred to as snags, mostly using cavities within the dead trees (McComb and Lindenmayer, 1999). While many more species of invertebrates than vertebrates directly use snags, invertebrates, as part of the food chain, represent a food source for the higher up vertebrate species (McComb and Lindenmayer, 1999; Bull, 2002). Birds are probably the most abundant vertebrate that use snags, with up to $40 \%$ of species in North American forests classified as cavity nesters (McComb and Lindenmayer, 1999). Some species, for example woodpeckers, are considered primary cavity nesters, while other species, known as secondary nesters, make use of existing and vacated cavities as well as hollows inside tree trunks (Stribling, 1990; Bull, 2002; Hartwig et al., 2002; Drapeau et al., 2003; Spiering and Knight, 2005). Many other species also make use of cavities, for example red and flying squirrels, martens, fishers, porcupines, bobcats, as well as black bears (Griesemer et al., 1998; Taulman, 1999; Bull, 2002; Zielinski et al., 2006). In general, snags or dead limbs $<10 \mathrm{~cm}$ in diameter are not considered to have very much value for nesting vertebrate, although invertebrates, such as ants and beetles, and fungi, will still exploit the dying wood (McComb and Lindemayer, 1999). As well, when a living tree is wounded, parts of the tree may actually function as a snag and provide habitat to a variety of species (Bull, 2002).

Woody debris includes logs, coarse roots and stumps at all stages of decay. Specifically, coarse woody debris (CWD) is considered to be woody debris with a diameter greater than $7.5 \mathrm{~cm}$ (Stevens, 1997; CFIC, 2004). CWD originates from fallen branches, tops of trees that have broken off, entire dead trees that have fallen and through logging activities. While natural death is certainly a source of woody debris, strong 
winds, fires, insect damage, disease, slope failure, and flooding are common causes for dead or dying wood to end up on the ground (Stevens, 1997; Bull, 2002).

CWD increases the composition and structural complexity of the forest floor and provides a variety of habitats, for example, acting as a seedbed or nursery site for mosses, ferns, shrubs, and regenerating trees (McComb and Lindenmayer, 1999; Takahashi et al., 2000). It is also critical for ecosystem function, adding a significant amount of organic matter to the soil, contributing to soil acidification and podzolization, albeit certainly not in the short term (Stevens, 1997; Stephens and Moghaddas, 2005). As well CWD can help prevent soil slumping by providing some stabilization on slopes (Stevens, 1997; Stephens and Moghaddas, 2005). Many vertebrates and invertebrates use the debris for a for den, nest, and foraging sites, hiding places from predators, and refuge spots during disturbances (Stevens, 1997; McComb and Lindenmayer, 1999; Hammond et al., 2001; Arsenault, 2002; Bull, 2002; Payer and Harrison, 2003; Ulyshen et al., 2004; McKenny et al., 2006). From a forest management perspective, in order to maintain biodiversity dead wood must be included in management strategies along with live trees (Franklin et al., 1981).

\subsubsection{Habitat Heterogeneity Hypothesis}

These concepts of structural heterogeneity within an ecosystem have been tied together by a general hypothesis. The habitat heterogeneity hypothesis reflects relations between ecosystem structure and biodiversity levels at multiple scales, including within individual forests, which is of most interest in this thesis. More complex areas should exhibit greater biodiversity as a direct result of the greater number and variety of habitats 
available which species can use to exploit the environment, compared to structurally simpler areas (Klopfer and MacArthur, 1960; MacArthur and MacArthur, 1961; Bazzaz, 1975; Freemark and Merriam, 1986; Zenner, 2004; McElhinny et al., 2005). While a variety of terms have been used in the literature including habitat heterogeneity, structural heterogeneity, structural diversity, habitat complexity, and habitat diversity, this thesis focuses on "structural complexity", used to refer to the abundance and variability of horizontal and vertical structural attributes present in the forest (McElhinny et al., 2005).

Tews et al. (2004) reviewed studies investigating habitat heterogeneity and species diversity and found some studies did not support this hypothesis, (e.g. for small mammals (Sullivan and Sullivan, 2001), birds (Ralph, 1985), and butterflies (Hill et al., 1995)). However, most studies, particularly those investigating beetles, birds, and small mammals specifically in forest ecosystems demonstrated this relationship (e.g., MacArthur and MacArthur, 1961; Newsome and Catling, 1979; August, 1983; Williams et al., 2002; Lassau et al., 2005). Tews et al. (2004) pointed out that discrepancies found by some researchers were often a result of how diversity was measured, how heterogeneity was defined, as well as the temporal and spatial scale of the study. At some scales increased heterogeneity can in fact cause a reduction in species diversity, for example landscape fragmentation can disrupt processes such as dispersal and resource acquisition.

The habitat heterogeneity hypothesis originates from the early work of MacArthur and MacArthur (1961) who noted that it was common knowledge that more species of 
birds were found in a forest than a field, but additionally that more species were found in tropical forests compared to temperate ones. Based on these observations, they examined bird diversity in predominantly deciduous forest plots across the United States and measured vertical profiles of vegetation as a measure of foliage height diversity. Their findings showed a significant positive linear relation between bird diversity and foliage height diversity, and discussed the vertical layers as being patches, similar to landscape patches, which the birds moved between and utilized for different purposes.

August (1983) showed a strong positive curvilinear relationship between vegetation complexity (as measured by a cumulative structural complexity measure that incorporated various attributes including canopy height, shrub density, canopy cover, and tree sizes) and small mammal diversity in tropical rain forest in Venezuela. Lassau et al. (2005), working in Australian forests, investigated beetle diversity in comparison to habitat complexity, measured by a cumulative score based on tree canopy cover, shrub cover, herb layer cover, soil moisture, amount of leaf litter, and debris (logs and rocks). Their findings showed habitat complexity to be a strong predictor of beetle richness and abundance for beetles caught on the ground, but not those caught using flight intercept traps. Further analysis of individual structural attributes led them to the conclusion that it was unlikely that a single habitat characteristic would control beetle diversity, and as a result a composite score was needed.

\subsubsection{Structural Complexity Indices}

The development of indices of structural complexity has been a topic investigated by forest researchers for more than thirty years. McElhinny et al. (2005) defined 
structural complexity indices as mathematical constructs that summarize two or more structural attributes in a single number or index value. Catling and Burt (1995) suggested a need for descriptors derived from forest structural attributes that could provide local descriptions of habitat, allowing the prediction of distribution and abundance of wildlife. However, as previously mentioned, it is understood that more than one, and often many, structural attributes in a forest are important for controlling species diversity and therefore need to be incorporated into any structurally-based assessment related to biodiversity levels.

Indices have been derived through a variety of approaches, with several examples presented here to provide an idea of the range of techniques used. Perhaps the simplest method involves scoring of individual attributes and the summing of these scores to produce a single index value. Newsome and Catling (1979) established a method of scoring a forested area based on five structural attributes that were each given a score ranging from zero to three. Their habitat complexity score, derived through visual scoring carried out at field sites, incorporated canopy cover, shrub cover, ground vegetation cover, soil moisture, and debris, including logs and rocks. This index has been used in research involving small mammal diversity and habitat (Newsome and Catling, 1979; Catling and Burt, 1995) as well as investigating habitat complexity related to time since fires in Australian forests (Coops and Catling, 2000). The index was also used by Lassau et al. (2005), as discussed in the previous section, with an additional parameter describing the amount of litter to describe beetle diversity. 
Van den Meersschaut and Vandekerkhove (1998) presented a structural complexity index developed in order to help evaluate the overall biodiversity potential of forests. Their index, based on eighteen structural attributes, included structural information related to canopy closure, vertical layering of the canopy, the number of large trees, variation in tree size, understorey layer information, as well as various measurements quantifying snags and woody debris. Unlike the scoring system developed by Newsome and Catling (1979), which used broad thresholds to derive scores for different attributes, this index involved quantitative measurements of each attribute (i.e. calculating the actual basal area of all snags or counting the number of logs) in the plots and a scoring system with detailed instructions as to how to derive partial scores for each attribute. Different attributes were then provided with different weightings and the scores were summed, with a maximum biodiversity index score of one-hundred.

Other indices have been created, which, according to McElhinny et al. (2005), are based on the interaction of different structural attributes. Indices described by Staudhammer and LeMay (2001) as well as Zenner (2000) involved mathematical calculations of complexity based on actual field measurements of individual tree diameter and height, without any arbitrary weightings and scoring. Staudhammer and LeMay's (2001) stand variance index (STVI) was developed as a monitoring index to indicate vertical and horizontal changes in association with forest growth and yield modelling. This STVI was later extended by LeMay and Staudhammer (2005) to include spatial distribution as a measure of structural diversity through the inclusion of an inter-tree distance calculation. 
Tree distribution information has been of interest to researchers for many years and has been the basis of several simple indices, which are generally used to describe the regular or clumped distribution of trees. For example the Clark-Evans Index (Clark and Evans, 1954) and the Pielou Index of non-randomness (Pielou, 1959), simply require plot stem density and inter-tree distance measurements and can be used to differentiate between areas of the forests in terms of their horizontal complexity.

More advanced indices relying on tree distribution have been developed. For example, Zenner (2000) and Zenner and Hibbs (2000) presented a structural complexity index, which expressed the variability of tree diameter or height with respect to inter-tree distances in order to compare variability between forest stands. By calculating a threedimensional triangulated irregular network (TIN) using each tree's location ( $\mathrm{x}$ and $\mathrm{y}$ coordinate) and either the diameter or height as the $\mathrm{z}$ coordinate, the index was able to differentiate between simple and complex stands by examining the number and size of triangles within each plot.

Statistical combinations of structural attributes have also been used in order to describe the structural complexity in a forest. For example, the research of August (1983) used the first principal component from a PCA derived from field measurements, which incorporated structural attributes including canopy height and density, foliage height diversity, as well as the number and size of trees. He differentiated complex habitats from simple ones in terms of the vegetation strata and foliage density, with complex habitats having more strata and more dense foliage at all levels. Combining these various attributes into a single index value, represented by the first principal component, allowed 
August to use complexity as a predictor of mammal diversity. Van Pelt and Nadkarni (2004) also used a PCA to reduce the dimensionality of their thirteen-variable dataset representing different attributes of Douglas Fir forest stands in southern Washington, U.S.A. Their first component, which represented $68.5 \%$ of the variance, was used to rank stands in terms of their complexity using only a single variable, which in their case was found to be linearly related with stand age.

McElhinny et al. (2006) presented further complexity index research, which was created for differentiating vegetation types as opposed to assessing biodiversity. They presented an objective and quantitative methodology in order to construct an index that represented stand structural complexity. by: (1) establishing a comprehensive suite of stand structural attributes, (2) quantifying the different attributes by collecting field data in the vegetation communities in which the index is intended to operate, (3) identifying a core set of attributes through analysis of the collected data, and finally (4) combining the core attributes in a simple additive index. The attributes are then scored relatively based on their observed levels since structural complexity is a relative rather than absolute concept, as opposed to using arbitrary weightings.

McElhinny et al. (2006), following an extensive review of existing indices (McElhinny et al., 2005), established a comprehensive suite of structural attributes that included measures of canopy cover, stand height, tree dbh, dead trees, stumps, understorey cover, CWD, litter, and ground cover. They systematically reduced the number of variables following the collection of field data in order to remove redundant measures. For example, they found stand basal area to be correlated with canopy cover $(\mathrm{r}$ 
$=0.77)$ and therefore used only stand basal area due to the fact that it was an easier and more precise variable to measure. In addition to removing redundant variables, they also focused on those attributes that showed significant differences between two types of forests in question. In order to avoid arbitrarily scoring the different attributes, they rescaled each variable to scores from zero to ten by regressing the data against quartile midpoints. This provided equal weights to each attribute, but permitted them to be combined by adding them together to create an index value ranging from zero to onehundred and thirty.

\subsection{Modelling and Mapping Biodiversity and Biodiversity Indicators with Remote Sensing}

While the biodiversity indicators that have been presented up to this point, including structural complexity indices, are strictly field-based, remote sensing has been used in a variety of ways to indirectly model and map biodiversity levels and to develop forest structural complexity indices based on associations between remotely-sensed imagery and forest structural attributes.

\subsubsection{Indirect Modelling and Mapping of Species Distribution and Biodiversity}

Remote sensing can provide tools for indirectly modelling and mapping biodiversity, and therefore allow for long term repeatable monitoring. It can be used to map habitat preferences of species through the use of thematic mapping, as well as monitoring ecosystem functions, which themselves can be linked to biodiversity levels. 
Nagendra (2001) discussed the use of remote sensing for analyzing species distribution patterns, and presented three main categories: (1) those that directly mapped individual plant species, usually in large contiguous areas, (2) those that mapped habitat in order to predict species distribution based on habitat requirements, and (3) those that modeled the relationships and patterns between species distribution and remotely-sensed data. The first group of studies involved research that ranged from delineating individual tree crowns and classifying their species using airborne imagery to using Landsat imagery for mapping homogeneous stands.

The second use of remote sensing relies on knowledge of habitat preferences of individual species, and by creating land cover maps using various classification techniques, the critical habitat can be isolated. For example, Bechtel et al. (2004) investigated links between Woodland Caribou presence in northern Alberta, Canada and spectral clusters derived from Landsat TM imagery, which were assigned to land cover types (e.g. black spruce, shrubby fen, and open water) based on visual interpretation. This analysis resulted in methods to map habitat over very large areas in short periods of time through the simple associations derived from caribou presence information and land cover information. Similarly, but involving more than a single species, Debinski et al. (1999) used clustered Landsat TM data covering Yellowtone National Park, U.S.A. to examine relations between land covers and species of birds, plants, and butterflies. Their results showed significant relations between many of the species and associated land cover types, including rare butterfly species with highly specialized habitats. 
Land cover maps derived from remotely-sensed imagery have also been used to calculate landscape metrics, or structural measurements of landscapes, which can be related to biodiversity distribution. For example, Kerr et al. (2001) derived a land cover diversity metric by calculating the number of different land cover types derived from a national scale SPOT VGT (1 km x $1 \mathrm{~km}$ pixels) land cover classification found within quadrats from grids spread across Canada. They found land cover diversity to be a very strong predictor of butterfly richness, as calculated from 113525 records for 297 butterfly species across Canada.

Within Nagendra's (2001) second category, local species-based mapping can be carried out if specific habitat requirements for a species are known, which can be linked to remotely-sensed information. Pasher et al. (2007) provided an example of this as they used Ikonos (4 m x $4 \mathrm{~m}$ pixels) to predict and map suitable habitat for Hooded Warblers in southern Ontario, Canada by indirectly mapping canopy gap characteristics, which were shown in the field to be related to nest site selection.

The final group defined by Nagendra (2001) involves indirectly relating spectral characteristics from imagery to species distribution, based on ecosystem function, this time with no direct link. The Normalized Difference Vegetation Index (NDVI), which is easily calculated from remotely-sensed imagery at a variety of scales, is known to provide a good measure of photosynthetically active vegetation and has been shown to be correlated with climatic variables such as rainfall, temperature, and evapotranspiration, providing useful information on productivity (Oindo et al., 2000; Oindo and Skidmore, 2002; Skidmore et al., 2003; Olthof and Latifovic, 2007). These variables, related to heat 
and energy aspects of the climate, are considered very important predictors of species distribution at small scales, especially in cold or temperate terrestrial regions such as Canada (Kerr et al., 2001).

Oindo et al.'s (2000) research attempted to link ten years of NDVI data with bird species richness in Kenya, and then map diversity across the country. They expected higher NDVI to represent high productivity and support a greater number of bird species. These expectations were premised on the fact that birds used the vegetation for food, perches, nesting, and breeding. They found a strong positive correlation $(r=0.71, p<$ 0.05 ) between species diversity and the average NDVI within the sampling regions, which was calculated by averaging peak growing season NDVI (i.e. maximum) values for each pixel within the region. Using this relation and linear regression modeling, they were able to map bird species richness. This approach was largely successful aside from the poor accuracy seen in large lakes, which had very low NDVI values but did contain many bird species. As well, errors were seen in the small very humid areas in northern Kenya that had higher predicted richness than actually existed.

Oindo and Skidmore (2002) built on their earlier work in examining mammal and plant distribution across Kenya using NDVI data, and then later examining mammal species richness in Kenya using NDVI compared to temperature and potential evapotranspiration (Skidmore et al., 2003). Interestingly, results from their research showed NDVI to have a parabolic relationship with species richness. The highest richness was found at the top of the parabola, representing intermediate productivity, which they found to be consistent with previous studies, albeit possibly a result that was 
dependent on the specific scale of study, and potentially not valid at other scales, specifically more local ones. Seto et al. (2004) carried out a similar experiment examining Landsat derived NDVI data as a predictor for bird and butterfly distribution across the Great Basin of North America. They tested the hypothesis that species distribution was dependent on primary productivity (mean and maximum NDVI), but also incorporated a measure of vegetation heterogeneity (standard deviation of NDVI). Their results showed that while at the regional scale (i.e. within each of the three regions in their landscape) relationships were weak, an analysis of the entire landscape showed all measures of NDVI to be related to species richness using linear and quadratic relations. Their multiscale analysis stressed the importance of carrying out such analyses at scales appropriately selected to represent the specific application.

Phillips et al. (2008) explored the predictive potential of NDVI for explaining bird richness across North America, as well as estimates of gross primary production (GPP) and net primary production (NPP), which are considered to be more direct measures of ecosystem productivity. The three variables were derived from satellite imagery reflectance (MODIS $1 \mathrm{~km} \times 1 \mathrm{~km}$ data) as well as, when needed, land cover maps and modeled climate variables for estimating vegetation growth. Bird richness data was taken from species count data collected along bird survey routes, which had been collected in the years that coincided with the MODIS data (2000-2004). Using all of the data, they found all three representations of productivity to be strongly correlated with bird species richness (correlations ranged from 0.50 to $0.52(\mathrm{p}<0.001)$ ), with the three predictors strongly correlated with one another as expected (correlations ranged from 0.91 to 0.95 (p $<0.001)$ ). Once the vegetation classes were stratified, the benefits of GPP and NPP were 
seen, with stronger relations between productivity and richness found in areas of sparse (i.e. dominated by bare ground) as well as dense vegetation compared to relations using NDVI.

While productivity measures have proven themselves useful for modelling and mapping biodiversity at regional and national scales, local scale habitat and biodiversity monitoring using remote sensing has not been explored to the same degree. Having already discussed the utility of field-based structural complexity indices as well as other structural indicators for measuring and monitoring forest biodiversity, and given the wellestablished relations between remotely-sensed imagery and forest structure, the possibility exists for using a remote sensing based approach for modelling, mapping, and eventually monitoring changes in a forest's structural complexity, which could possibly contribute to modelling and monitoring local habitat and biodiversity changes in the forest.

\subsubsection{Modelling Forest Structure with High Resolution Remote Sensing}

A variety of individual forest structural attributes, both horizontal and vertical, have been successfully modeled using remotely-sensed imagery. Specifically, modeling of leaf area index (LAI), canopy closure, crown diameter, basal area, stem density, and tree height have produced significant relations using high-resolution multispectral airborne imagery and satellite imagery. While a variety of sensors have been used to model forest structural attributes, including airborne synthetic aperture radar (SAR) (Kellndorfer et al., 2001; Nelson et al., 2007; Breidenbach et al., 2008) and lidar (Lefsky et al., 2002; Lim et al., 2003; Hyde et al., 2005; Hudak et al., 2006; Breidenbach et al., 
2008), this research focused on the use of high resolution airborne multispectral imagery. This approach currently presents advantages of generally lower acquisition and processing costs for small coverage, greater availability in terms of the number of airborne digital cameras in commercial operation and more straightforward interpretation that is based on a long tradition of photographic imagery analysis. However, it is not meant to be a sole alternative to other sensors as combination with lidar, radar or other data types (which was beyond the research scope) will probably enhance such multivariate forest structure modelling.

Radiative-transfer modelling has been used to simulate the reflectance and transmittance at various scales within the canopy (leaves, branches, crowns) in order to model LAI and other stand characteristics using image spectral information (e.g. Gastellu-Etchegorry et al., 1996; Leblanc and Chen, 1998; Peddle et al., 2004). This type of modelling is mostly limited to reflectance information. Perhaps more commonly used, empirical studies have been carried out in order to develop models that explain and predict structural attributes using spectral as well as spatial information extracted from the imagery.

Image texture, which represents spatial variation of image elements, has been used to provide increased explanation of forest structural attributes (e.g. Olthof and King, 1997; Wulder et al., 1998). For example, Wulder et al. (1998) incorporated first and second order (calculated from the grey level co-occurrence matrix (GLCM) (Haralick et al., 1973)) textural information into empirical models to predict LAI in the Fundy Model Forests in southeastern New Brunswick, Canada. This textural information was extracted 
from $1 \mathrm{~m}$ resolution multispectral airborne imagery. Their findings showed that image textural information, used alongside NDVI, provided a significant increased explanation of LAI.

Additionally, spatial dependence measures such as semivariance (e.g. St. Onge and Cavayas, 1997; Lévesque and King, 1999; Treitz and Howarth, 2000; Johansen and Phinn, 2006; Johansen et al., 2007) have been commonly used as predictors of forest structural attributes. Lévesque and King (1999) explored the use of the range of the semivariogram, which can indicate the distance at which pixels are no longer spatially correlated (often related to the size or scale of dominant objects in the imagery (Curran, 1988)), as well as the sill, which can indicate the total structurally dependent variance in the data (Johansen et al., 2007). Using variables extracted from multispectral airborne imagery, they investigated relations with forest canopy closure, stem density, crown size, tree height, and a tree stress index, at an abandoned acid mine site near Timmins, Ontario, Canada. Their results showed the value of such measures at the canopy scale using $50 \mathrm{~cm}$ and $1 \mathrm{~m}$ resolution imagery with respect to discriminating structural damage variations across the forest, however their conclusions stressed the importance of using multiple image spatial measures when modelling forest structural variability.

Radiometric fractions at the pixel level (e.g. Pellikka et al., 2000; Seed and King, 2003) or at the sub-pixel level using spectral unmixing (e.g. Hall et al., 1995; Peddle et al., 1999; Lévesque and King, 2003; Pasher et al., 2007) have also been used for modelling forest structural attributes. For example, Pellikka et al. (2000) used areal percentages of three levels of shadow (i.e. deep, between crown, and medium 
transitional) extracted from $25 \mathrm{~cm}$ resolution multispectral airborne imagery. In modelling LAI and canopy closure, they found regression models that incorporated shadow fraction variables provided greater explanation than those that included only spectral and GLCM textural information. Lévesque and King (2003) used linear spectral unmixing to decompose the spectra of mixed pixels into sub-pixel proportions of sunlit crowns, shadows, and wood in order to improve empirical models predicting forest structural attributes. They found that sub-pixel fractions, as well as spatial measures of the fractions, provided improvement over models that relied strictly on image spectral information.

\subsubsection{Modelling and Mapping Dead Wood Using Remote Sensing}

As previously discussed, dead wood is potentially an excellent indicator of biodiversity levels within a forest. Snags and CWD can be inventoried through sampling using a variety of methods (e.g. van Wagner, 1964; Bate et al., 1999; Valentine et al., 2001; Stahl et al., 2002; Jordan et al., 2004; Kenning et al., 2005). The non-uniform distribution of these structures in the forest (Ducey et al., 2002; Kenning et al., 2005) can require complex and intensive sampling strategies to properly survey an entire forest. Field methods are limited to point, line, or plot-based sampling and, as a result do not provide spatially continuous information at scales of interest for management. Modern harvesting and forest management regulations often require a certain density or volume of dead wood to remain (following timber removal) in order to provide habitat and support ecosystem sustainability (e.g. Franklin et al., 1997; Holloway et al., 2007). Additionally, forest conservation in areas not managed for timber production is often 
concerned with identification of areas of high biodiversity potential. A continuous map showing the locations and spatial distribution of dead wood across a forest could support such management and conservation goals as well as be used for habitat modelling and mapping of individual species.

\subsubsection{Direct Detection and Mapping}

Remote sensing can potentially offer cost effective and rapid methods for inventorying dead wood across a forest. The direct detection and mapping of standing dead wood has shown potential in coniferous forests, as well as in deciduous forests specifically for the detection of dying oaks caused by sudden oak death.

Butler and Schlaepfer (2004) created snag distribution maps from 1:10 000 Colour Infrared (CIR) air photos for spruce dominated forests within the sub-alpine zone in Switzerland. While accuracy results were very high, the research was carried out in sparse forests with isolated snags, and more importantly, it relied on manual photo interpretation and digitizing of dead trees, which was very time consuming. Others have used more automated methods, including Haara and Nevalained (2002) who developed a method to segment individual trees in 1:5000 CIR air photos of spruce dominated forests in southern Finland. For three forest stands, the average detection accuracy was $67 \%$ for dead and dying trees (61 - $100 \%$ needle loss). Leckie et al. (2004) used $60 \mathrm{~cm}$ resolution airborne multispectral imagery to detect and assess trees suffering from laminated root rot in Douglas fir forests in coastal British Columbia, Canada. They were able to successfully detect snags with fully intact crowns as well as those with missing branches using classification and automated tree crown delineation methods (Gougeon 1995). 
Several researchers have had success in mapping dead trees caused by sudden oak death in Californian hardwood forests. Kelly et al. (2004) evaluated three classification methods (unsupervised, supervised, and hybrid) applied to $1 \mathrm{~m}$ airborne multispectral imagery and achieved a best average accuracy of $96.3 \%$ using the hybrid classifier. Guo et al. (2007) developed a hybrid classification method combining object-based and knowledge-based classifications, which first segmented $1 \mathrm{~m}$ multispectral imagery into homogeneous objects and then used a set of rules to decide if each object was a dead crown (e.g. maximum size associated with known maximum crown size, compact shape, and adjacency to vegetation). Their results showed an accuracy of $96.1 \%$, compared to supervised classification with an accuracy of $86.8 \%$. Finally, Meentemeyer et al. (2008) were able to detect dead oak trees in $76 \%$ of sample field plots in the Big Sur ecoregion using an object-based classification and airborne colour $33 \mathrm{~cm}$ imagery.

\subsubsection{Indirect Modelling of Dead Wood}

While woody debris for the most part would not be directly visible by airborne or satellite sensors, indirect modelling of CWD has been conducted using image variables related to different aspects of forest structure that are manifested as image brightness variations. This is possible as a result of relationships that exist between dead wood and the surrounding forest structure, in particular the association of dead and dying trees with canopy gaps (Bursing, 2005; Kneeshaw and Prevost, 2007) or the presence of sparse tree crowns, which are visible in high resolution imagery.

In research carried out in Gatineau Park, Québec, Canada (the same forest used for the research reported in this thesis) following a major ice storm in 1998, King et al. 
(2005) developed a model indirectly predicting the number of downed branches in fifteen field plots using spectral and spatial variables extracted from $25 \mathrm{~cm}$ resolution colour infrared (CIR) photography (Adjusted $\mathrm{R}^{2}=0.71, \mathrm{p}<0.001$ ), standard error $=28.4 \%$ of observed mean ( 29.5 branches) in $20 \mathrm{~m} \times 20 \mathrm{~m}$ plots). In a mixed boreal forest in Northern Ontario, Canada, Olthof and King (2000) developed a forest structure index (FSI) using canonical correlation analysis of linear relations between multiple image parameters and field-measured forest structural parameters. The index, which incorporated spectral, textural, and radiometric image fraction information extracted from $50 \mathrm{~cm}$ multispectral imagery, was used to explain the variance in a set of field variables that included measurements of fallen dead wood. Pesonen et al. (2008) used an airborne laser scanner (ALS) to model woody debris volume in thirty-three field plots in Finland (Adjusted $\mathrm{R}^{2}=0.61, \mathrm{p}<0.001$ ) based on two predictor variables representing the intensity of the last laser pulse and height variation extracted from the first pulse (i.e. top of canopy). While the model showed a strong $\mathrm{R}^{2}$, it had an RMSE of $51.6 \%$ (14.09 $\left.\mathrm{m}^{3} / \mathrm{ha}\right)$.

Similarly, little research exists using remote sensing to indirectly model snags in the forest. Standing dead wood was included as a structural variable in the forest structure index research of Olthof and King (2000) as described above. Bater et al. (2007) used a set of lidar variables in order to predict the percentage of dead trees within field plots. In order to overcome skewed distributions, caused by the plots containing mostly live trees, they fit lognormal probability density functions to the frequency distributions of all the trees in the plots (including living and dead trees in nine decay classes), and found the mean of the resultant distribution to be highly correlated with the percentage of dead trees 
$(\mathrm{r}=0.88, \mathrm{p}<0.001)$. Their results showed good predictive capability of this parameter using lidar variables, with $\mathrm{R}^{2}$ ranging from 0.42 to 0.75 . Pesonen et al. (2008) also modelled snag volume (adjusted $\mathrm{R}^{2}=0.48 ; \mathrm{RMSE}=78.8 \%\left(14.74 \mathrm{~m}^{3} / \mathrm{ha}\right)$ ) using the same ALS data mentioned above, leading to the conclusion that air photos were probably more suitable than laser scanning for indirect detection of standing dead trees.

\subsubsection{Structural Complexity Modelling Using Remote Sensing}

While remote sensing has been widely, and quite successfully, used for modelling and mapping individual structural attributes, including dead wood, the literature includes limited examples of the use of remote sensing for modelling the multivariate aspects of forest structural complexity. Coops and Catling (1997a, 1997b) presented perhaps the first research that used remote sensing to model and map forest structural complexity. Using four band visual / NIR digital airborne videography data with a spatial resolution of $2 \mathrm{~m}$, they were able to model eucalyptus forest habitat complexity, which was measured in the field at study sites using Newsome and Catling's (1979) scoring system. A measure of local NIR band variance regressed against the field complexity scores resulted in an $R^{2}$ of 0.75 . Using the same dataset, this methodology was carried further in Coops et al. (1998) which incorporated automatic tree delineation to provide spectral and spatial statistics for individual tree crowns and crown clusters. Analysis of correlations of tree crown image statistics averaged across ten validation plots with the field-based complexity scores showed that the within-crown NIR variance was highly correlated $(r=$ $0.86, \mathrm{p}<0.001$ ) with the total habitat score, suggesting that as the structure of the forest became more complex the mean crown spectral variation increased significantly. 
Similarly, they found a strong negative correlation $(\mathrm{r}=-0.81, \mathrm{p}<0.001)$ with the average within-crown red reflectance, which they state was expected because in their environment the more complex the habitat, the more photosynthetically active the site and therefore the greater the absorption of the red wavelengths.

The complexity scoring system that they used included percent canopy cover, where increasing canopy cover related to increasing photosynthetic activity and was assumed to be linearly related with complexity. It is worth mentioning at this point that this may not hold true in all forest types. In other environments and depending on spatial scales used, complexity and species richness, have been shown to follow parabolic relations with productivity, with highest complexity and highest richness found at moderate productivity levels (e.g. Grime, 1979; Oindo and Skidmore, 2002; Schuster and Diekmann, 2005).

Building on earlier work by Jakubauskas (1996), Olthof and King (2000) used Canonical Correlation Analysis (CCA) in order to investigate multivariate relations between soil characteristics and forest structural measurements on the ground, as well as relations between structure and multispectral image variables in a mixed boreal forest near a large abandoned mine tailings deposit in Northern Ontario, Canada. Their analysis developed a health index for monitoring structural changes in the forest, and included spectral information from within-shadows along with textural information extracted from the imagery across their study plots. Cosmopoulos and King (2004) developed these methods further, also looking at temporal changes at the site using the structural index. They specifically examined linear relations between multiple forest structural variables 
and image variables using multispectral imagery with a spatial resolution of $50 \mathrm{~cm}$ and incorporated a variety of spectral, textural, and radiometric fraction image information into a CCA. An initial Principal Components Analysis (PCA) was used to reduce their original nine structural variables down to three components, representing $81.3 \%$ of the original variance. A PCA of the image variables resulted in four components representing $80.7 \%$ of the original variance. Their CCA resulted in a strongest correlation of 0.73 between the two sets of variables and their final regression model, which used the original image variables as predictors of the FSI, had an $\mathrm{R}^{2}$ of 0.61 . While their research produced a model linking FSI to the image variables, they concluded that the method could be improved by separate measurement of ground, intermediate, and overstorey structural information.

Looking at a range of regenerating conifer stands in Alberta, Canada, Wunderle et al. (2007) used PCA to create a single structural complexity index, representing the variation of structural attributes measured in forest plots ( $79 \%$ of the total variance). They regressed the index against a variety of spectral and spatial image variables extracted from pan-sharpened SPOT $(2.5 \mathrm{~m}$ panchromatic resolution and $10 \mathrm{~m}$ multispectral resolution) imagery and presented a three-variable regression model (Adjusted $\mathrm{R}^{2}=0.74, \mathrm{p}<0.001$ ) that, once applied spatially, was validated using a withhold-one jack-knife approach. This approach showed that the model generally overestimated the actual index value, a result that was attributed to the fact that stands of less than five years of age (i.e. recently cut) had several parameters equal to zero (e.g. crown closure, stem density, average dbh). 
Sampson et al. (2001) presented perhaps the first known research that incorporated a Redundancy Analysis (RDA), which is similar to a combined PCA and CCA analysis (to be discussed in detail in Section 4.3.4) in order to investigate relations between forest structural variables and image variables. Their research investigated the potential for differentiating between three silviculture practices in a hardwood forest north of Sault Ste. Marie, Ontario, Canada. They used a variety of spectral indices derived from CASI (Compact Airborne Spectrographic Imager) imagery that were related to forest structure and function (i.e. potentially related to pigment concentration and chlorophyll fluorescence). As a final analysis method, the authors ran an RDA between the set of field variables (including canopy openness, crown area, and basal area of overstorey and understorey components) and image variables. In doing so, they were able to create ordination diagrams to help explore the relation between field and image variables. Their results showed significant, but admittedly weak, relations, which were held together by the inclusion of the clearcut plots in the analysis, resulting in the RDA being completely dependent on the canopy openness variable for most of the explanation of variance in the field data.

de la Cueva (2008) presented further relevant research, using RDA to evaluate the relationships between structural variables (canopy closure, vegetation cover, basal area, stem density, and stand height) and fourteen image variables consisting of spectral indices derived from Landsat 7 ETM+ imagery, along with topographic variables. Unlike the previously discussed research of Sampson et al. (2001), this analysis focused solely on the use of RDA and presented extensive results of the analysis. This research was carried out in order to differentiate between three different types of vegetation in forests 
in western Spain, which were dominated by conifers, evergreen sclerophyll, and deciduous trees. RDA was carried out separately for each type of vegetation. The results reported the best five explanatory image variables for each RDA model using a forward selection method. They showed a maximum explanation of the field variance of $24 \%$ (ranging from 6 to $24 \%$ ) using a combination of the first and second RDA axes, which was lower than the author had expected.

\subsection{Justification for This Research}

Following an extensive review of the literature provided in the previous sections, it was clear that many possibilities exist in order to improve multivariate modelling of forest structural complexity and mapping forest dead wood distribution using remotelysensed imagery in a semi-automated manner.

It must be clarified that while this research was set within the context of habitat and biodiversity conservation, it did not focus on or involve the direct measurement, modelling, mapping, or validation of biodiversity itself. Rather, this research relied on prior theoretical and field-based research, as presented in the background section, which demonstrated the importance of structural complexity and keystone structures, such as CWD and snags, for the healthy functioning of forest ecosystems and specifically as potential indicators of diversity for many groups of species within the forest. This research in itself did not attempt to directly relate biodiversity levels in the forest to the measured structural attributes or the resultant mapped indicators. 
Early work by Coops and Catling (1997a, 1997b) and Coops et al. (1998) involved the use of a single structural complexity index derived through subjective scoring of five aspects of forest structure. Other work using various ordination techniques (i.e. PCA, CCA, RDA) avoided the need for subjective scoring (Jakubauskas, 1996; Olthof and King, 2000; Sampson et al., 2001; Cosmopoulos and King, 2004; Wunderle et al., 2007; de la Cueva, 2008). This thesis builds on this existing work, presenting new methods of modelling structural complexity using the RDA approach. The inclusion of image spectral and spatial information extracted from within tree crowns and shadows, as opposed to simply relying on plot-based averaged information, along with the use of object-based information representing canopy and shadows in the forest, represents a comprehensive set of predictor variables that has yet to be fully explored for modelling structural complexity. This set of variables was felt to represent various aspects of forest structure, as manifested in the imagery, and using them simultaneously provided an excellent opportunity to examine what types / sources of image information could best be used to explain field-based structural complexity. The selection of predictor variables was done based partially on issues raised in the literature, specifically the need for high resolution imagery that permits the use of necessary detailed spatial information (e.g. de la Cueva, 2008), as well as the use of object-based information (e.g. Sampson et al., 2001).

With respect to the direct use of RDA, in the past it has only been used as an exploratory, or descriptive, technique. The methods presented in this thesis demonstrate the full potential of RDA by using it as a direct means for deriving an empirical model that can be applied spatially in order to map predicted structural complexity. As well, 
methods presented include important intensive validation of both the modelling and mapping phases of the research.

With respect to indirect modelling and mapping of downed dead wood, the literature was found to be lacking (King et al., 2005; Pesonen et al., 2008) suggesting that further exploration into the possibility of using high resolution multispectral imagery for indirectly modelling and mapping forest dead wood was necessary. The inclusion of a wide variety of image variables was thought to provide potential improvement on the results of previous research by representing different aspects of the forest structure related to the presence of downed dead wood, which had yet to be investigated.

Similarly, existing research investigating the direct detection and mapping of snags was limited, and all existing work in hardwood forests was focused exclusively on mapping sudden oak death in Californian forests (Kelly et al., 2004; Guo et al., 2007; Meentemeyer et al., 2008). As a result, part of this research investigated the potential for developing semi-automated methods of detecting and mapping dead wood, making full use of very high resolution imagery to map fully intact dying crowns as well as partially intact crowns and individual dead branches in the canopy. 


\subsection{METHODS}

This research investigated the development of two related structurally-based indicators of biodiversity that could be derived and mapped from high resolution airborne imagery: (1) forest structural complexity, and (2) the spatial distribution of dead wood across the forest. The study area and the airborne imagery that was collected and processed were used for both indicators so they are only described once. Any image processing and variable extraction carried out for only one of the two indicators has been included in the thesis sections for that indicator where appropriate.

\subsection{Study Area}

This research was carried out in Gatineau Park, which extends northwest across the Ottawa River from the city of Ottawa. The park is located in the foothills of the Laurentian Mountains, and the underlying geology of the Park consists mostly of the hard igneous Precambrian rocks that form the Canadian Shield (NCC, 2005; Red Path Museum, 2006). The southern section of the Park lies on more recent sedimentary deposits of the Ottawa limestone formation (Lopoukhine, 1974). The St. Lawrence Lowlands lie in the shadows of the Gatineau Hills, with the clearest division seen by the $300 \mathrm{~m}$ cliffs of the Eardley Escarpment, which marks the southeastern boundary of the Canadian Shield. The Park is best described as being rolling hills that are a result of glacier activity.

Currently, more than $80 \%$ of the Park's approximately 36000 hectares are forested, with the overstorey in the southern portion of the Park dominated by sugar 
maple (Acer saccharum Marsh.), with small patches dominated by American beech (Fagus grandifolia Ehrh.), trembling aspen (Populus tremuloides Michx.), and red oak (Quercus rubra L.). Small numbers of red maple (Acer rubrum L.), American basswood (Tilia americana L.), ironwood (Ostrya virginiana (Mill.) K. Koch), white ash (Fraxinus americana L.), black ash (Fraxinus nigra Marsh.), white birch (Betula papyrifera Marsh.), and black cherry (Prunus serotina Ehrh.) are also present. This composition is partially a result of the historical logging of white pine (Pinus strobus) that occurred in the area during the late nineteenth and early twentieth centuries, which allowed the secondary growth hardwood forest to take over (Lopoukhine, 1974; King et al., 2005; NCC, 2005).

This forest was selected following successful ice storm damage modelling and mapping in the Park using remote sensing (King et al., 2005). A small area of the forest was selected for this study that stretched approximately $4.5 \mathrm{~km}$ from just south of Champlain Lookout northwards to Meech Lake. This area was aligned with one of the sampling transects from previous studies (King et al., 2005; Pellikka et al., 2000). It was approximately $400 \mathrm{~m}$ wide ( $180 \mathrm{ha}$ ) (Figure 1$)$, with the elongated study area setup to minimize the number of flight-lines needed for image acquisition. This was critical as a result of the small view angle used.

The study area contained a range of elevations, slopes, and aspects, and it had experienced patchy damage from the 1998 ice storm. Both topography and ice storm damage were expected to contribute to forest structural variation. 


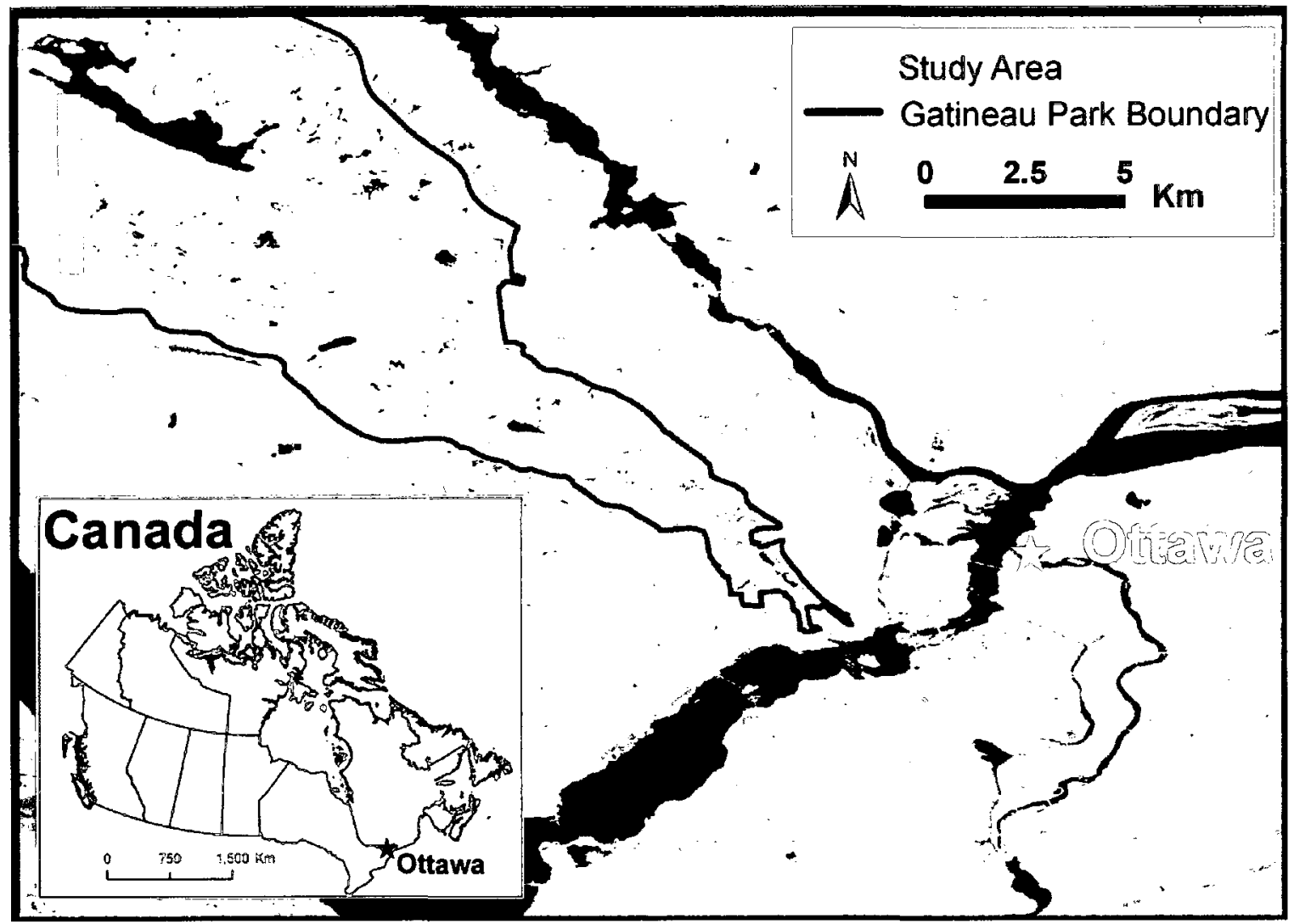

Figure 1. Location of study area within the southern section of Gatineau Park near Ottawa, Canada. Shown with 2005 SPOT satellite image in the background. (Courtesy P. Pellikka, University of Helsinki and ESA).

\subsection{Airborne Imagery Collection and Processing}

On August $21^{\text {st }} 2007$, three-band (green $500-600 \mathrm{~nm}$, red $600-700 \mathrm{~nm}$, near infrared $750-850 \mathrm{~nm}$ ) imagery was acquired over the study area using a DuncanTech MS4100 camera with a Nikon $24 \mathrm{~mm}$ lens mounted to acquire vertical images using a modified door attached to a Cessna-172 aircraft (Figure 2). The flight altitude was $650 \mathrm{~m}$ in order to achieve $20 \mathrm{~cm}$ nominal spatial resolution, which was selected based on previous forest structure research in the park as well as the desire to detect individual forest objects, including dead branches. Imagery was acquired flying from South to North 
along two flightlines (Figure 3), resulting in eighty-eight images with $\sim 30 \%$ forward and side overlap covering the study area.

Each image was cropped to 1080 pixels x 1080 pixels $(\sim 216 \mathrm{~m}$ x $216 \mathrm{~m}$ ground coverage), representing a maximum view angle of $9.5^{\circ}$ in all directions. This angle was selected based on the research of Seed and King (1997), who found significant negative effects of view angles beyond $10^{\circ}$ on forest structure modelling. The imagery was acquired between 1:37-1:48 pm, just after solar noon, with solar zenith and azimuth angles of $33.7^{\circ}$ and $190.2^{\circ}$, respectively. The sensor system allowed image histograms to be viewed in-flight so exposure times were manually set for each band over adjacent forested areas before acquiring the data. This was done in order to maximize the dynamic range for forests while avoiding saturation in the imagery. The sky was completely cloudfree during image acquisition. 


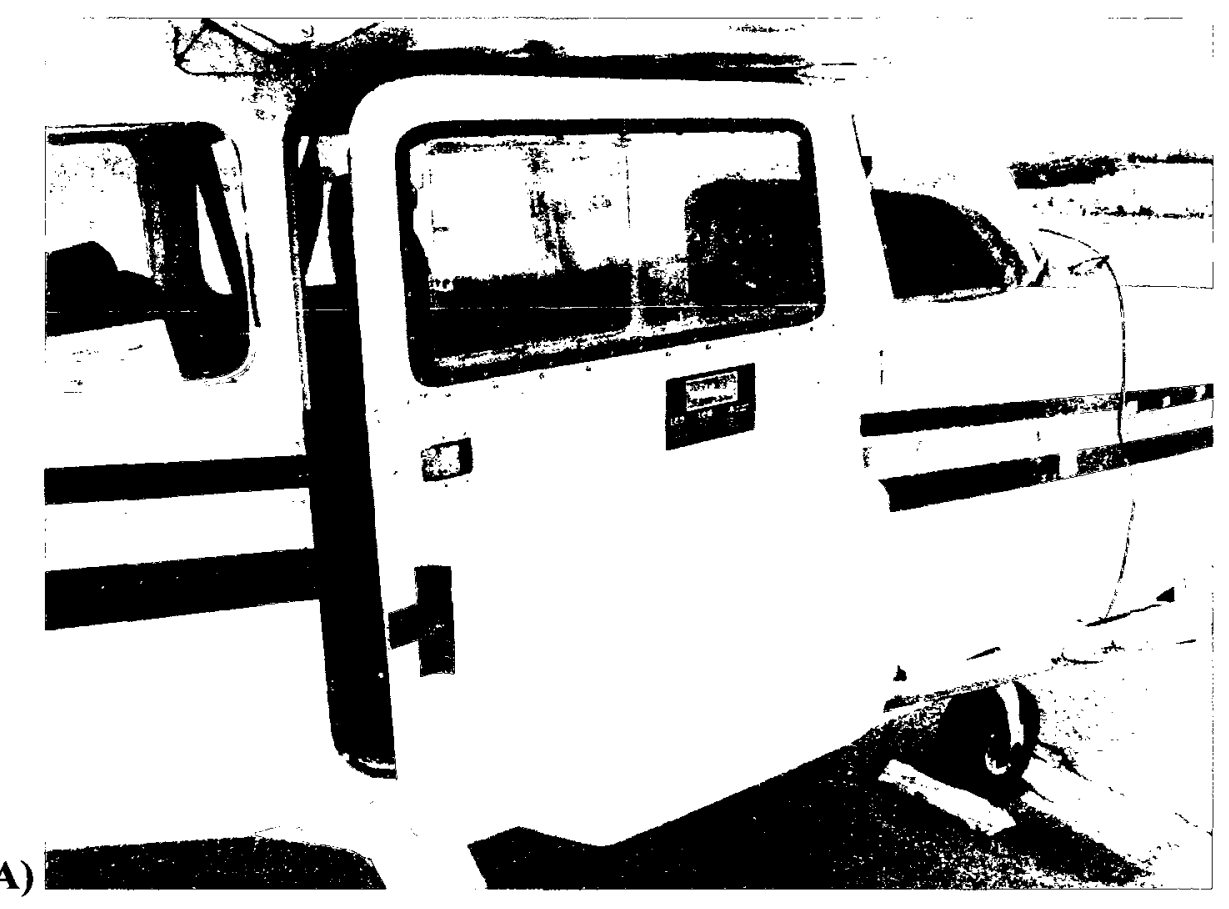

(A)

(B)

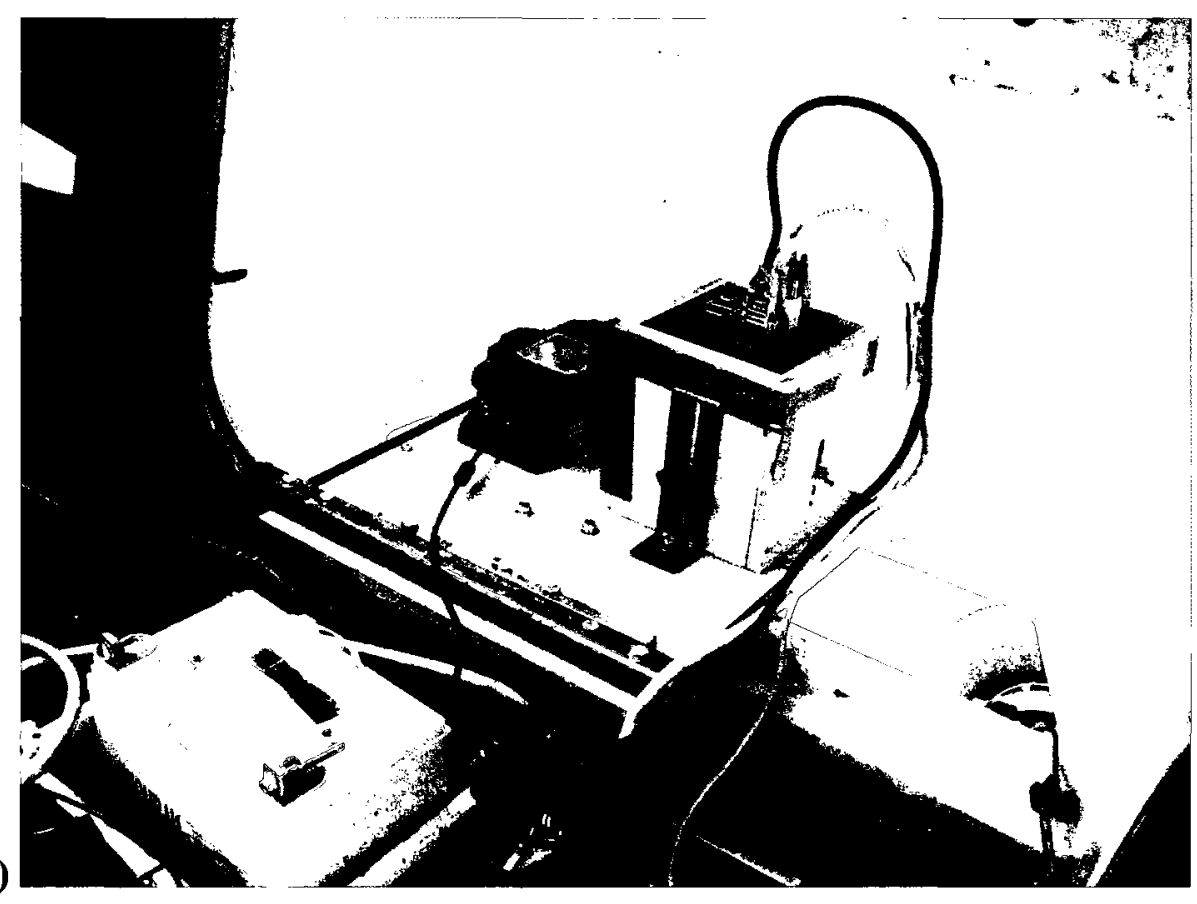

Figure 2. Modified door on Cessna-172 to allow vertical view angle (A) and digital camera setup within the pod of the door (B). Note that a second RGB digital camera is shown mounted in the image, which was being tested at the time. 


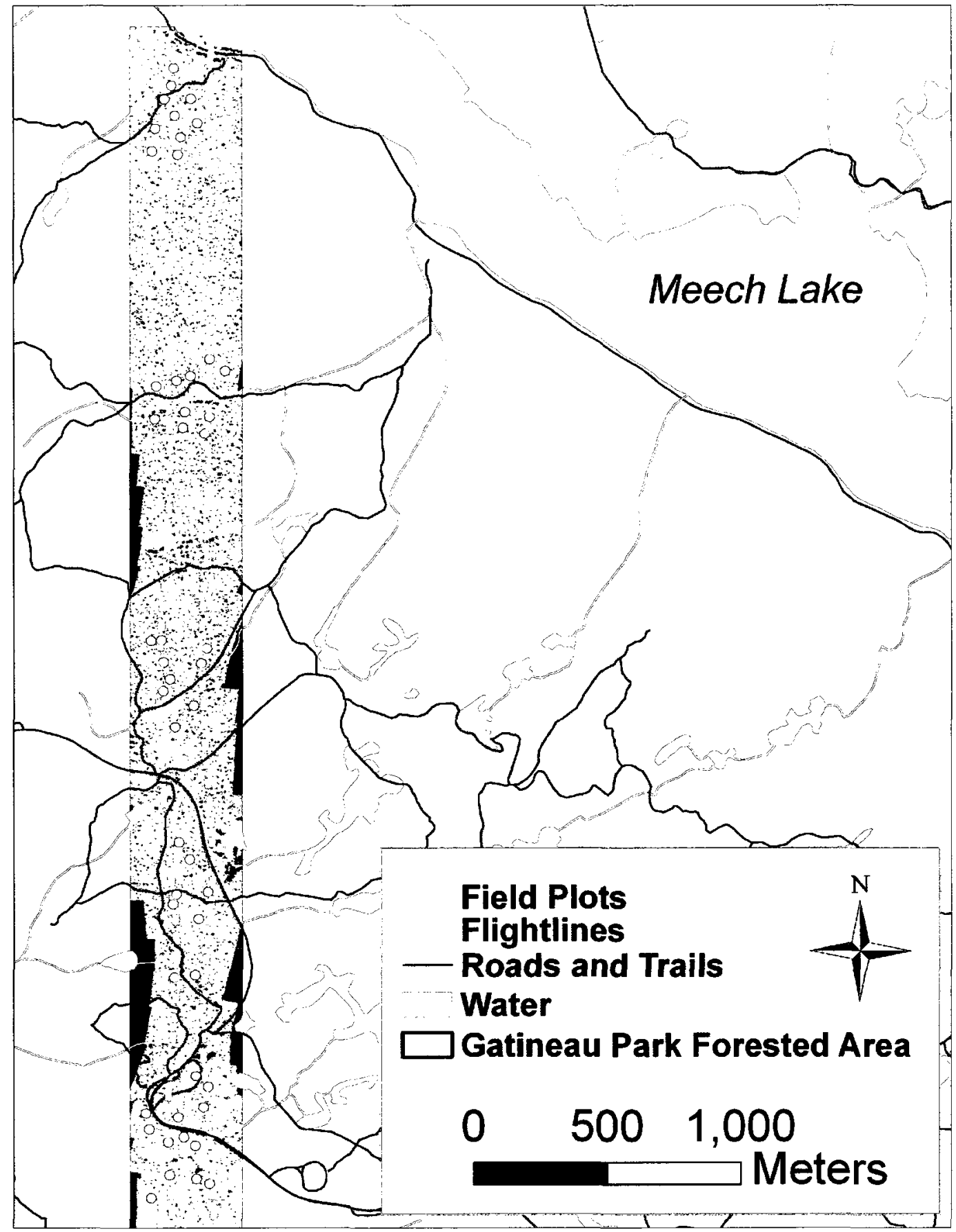

Figure 3. The final multispectral image mosaic shown within Gatineau Park covering the study area along with the two flight-lines used to acquire the imagery. The fifty field plot locations are also shown. 
Typically, airborne imagery has brightness variations caused by sun-sensorsurface geometry (bi-directional reflectance variation (BRV) and topographic variations) as well as lens effects (light fall-off / optical vignetting) and in order to better represent the actual surface reflectance these variations must be corrected. BRV effects were reduced by limiting the view angle (i.e. $9.5^{\circ}$ ) Additionally, the spatially non-uniform camera / lens response was reduced through lens calibrations (Geospatial Systems Inc., 2007). Despite these adjustments, some brightness variations were still noticeable in the imagery. In all images, discovered through visual assessment as well as extracting profiles across the images at various places, brightness was found to increase from the bottom to the top of the imagery (in the direction of flight and plane of the sun). For example, from a few sample images acquired over flat terrain, the average brightness (in digital numbers, DN) extracted from tree crowns at the top of an image was found to be $\bar{x}$ NIR $=174, \bar{x}_{R}=38$, and $\bar{x}_{G}=163 \mathrm{DN}$, while crown pixels extracted at the bottom of the same images in the same forest had average brightness values of 164,28 , and $160 \mathrm{DN}$, respectively. Additionally, topographic brightness variations were also seen specifically between north and south facing slopes, which were the dominant aspects in this area. Sampled crowns extracted from opposite sides of a valley showed that the south facing slope had brightness averages of 185,29 , and $147 \mathrm{DN}$ for the NIR, R, and G bands, respectively, while the opposite north facing slope was darker with averages of 166,23 , $125 \mathrm{DN}$ for the same bands. In order to compare different areas of the imagery to each other and to develop models of structural complexity from image brightness and spatial information, these residual brightness variations needed to be removed. 
A variety of methods have been widely used to correct for BRV in imagery such as empirical methods (Pickup et al., 1995; Pellikka, 1998), theoretical BRDF modelling methods ( $\mathrm{Li}$ and Strahler, 1992; Omari et al., 2008) and topographic variations such as the cosine correction, c-correction, Minnaert correction, and statistical-empirical corrections (e.g., Teillet et al., 1982; Meyer et al., 1993; Soenen et al., 2005). However, in tests using 2005 imagery collected with the same camera over the same forest of this research, Bemrose (2006) found that many of these methods overcorrected the imagery, causing flare-ups towards the edges. His research showed that an empirical net brightness correction can be more effective in reducing overall brightness variations across an image scene of uniform land cover. A net image correction can also be applied in one step, eliminating the need for separate BRV, sensor / optical, and topographic correction algorithms. For example, Richter (2005) presented a correction method that adjusted pixel brightness by comparing columns of pixels across the image to pixels in the nadir region (used by Bemrose (2006)). Additionally, Cosmopoulos and King (2004) implemented a column-based polynomial fit inversion method. The net correction methods implemented by Bemrose (2006) and Cosmopoulos and King (2004), as well as the c-correction method (Teillet et al., 1982) were tested for use in this research, however, the results were not satisfactory and while they removed brightness variations from some areas of the images, they were inconsistent and overcorrected many areas. Instead a simple net correction was developed and implemented as described below (included in Appendix 1).

Non-forest was masked out of each image, and the general brightness trend across each image (for each spectral band independently) was calculated by passing a large 
window average filter ( $99 \times 99$ pixels) across each image. This image was normalized from 0 to 1 and inverted to create a correction template. Each image was corrected by multiplying it by its corresponding template to create images with uniform brightness across the field of view (Figure 4). The images were then histogram matched to a set of master images located over flat terrain using an algorithm based on Nielsen (2004), which matched the cumulative histogram of an image to that of the master images. This was done in order to slightly adjust the overall brightness such that it would match that of the master images' (e.g. Cowan and Cooper, 2008).

As a final check of the quality of the brightness correction and mosaic procedure, crowns and shadows were examined at different locations across the mosaic. First, crown and shadow pixels were extracted at nadir and off nadir, and second, pixels were extracted on flat areas at nadir as well as on south facing and north facing slopes, again at nadir. For all three spectral bands, results of this analysis showed that there were no significant differences $(\mathrm{p}<0.001)$ between $\mathrm{DN}$ values taken at different places across the images for either crown or shadow pixels. As well, while the correction procedure left the images looking spectrally "flat", this was simply a result of the view scale (e.g. Figure 4). However, when zoomed in to a scale where individual trees are visible, it was evident that the within and between crown variance remains and was not removed by any correction processing (Figure 5).

Following this correction procedure, the eighty-eight images were mosaiced together (positional RMSE $<5$ pixels $(\sim 1 \mathrm{~m})$ ). In the overlap region between each image pair, the image data representing radiance backscatter were retained in the mosaic. This 
approach followed that of Seed and King (2003), who presented better modelling results for LAI using backscatter portions over forward scattering portions of each image.

The mosaic was manually georeferenced to two $2004,25 \mathrm{~cm}$ leaf-off orthophotos using a thin plate spline model, which fit to the input ground control points exactly. An RMSE of $2.56 \mathrm{~m}(\mathrm{X})$ and $2.29 \mathrm{~m}(\mathrm{Y})$ was calculated using a set of independently collected test points to assess the fit between the mosaic and the 2004 orthophotos. The georeferencing error, combined with that of the mosaic procedure $(\sim 1 \mathrm{~m}$ error) and the GPS positioning error of field plot locations $(\sim 1 \mathrm{~m})$, resulted in a combined RMSE of $\sim$ $3 \mathrm{~m}$ in both the $\mathrm{X}$ and $\mathrm{Y}$ directions, or approximately $15 \%$ of the plot size. This error was considered to be small enough to not affect extraction of image information from the plots.

The image processing effectively corrected brightness variations (final mosaic shown in Figure 3 and Figure 6), however, it was not meant to model variations in structural complexity due to topography. Instead, topographic data were used directly for this purpose as described in Section 4.3.3.4. 


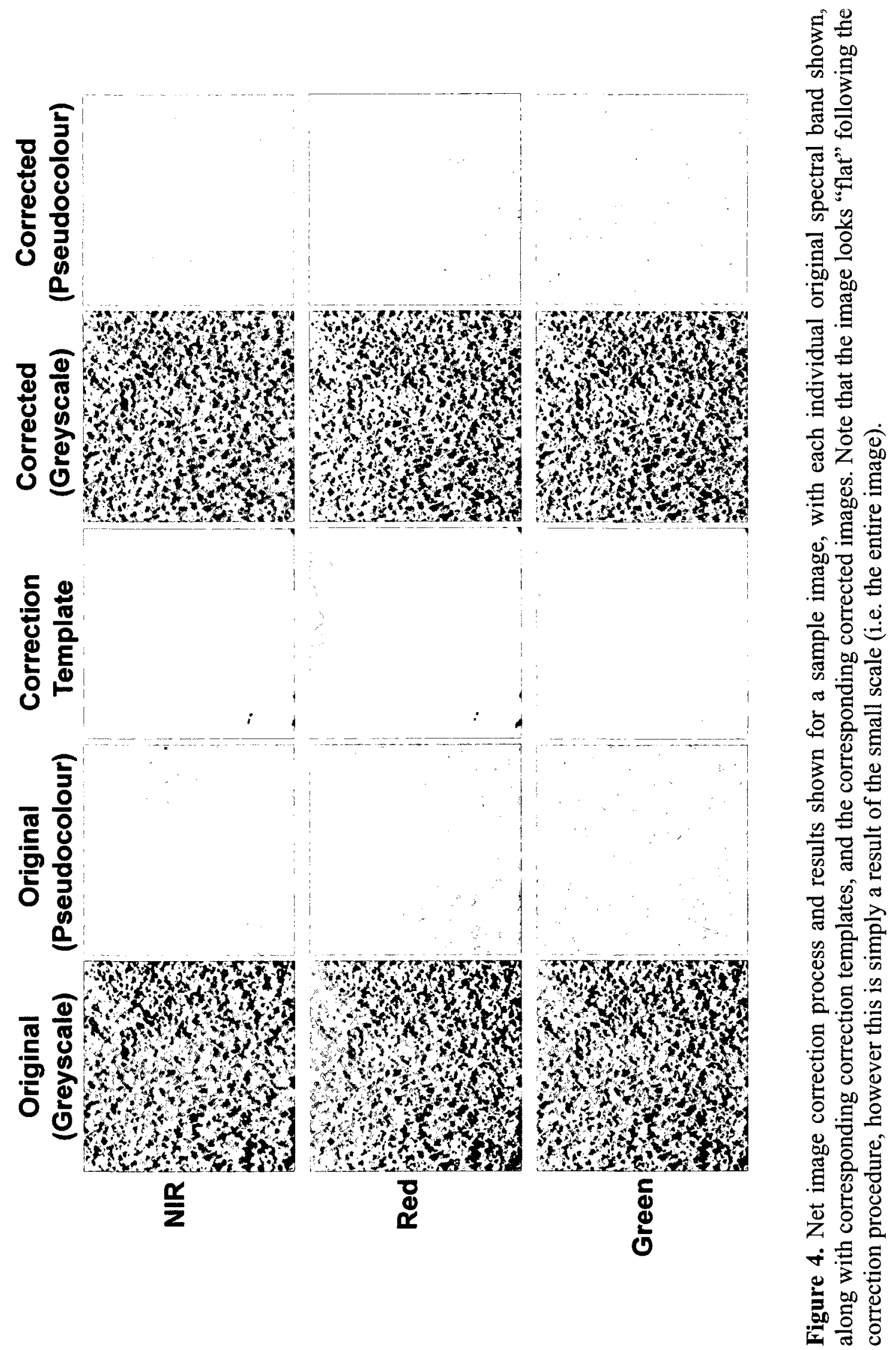




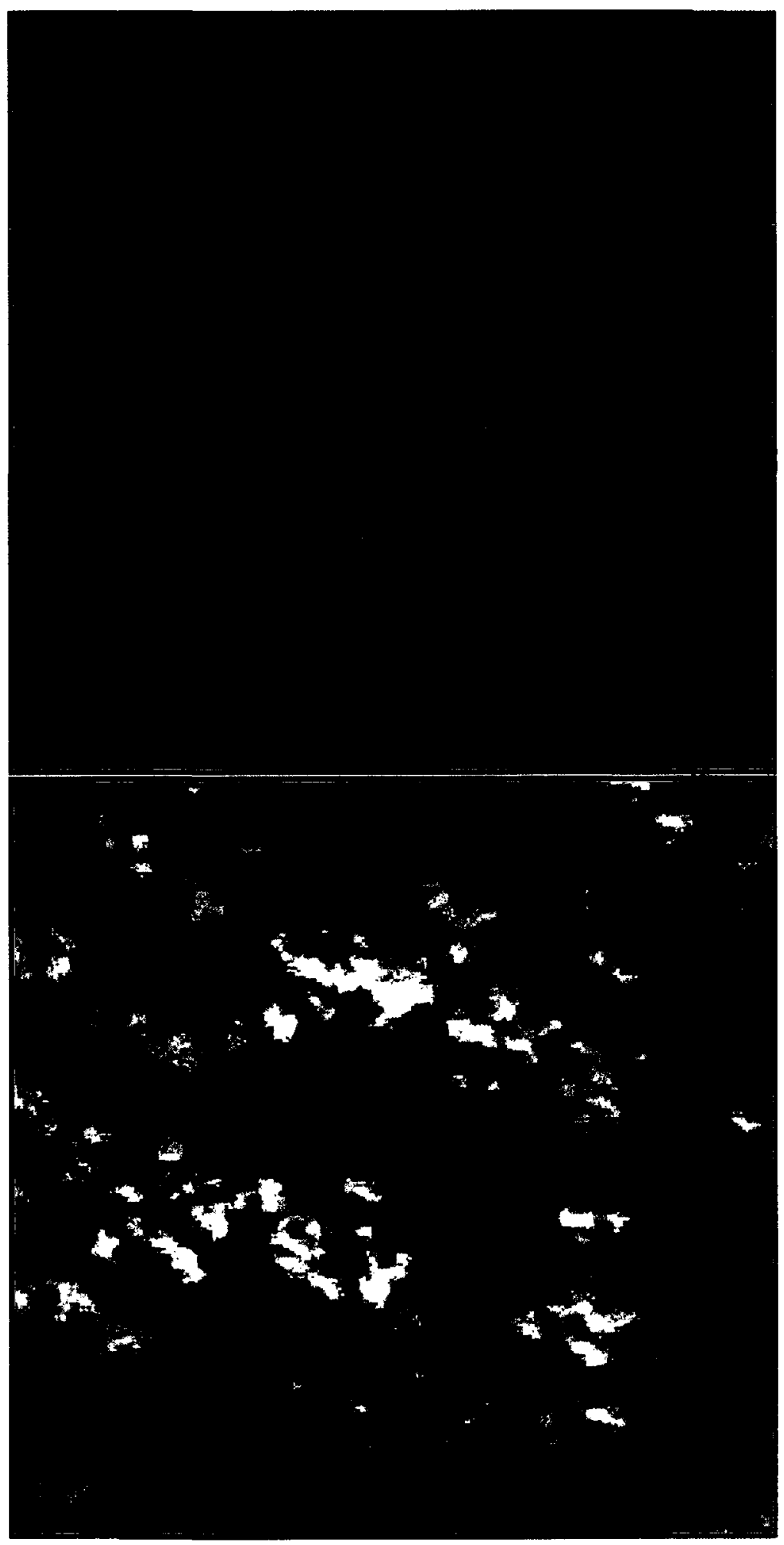

Figure 5. A zoomed in $50 \mathrm{~m} \times 50 \mathrm{~m}$ example of the corrected imagery showing the within and between crown variance, which was still present in the imagery following brightness correction processing. CIR and NIR (greyscale) images are shown. 


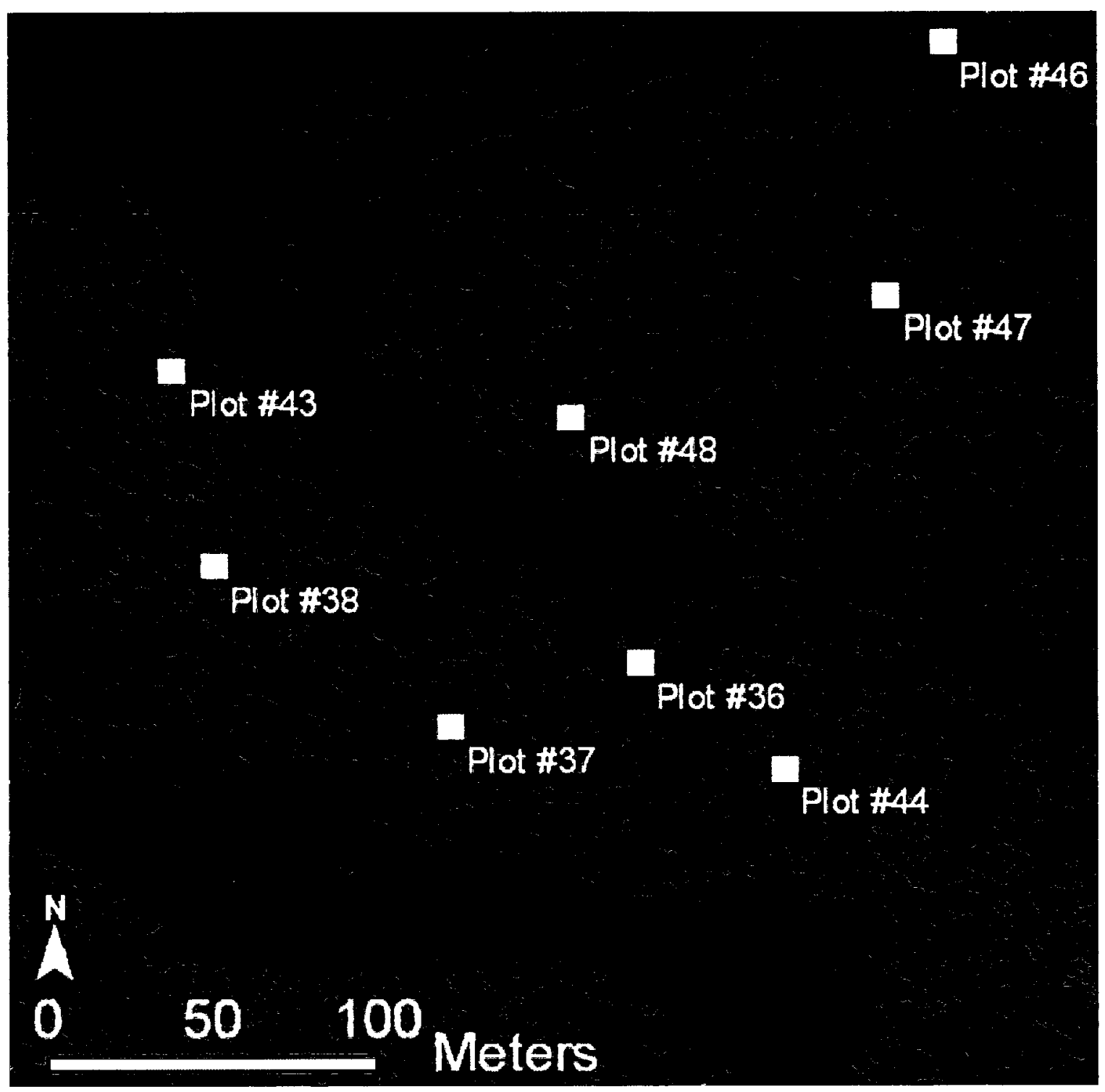

Figure 6. A subset of the multispectral image mosaic, spanning across the seams of multiple images. Locations of some of the 50 field plots used for the development of a structural complexity index are shown within this area. 


\subsection{Development of an Image-based Structural Complexity Index}

\subsubsection{Field Plots}

In the summer of 2007 , once all the trees had fully leafed out, locations for fifty field plots were selected across the study area. Since structural complexity was thought of as a continuous gradient, plots were not only selected to represent the extreme conditions (high and low complexity), but rather to represent the full range of structural and topographic conditions found within the forest. The overstorey tree size and position, canopy closure, understorey and ground vegetation, as well as the amount of standing and fallen dead wood were initially qualitatively assessed before a plot was established. The majority of the plots were selected to have structural complexity in the middle portion of the range observed in the field. The remaining plots split between areas with visually higher and lower structural complexity levels, which were known to be less frequently occurring and much smaller in size compared with medium complexity areas. As a result of the size differential between general complexity levels, it was felt that a random sampling approach for plot establishment would not have worked and instead purposeful sampling was used, which ensured that the extreme conditions were represented. Having this full range of conditions was obviously considered important for producing explanatory models that were representative of the conditions found in the study area.

At each site a $20 \mathrm{~m} \times 20 \mathrm{~m}$ field plot was established and all measurements were taken, including acquiring GPS positions for each plot in order to locate them within the airborne imagery. Plot sizes were chosen for continuity with previous forest structure 
research in the park (King et al., 2005) and based on research on optimal plot extent for sample-based spatial image analysis in this forest (Butson and King, 2006) as well as analysis of the spatial variance of ground measurements in the forest that showed the semivariance range of tree height, $\mathrm{dbh}$, and crown size to be less than $20 \mathrm{~m}$ (Butson and King, 1999). The plot size was also expected to be much larger than combined GPS and image registration positional errors.

The coordinates of the four corners and centre of the plots were collected using a Trimble GeoXT real-time differential (WAAS) GPS with approximately $1 \mathrm{~m}$ horizontal accuracy following post-processing using the Gatineau SOPAC base station (CAGS) located within $10 \mathrm{~km}$ of the study area.

\subsubsection{Structural Attribute Measurements}

The following sections describe the field measurements taken in each plot, with each measurement selected based on previous structural complexity research as well as their expected manifestation in the imagery, either directly (e.g. crown size, canopy closure) or indirectly (e.g. large CWD not always visible in the imagery but often associated with overstorey gaps).

\subsubsection{Overstorey Tree Size and Distribution}

Overstorey trees, which were considered to be those with a diameter at breast height $(\mathrm{dbh})>10 \mathrm{~cm}(\mathrm{CFIC}, 2004)$ as measured at a height of $1.3 \mathrm{~m}$, were measured in terms of their presence, size, and horizontal distribution. These trees formed the upper canopy of the plots and were expected to be visible in the imagery and / or directly 
influence the light conditions on the ground. Each tree's dbh, height (using a clinometer), and crown diameter (estimated by averaging together the major and minor perpendicular axes on the ground directly beneath the crown edge) were measured. The basal area of each plot, which can be related to stand density, was calculated using the live trees' individual basal areas along with the area of the plot.

The average dbh, height, and crown diameter were calculated for each plot. More importantly, the standard deviation of dbh representing tree size variance was calculated. This measure is thought to be one of the most useful individual structural measures related to diversity of micro-habitats (Neuman and Starlinger, 2001; McElhinny et al., 2005). The variation in tree heights was calculated as an indicator of how much vertical stratification existed (Van den Meersschaut and Vandekerkhove, 1998; Zenner, 2000) in accordance with MacArthur and MacArthur (1961)'s original observations of the relation between bird diversity and vertical stratification. Additionally, the number of large trees in each plot, which is associated specifically to habitat availability, both while still living and eventually when potentially transformed to large snags (Spies and Franklin, 1991; Van den Meersschaut and Vandekerkhove, 1998; Vaillancourt et al., 2008), was calculated as the number of trees beyond the $90^{\text {th }}$ size percentile $(\mathrm{dbh}>35 \mathrm{~cm})$ of all trees measured in all plots.

The horizontal distribution of trees, calculated based on the inter-tree nearest neighbour distance can be used to describe the arrangement and distribution of overstorey trees (Pretzsch, 1997; Neumann and Starlinger, 2001). The average distance between trees was also calculated, having been shown to be a useful structural indicator (Spies and 
Franklin, 1991). Indices such as the Clark-Evans Index (Clark and Evans, 1954) or the Pielou Index of non-randomness (Pielou, 1959), as introduced in 3.1.2.3, were not used since indices are often difficult to interpret compared to more simple statistical measures calculated from the data's distribution.

\subsubsection{Canopy Openness and LAI}

Canopy openness and leaf area index (LAI) are considered common indirect measures of canopy structure (Chen and Cihlar, 1996; Frazer et al., 2000). They provide information related to productivity and light conditions in the forest, which impact the understorey and ground vegetation (Canham, 1988; Lhotka and Loewenstein, 2006), and can greatly affect stand regeneration, structure, and productivity (Englund et al., 2000). Digital hemispherical photographs, which have been widely used to derive LAI and openness (Leblanc et al., 2005), were taken in each plot using a Nikon Coolpix 8800 (8 megapixels) with an FC-E9 fisheye lens ( $180^{\circ}$ field of view) under diffuse sky conditions in order to minimize direct scattering of sunlight off leaves and trunks as well as avoid difficulties in defining vegetation edges in the photograph (Olthof et al., 2003). Previous tests in the forest showed that one photo at each plot centre would be sufficient for representing the general structure of the plot. Gap fraction calculated at a test plot centre as well as at locations halfway between the centre and the plot corners (a total of five photos) showed a standard deviation of less than $10 \%$, which was felt to be an insignificant variation given the application. Photos were taken with tripod heights of $1 \mathrm{~m}$ and $6 \mathrm{~m}$ in order to provide vertical structural information (Meir et al., 2000; Soudani et al., 2002). Photographs taken at a height of $6 \mathrm{~m}$ using an extendable pole that was raised 
to the desired height and followed by leveling of the camera (E. Seed and D. King, unpublished data).

Processing of the hemispherical photographs was carried out using DHP.exe (v. 4.5.2.) and Tracwin.exe (v. 4.1.1.) (Leblanc, 2006), which, following Leblanc et al. (2005), calculated LAI directly from hemispherical photographs $\left(55^{\circ}-60^{\circ}\right.$ zenith angle) using the CLX clumping index (Leblanc et al., 2005) to adjust for the non-random distribution of the vegetation structure. Canopy openness, or percent open sky, (Frazer et al., 2000) was calculated using an integration of the gap fraction across the zenith angles from $0^{\circ}-60^{\circ}$ (following Miller (1967) and S. Leblanc, pers. comm., 2008).

\subsubsection{Understorey and Ground Vegetation}

In temperate hardwood forests, unless a major gap was created that allowed penetration of direct sun irradiance, understorey and ground vegetation are generally not directly visible or measurable from airborne imagery. However, there is a direct connection between vegetation abundance and structure below the upper canopy with the overstorey structure, in terms of canopy gaps and light penetration (Canham, 1988; Olthof et al., 2003), and therefore understanding the vertical structure of the understorey can provide additional useful information contributing to the quantification of the overall complexity of the plots (Spies and Franklin, 1991; McElhinny et al., 2006). A lineintercept method was used, running along the diagonals of the field plots $(2 \mathrm{x} \sim 28 \mathrm{~m}$ long), with a survey of the vegetation height sampled every $50 \mathrm{~cm}$ along the lines providing 112 samples for each plot (Figure 7). Vegetation was broken down into five height classes: $<10 \mathrm{~cm}$ (herbs and low grasses); $11-50 \mathrm{~cm}$ (ferns, tall grasses); $51 \mathrm{~cm}-1$ 
$\mathrm{m}$ (seedlings); $1-2 \mathrm{~m}$ (shrubs); $>2 \mathrm{~m}$, but $\mathrm{dbh}<10 \mathrm{~cm}$ (understorey vegetation). This procedure adhered to methods outlined for the same forest in a study by Pisaric et al. (2009). The highest vegetation present at each sample point was recorded, providing an overall picture of vertical heterogeneity across the plots, as opposed to recording multiple heights at a single location. Percentage coverages for each height class were later calculated, representing the percentgage of the plot covered by ground / understorey vegetation of that height.

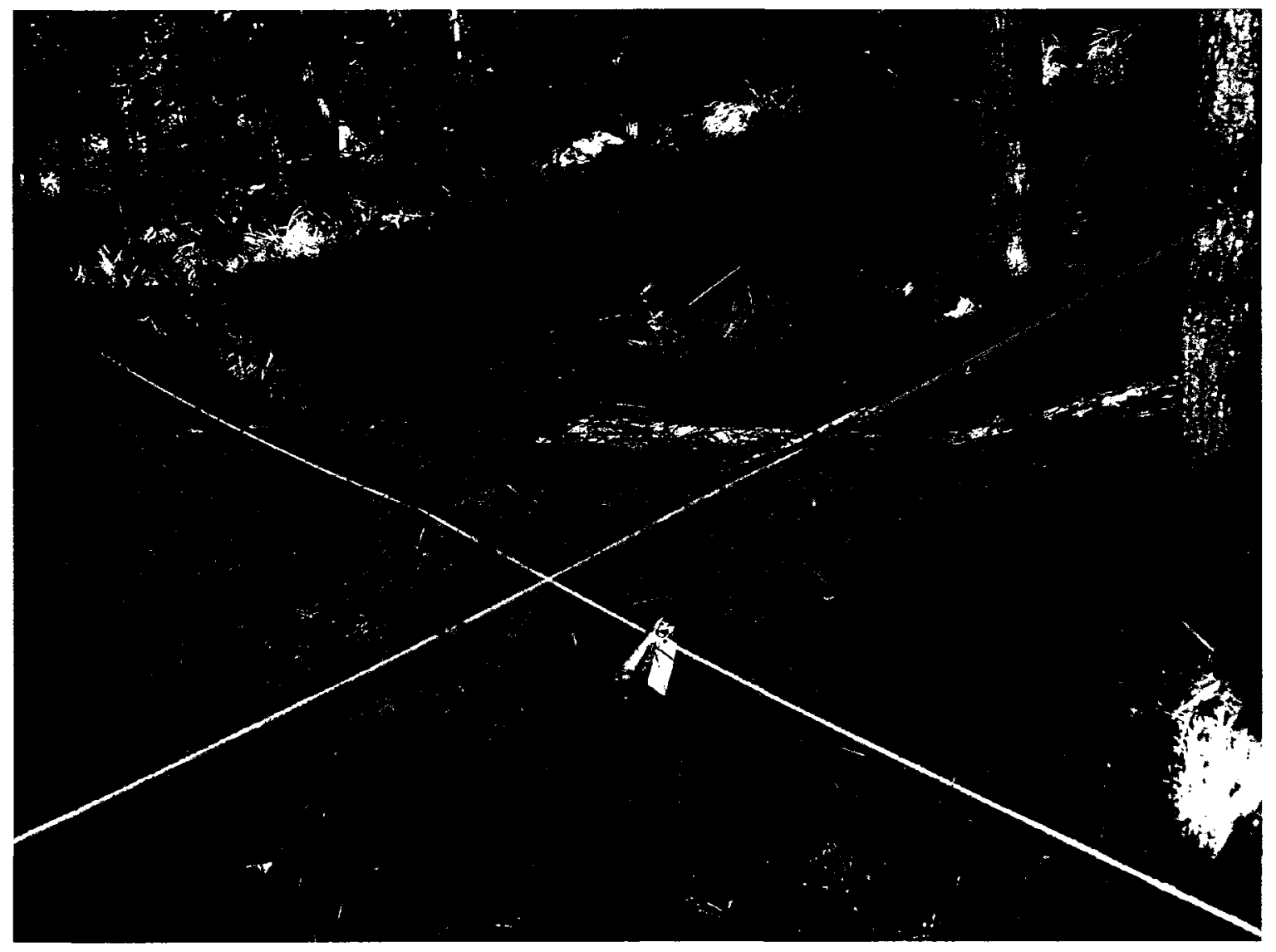

Figure 7. Example of field plot setup, with marker at the plot centre and ropes used to mark the diagonals. Understorey and ground vegetation were sampled along these ropes at every $50 \mathrm{~cm}$ (at each orange mark) and coarse woody debris was measured. This was done in order to estimate volume, wherever a piece was crossed by the rope. 


\subsubsection{Dead Wood}

Dead wood was measured in each plot in terms of snags (i.e. dead standing trees) and coarse woody debris (CWD), based on their potential to be used as bioindicators, and usual inclusion in forest inventories and measurements of structural complexity. Snags greater than $1.3 \mathrm{~m}$ in height (i.e. a dbh was measurable) were counted in each plot and their $\mathrm{dbh}$ and height were recorded in order to calculate average and standard deviation of snag size as well as dead basal area, which has been used in other structural complexity indices (Van den Meersschaut and Vandekerkhove, 1998; McElhinny et al., 2006).

Within each plot CWD, which was considered to be those with diameters $>7.5$ cm (Stevens, 1997; CFIC, 2004), was sampled using a line intercept method (Van Wagner, 1964; Kirby et al., 1998; CFIC, 2004). This approach was adopted in order to provide measures of the number of pieces of debris in the plots as well as the estimated volume. Van Wagner's (1964) method, which is a commonly used method for estimating woody debris volume (e.g. Ter-Mikaelien et al., 2008), was used:

$$
V=\frac{\pi \sum d^{2}}{8 L}
$$

where $V$ is the volume of CWD per unit area $\left(\mathrm{m}^{3} / \mathrm{ha}\right), d$ is the CWD diameter $(\mathrm{cm})$ where it intersected the transect line, and $L$ is the length $(\mathrm{m})$ of the transect. Sampling was carried out along the diagonals of the $20 \mathrm{~m} \times 20 \mathrm{~m}$ plots (Figure 7), resulting in a total transect length of $56 \mathrm{~m}$. Table 1 provides a summary of all of the variables measured and derived from the field plots. 
Table 1. Summary of field variables measured and derived from field plots representing the various aspects of structural complexity in the forest.

\begin{tabular}{|c|c|c|}
\hline Structural Attributes & $\begin{array}{l}\text { Measurements and } \\
\text { Sampling Methods }\end{array}$ & Derived Variables \\
\hline $\begin{array}{l}\text { Overstorey Trees } \\
\text { (>10 cm dbh) }\end{array}$ & $\begin{array}{l}\text { dbh }(\mathrm{cm}) \text {; height }(\mathrm{m}) \text {; crown } \\
\text { diameter }(\mathrm{m})\end{array}$ & $\begin{array}{l}\text { \# of trees; \# of large trees } \\
(>35 \mathrm{~cm} d b h) ; \bar{x} \text { and S.D. } \\
\text { for each plot; live basal } \\
\text { area }\left(\mathrm{m}^{2} / \mathrm{ha}\right)\end{array}$ \\
\hline Tree Spacing & $\begin{array}{l}\text { Nearest neighbour (NN) } \\
\text { distance }(\mathrm{m}) \text { between } \\
\text { overstorey trees }\end{array}$ & $\bar{x}$ for each plot \\
\hline $\begin{array}{l}\text { Canopy Openness and } \\
\text { LAI }\end{array}$ & $\begin{array}{l}\text { Hemispherical photographs } \\
\text { (camera height: } 1 \mathrm{~m} \text { and } 6 \\
\mathrm{~m} \text { ) }\end{array}$ & $\begin{array}{l}\text { Openness }\left(0^{\circ}-60^{\circ}\right)(\%) \\
\text { and } L A I\left(55^{\circ}-60^{\circ}\right) \\
\text { derived for each height }\end{array}$ \\
\hline $\begin{array}{l}\text { Ground and Understorey } \\
\text { Vegetation }\end{array}$ & $\begin{array}{l}5 \text { height classes, along } \\
\text { diagonals every } 50 \mathrm{~cm}:< \\
10 \mathrm{~cm}, 11-50 \mathrm{~cm}, 51 \mathrm{~cm} \\
-1 \mathrm{~m}, 1-2 \mathrm{~m} \text {, and }>2 \mathrm{~m}\end{array}$ & $\begin{array}{l}\% \text { of plot covered by } \\
\text { vegetation in each class }\end{array}$ \\
\hline Snags & $\begin{array}{l}\text { Presence; dbh }(\mathrm{cm}) \text {; height } \\
(\mathrm{m})\end{array}$ & $\begin{array}{l}\text { \# of snags; } \bar{x} \text { and S.D. for } \\
\text { each plot; dead basal area } \\
\left(\mathrm{m}^{2} / \mathrm{ha}\right)\end{array}$ \\
\hline $\begin{array}{l}\text { Coarse Woody Debris } \\
\text { (> 7.5 cm diameter) }\end{array}$ & $\begin{array}{l}\text { Diameter }(\mathrm{cm}) \text { of each } \\
\text { piece of dead wood where } \\
\text { it crossed one of the } \\
\text { diagonals }\end{array}$ & $\begin{array}{l}\text { \# of pieces; estimated } \\
\text { volume }\left(\mathrm{m}^{3} / \mathrm{ha}\right) \text { (Van } \\
\text { Wagner, 1964) }\end{array}$ \\
\hline
\end{tabular}

$\overline{\bar{x}}=$ average calculated for all trees or snags in the plot

S.D. = standard deviation calculated for all trees or snags in the plot 


\subsubsection{Explanatory / Predictor Variables}

A set of variables was extracted from the imagery based on both the results from other studies of empirical modeling of forest structural attributes (cited under each category of image measure described below) and according to their hypothesized contribution to differentiating areas of different structural complexity in the forest. This research included a comprehensive suite of image variables in order to attempt to test which were most useful for modelling forest structural complexity. Although image brightness variations result from variations in forest spectral reflectance, which are influenced by forest structure, the terms 'explanatory / predictor' are used throughout the thesis since the goal was to predict forest structure given a set of variables extracted from the imagery.

While the field plots were originally set up as $20 \mathrm{~m}$ x $20 \mathrm{~m}$ squares, they were not perfectly square. More importantly, since magnetic declination $\left(13^{\circ} 40^{\prime}\right.$ West $)$ was not taken into account when setting up the plots, they were all tilted and did not match up well with pixel rows and columns in the imagery. Accordingly, for easier processing the plots were treated as circles with $11.3 \mathrm{~m}$ radii in order to keep the area the same $(\sim 400$ $\mathrm{m}^{2}$ ) while still using the original plot centre location. Any mismatch caused by the different plot shape was within the associated image and GPS error $(<3 \mathrm{~m})$.

The variables extracted from the imagery represented: (1) spectral information, including radiometric fractions at the pixel and sub-pixel level, (2) spatial information calculated from both the grey-level co-occurrence matrix (GLCM) and semivariograms, and (3) object-based information providing information on canopy and shadow objects. 
Spectral and spatial information were extracted for the entire plot as well as from withincrowns and within-shadows separately. Details of each are given in the following sections.

A wide variety of variables was tested in order to attempt to represent different aspects of horizontal and vertical structural complexity as it may have manifested itself in the airborne imagery through radiance variations. In general, in terms of the variables extracted from the imagery, areas of the forest with higher complexity were expected to have lower reflectance compared to areas with lower complexity as a result of the presence of canopy gaps, representing heterogeneity among the overstorey trees. As well, the presence of dead wood in the forest, which was associated with greater structural complexity, was expected to be detected through higher reflectance in the red band. Areas of higher complexity were also expected to be associated with greater spatial variability in image brightness, at the plot and sub-plot level, due to the greater presence of gaps / shadows in the overstorey. It was also hypothesized that more complex areas would have a greater number of canopy objects, and potentially a greater variation in overstorey tree sizes, which could potentially be approximated through tree delineation methods.

\subsubsection{Image Spectral Variables}

The average image brightness of each spectral band, as well as NDVI, was extracted for each field plot. It was expected that plot average image brightness in more complex canopies with a greater mix of crowns and shadows would be lower than in more uniform closed canopies. Similar to Seed and King (2003), unsupervised 
ISODATA clustering was used to classify the imagery into thirty clusters that were then aggregated to create a map of forest canopy, shadow, and exposed wood. The percentage of each plot covered by these three components was calculated and the average brightness of each spectral band was extracted for each plot. Additionally, constrained linear spectral unmixing was used, with endmembers manually extracted from the imagery, in order to extract sub-pixel fractions of sunlit canopy, shadow, and exposed wood. Finally, the average brightness of each was extracted for each plot (Holben and Shimabukuro, 1993; Peddle et al., 1999; Lévesque and King, 2003; Pasher et al., 2007).

\subsubsection{Image Spatial Variables}

As first-order texture measures, the standard deviations of plot image brightness were extracted for each spectral band as well as from the unmixed fraction images. Second-order texture measures were extracted from the first Principal Component of the three spectral bands $(\mathrm{PCl}=94.9 \%$ of total variance). Five commonly used GLCM texture measures (Contrast, Entropy, ASM, Correlation, and Homogeneity) (Haralick et al., 1973), which have been shown to be useful for modelling forest structure attributes (Franklin et al., 2000; Cosmopoulos and King, 2004; Kayitakire et al., 2006) were extracted for each field plot. They were calculated using both a $5 \times 5$ pixel $(1 \mathrm{~m} \mathrm{x} 1 \mathrm{~m})$ and $25 \times 25$ pixel $(5 \mathrm{~m} \times 5 \mathrm{~m})$ moving window in all sample directions (i.e. omnidirectional). The statistics extracted for these moving window measures were taken from all pixels in the plots except the very edge pixels which contained information from outside of the plot. These window sizes were selected following tests showing their ability to differentiate between plots of high and low complexity. The smaller window 
size was used to represent intra-object variation (within-crowns and shadows), while the large window size represented inter-object variation (crowns / canopy objects vs. between crown shadows).

As for the first order standard deviation described above, the GLCM textures were expected to reflect increasing variability with increasing forest structural complexity. However, this turned out to be inversed. Close analysis showed that for structurally complex field plots with large openings in the overstorey canopy, which manifested themselves as large deep shadows in the imagery, resulted in smoother texture measures. This was because both window sizes often included only shadow or a significant portion of nearly constant shadow brightness. As a consequence of this, combined with the fact that full plot information was somewhat bimodal, texture measures were extracted separately from the classified canopy and shadow areas (following the ISODATA clustering) in each plot using the $5 \times 5$ pixel window, which were thought to be more useful than the full plot-based information.

Semivariograms were calculated for each spectral band individually within each plot in order to extract measures that have shown strong relations to forest structure (St. Onge and Cavayas, 1997; Treitz and Howarth, 2000; Lévesque and King, 2003; Zawadzki et al., 2005). The range of the semivariogram, which indicated the distance at which pixels are no longer spatially correlated, and is often related to the size or scale of dominant objects in the imagery, was calculated (Curran, 1988; Johansen et al., 2007). As well, the sill, which indicated the total structurally dependent variance in the data, was calculated as a measure of the complexity of the image data (Johansen et al., 2007). 


\subsubsection{Image-Object Variables}

Finally, relating directly to field measurements specifically, crown sizes, horizontal tree distribution, and canopy openness, tree crowns and shadows were isolated in the imagery as individual objects. Tests of automated individual crown detection and delineation using an algorithm from Pouliot et al. (2005) were not successful in this forest where crowns overlap significantly (V. Torontow and D. King, unpublished data). However, the algorithm was very good at separating canopy objects (representing branch groups, individual crowns or multiple crowns) from shadows. These canopy objects were extracted and the remaining shadow pixels were converted to vector polygons with contiguous pixels grouped as individual objects. The number and size distribution of the canopy and shadow objects were measured for each plot. Further, a simple measure of shape complexity representing the deviation from circularity (simplest shape), was calculated based on the perimeter : area ratio (Patton, 1975; Ewers et al., 2007). Shape complexity, particularly of canopy objects, was investigated since it was hypothesized that trees missing parts of their crowns, an attribute typically associated with higher structural complexity, might be differentiated from more circular shaped whole crowns.

\subsubsection{Topographic Variables}

The relative position in the landscape can have an impact on forest structural conditions in terms of susceptibility to disturbance (e.g. wind exposure) (Turner and Dale, 1998; DeGayner et al., 2005), productivity (e.g. exposure to sunlight) (Waring, 2000; Xu et al., 2008) and regeneration following disturbance (de Lima and de Moura, 2008), a result topographic information was included in the modelling exercise. 
A $10 \mathrm{~m}$ resolution digital elevation model (DEM) was created from elevation points that had been generated from $25 \mathrm{~cm}$ resolution leaf-off orthophotos $(\mathrm{NCC}, 1998)$. The elevation points were interpolated using tension spline interpolation, which fit through the input data and resulted in a smooth surface. The surface was evaluated using forty-two independent GPS test points and found to have a vertical RMSE of $7.52 \mathrm{~m}$ (Bemrose, 2006). Taking into account taking into account a $\mathrm{z}$ accuracy of $3 \mathrm{~m}$ for the test points, the total vertical RMSE of the DEM was calculated to be $5.72 \mathrm{~m}$. Slope and aspect maps were derived from the DEM using standard GIS algorithms (ArcGIS 9.2; ESRI, 2008), and northness (sine of aspect) and eastness (cosine of aspect) were calculated and used instead of aspect itself, which is a circular variable (Guisan et al., 1999; Lassueur et al., 2006).

A solar illumination (SI) image was also created using the sun's position at the time of image acquisition (solar zenith angle $=33.7^{\circ}$ and solar azimuth angle $=190.2^{\circ}$ ) following the equation provided by Riano et al. (2003), as follows:

$$
S I=\cos \theta_{p} \cos \theta_{z}+\sin \theta_{p} \sin \theta_{z} \cos \left(\phi_{a}-\phi_{o}\right) \quad \text { [2] }
$$

where $\theta_{p}$ is the slope angle (i.e. slope or terrain), $\theta_{z}$ is the solar zenith angle, $\phi_{a}$ is the solar azimuth angle, and $\phi_{o}$ is the surface aspect angle. Since the image acquisition was close to solar noon, SI was hypothesized to be well correlated with the total illumination throughout the growing season. Given this, the variable had potential for providing some explanation of the variance of forest structure related to productivity. Table 2 provides a 
summary of all of the different variables used as potential explanatory / predictor variables for modelling structural complexity.

\subsubsection{Modelling Structural Complexity Using Redundancy Analysis}

The multivariate nature of both forest structural complexity, as seen by the many measures needed to quantify the various aspects of a forest's structure, and remotelysensed imagery lends itself well to multivariate statistical techniques designed to explore relationships between multiple predictors and response variables. While researchers such as Wunderle et al. (2007) reduced the dimensionality of a set of field data to a single variable through PCA in order to carry out modelling, the statistical methods exist in order to directly examine relations between multiple dependent variables and multiple independent variables all at once. PCA is the most commonly used method for reducing the dimensionality of multivariate data while preserving as much of the relevant information as possible (Jolliffe, 2002). However, in simple environments such as that investigated by Wunderle et al. (2007) where a single component representing $79 \%$ of the total variance can be created, in more complex environments several components are often necessary to capture the variance in the data. For example, Cosmopoulos and King (2004) reduced their field variables from nine structural attributes down to three components representing $\sim 81 \%$ of the original variance, and similarly reduced their image variables down to four components also representing $\sim 81 \%$ of the original variance. Several conflicting methods exist for selecting the significant components that should be retained following a PCA, also known as "stopping rules". These include strategies such as selecting those components with eigenvalues greater than 1.0 
Table 2. Summary of image variables extracted from multispectral airborne imagery to be used as predictors for modelling structural complexity.

Variable Type

Spectral

Pixel level

Subpixel

Spatial

Object-based

\section{Canopy and Shadow objects}

\section{Variables Extracted / Derived}

- $\%$ of plot classified (ISODATA unsupervised clustering) as vegetation, shadow, and exposed wood.

- $\bar{x}$ and S.D. of NIR, R, G, and NDVI within each plot.

- Variables calculated for full plot as well as for the vegetation and shadow components of the plot individually.

- $\bar{x}$ and S.D. of fraction of sunlit crown, shadows, and exposed wood derived through linear spectral unmixing.

- $\bar{x}$ and S.D. of 5 texture measures calculated from the grey level co-occurrence matrix (GLCM) (Haralick et al., 1973) using $5 \times 5$ window.

- Variables calculated for full plot as well as for the vegetation and shadow components of the plot individually.

- Range and sill from theoretical semivariogram of each spectral band.

- \#, $\bar{x}$ and S.D. of size of canopy objects in each plot (using tree delineation algorithm (Pouliot et al., 2005)) and area of shadow objects.

- $\bar{x}$ and S.D. of shape complexity within each plot (deviation from circularity: Patton, 1975)

- Elevation, slope, and aspect.

- Solar illumination calculated at image acquisition time (Riano et al., 2003).

$\overline{\mathrm{x}}=$ average calculated for all pixels in the sample

S.D. = standard deviation calculated for all pixels in the sample 
(Kaiser, 1960), examining scree plots (Horn, 1965) as well as the "broken-stick method" (Frontier, 1976). As a result, it is often difficult to decide what information should be retained for further analysis, and thus too many components with little if any meaning are often retained (Jackson, 1993). Datasets with high dimensionality are therefore often difficult to reduce and interpret using a PCA.

Building on the previously discussed research by Olthof and King (2000), Sampson et al. (2001), Cosmopoulos and King (2004), and de la Cueva (2008), this research used a Redundancy Analysis (RDA) (Rao, 1964; Stewart and Love, 1968) for modelling structural complexity. Modelling structural complexity using a combined approach of PCA, CCA and multiple regression, as was carried out by Cosmpopoulos and King (2004), requires extensive interpretation at several stages throughout the analysis and is not a direct method for modelling multivariate data. RDA can actually be thought of as a PCA of multivariate regression predicted values (Stewart and Love, 1968; Legendre and Legendre, 1998), and is therefore a more direct method for modelling multivariate data in cases such as this. RDA is in fact the proper statistical tool for carrying out this type of analysis and provides outputs designed specifically for dealing with this type of data. Another advantage of RDA over other methods is its ability to simultaneously and directly analyze multiple dimensions of forest structure (i.e. multiple canonical axes) that may exist independently from one another.

RDA provides a method for describing relations between multiple explanatory / predictor variables (in this case image and topographic variables) and multiple response variables (structural measurements taken in the forest) (Legendre and Legendre, 1998; ter 
Braak and Šmilauer, 2002). RDA was designed to express how much of the variance in one set of variables can be explained by the other, in other words describing the redundant information between two data sets (Legendre and Legendre, 1998). The method was originally developed in order to examine effects of multiple environmental factors on many species simultaneously, and is one of many multivariate techniques, known as constrained ordination, that order or arrange high dimensionality data along one or more simplified gradient axes (Pielou, 1984; ter Braak, 1987; Palmer, 2008). While the RDA methodology is applicable to many different fields, it continues to be used by researchers mainly to explain species abundance or diversity using environmental variables (e.g. Comor et al., 2008; Sanchez et al., 2008; Yamaura et al., 2008).

In a simplified manner, an RDA is carried out by calculating multiple linear regressions for each response variable (y) individually (in this case the field variables), followed by a single PCA of the fitted / predicted response variables (y'). The resultant components, or canonical axes, which are uncorrelated and each represent decreasing portions of the original variance in the set of response variables, can then be interpreted in terms of their relation to the original field variables (similar to examining factor loadings in a PCA) and the explanatory / predictor variables that were included in the analysis (Legendre and Legendre, 1998).

For this analysis, while a unimodal response between the explanatory and response variables could be hypothesized, a linear response was assumed following the necessary tests for unimodality described in ter Braak and Šmilauer (2002). This test 
examined the "length of gradient" statistic in order to make sure that it was below a standard threshold of 4 standard deviations. This examination was based on the assumption that, if it was greater than this threshold, it would imply that the dataset showed too much deviation from a linear model (Lepš and Šmilauer, 2003). The statistics was found to be 3 , well below the accepted threshold.

It could be hypothesized that certain field variables, such as canopy openness for example, would exhibit unimodal relationships with certain image variables (e.g. image texture measures). Both an extremely open and closed canopy (i.e. approaching $0 \%$ or approaching $100 \%$ canopy cover) will result in homogeneity in the imagery, while heterogeneity in the imagery would be seen with intermediate closure in the canopy. The canopy openness (based on hemispherical photographs collected at $6 \mathrm{~m}$ ) ranged from 3.3 $\%$ to $45.5 \%(\overline{\mathrm{x}}=15.6 \%$, S.D. $=8.8 \%)$, which matched previous work in the forest by Bemrose (2006), who measured gap fractions, or percentage of open sky visible, ranging from $4.6 \%$ to $54.0 \%(\overline{\mathrm{x}}=17.6 \%$, S.D. $=11.1 \%)$. This is clear evidence that only half of the full distribution of potential levels of canopy openness (i.e. $0-100 \%$ ) existed in the field plots, which were representative of the study area forest. As a result only half of the response curve existed in this data and therefore the data were approximated using a linear relationship.

Rather than creating an RDA model that was solely dependent on the set of input data, a bootstrapping procedure was implemented to provide more robust results. A twostage RDA analysis was designed, which first used bootstrapping for variable selection and then a second stage of bootstrapping for model validation by providing measures of 
variance within the models. The procedure was programmed in Matlab (included in Appendix 2) incorporating existing RDA code from Peres-Neto et al. (2006) as well as general statistical procedures from Jones (2008) (R. Proulx, pers. comm., 2008). Fivehundred iterations of the RDA using forward stepwise selection were run. Each iteration involved a random selection of forty out of the fifty field plots. This stage of the analysis produced five-hundred potentially unique models, which were then individually explored and validated within the second bootstrapping stage.

Each of these five-hundred models was run through an RDA, this time not using stepwise selection, but instead entering only those variables that were entered in the first stage for that model. This second stage was also run for five-hundred iterations, selecting forty out of fifty plots each time to produce a model. Statistics were calculated and recorded for each iteration, permitting summary statistics to be collected for each RDA model, including the average adjusted $\mathrm{R}^{2}$ and the average Akaike information criterion (AIC) (Akaike, 1974), which is often used for model selection.

As well, each RDA model was tested using the ten plots that had been withheld at that iteration, which were therefore independent of the model. The predicted first RDA axis $\left(\mathrm{RDA}_{\text {Predicted }}\right)$ was calculated by applying the $\mathrm{RDA}$ model to the set of image variables of the ten validation plots. $\mathrm{RDA} \mathrm{Aredicted}_{\text {represented the predicted canonical axis }}$ produced from a linear combination of predictor (i.e. image) variables. This variable was then directly compared to the corresponding variable calculated using the observed information ( $\left.\mathrm{RDA}_{\mathrm{Observed}}\right)$, in other words the actual twenty-four field variables, measured in each of the ten plots. Therefore, similar to validating multiple regression models, only 
with multiple dependent variables instead of a single one, a predicted y variable (RDA $\left.A_{\text {Predicted }}\right)$ and an observed y variable (RDA Observed $)$ were calculated. Since the RDA produced canonical axes that were in a different projected space from the original data, the observed field variables were first transformed into the equivalent projected space as the modeled canonical axes using a transformation matrix ( $U$ Matrix (Legendre and Legendre, 1998; R. Proulx, pers. comm., 2008)). The correlation (referred to from this point on as "r-validate") and the RMS error (\% RMSE) between RDA $A_{\text {Predicted }}$ and RDA $_{\text {Observed }}$ were then calculated and the average values were derived (over five-hundred iterations) for each model.

The summary validation statistics were individually ranked from best to worst, and the top models were selected based on the best overall ranking (i.e. highest Adjusted $\mathrm{R}^{2}$, lowest AIC, fewest variables, lowest RMSE, and highest r-validate). Further manual exploration of the models resulted in a single selected model. This final RDA model was processed using CANOCO 4.5 (ter Braak and Šmilauer, 2002), which is a specialized software package designed specifically for carrying out ordination analyses, in order to obtain the desired numerical and graphical outputs. Field variable scores ("species scores" in the software terminology) and "biplot scores of environmental variables" (predictor variable scores) were examined in order to both interpret the model as a whole and understand the influence of individual predictor variables.

A triplot was also created for visualization of the relationships described by the model. Triplots (or biplots) are commonly used for interpreting results of RDA among other ordination techniques (e.g. Jelaska et al., 2006; Comor et al., 2008; de la Cueva, 
2008) by aiding the interpretation of relations between variables (both field and predictor) as well as displaying the position of different field plots on the RDA axes.

Finally, the regression / canonical coefficients for the model were retained in order to construct a predictive model for forest structural complexity mapping. A process map is shown in Figure 8. This summarizes the entire RDA modelling process, including mapping, validation, and scalability testing, which will be discussed in the following sections.

\subsubsection{Mapping Structural Complexity}

RDA analysis identifies a linear combination of predictor variables (image variables) that best explains the strongest gradient(s) within the set of field variables (structural attributes) (Legendre and Legendre, 1998). The CANOCO output for the final RDA model included a matrix of regression / canonical coefficients for standardized variables (ter Braak and Šmilauer, 2002). The coefficients for the $1^{\text {st }}$ RDA axis (the only significant axis) were applied to standardized spatial layers representing each of the image and topographic variables that were included as predictors in the model. This RDA

axis, based on its relationships with the different field variables, was defined as the Structural Complexity Index (SCI). The linear combination of these coefficients, multiplied by the spatial layers, provided a continuous map of the predicted SCI. 
MODEL INPUTS:

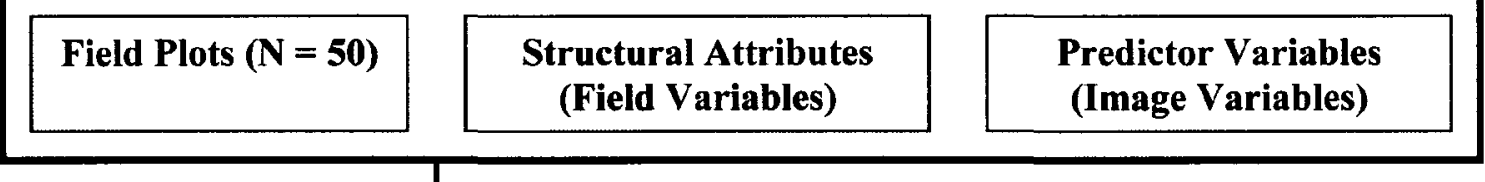

STAGE 1 BOOTSTRAPPING:

Variable Selection - 500 Iterations:

Random 40 out of 50 field plots selected

RDA model using forward stepwise selection procedure

STAGE 2 BOOTSTRAPPING:

Validation - 500 Iterations:

RDA using only the predictor variables selected by the stepwise selection
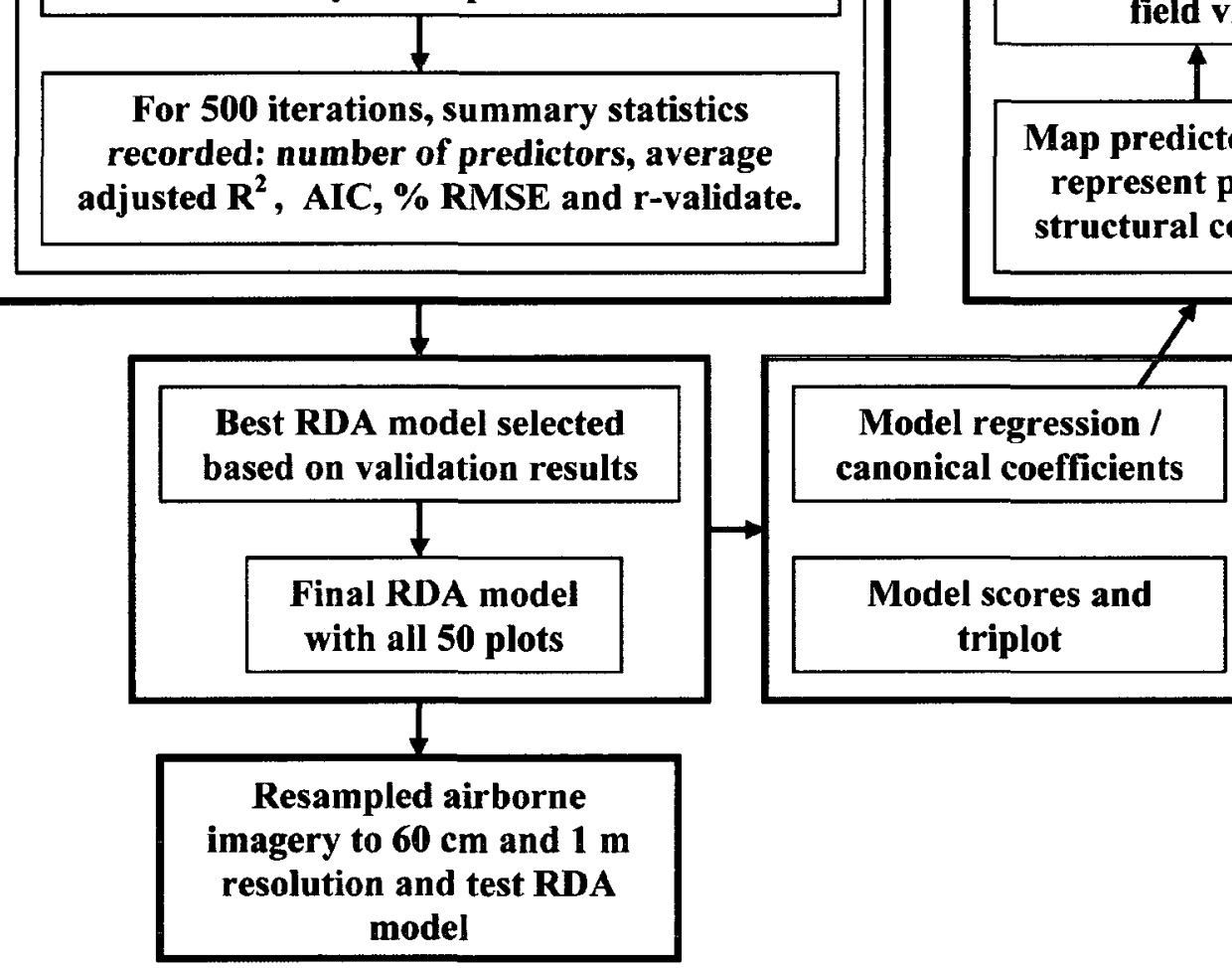

Figure 8. RDA modelling process showing two-stage bootstrapping, final model selection, mapping and validation, as well as multiscale model testing. 


\subsubsection{Structural Complexity Map Validation}

The structural complexity model was already considered to be fully validated based on the samples excluded during the bootstrapping process, which is a commonly used method for validating biophysical models (e.g. Cohen et al., 2003; Olthof et al., 2004). This bootstrapping approach simulated a field validation campaign that would have required the establishment of a new set of field plots and the measurement of all field variables within those plots in order to calculate the SCI independently of the model. This process provided validation of the continuous gradient of structural complexity, rather than splitting the SCI into classes.

In addition to this process, a further validation of the extreme conditions found in the SCI map was carried out for the simple reason that the extreme conditions are potentially of more interest in the field from a amangement perspective. Areas of predicted relatively very high and relatively very low complexity were manually identified from the SCI map. With the map classified using a standard deviation classification, areas classified as having predicted SCI greater than two standard deviations from the map's mean value were selected. These areas were visited in the field (with the aid of a GPS) in a random order; in a sense eliminating any a priori knowledge of the predicted level of structural complexity. These locations were spatially independent of the field plots and were in fact in areas of the forest that had never been previously visited by the researchers, removing any a priori knowledge of conditions in the field that may have biased their selection as validation sites. Individual structural attributes were visually assessed within the visited areas using a similar approach that 
was used for initial plot establishment (described in Section 4.3.1). Following discussions, two evaluators arrived at a single conclusion with respect to the approximate level of structural complexity found in the forest. Additionally, the area surrounding each location (approximately a $20 \mathrm{~m}$ buffer) was examined to determine if the selected locations were significantly different from their surroundings.

Seventeen relatively high complexity areas $\left(\overline{\mathrm{x}}\right.$ area $=1743 \mathrm{~m}^{2}$, range $=450 \mathrm{~m}^{2}-$ $4311 \mathrm{~m}^{2}$ for the selected areas) and eight relatively low complexity regions $(\overrightarrow{\mathrm{x}}$ area $=$ $5692 \mathrm{~m}^{2}$, range $=1108 \mathrm{~m}^{2}-11532 \mathrm{~m}^{2}$ ) were visited as part of the field validation. The areas were visually assessed and documented in the field and, following the field visits, it was decided that regions would be validated using two schemes. Initially the regions were looked at in terms of being correctly or incorrectly classified, however they were also separately assessed using a simple point system. Here, correctly classified ( 1 pt) indicated that the full region was correctly classified, partially correctly classified $(0.5$ pts) indicated that while much of the area $(>50 \%)$ was correctly classified there were areas within the region that were not and, finally, incorrectly classified areas received no points.

\subsubsection{Testing the Potential Scalability of the Methods}

The high spatial resolution of the airborne imagery used in this study was selected based on previous experience and expected contributions from spatial and object information in the structural complexity model. However, the collection of airborne imagery requires far greater planning and processing compared with satellite imagery. Currently available satellite sensors include Quickbird $(\sim 60 \mathrm{~cm}$ panchromatic, $\sim 2.4 \mathrm{~m}$ 
multispectral (Digital Globe, 2008)) and GeoEye-1 $(\sim 41 \mathrm{~cm}$ panchromatic, $\sim 1.65 \mathrm{~m}$ multispectral (GeoEye, 2008)), and even higher resolution sensors are planned for the near future, including the GeoEye-2 satellite with $25 \mathrm{~cm}$ panchromatic imagery (GeoEye, 2008).

The fusion of panchromatic and multispectral imagery can currently provide submeter spatial information. Therefore one of the objectives of this study was to evaluate multivariate image-based forest structural complexity modelling and mapping at multiple scales. This was not meant as a comprehensive analysis of model scaling and scale dependence of variables, which is currently being examined in other ongoing research using Quickbird imagery (D. King, unpublished data), but rather an initial investigation into the scalability of the methods used in this research.

In order to accomplish this, following RDA modelling, the airborne imagery was resampled from $20 \mathrm{~cm}$ to $60 \mathrm{~cm}$ and then to $1 \mathrm{~m}$ spatial resolution using a $3 \times 3$ and $5 \times 5$ Gaussian filter, respectively, which approximated the point-spread function of a sensor with coarser resolution (following the methods presented in Olthof et al., 2005). A slightly reduced set of image predictor variables $(n=73)$ was extracted from the two new lower resolution images. Some variables that were highly correlated with others that were much easier to extract were not included in this analysis. For example the object-based area of shadows within the plots was very correlated with the percent shadow in each plot calculated using a simple unsupervised clustering procedure. As well, the range and sill calculated from semivariograms from the red and green bands were very highly correlated with corresponding variables calculated from the NIR band. 
A full RDA analysis was carried out for each of the two new datasets following the methods presented in Section 4.3.4. This included bootstrapping for predictor variable selection as well as model validation. In each case, the top models produced, based on the validation statistics calculated within the RDA process, were tested by running an RDA within CANOCO 4.5, which provided full results of the analysis, and assessed for any variance inflation caused by collinearity between predictor variables. In each case, the best model was selected and, following the mapping procedures presented in Section 4.3.5, two new maps of predicted SCI were produced. The models and maps produced using the coarser resolution datasets were compared to those created using the original 20 $\mathrm{cm}$ imagery. Finally, the maps produced at the coarser resolutions were validated by comparing them to the original SCI map, as well as using the field validation areas and scoring system originally used (Section 4.3.6).

\subsection{Modelling and Mapping Dead Wood}

Forest dead wood, which is only one aspect of forest structural complexity, has been directly linked to habitat and biodiversity levels and is therefore considered a useful indicator. This next section describes the methods used for indirectly modelling and directly detecting dead wood, as well as mapping the spatial distribution of dead wood across the study area using remote sensing.

\subsubsection{Initial Field Exploration}

An initial reconnaissance trip was made to the forest in order to better understand what types of snags were present in the area, to attempt to hypothesize which snags 
should be detectable using the airborne imagery, and to try to understand the issues or potential problems that might be faced in this type of analysis. It became evident that snags that were snapped off well below the height of overstorey tree crowns would not be detectable since they would be located in shadows within the imagery. Snags with full or partial crowns intact, as well as snags without any branches that were close to the height of the surrounding overstorey crowns, were considered to be potential targets (examples shown in Figure 9). It was obvious that coarse woody debris (CWD) was not directly detectable, other than cases where large intact trunks lay beneath large gaps in the canopy, and therefore CWD was only investigated for potential indirect modelling purposes.

\subsubsection{Remotely-Sensed Imagery}

The imagery used, along with some of the derived variables, was the same as was used for the development of the structural complexity index as previously described. The airborne imagery was acquired with a $20 \mathrm{~cm}$ resolution, which theoretically permitted snags or individual dead branches of approximately $20 \mathrm{~cm}$ in diameter to be detected. 

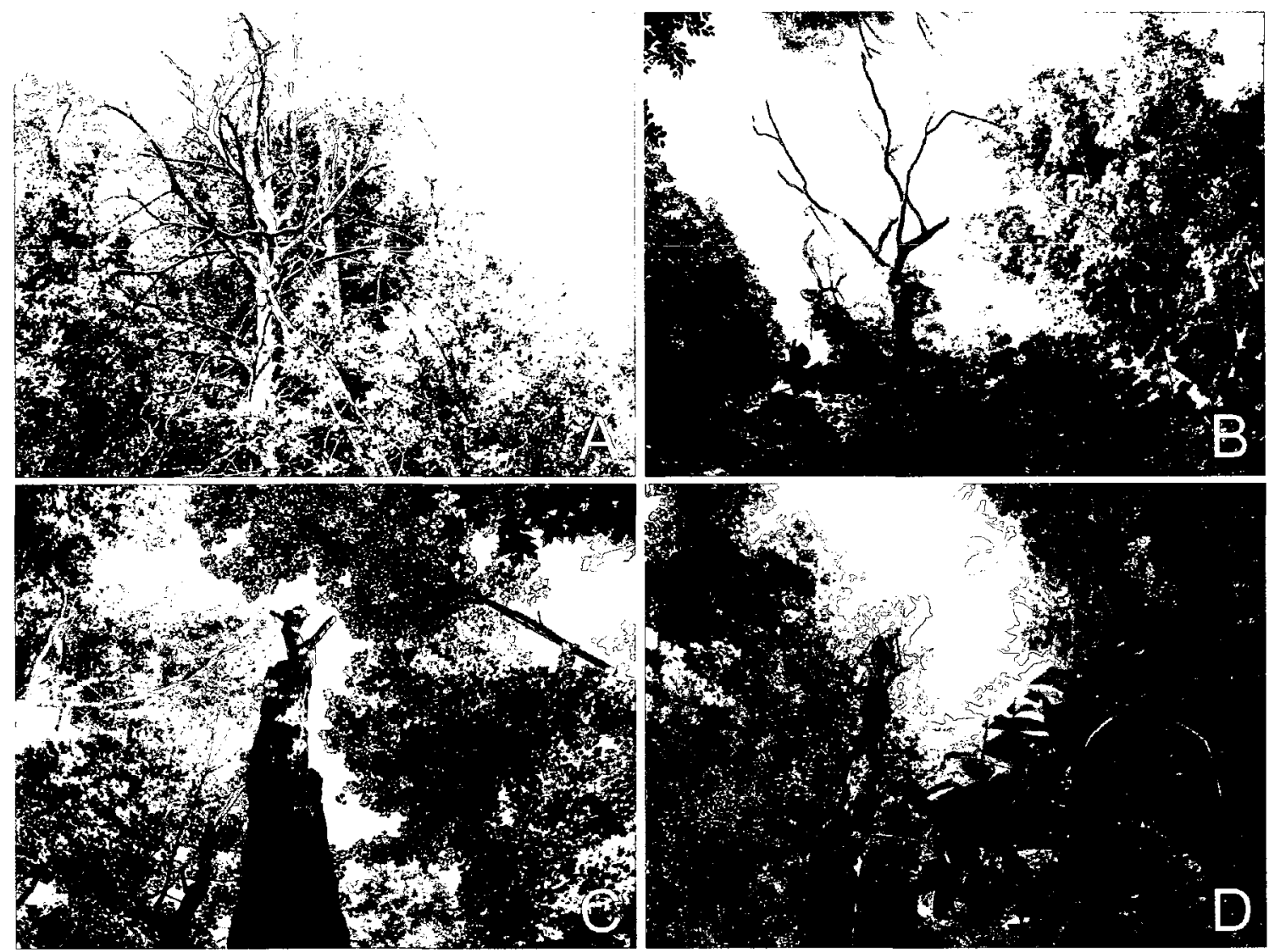

Figure 9. Examples of snags thought to be suitable for detecting and mapping, including those with fairly intact crowns (A and B) and those with no branches remaining (C and $D$ ), but found in canopy gaps and / or of heights similar to the surrounding live overstorey trees.

\subsubsection{Direct Detection of Dead Wood in Imagery}

The multispectral imagery allowed clear manual identification of much of the dead wood in the upper canopy and some dead wood lower in the canopy or on the ground in large gaps. With respect to directly detecting dead wood in the imagery, the more general term "dead wood" will hereafter be used rather than snags, which was felt to be too specific and not representative of the objects that would possibly be detected. 
Although in some rare cases CWD was thought to be detectable in the imagery, for the most part the objects that were investigated in this part of the analysis were snags, partially dead crowns and single dead branches within live crowns, all being indistinguishable from each other in the imagery. The partially dead crowns and single dead branches were deemed to indicate varying degrees of tree decline that would most likely produce CWD or snags within the next several years.

In the multispectral imagery, dead wood was visually distinguishable from surrounding live vegetation based mainly on its higher reflectance in the red band and its lower reflectance in the NIR band (Jensen, 2006). For the relatively small area of image coverage of this study, manual digitizing could have been used to delineate occurrences of dead wood and produce a map of its spatial distribution. However, some dead wood that was partially obscured by shadow or foliage was not easily identified. Further, and more importantly, the intention of this research was to investigate more automated means of dead wood detection and mapping that could be applied more efficiently than manual interpretation. Three methods were used in combination in order to exploit different types of information in the spectral reflectance characteristics of dead wood compared to the surrounding foliage and shadow. Using the methods in combination was expected to increased the confidence in the detection of the individual objects.

\subsubsection{ISODATA Unsupervised Clustering}

Initially, an ISODATA unsupervised clustering algorithm was used. It is a common technique to automatically group pixels within an image into clusters using an iterative process based solely on the spectral properties of individual pixels (Jensen, 
2006). Clustering techniques such as this were originally developed to be used at coarser scales for landcover mapping, but in this research it was used with high resolution imagery to create a thematic map of exposed wood isolated from the surrounding vegetation and from within-crown shadows. This technique was used to create a dead wood cluster in a manner similar to that used by Kelly et al. (2004). Twenty clusters were arbitrarily generated and manually aggregated through comparison with the CIR imagery in order to produce a map of three classes representing exposed wood, live vegetation and canopy shadows. This map was then converted to a binary map representing the detected exposed wood (1) and everything else (0).

\subsubsection{Sub-pixel Wood Fractions using Unmixing}

As the ISODATA algorithm is a hard classifier, transition pixels between classes (e.g. pixels at edges between exposed wood and surrounding vegetation) are often confused. This is a result of their mixed radiance from more than one cover type. These pixels are assigned to one class based on a given rule such as the minimum distance of the pixel to each class mean (as in the ISODATA algorithm) or the probability of belonging to each class (as in the maximum likelihood classifier). However this can often result in a poor representation of reality (Settle and Drake, 1993; Oki et al., 2002). In addition, wood that was smaller than the pixel size and in shadow may not have produced enough radiance to result in a hard classification as wood but may instead have been assigned to the shadow class. As a result, soft classification techniques, such as linear spectral unmixing, have been developed that retain more information from within mixed

pixels (Bastin, 1997). Since the reflectance of a pixel is often made up of more than one 
type of object, or scene component, spectral unmixing can be used to decompose the mixed spectra in order to determine sub-pixel proportions of each (Peddle et al., 2001). Given the "linear" nature of the procedure, the reflectance values associated with a mixed pixel are assumed to be linear combinations of the reflectance of the constituent classes that are within the bounds of that pixel (Klein Gebbinck, 1998; Brown, 2001). A constrained least squares linear unmixing procedure was used in order to produce images representing the fractional coverage of each class (Holben and Shimabukuro, 1993; Peddle et al., 2001).

Spectral unmixing relies on the selection of endmembers representing pure pixels of each of the desired classes to be used as training data. Endmember data were manually selected by delineating samples of the purest pixels of sunlit crowns (i.e. live vegetation), shadows, and exposed wood across the imagery (Pasher et al., 2007). Pixels were unmixed to created fractional coverages, including, of particular interest, a wood fraction image, similar to Lévesque and King (2003) and Pasher et al. (2007), with values for each pixel representing the fractional coverage ranging from 0 to $100 \%$ in terms of the wood content of that pixel. The wood fraction image was then converted to a binary image where all pixels with wood fractions $>50 \%$ (i.e. presumed to be made up of at least 50 $\%$ wood) were classified as wood. This threshold was selected following visual interpretation and iterative testing of the effects of various thresholds on the resultant unmixed layer in order to best isolate those pixels that were in fact wood objects. 


\subsubsection{Object-based Segmentation and Classification}

The final method used to isolate exposed wood within the imagery was a simplified object-based segmentation and classification method carried out using Definiens 5.0. This method works by segmenting, or partitioning, an image into multipixel objects, as opposed to an individual pixel based analysis (as for ISODATA clustering and spectral unmixing) in order to maximize between-object variance and minimize within-object variance (Flanders et al., 2003; Yan et al., 2006). Image segmentation was carried out through iterative testing and visual assessments using the three segmentation parameters: scale, colour (spectral information), and shape (smoothness and compactness), which all contribute to the definition of objects and object sizes (Laliberte et al., 2004). The scale parameter is directly related to the resolution of the imagery and larger values result in larger objects (Benz et al., 2004). A value of 10 was eventually decided upon for this parameter. The colour parameter refers to the relative importance of an object's colour (reflectance) compared to its shape. For exposed wood in the imagery the reflectance was quite homogenous within the class, while the shapes were assumed to be quite heterogeneous. As well, the spectral differences between exposed wood and other objects (i.e. foliage and shadows) were extremely important and consistent as opposed to shape differences between classes, which may have varied significantly. Consequently, after iterative testing (Flanders et al., 2003; Yan et al., 2006), a ratio of $0.9: 0.1$ (Colour : Shape) was used. Given that the shape parameter was set to 0.1 (very low), the compactness / smoothness ratio did not have very much influence on the output objects and was therefore left at the default of 0.5 : 0.5 , with tests showing little change when these values were adjusted. The resulting 
segmented objects were visually examined and found to closely resemble objects that would have been delineated through manual image interpretation.

Following object segmentation, the objects were classified using a nearest neighbour classification (Definiens, 2006; Laliberte et al., 2006; Mallinis et al., 2008) based on spectral similarity to a sample of manually selected segmented wood objects that represented the variety of wood objects in the imagery. While fuzzy membership values, based on the nearest neighbour distance, were assigned to each object (Definiens, 2006), the classification was "hardened" by including all the objects that were assigned to the wood class. Objects with membership values greater than zero were assigned to a final classification layer, which resulted in a layer containing only those objects classified as wood across the imagery. While this selected membership value threshold could have impacted the actual number of detected objects in the classification, visual analysis of the segmented and classified objects showed a clear difference between wood objects and others in the imagery, a result of the reflectance characteristics of wood compared to foliage and shadows.

\subsubsection{Developing a Map of Detected and Delineated Wood Objects}

The binary maps produced by the three dead wood detection methods were filtered to remove "salt-and-pepper" single pixel objects that were classified as wood. These objects were assumed to be either erroneously detected objects as artifacts of the classification methods (similar to Guo et al., 2007), or very small parts of exposed wood that were unfortunately impossible to validate in the field since individual wood objects of that size could not be identified within the canopy from the ground below. The layers 
were then generalized to remove the pixilated appearance and smooth the objects in order to provide more realistic areal estimates. Objects that were separated by a single pixel (i.e. only $20 \mathrm{~cm}$ apart) were combined by application of a single pixel buffer around each object. This had the effect of grouping multiple dead wood objects that were part of a single dead tree crown.

To produce a map with maximum confidence in terms of detected wood across the forest, the three layers were combined using a majority operation on a pixel by pixel basis. In other words, only those pixels that were classified as wood according to at least two of the three methods were assigned to the wood class in the final map. While each method had its advantages and limitations in terms of utilization and results, the intersection of the three methods was thought to provide a much more robust and meaningful result. This approach is similar to the use of multiple, or hybrid classifiers (e.g. Steele, 2000; Kelly et al., 2004; Liu et al., 2004; Guo et al., 2007), which have shown increased accuracy over individual classifiers for detecting and mapping dead wood.

\subsubsection{Field Validation}

Validation of the map was carried out using sites that were completely independent of the creation of the map itself. That is, any wood objects that were used as training data (e.g. for the unmixing endmembers and objected-based classification) were not used for validation. To test the map accuracy in terms of its ability to identify the presence of dead wood, fifty dead wood objects in the map were selected and validated in the field. They were spatially distributed within three regions across the forest for 
accessibility and to be representative of the whole study area. They were also selected to represent the range of dead wood object sizes in the final map. In order to test the map's accuracy in terms of its representation of the absence of dead wood, and examine any errors of commission, a set of sites (hereafter called control points) were selected in the forest, which according to the map showed no detected dead wood. Since there was some positional error caused by image georeferencing and the GPS, a $10 \mathrm{~m}$ radius buffer was used around each control point (i.e. a control point was selected if no dead wood was present on the map within $10 \mathrm{~m}$ ). Only thirty control sites were used since dead wood objects were quite numerous and widely distributed and locations that specifically had no dead wood present within $10 \mathrm{~m}$ were of interest, which eliminated many potential sites.

The locations of all of the test wood objects $(n=50)$ and control points $(n=30)$ were randomized and loaded into a real time differential GPS that provided approximately $1 \mathrm{~m}$ horizontal accuracy. The identity of each point in terms of whether it was a delineated wood object or a control point was unknown to the researchers while they were in the field, with only a random identification number used to link the points to their known attributes that could be looked up following a visit to the field. This created a set of pseudo-independent and unbiased test points that were visited in the field without any a priori knowledge of whether the specific location in the field was mapped as a dead tree or with no significant wood object detected (i.e. control site).

Using the GPS with a $4 \mathrm{~m}$ external antenna, which ensured that enough satellites were used to maintain a stable signal with a low signal-to-noise ratio, the general location of each point was visited by researchers and small movements were made within the 
general vicinity of the locations in order to match the researchers' location with the location of the validation points on the GPS screen. Once the locations remained stable within a couple of meters for several minutes, the two researchers scanned the forest (in a radius of about $10 \mathrm{~m}$ to account for any image registration and GPS error) and recorded the presence or absence of any dead trees as well as other pertinent information about the surroundings that could potentially aid the interpretation of the results. While no specific size threshold was used to decide whether a dead tree was present, any small dead trees (i.e. below the overstorey) that were found were examined and recorded to see if they were within a canopy gap and could therefore potentially be detected in the imagery.

\subsubsection{Map of Dead Wood Spatial Distribution}

A final map of the spatial distribution of dead wood across the forest was produced, similar to the methods presented by Meentemeyer et al. (2008), by calculating the total number of wood pixels found within a $400 \mathrm{~m}^{2}$ area, the same area as the field plots used throughout the research.

\subsubsection{Indirect Regression Modelling of Dead Wood}

For regression-based modeling of dead wood a plot-based approach was taken. As previously discussed in Section 4.3 .1 fifty $20 \mathrm{~m}$ x $20 \mathrm{~m}$ field plots were established across the study area. As outlined in Section 4.3.2.4, among other variables measured and calculated in these plots, the number of snags in each plot was recorded, along with the number of pieces and approximated volume of CWD (diameter $>7.5 \mathrm{~cm}$ ). 
Separate forward stepwise multiple regressions were carried out in order to attempt to explain the variance of these field variables using a variety of image predictor variables. The plot-based variables that had been extracted from the airborne imagery (Section 4.3.3), including spectral, spatial, and object-based information, as well as topographic information derived from a digital elevation model were tested for their explanatory capabilities. Information extracted specifically from the red and NIR bands was expected to be representative of the presence and abundance of dead wood in the plots based on spectral reflectance characteristics of exposed wood compared to the surrounding foliage. Additionally, following the assumption that heterogeneity in the canopy caused by canopy gaps and the presence of dead wood would be visible in the imagery, image spatial information was also used as a source of possible predictor variables. Object-based information extracted from the crowns and shadows within the plots, which potentially captured structural conditions not represented by the spectral and spatial variables, was also tested. 


\subsection{Results}

\subsection{Development of an Image-based Structural Complexity Index}

\subsubsection{Field Variables Representing Structural Attributes}

The fifty field plots selected across the study area provided a wide range of structural conditions. Out of the fifty field plots, ten were identified (qualitatively) as having relatively low structural complexity and eleven were identified as having relatively high structural complexity. The remaining twenty-nine plots that fell within a moderate complexity class, showing what was considered typical structural conditions in this area of the hardwood forest. These divisions were simply qualitative and never used for any quantitative analysis (for example a Linear Discriminant Analysis) since the continuous variables that had been measured in the plots were used for the Redundancy Analysis. Conditions in the plots with low structural complexity included areas that had homogeneous overstorey and no ground vegetation or CWD, as well as areas with homogeneous overstorey and continuous low ground vegetation covering the forest floor with little CWD (Figure $10 \mathrm{~A}$ and B). Plots qualitatively classified as having relatively high structural complexity typically had large canopy gaps, variable overstorey tree sizes, and piles of CWD mixed with variable ground and understorey vegetation (Figure $10 \mathrm{C}$ and D). Hemispherical photographs taken at $1 \mathrm{~m}$ and $6 \mathrm{~m}$ heights provided evidence of the thick understorey and large canopy gaps typically present in high structural complexity areas with a large difference between the photos taken at the two different heights, and as a result, significant differences in canopy openness and LAI measurements (Figure 11). 
Within the field plots, a total of twenty-four variables, each representing different structural attributes of the forest were tabulated (Table 3 (actual plot data included in Appendix 3)). As an overview, $82 \%$ of the 1055 overstorey trees that were measured were maples; either sugar (Acer saccharum) or red (Acer rubrum). Some notable relations and interesting statistics were observed. As expected, as average tree dbh increased the stem density decreased $(r=-0.70, p<0.001)$ and the average tree height increased $(r=0.76, p<0.001)$. As well, the plots exhibited variable tree spacing showing a variation in the horizontal distributions of the overstorey trees $(1-3.2 \mathrm{~m})$. Within the plots, vegetation $10-50 \mathrm{~cm}$ tall (generally ferns and small shrubs), as well as understorey vegetation $>2 \mathrm{~m}$ tall (generally maple saplings), dominated the vertical height profiles with average coverages of $27.8 \%$ and $20.5 \%$ respectively, while average coverages of $0-10 \mathrm{~cm}, 50 \mathrm{~cm}-1 \mathrm{~m}$, and $1-2 \mathrm{~m}$ tall ground vegetation were $5.8 \%, 6.7$ $\%$, and $8.1 \%$ respectively. Finally, canopy openness (measured at $6 \mathrm{~m}$ ) was found to be positively correlated with the coverage of understorey vegetation (saplings) $(r=0.42, p<$ 0.001), reflecting the relation between incoming light and canopy gaps with respect to vegetation growth beneath the canopy. The estimated volume of CWD was found to be so highly correlated with the number of pieces counted $(r=0.92, p<0.001)$ that it was not included in the modelling, however it was retained and used for specifically modelling forest dead wood.

This dataset was found to have high dimensionality, with a Principal Components Analysis (PCA) producing nine components with eigenvalues greater than 1.0 indicating that the components summarized more information than any single original variable (Kaiser, 1960; Jackson, 1993). 

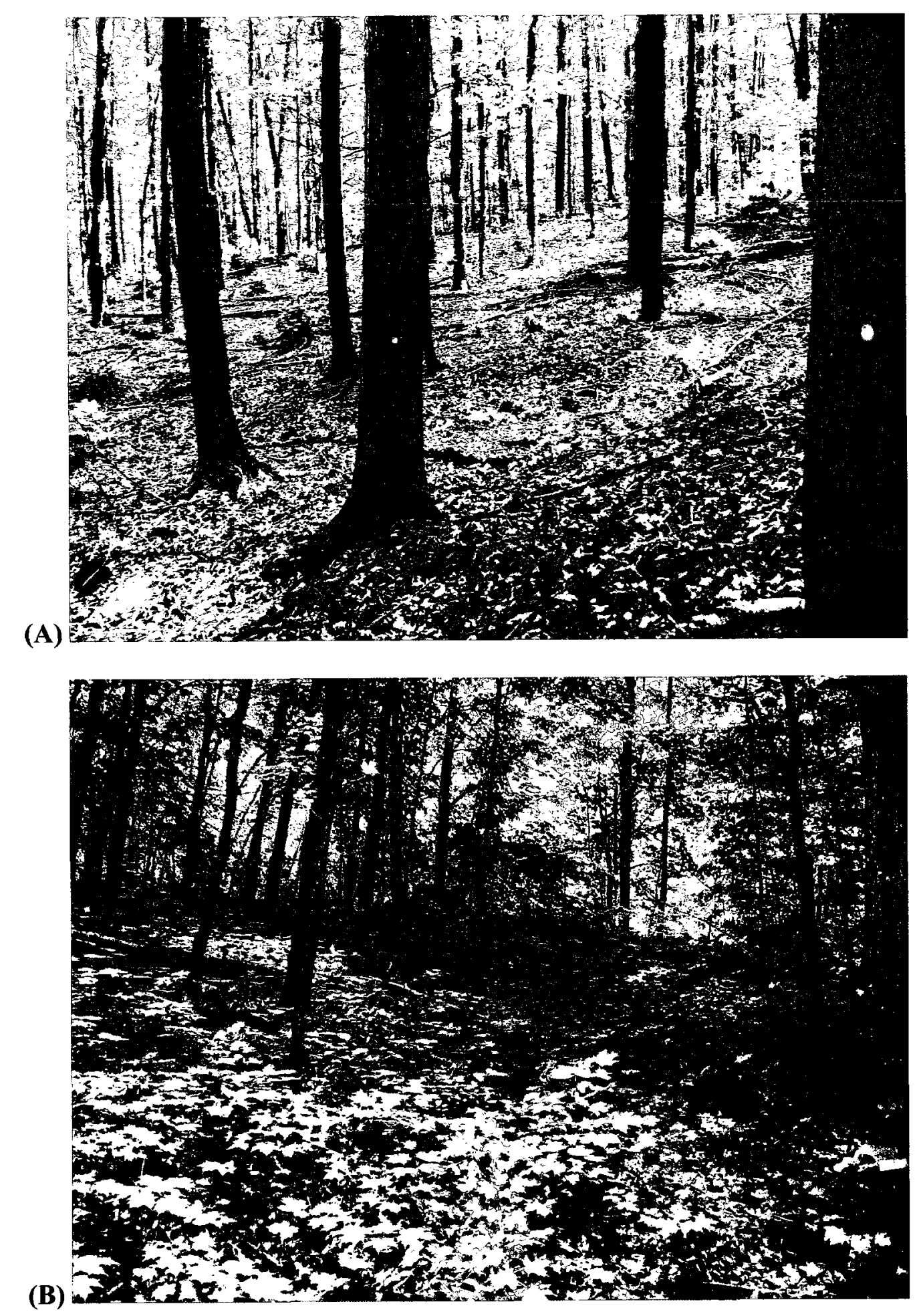

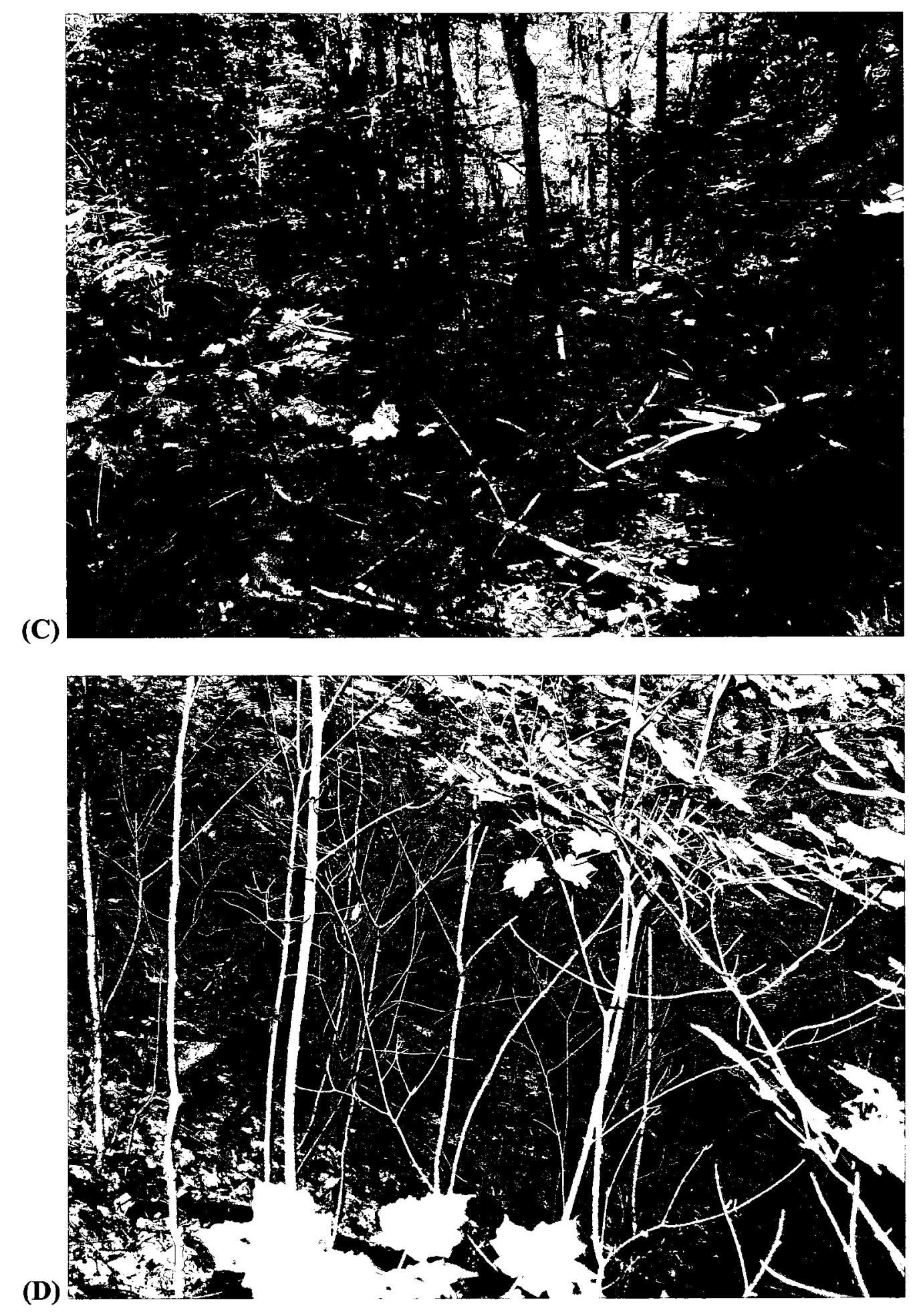

Figure 10. Structural conditions typical of area of relatively low structural complexity (A (Plot 2) and B (Plot 35)) and relatively high structural complexity (C (Plot 43) and D (Plot 12)) within the study area. 

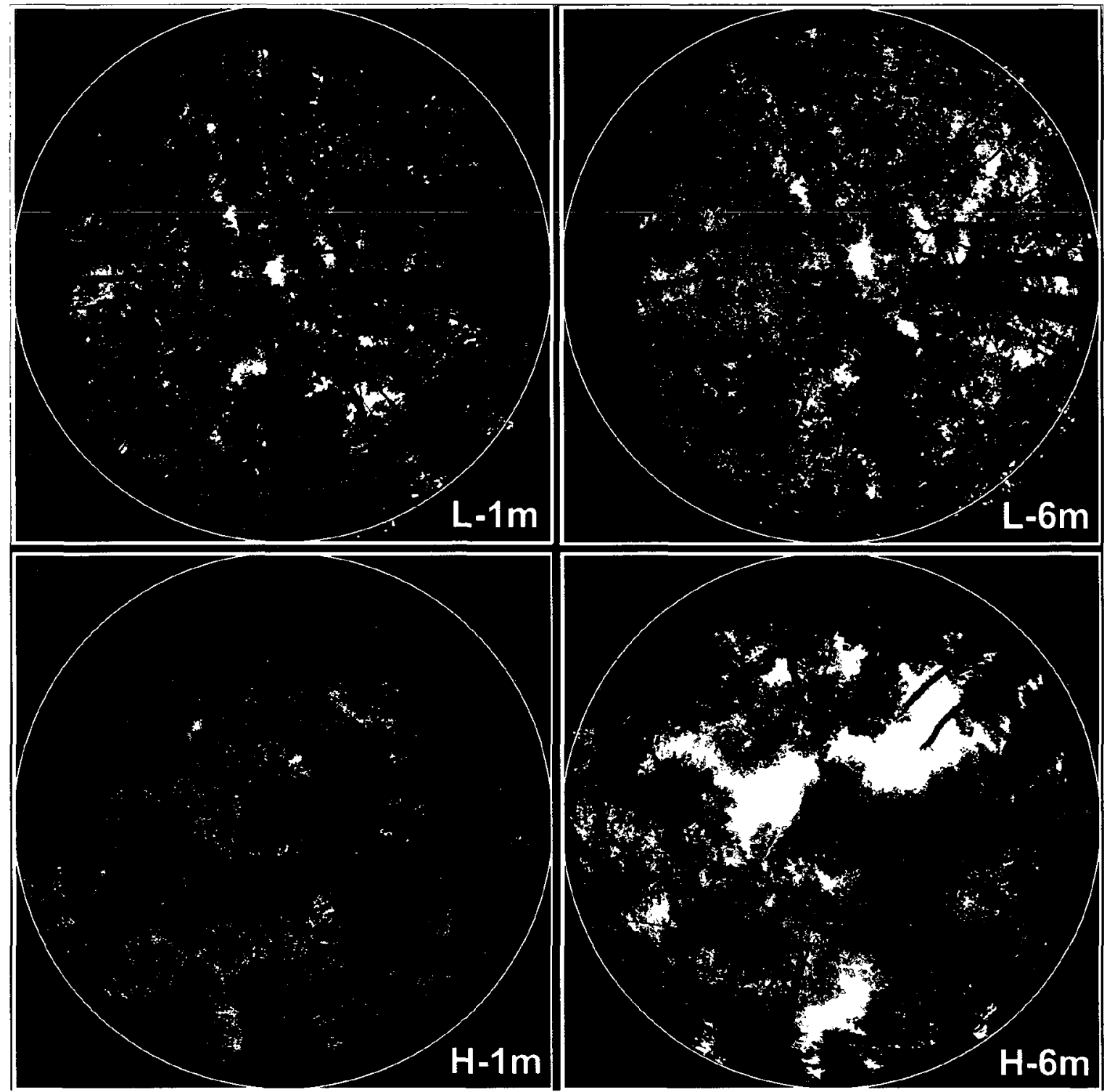

Figure 11. Example hemispherical photographs taken in a plot with relatively low complexity (Plot 2) (L) as well as one with relatively high complexity (Plot 20) (H). Large differences between the $1 \mathrm{~m}$ and $6 \mathrm{~m}$ photograph can be seen in the high complexity plot as a result of the thick understorey vegetation $(\mathrm{H}-1 \mathrm{~m})$ and the large canopy gaps $(\mathrm{H}-6 \mathrm{~m})$. The opposite was generally true in the low complexity plots, where little understorey vegetation was present to block the camera lens (L-1m) and few significant canopy gaps existed (L-6m). 
Table 3. Final set of field variables collected and calculated within each field plot. Summary statistics shown for each. $(\mathrm{n}=50)$

\begin{tabular}{|c|c|c|c|c|}
\hline Variable & Minimum & Maximum & Average & $\begin{array}{l}\text { Standard } \\
\text { Deviation }\end{array}$ \\
\hline Number of Overstorey Trees & 5.00 & 42.00 & 21.10 & 9.29 \\
\hline Average Overstorey Tree $\mathrm{dbh}(\mathrm{cm})$ & 16.93 & 33.29 & 24.32 & 4.18 \\
\hline $\begin{array}{l}\text { Standard Deviation of Overstorey } \\
\text { Tree } \mathrm{dbh}(\mathrm{cm})\end{array}$ & 3.85 & 17.17 & 9.21 & 2.71 \\
\hline Live Basal Area ( $\left.\mathrm{m}^{2} / \mathrm{ha}\right)$ & 8.17 & 47.26 & 25.99 & 7.30 \\
\hline Number of Large Trees & 0.00 & 6.00 & 2.14 & 1.63 \\
\hline $\begin{array}{l}\text { Standard Deviation of Overstorey } \\
\text { Tree Height }(\mathrm{m})\end{array}$ & 2.24 & 6.97 & 4.17 & 1.32 \\
\hline Average Nearest Neighbour Distance (m) & 0.99 & 3.18 & 1.95 & 0.55 \\
\hline Average Overstorey Crown Diameter $(\mathrm{m})$ & 2.94 & 5.40 & 4.03 & 0.48 \\
\hline $\begin{array}{l}\text { Standard Deviation of Overstorey Crown } \\
\text { Diameter }(\mathrm{m})\end{array}$ & 0.07 & 2.82 & 1.15 & 0.41 \\
\hline Number of Snags & 1.00 & 19.00 & 4.88 & 3.41 \\
\hline Average Snag dbh $(\mathrm{cm})$ & 11.97 & 49.34 & 22.88 & 7.68 \\
\hline Dead Basal Area $\left(\mathrm{m}^{2} / \mathrm{ha}\right)$ & 0.30 & 19.15 & 5.49 & 4.10 \\
\hline Number of Pieces of CWD & 1.00 & 30.00 & 12.14 & 6.86 \\
\hline $0-10 \mathrm{~cm}$ Ground Vegetation (\% of plot) & 0.00 & 25.00 & 5.75 & 6.27 \\
\hline $10-50 \mathrm{~cm}$ Ground Vegetation (\% of plot) & 1.79 & 90.18 & 27.77 & 19.89 \\
\hline $50 \mathrm{~cm}-1 \mathrm{~m}$ Ground Vegetation (\% of plot) & 0.00 & 33.04 & 6.68 & 6.52 \\
\hline $1 m-2 m$ Ground Vegetation (\% of plot) & 0.00 & 29.46 & 8.05 & 7.84 \\
\hline$>2 \mathrm{~m}$ Ground Vegetation ( $\%$ of plot) & 0.00 & 97.32 & 20.45 & 23.82 \\
\hline $1 \mathrm{~m}$ Openness $\left(0-60^{\circ}\right)(\%)$ & 1.00 & 14.50 & 6.01 & 3.82 \\
\hline $1 \mathrm{~m} \mathrm{LAI}\left(55-60^{\circ}\right)$ & 3.06 & 6.89 & 5.41 & 0.95 \\
\hline $6 \mathrm{~m}$ Openness $\left(0-60^{\circ}\right)(\%)$ & 3.30 & 45.50 & 15.61 & 8.77 \\
\hline $6 \mathrm{~m} \mathrm{LAI}\left(55-60^{\circ}\right)$ & 2.42 & 6.08 & 4.29 & 0.93 \\
\hline Change in Openness $\left(0-60^{\circ}\right)(\%)$ & -2.30 & 37.10 & 9.59 & 9.34 \\
\hline Change in $\mathrm{LAI}\left(55-60^{\circ}\right)$ & -4.00 & 1.20 & -1.12 & 1.28 \\
\hline
\end{tabular}




\subsubsection{Image and Topographic Variables (Explanatory / Predictor Variables)}

In total there were eighty-six predictor variables extracted from the airborne imagery as well as the digital elevation model, with each potentially providing new information, which was of particular interest to this project. The image dataset was found to also have very high dimensionality, with a PCA showing that while many variables were correlated with one another, eleven components were found to have eigenvalues greater than 1.0 .

\subsubsection{Modelling Structural Complexity with Redundancy Analysis}

The five-hundred models produced using the RDA bootstrapping analysis included between one and fourteen predictor variables $(\overline{\mathrm{x}}=3.12)$ with model adjusted $\mathrm{R}^{2}$ values ranging from 0.17 to $0.48(\overline{\mathrm{x}}=0.29)$ (Table 4$)$. The statistics presented in this table were calculated for each of the 500 models and used to rank the models in order to select a final model for further testing. Note that the variable r-validate is simply a correlation coefficient and unlike the adjusted $\mathrm{R}^{2}$ does not take into account the number of predictors used. As a direct consequence of this the squared value of r-validate are greater than the adjusted $\mathrm{R}^{2}$ values presented. The models were made up of various combinations of predictor variables that contributed to explaining the variance in the set of field data (Table 5). Most notably, these results showed that $70 \%$ of the models included measures of within-crown texture. Topographic information and semivariogram range and sill were included in $32 \%$ and $28 \%$ of the models, respectively. Beyond that, within-crown spectral information, within-shadow spectral and spatial information and object-based 
information contributed moderately to the models, with full plot spectral and spatial information contributing very little to the models.

Table 4. Summary statistics calculated from the 500 models (all $p<0.001$ ) that were produced from the first phase of bootstrapping in RDA, which was carried out for variable selection.

\begin{tabular}{|c|c|c|c|c|c|}
\hline & $\begin{array}{l}\text { Adjusted } \\
\mathbf{R}^{2}\end{array}$ & $\begin{array}{c}\text { r- } \\
\text { Validate* }\end{array}$ & $\begin{array}{c}\text { \# of } \\
\text { Predictors }\end{array}$ & \% RMSE & AIC \\
\hline Average & 0.29 & 0.69 & 3.12 & 25.6 & 349.4 \\
\hline Minimum & 0.17 & 0.60 & 1.00 & 17.6 & 344.7 \\
\hline Maximum & 0.48 & 0.83 & 14.00 & 38.3 & 361.5 \\
\hline
\end{tabular}

Table 5. Results of bootstrapping analysis in terms of sources / types of predictor variables included in the different models (total number of models produced was 500).

\begin{tabular}{lcc}
\hline & $\begin{array}{c}\text { \% of All } \\
\text { Models }\end{array}$ & $\begin{array}{c}\text { \# of } \\
\text { Models }\end{array}$ \\
\hline Within Crown GLCM Texture & 70 & 348 \\
Topographic & 32 & 159 \\
Semivariogram Range and Sill & 28 & 138 \\
Within Crown Spectral & 22 & 112 \\
Within Shadow $1^{\text {st }}$ Order Spatial & 21 & 106 \\
Object-based & 19 & 94 \\
Within Shadow GLCM Texture & 18 & 90 \\
Full Plot GLCM Texture & 11 & 54 \\
Subpixel Fractions & 10 & 50 \\
Within Shadow Spectral & 8 & 40 \\
Within Crown 1 $1^{\text {st }}$ Order Spatial & 2 & 11 \\
Full Plot 1 $^{\text {st }}$ Order Spatial & 1 & 6 \\
Full Plot Spectral & 1 & 5 \\
\hline
\end{tabular}


Within the groups of variables there were some notable results and it was found that often two related variables were exchanged for one another in different models. Average NIR reflectance within-crowns was entered in 81 out of 112 models that included within-crown spectral information. Of the 348 models that included withincrown GLCM texture variables, 316 included the standard deviation of Homogeneity, while 110 included the average Correlation, of which 96 of the models included both. Of the within-shadow GLCM texture variables, the standard deviation of Correlation was included in 70 out of the 90 models. The ranges of the NIR and Green bands were included in 65 and 51 models respectively, while the sills from each of the three bands were only included in a combined 7 out of the 138 models that included semivariogram measures. With respect to topographic information, elevation was included in 95 out of the 159 models, while the variable representing illumination was included in 58 models.

The top ranked models, based on the results from the validation samples in the bootstrapping analysis, had nine predictor variables, which was deemed to be too many given there were only fifty field plots in the model. For RDA, which is similar to a sequential combination of Canonical Correlation Analysis (CCA) and multiple regression, the number of observations to the number of variables was only $40: 9$. This was considered to be an unacceptable ratio based on the commonly used rule of thumb suggesting at least a $10: 1$ ratio (e.g. Tabachnick and Fidell, 1996; Babyak, 2004).

The $5^{\text {th }}$ through $8^{\text {th }}$ ranked models were the next best and included six predictor variables (Adjusted $\mathrm{R}^{2}=0.40 ; \mathrm{RMSE}=20 \%$ ). These models all included the same predictor variables, and were therefore simply different instances of a single model. The 
model's $1^{\text {st }} \mathrm{RDA}$ axis $(\mathrm{RDA} 1)$ had an adjusted $\mathrm{R}^{2}$ of $0.38(\mathrm{p}<0.001)$. This first axis was defined as a Structural Complexity Index (SCI) based on its representation of a structural complexity gradient defined by the field measurements. An examination of the bivariate correlations between RDA1 and the individual field variables, as well as an examination of the biplot scores of the image variables included in this model (see Section 5.1.4.2), showed that structural complexity increased as: (1) the variation of GLCM Homogeneity within-crowns increased, (2) the range of the green band semivariogram increased, (3) the number of delineated canopy objects decreased, (4) the average GLCM Correlation within-crowns increased, (5) the variation in green band shadow reflectance decreased, and (6) illumination increased.

These results showed that increasing heterogeneity in the canopy, represented in different ways by three image spatial variables, fewer canopy objects suggesting larger crowns and larger gaps in the canopy, as well deeper and more uniform between-crown shadows, were all associated with increasing structural complexity as measured in the field plots. While these results were interesting from a research point of view, for the practical goal of structural complexity mapping, a model was desired that included simpler variables than semivariogram range, which was complicated and computationally intensive to calculate for every pixel across the image in order to produce a continuous map.

\subsubsection{Final RDA Model}

The final RDA model (Table 6) that was selected did not include semivariogram measures as a result of the tradeoff between computational simplicity and explanation. 
The semivariogram measures were also found to be correlated with many other variables, with many bivariate correlations found to be greater than 0.6 . This final model had slightly lower explanation (only $3 \%$ less) of the variation in the field variables (Adjusted $\mathrm{R}^{2}$ of RDA1 $\left.=0.35, \mathrm{p}<0.001\right)$ yet still provided a meaningful and highly significant model. It also satisfied a $10: 1$ ratio by incorporating five predictor variables when the final model, including all fifty field plots, was run. Only the first RDA axis was of interest. The second RDA axis (y axis) provided a further $6 \%$ explanation $(\mathrm{p}=0.080)$, found by using the first RDA axis as a co-variable. This first axis (RDA1) was defined as a Structural Complexity Index (SCI) based on interpretation of the gradient.

Table 6. Summary results from selected RDA (produced by CANOCO).

\begin{tabular}{lccccc}
\hline Axes variance: & $\mathbf{1}$ & $\mathbf{2}$ & $\mathbf{3}$ & $\mathbf{4}$ & Total \\
Eigenvalues: & 0.35 & 0.06 & 0.02 & 0.01 & 1.00 \\
Field-image correlations: & 0.81 & 0.67 & 0.50 & 0.33 & \\
Cumulative percentage variance & & & & & \\
$\quad$ Of field data: & 34.5 & 40.6 & 42.6 & 43.7 & \\
$\quad$ Of field-image relation: & 77.7 & 91.6 & 95.9 & 98.5 &
\end{tabular}

Sum of all eigenvalues:

Sum of all canonical eigenvalues:

Test of significance of first canonical axis:

Eigenvalue $=0.35$, F-ratio $=23.17$, $P$-value $<0.001$ 
A major benefit of using RDA was that it had the ability to produce a triplot (Figure 12) which was used to improve understanding of the model, aid in the interpretation of relations between variables (both field and predictor), and aid in the visual analysis of how the different field plots were positioned on the structural complexity axis. The triplot for the selected model, which was created using CanoDraw (CANOCO, ter Braak and Šmilauer, 2002) provided various types of information. The five predictor variables (IM1 to IM5) are displayed on the triplot as red arrows, with their length and direction, relative to the SCI (x axis) indicative of their contribution to the SCI. Each field variable $(n=24)$ is displayed on the triplot as a blue arrow with field variables that are positively correlated with one another drawn close to each other (e.g. number of overstorey trees and live basal area, $\mathrm{r}=0.60, \mathrm{p}<0.001$ ). Arrows that point in opposite directions to each other are negatively correlated (e.g. number of overstorey trees and mean crown diameter, $\mathrm{r}=-0.46, \mathrm{p}<0.001$ ) and arrows that approach right angles to one another are not correlated at all (e.g. number of overstorey trees and standard deviation of crown diameter, $r=0.01, p=0.963$ ). Similarly, relations between field variables (blue arrows) and predictor variables (red arrows) can be inferred based on the directions of the arrows. For example, IM3, representing green photosynthetic vegetation within canopy gaps, was positively correlated with the field variables $1 \mathrm{~m} \mathrm{LAI}$ $(\mathrm{r}=0.34, \mathrm{p}=0.018)$ as well as $>2 \mathrm{~m}$ understorey vegetation $(\mathrm{r}=0.33, \mathrm{p}=0.018)$, represented by arrows close to IM3.

Finally, displaying the fifty field plots on the triplot (the $3^{\text {rd }}$ element of the triplot), using the scores for each axis, allowed the field plots to be visually assessed in terms of their individual structural attributes. For example, Plots \# 37, \# 40, and \# 44 had among 
the highest number of overstorey trees and low SCI values while Plot \# 50 had the highest SCI value controlled in large part by the fact that this plot had relatively few overstorey trees, which were of variable sizes (dbh and height), large canopy gaps and abundant understorey vegetation (covering $97.3 \%$ of the plot). The plots are shown in colours reflective of the preliminary, and qualitative, classification that was assigned to each plot when they were originally established (see Section 4.3.1).

For the most part plots labeled as relatively low complexity (shown as green dots in Figure 12) were located on the far left of the triplot at the lower end of the SCI gradient. Plots labeled as relatively high complexity (red dots) were for the most part located on the far right of the triplot, exhibiting high values along the SCI gradient. In order to simplify this comparison, the fifty field plots were plotted on a scatterplot classified in these original three qualitiative classes (low, medium, and high) compared to the predicted SCI score for each plot from the RDA (Figure 13). This figure provides confirmation that indeed most plots associated with low and high complexity were distinguishable from the rest. However, overlap was seen between low and medium complexity plots and similarly medium and high complexity plots. Interestingly, nine out of the fifty plots showed confusion between the three classes (shown circled in the figure), which although not directly related happened to correspond very well with the expected error of the RDA model (19.9 \% RMSE). This seemed to suggest that the modelling procedures could potentially identify areas of the forest with very high or very low structural complexity quite accurately. 


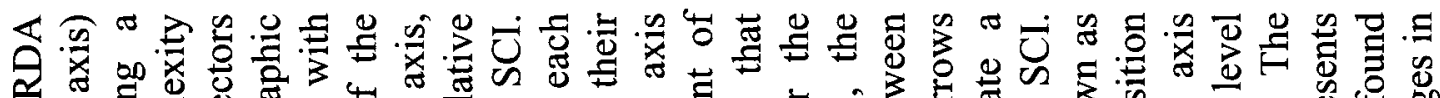

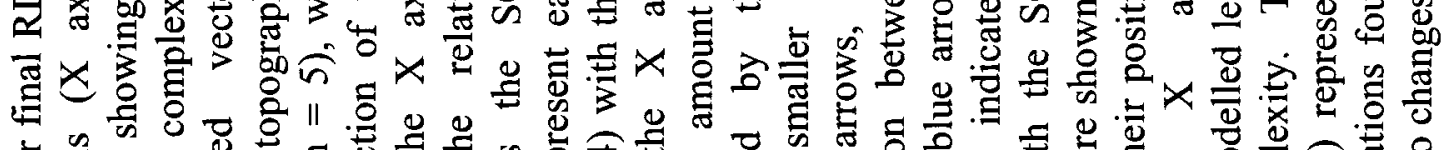

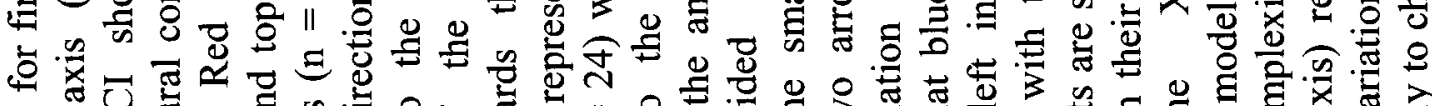

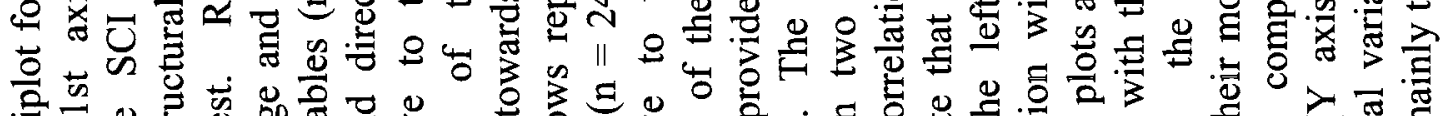

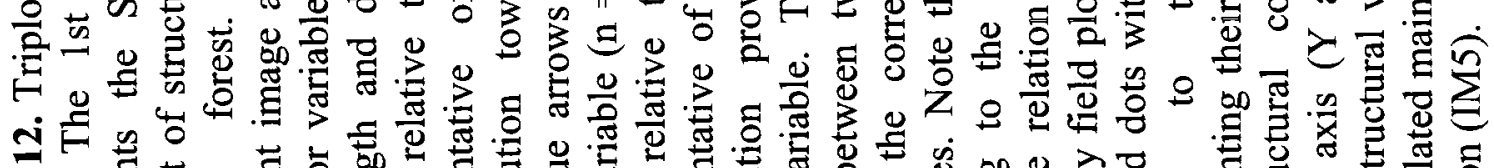

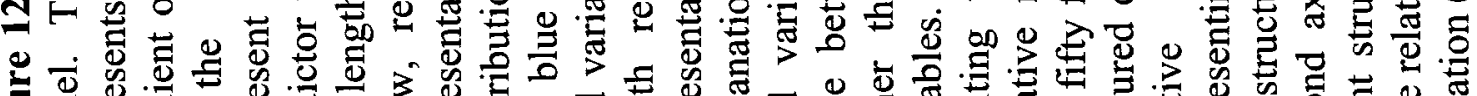

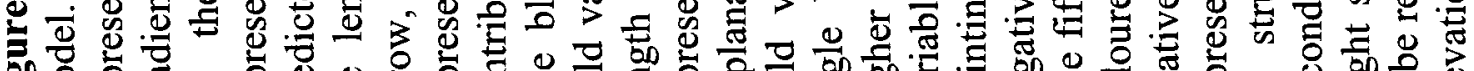

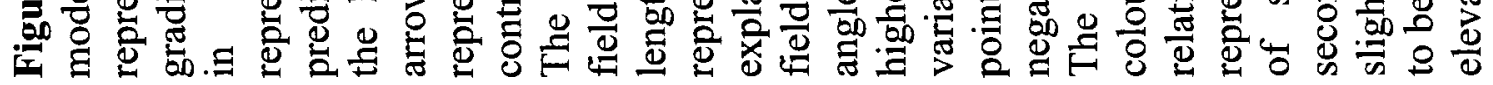

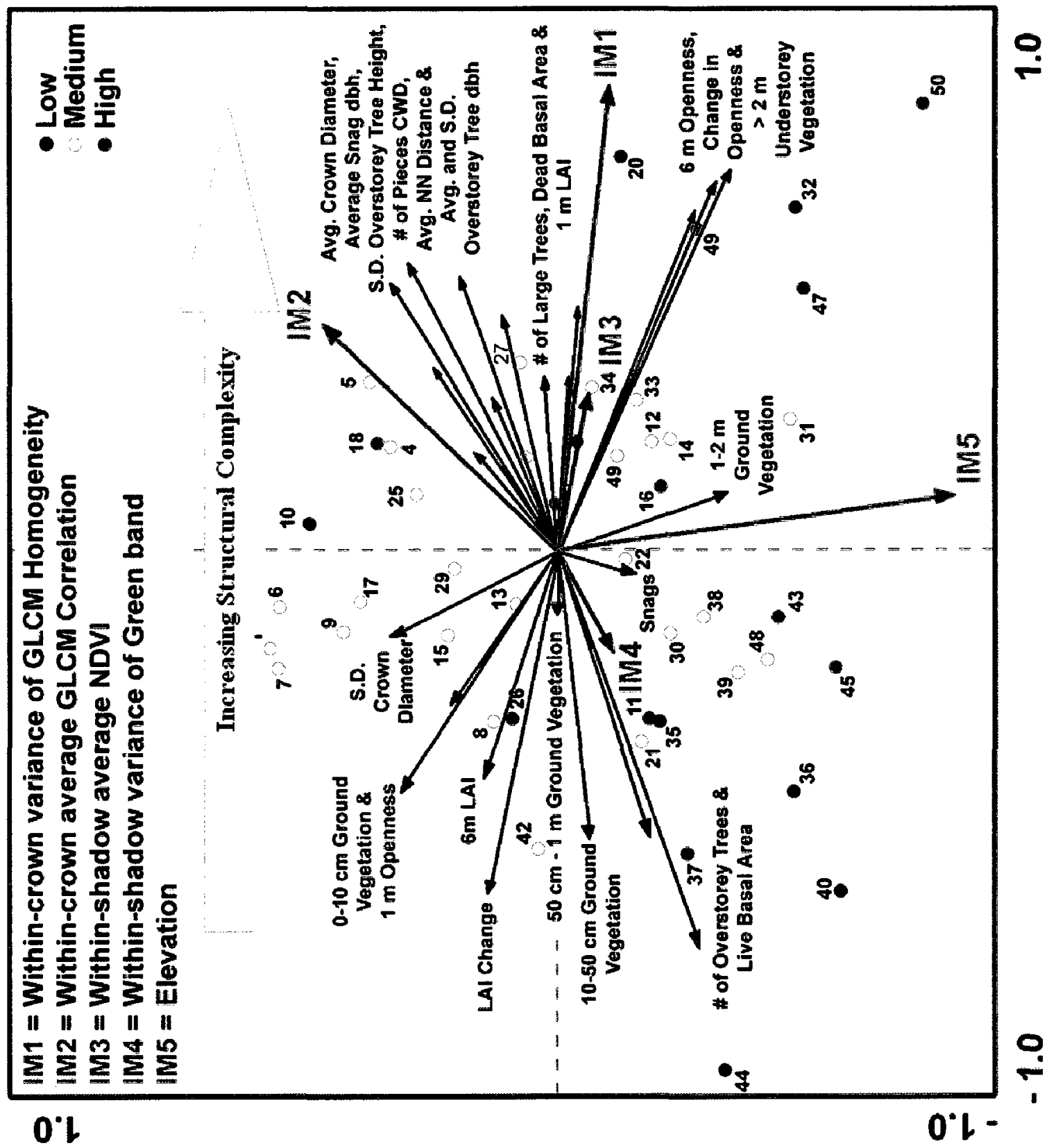




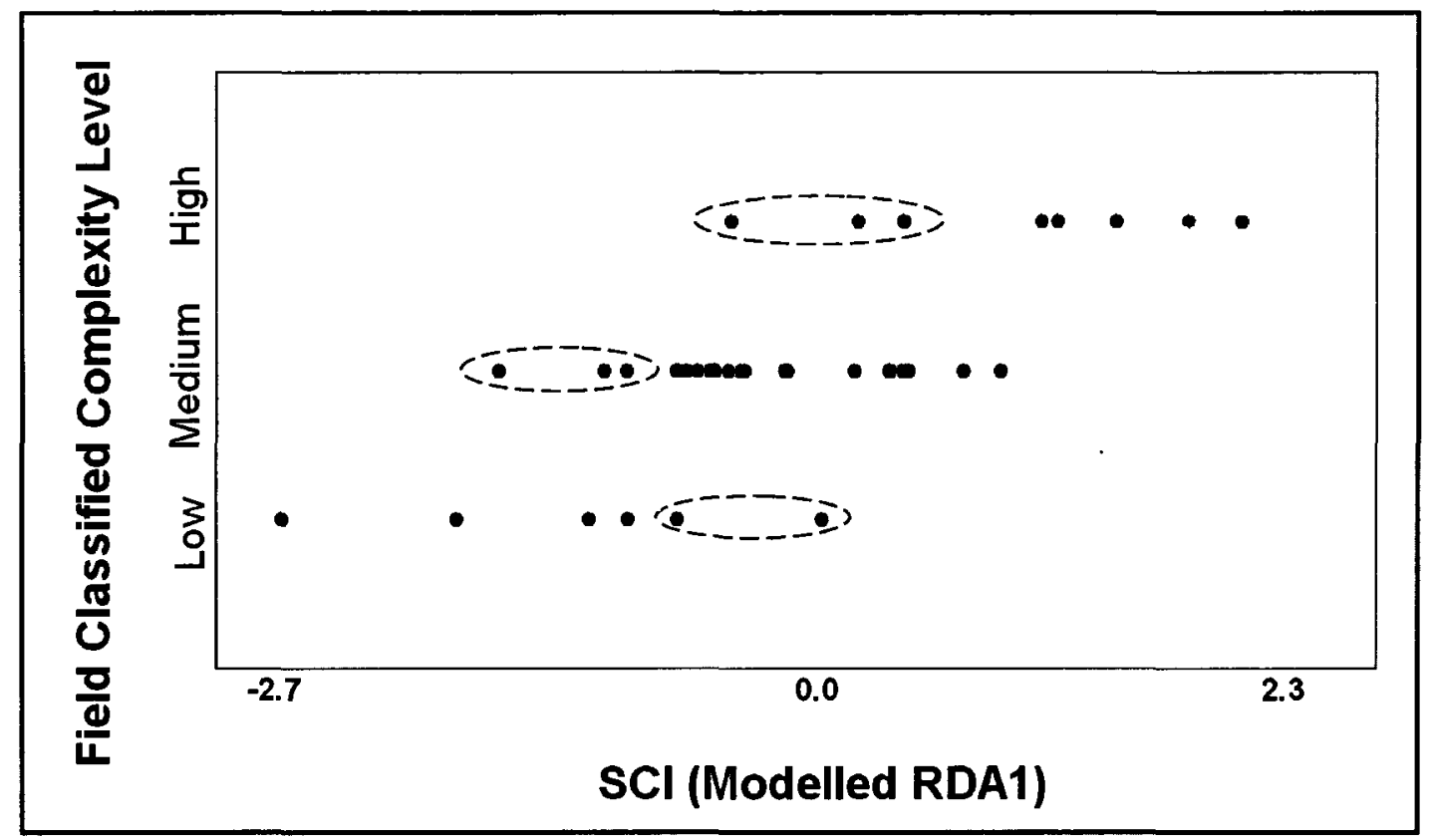

Figure 13. Relation between the modeled SCI, shown using a standardized scale from lowest SCI (-2.7) to highest SCI (2.3), and the original qualitative complexity level associated with each field plot through visual assessment. Field plots that showed confusion between classes are circled for each complexity class (i.e. low, medium, high).

\subsubsection{Interpreting the SCI from a Field Perspective}

An examination of the bivariate correlations between the SCI and the individual field variables (Table 7) provided a detailed explanation of the representation of the SCI in terms of a gradient of structural complexity. Scatterplots of these relations are shown in Appendix 4. Generally, the gradient can be best interpreted by looking at the sign of the significant correlations. For example, increased canopy openness and understorey vegetation $(>2 \mathrm{~m})$, and conversely decreased low ground vegetation $(0-50 \mathrm{~cm})$, corresponded to a greater value of SCI. From a biodiversity perspective, this finding supports the results of others including Carey (2003) who found that, in the short term, the introduction of heterogeneity into homogeneous canopies can have positive effects on 
the diversity of biotic communities. The increased understorey vegetation, a result of elevated understorey light levels caused by increased canopy openness (Canham, 1988; Decocq et al., 2004) provided vertical heterogeneity in certain study plots. This was predominantly from maple saplings that had benefited from canopy damage that was caused by the 1998 ice storm. King and Bemrose (2005) showed that plots in Gatineau Park that experienced greater damage from the ice storm generally showed little increase in ground vegetation between 1998 and 2000. These results were supported in this research, which showed more complex plots to have less ground vegetation, most likely caused by the presence of the saplings which out-competed the ferns and herbs and quickly blocked the light from reaching the ground vegetation.

The SCI was also higher where there were larger snags (average snag dbh), more dead basal area and a greater number of CWD pieces. There is no doubt regarding the benefits of both standing dead trees and woody debris on the forest floor in terms of increasing structural complexity of the forest and providing habitat and increased biodiversity. Snags have been found essential to birds for nesting and feeding (e.g. Stribling, 1990; Spiering and Knight, 2005) and as shelter for a variety of mammals (e.g. Payer and Harrison, 2003; Smith, 2007). In fact, Holloway et al. (2007), investigating snags in hardwood forests in central Ontario, found nesting and feeding cavities to be twice as likely to occur in snags compared to live trees, with an increased probability of cavities in snags with greater dbh. Meanwhile, CWD, which comes directly from dead or dying trees, provides breeding habitat and shelter for many small vertebrates (e.g. Maguire, 2002; McKenny et al., 2006) and invertebrates (e.g. Hammond et al., 2001; McGeoch et al., 2007). 
Table 7. Correlation coefficients between field variables and SCI ( $1^{\text {st }} \mathrm{RDA}$ axis) shown in order from strongest to weakest.

\begin{tabular}{|c|c|c|}
\hline Field Variables & Pearson r & $\mathbf{p}$ \\
\hline Number of Overstorey Trees & -0.72 & $<0.001$ \\
\hline Change in Openness $\left(0-60^{\circ}\right)(\%)$ & 0.70 & $<0.001$ \\
\hline$>2 \mathrm{~m}$ Ground Vegetation ( $\%$ of plot) & 0.67 & $<0.001$ \\
\hline Change in LAI $\left(55-60^{\circ}\right)$ & -0.63 & $<0.001$ \\
\hline $6 \mathrm{~m}$ Openness $\left(0-60^{\circ}\right)(\%)$ & 0.62 & $<0.001$ \\
\hline Average Snag dbh (cm) & 0.53 & $<0.001$ \\
\hline $10-50 \mathrm{~cm}$ Ground Vegetation (\% of plot) & -0.52 & $<0.001$ \\
\hline Live Basal Area $\left(\mathrm{m}^{2} / \mathrm{ha}\right)$ & -0.51 & $<0.001$ \\
\hline Average NN Distance (m) & 0.50 & $<0.001$ \\
\hline Standard Deviation of Overstorey Tree Height (m) & 0.49 & $<0.001$ \\
\hline $1 \mathrm{~m} \mathrm{LAI}\left(55-60^{\circ}\right)$ & 0.45 & $<0.001$ \\
\hline $0-10 \mathrm{~cm}$ Ground Vegetation ( $\%$ of plot) & -0.43 & 0.002 \\
\hline Average Overstorey Tree $\mathrm{dbh}(\mathrm{cm})$ & 0.43 & 0.002 \\
\hline 6 m LAI $\left(55-60^{\circ}\right)$ & -0.41 & 0.003 \\
\hline Number of Pieces of CWD & 0.34 & 0.017 \\
\hline Dead Basal Area (m²/ha) & 0.32 & 0.022 \\
\hline Number of Large Trees & 0.32 & 0.025 \\
\hline $1 \mathrm{~m}$ Openness $\left(0-60^{\circ}\right)(\%)$ & -0.28 & 0.051 \\
\hline Standard Deviation of Overstorey Tree $\mathrm{dbh}(\mathrm{cm})$ & 0.28 & 0.052 \\
\hline Average Overstorey Crown Diameter (m) & 0.18 & 0.211 \\
\hline $50 \mathrm{~cm}-1 \mathrm{~m}$ Ground Vegetation (\% of plot) & -0.12 & 0.423 \\
\hline $1 m-2 m$ Ground Vegetation (\% of plot) & 0.11 & 0.461 \\
\hline Standard Deviation of Overstorey Crown Diameter (m) & -0.05 & 0.735 \\
\hline Number of Snags & -0.04 & 0.782 \\
\hline
\end{tabular}


Additionally, an increase in the size of overstorey trees (average tree dbh and number of large trees) and a decrease of the number of those trees (number of overstorey trees and live basal area) corresponded to a greater value of SCI. Larger trees have been found to have a much higher probability of containing nests and providing feeding cavities. For example, Holloway et al. (2007) showed a significant increase in these features in live trees $>35 \mathrm{~cm}(\mathrm{dbh})$, which matched the definition of large trees used in this study, as well as a sharp increase in cavities, including ones used for shelter, in trees with $>45 \mathrm{~cm} \mathrm{dbh}$, demonstrating the importance of large trees for habitat.

Interestingly, five of the twenty-four field variables were uncorrelated with the SCI. The measures of crown diameter were known to have significant error simply due to the measurement methods (tests showed $\sim 14 \%$ standard deviation compared to the range from repeated measurements) and therefore may have been too noisy or had poor fit with the other variables measured in the ecosystem. Another variable, $1-2 \mathrm{~m}$ ground vegetation, was positively correlated with the second RDA axis, however that axis was not found to be significant.

Perhaps most interesting to forest structural complexity modelling with regards to biodiversity was the fact that the actual number of snags counted in the field plots was completely uncorrelated with the SCI $(r=-0.04, p=0.782)$. This suggests that the number of snags in the forest was not strongly related to the other structural attributes measured in the ecosystem. Bivariate correlations of the number of snags with the other field variables showed an average correlation (absolute value) of 0.21 with a maximum value of 0.48 , not including the slightly higher correlation of 0.64 with dead basal area; 
two variables that were not independent of one another. This finding provided further justification for the independent research objectives investigating the detection and mapping of snags as a separate habitat indicator, which could be used in conjunction with a structural complexity index.

\subsubsection{Interpreting the SCI from a Remote Sensing Perspective}

As previously mentioned, the final SCI derived through RDA modelling included five predictor variables, hereby referred to as IM1 to IM5. The variables represented information about crown texture (IM1 and IM2), shadow brightness and texture (IM3 and IM4, respectively), and elevation (IM5). An examination of the biplot scores (Table 8) provided in the CANOCO output (ter Braak and Šmilauer, 2002; Lepš and Šmilauer, 2003) showed the relative importance of each predictor variable in terms of contribution to the SCI as well as the correlation with the axis. The positive correlation of IM1 and IM2 with the SCI reflects the fact that increasing complexity within tree crowns, caused by dead or thin branches and small gaps in the crowns, manifests itself through greater variation in crown reflectance in the imagery.

IM1 had the greatest contribution towards the SCI and, similar to Olthof and King (2000), showed that the variation of a texture measure, as opposed to simply the mean texture, can be effective in detecting variations within tree crowns (correlation between average of GLCM Homogeneity and standard deviation of GLCM Homogeneity was $0.70(\mathrm{p}<0.001)$ suggesting overlapping, but different information). 
Table 8. Biplot scores of RDAl from final RDA model. Scores show relative importance of each predictor variable towards RDAl and the sign of each shows the relative direction compared to RDA1.

\begin{tabular}{clc}
\hline Variable \# & \multicolumn{1}{c}{ Predictor Variable } & $\begin{array}{c}\text { RDA Biplot } \\
\text { Score }\end{array}$ \\
\hline IM1 & $\sigma$ of GLCM Homogeneity (Crowns) & 0.86 \\
IM2 & $\bar{x}$ GLCM Correlation (Crowns) & 0.42 \\
IM3 & $\bar{x}$ NDVI (Shadows) & 0.30 \\
IM4 & $\sigma$ Green reflectance (Shadows) & -0.19 \\
IM5 & Elevation & 0.10 \\
\hline
\end{tabular}

An increase in the mean GLCM Correlation (IM2) in tree crowns was more difficult to interpret, as it is not a very well understood texture measure. Despite these difficulties, it has been successfully used at a variety of scales for biophysical modelling using image variables (Kayitakire et al., 2006; Wunderle et al., 2007). Van der Sanden and Hookman (2005) showed the GLCM Correlation to be a measure of spatial autocorrelation and, in the case of the research presented here, this was found to be true, however, not as straightforward as expected. A bivariate correlation of GLCM Correlation calculated from the entire field plot (i.e. not simply the crowns) was found to have positive correlation with the semivariogram range calculated from the NIR channel $(\mathrm{r}=0.69, \mathrm{p}<0.001)$, which is interpreted as a measure of spatial dependence and often associated with the size of individual objects in high resolution imagery (Lévesque and King, 1999). In fact in these data it was found to be slightly correlated with the average crown diameter measured in the plots $(\mathrm{r}=0.25, \mathrm{p}=0.077)$. NIR-Range was positively correlated with the SCI $(r=0.45, \mathrm{p}<0.001)$, suggesting increased structural complexity 
in the field plot caused greater semivariogram ranges, which was related to objects in the imagery. However, this was contradictory to the expected results. In fact, it seemed that instead of being related to plot crown sizes, the objects that were captured by the NIRRange were the shadows in the plots, as both NIR-Range and plot-based GLCM Correlation were found to be positively correlated with the canopy openness (measured at $6 \mathrm{~m})(\mathrm{r}=0.25, \mathrm{p}=0.081$ and $\mathrm{r}=0.37, \mathrm{p}=0.009$, respectively $)$ and the percent shadow within each plot $(r=0.62, p<0.001$ and $r=0.56, p<0.001$, respectively). This finding suggests that both variables perhaps increased as the canopy gaps and shadow objects increased. It is possible that this relation could be applied within tree crowns as well, where the average Correlation texture from within tree crowns was found to be positively correlated with the SCI, and perhaps this measure was simply representing increasing shadows within the tree crowns, which is thought to be representative of within-crown heterogeneity and therefore increased plot complexity.

As an alternative explanation, GLCM Correlation is also thought to be a useful measure to define borders / transitions as well as identifying patterns (pixel to pixel predictability) within imagery (M. Hall-Beyer, pers. comm., 2008) and therefore indicative of internal transitions between dense leaf area within the crowns and shadow areas and / or exposed wood.

The two predictor variables calculated from within plot shadows were thought to be related to gaps in the canopy as well as shadows caused by variable tree heights within the plots. Increasing structural complexity corresponded with an increase in the NDVI within-shadows (IM3) and a decrease in the variation of green reflectance within- 
shadows (IM4). Higher NDVI in shadows suggested the presence of vegetation within the shadows, which was supported by the presence of $>2 \mathrm{~m}$ understorey vegetation (correlation between $>2 \mathrm{~m}$ understorey vegetation with mean NDVI within shadows was $\mathrm{r}=0.33, \mathrm{p}=0.018)$. Within-shadow NDVI also potentially indicated vertical layering in the plots causing mutual shading, with spectral information still present within the shadows. It must be noted here that since plot shadows do include the shaded side of tree crowns, the within-shadow NDVI may be influenced by the sun angle, with lower sun angles producing longer shadows in the forest. Since the shaded side of crowns could not be separated from between crown shadows, as a result of the sun angle issue, it is possible that NDVI could increase without any understorey in the between crown shadows. Finally, although considered a weak contributor, the presence of understorey vegetation (generally thick maple saplings) potentially caused lower variability in green reflectance within the shadows, however the correlation between the two was not significant $(\mathrm{r}=-0.22,0.130)$.

Finally, elevation (IM5) was positively correlated with increasing structural complexity, although as can be seen in the triplot (Figure 12) it was much more related to the second RDA axis ( $y$ axis in the triplot) than the first $(r=0.73, p<0.001)$. In fact, most of this second axis, which although was not found to be highly significant, was made up mostly by the elevation variable, suggesting that a small portion of structural variance was explainable purely by elevation variations. The inclusion of elevation in the SCI corresponds well to previous research that has shown greater ice storm damage at higher elevations in Gatineau Park (King et al., 2005) as a direct result of wind exposure (Bragg et al., 2003) that created canopy gaps and provided an environment for increased 
understorey and ground vegetation growth. That being said, the second axis may have shown some residual brightness variations in the imagery caused by topographic changes, since the original model included the illumination variable and the final model included elevation. Further, the positive correlation between these two variables $(r=0.40, p=$ 0.004) suggests that they may be somewhat interchangeable in the modelling.

\subsubsection{Assessment of the RDA Model}

This model was initially validated through the second phase of RDA bootstrapping described in Section 4.3.4, which left out ten plots within each iteration for model validation. This validation analysis provided a distribution of \% RMSE (calculated as a percent of the range of RDA 1 values) ranging from $9.8 \%$ to $34.9 \%$, with an average error of $19.9 \%$ (S.D. $=5.1 \%$ ) (Figure 14), providing an idea of the model's accuracy.

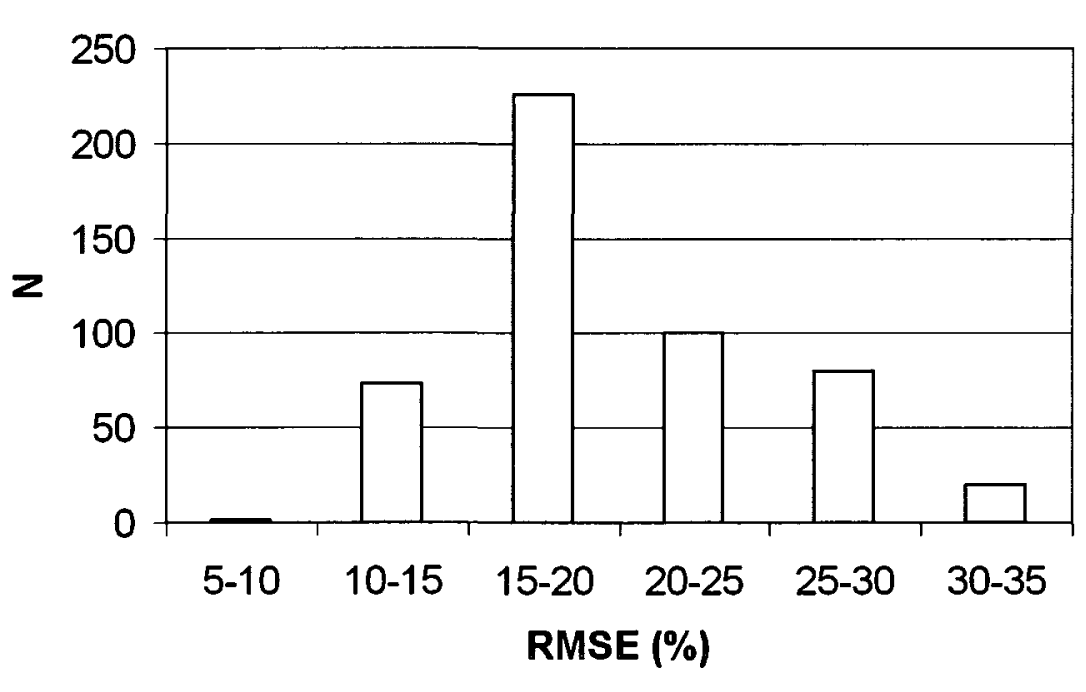

Figure 14. Distribution of RMSE (\%) derived through the second bootstrapping phase for selected 5-variable RDA model. 


\subsubsection{Structural Complexity Mapping and Validation}

The SCI, as represented by RDA1, was a linear combination of predictor variables (Equation 3). This equation was constructed using regression / canonical coefficients for predictor variables (standardized) provided by CANOCO output (ter Braak and Šmilauer, 2002; Leps and Śmilauer, 2003). While one of the biplot scores (from Table 8) was negative (for IM4), which corresponded to a negative correlation with the SCI, the regression / canonical coefficient for this variable was positive (all four others were consistent). This switch in sign was attributed to the fact that in the regression equation (Equation 3) the variance was accounted for by each variable as it was entered into the equation, with each succesive variable contributing explanation of residual variance. The fourth variable entered (IM4), while on its own was negative correlated with the SCI, was entered into the equation with a positive coefficient.

$$
S C I=0.97(I M 1)+0.40(I M 2)+0.21(I M 3)+0.35(I M 4)+0.04(I M 5)
$$

The resultant map of predicted SCI (Figure 15, with close-up example shown in Figure 16) showed patterns of structural complexity across the forest, and included small patches of very high (dark red) and very low (dark green) structural complexity that were different from the surrounding forest (shown on the map as $+/$ - greater than two standard deviations away from the mean). These results, based on field observations, were as expected, and visually, the map corresponded well to general variations seen in the forest in terms of areas of very high and very low structural complexity. With all the predictor layers (i.e. continuous spatial layers representing each of the five predictor variables) 
standardized, the SCI map had standardized values ranging from -2.8 to +2.8 (99\% of the pixels). With the results presented in Figure 13 aside, structural complexity was still considered to be a continuous gradient, and rather than arbitrarily splitting the map into classes representing low, medium and high complexity, the map was simply symbolized using standard deviations, ranging from lowest to highest structural complexity. 


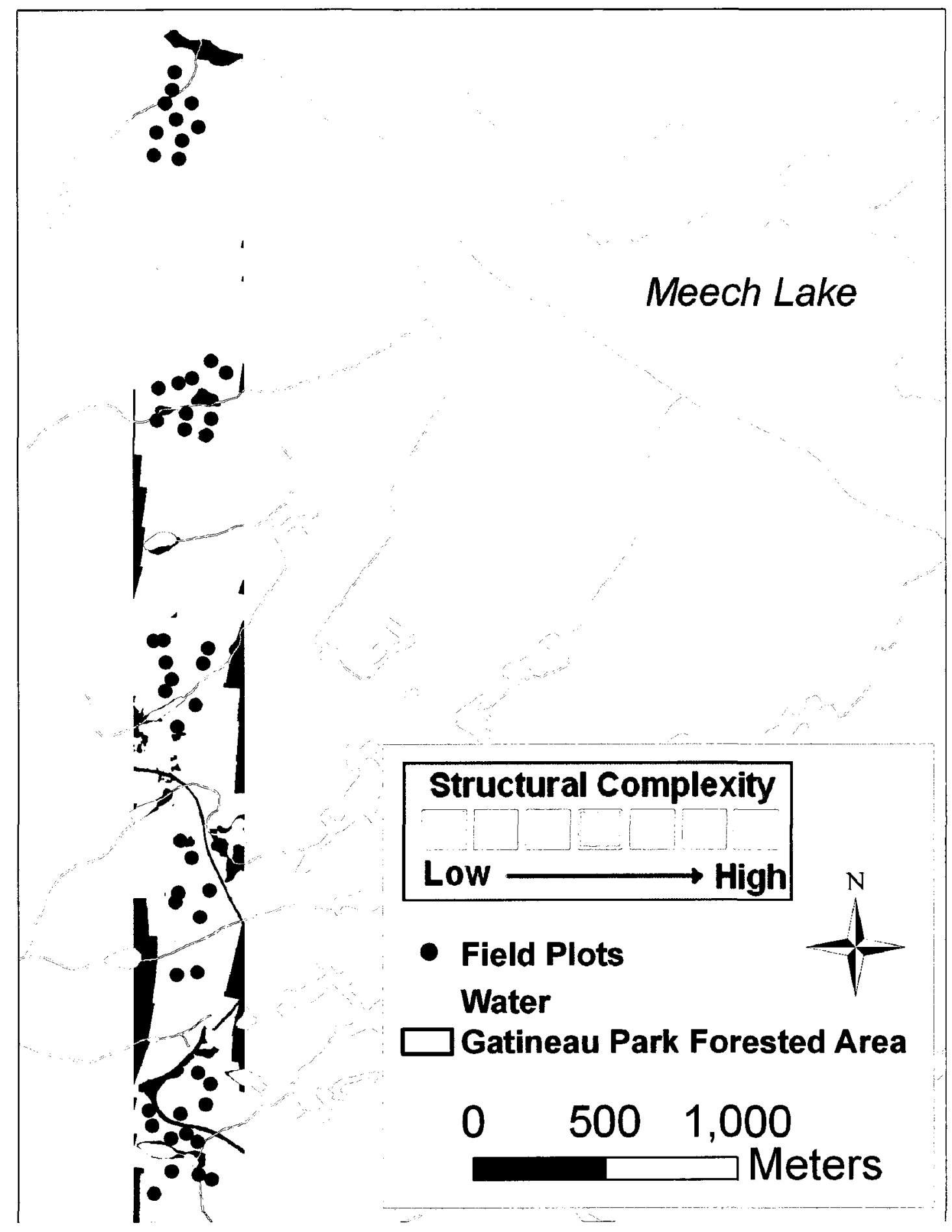

Figure 15. Predicted SCI across the entire study area. The legend for this map, as well as all other SCI maps presented, were created using a standard deviation classification and symbolization. 

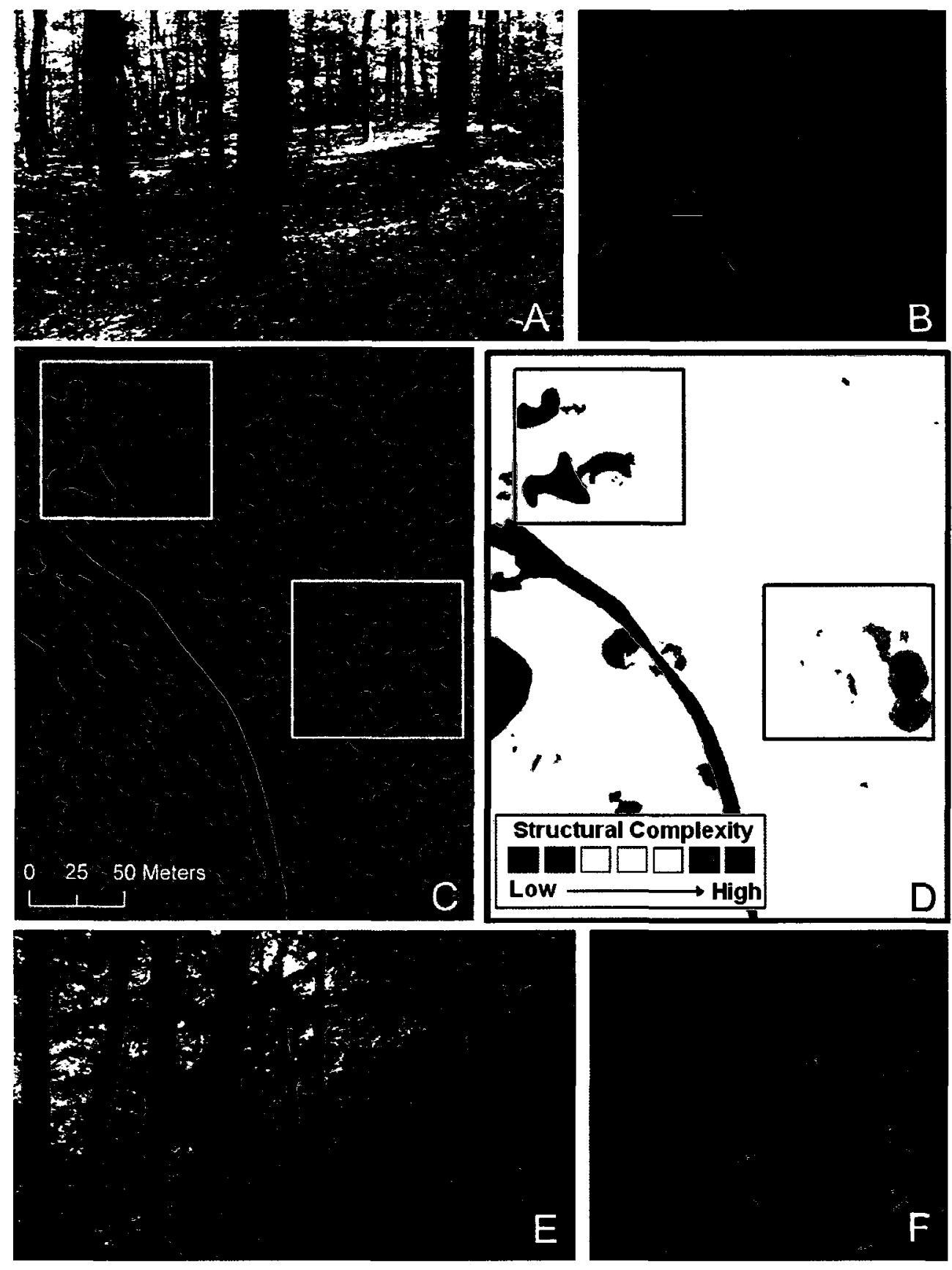

Figure 16. Examples of mapped predicted SCI. Masked roads and water bodies are black. (A) Field photo showing low structural complexity, (B) A low complexity area at full CIR image resolution (as outlined in C and D), (C) Subsection of a CIR image showing an area of relatively low complexity (top left box) and an area of relatively high complexity (middle right box), (D) Map of predicted structural complexity corresponding to the image area in $\mathrm{C},(\mathrm{E})$ Field photo showing high structural complexity, and (F) A high complexity area at full CIR image resolution (as outlined in C and D). 
While the RDA bootstrapping procedure provided validation for the final SCI model $($ RMSE $=19.9 \%)$, the field-based validation provided both independent validation of the model and of the actual mapping procedure, which extended the model from pointbased information to spatially continuous estimates of structural complexity. Of interest, areas of predicted high complexity were more numerous but generally much smaller compared to the areas of predicted low complexity, which resulted in this uneven sample selection.

The results of the field validation (Table 9) showed that the areas predicted by the map as having relatively high structural complexity were well classified with an accuracy of $88.2 \%$ ( 15 out of 17 regions) correct. Of the two areas that were misclassified as high structural complexity, one was found to have a gully running through it that could possibly increase mutual crown shading from tree height variations, thus increasing its predicted structural complexity. The relatively low complexity areas were not as well classified with an accuracy of only $50 \%$ (4 out of 8 areas). Three of the four low complexity areas (notably the three largest), although appearing homogeneous on the SCI map, were assessed in the field as being only partially correct with major changes in complexity levels seen within the GPS boundaries of the areas classified as low complexity (e.g. Figure 17).

Based on the point scoring system (Section 4.3.6), the high complexity regions were still $88.2 \%$ correctly classified ( 15 out of a possible 17 points), while the low complexity regions were in fact $68.8 \%$ correct ( 5.5 out of a possible 8 points). The average accuracy, of $78.5 \%$, was close to the model's accuracy found through 
bootstrapping (80.1\%), although difficult to directly compare since it focused only on the extreme complexity conditions (two classes) and not the typical forest structural conditions (i.e. moderate complexity).

Table 9. Results of field validation for high and low complexity sites, presented following assessment of correct vs. incorrect classification, as well as incorporating partially correct scores of 0.5 .

\begin{tabular}{lcccc}
\hline & \multicolumn{2}{c}{$\begin{array}{c}\text { High Complexity } \\
\text { Regions }\end{array}$} & \multicolumn{2}{c}{$\begin{array}{c}\text { Low Complexity } \\
\text { Regions }\end{array}$} \\
& $\#$ & Points & $\#$ & Points \\
\hline Correctly Classified & 15 & 15 & 4 & 4 \\
Incorrectly Classified & 2 & 0 & 1 & 0 \\
Partially Correctly Classified & 0 & 0 & 3 & 1.5 \\
\hline
\end{tabular}



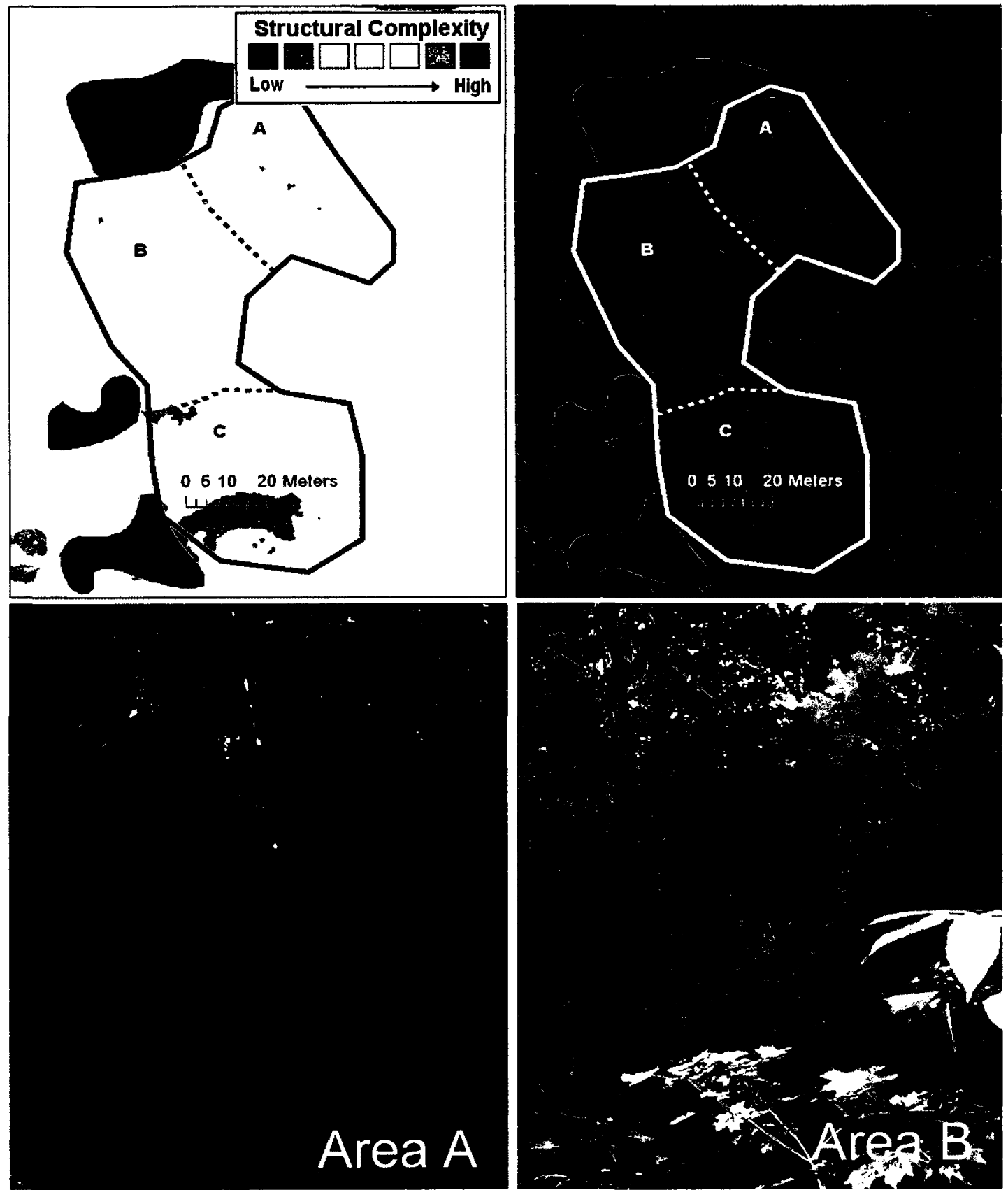

Figure 17. An example of partially correct classification of low complexity area. The area $\left(6096 \mathrm{~m}^{2}\right)$ appeared fairly homogenous in the SCI map, however, while the zones labeled $\mathrm{A}$ and $\mathrm{C}$ were correctly classified as low complexity, the zone labeled $\mathrm{B}$ was incorrect, resulting in an overall assessment of a partial correct classification. The field photos labeled "Area A" and "Area B" correspond with the same areas on the SCI map (A was correctly classified as low complexity and B was incorrectly classified as low complexity). 


\subsubsection{Scalability of RDA Modelling and Mapping}

\subsubsection{RDA Modelling}

Resampling the original airborne imagery from $20 \mathrm{~cm}$ pixels to $60 \mathrm{~cm}$ and $1 \mathrm{~m}$ pixels resulted in an obvious loss of spatial information. Subtle variations and shadows within tree crowns were greatly reduced and almost disappeared in the $1 \mathrm{~m}$ imagery, and individual objects such as exposed wood were almost completely lost.

The first phase of the RDA bootstrapping procedure showed that models produced using variables extracted from $60 \mathrm{~cm}$ and $1 \mathrm{~m}$ imagery explained less of the variance found in the field dataset compared with similar results using the original $20 \mathrm{~cm}$ imagery as was shown in Table 4. Table 10 shows summary statistics calculated for both of these RDA analyses. Interestingly the average adjusted $\mathrm{R}^{2}$ of the models produced using the $60 \mathrm{~cm}$ and $1 \mathrm{~m}$ imagery was 0.28 and 0.25 respectively, which was only slightly lower than the original $20 \mathrm{~cm}$ models (average adjusted $\mathrm{R}^{2}=0.29$ ). The $\%$ RMSE of the models produced with the degraded imagery was slightly higher $(\bar{x}=28.7 \%$ and $30.8 \%$ for $60 \mathrm{~cm}$ and $1 \mathrm{~m}$, respectively) compared to $25.6 \%$ for the $20 \mathrm{~cm}$ model. Also of interest, the average number of predictors included in the models dropped from 3.12 (20 $\mathrm{cm}$ modelling) to $2.60(60 \mathrm{~cm})$ and $2.03(1 \mathrm{~m})$.

As the resolution of the imagery became coarser the importance of full plot information compared to within-feature and object-based information became evident. Table 11 presents the distribution of predictor variable sources / types of information as they were included in the new models, compared to the distribution found in the original $20 \mathrm{~cm}$ analysis. While the original $20 \mathrm{~cm}$ RDA showed within-crown information to be 
Table 10. Summary statistics calculated from models produced from the first phase of RDA bootstrapping using predictor variables extracted from $60 \mathrm{~cm}$ and $1 \mathrm{~m}$ imagery independently.

\begin{tabular}{llccccc}
\hline & & $\begin{array}{c}\text { Adjusted } \\
\mathbf{R}^{2}\end{array}$ & r-Validate & $\begin{array}{c}\text { \# of } \\
\text { Predictors }\end{array}$ & $\begin{array}{c}\% \\
\text { RMSE }\end{array}$ & AIC \\
\hline $\mathbf{6 0} \mathbf{~ c m}$ & Average & 0.28 & 0.69 & 2.60 & 28.7 & 349.2 \\
& Minimum & 0.19 & 0.61 & 1.00 & 19.7 & 346.6 \\
& Maximum & 0.46 & 0.79 & 16.00 & 36.5 & 368.8 \\
& & & & & & \\
$\mathbf{1} \mathbf{~ m}$ & Average & 0.25 & 0.65 & 2.03 & 30.8 & 350.0 \\
& Minimum & 0.07 & 0.36 & 1.00 & 23.3 & 346.9 \\
& Maximum & 0.37 & 0.76 & 10.00 & 51.4 & 360.7 \\
\hline
\end{tabular}

Table 11. Sources / types of predictor variables included in the RDA models produced through bootstrapping the RDA analysis with predictor variables extracted from $20 \mathrm{~cm}$, $60 \mathrm{~cm}$, and $1 \mathrm{~m}$ imagery independently. The top three contributors for each resolution are bolded to highlight major differences.

\begin{tabular}{lccc}
\hline & \multicolumn{3}{c}{ \% of Models } \\
& $\mathbf{2 0} \mathbf{~ c m}$ & $\mathbf{6 0} \mathbf{~ c m}$ & $\mathbf{1 ~} \mathbf{~}$ \\
\hline Within Crown GLCM Texture & $\mathbf{7 0}$ & $\mathbf{2 5}$ & 16 \\
Topographic & $\mathbf{3 2}$ & $\mathbf{2 9}$ & $\mathbf{2 8}$ \\
Semivariogram Range and Sill & $\mathbf{2 8}$ & 6 & 14 \\
Within Crown Spectral & 22 & 12 & 2 \\
Within Shadow $1^{\text {st }}$ Order Spatial & 21 & 2 & 3 \\
Object-based & 19 & 3 & 1 \\
Within Shadow GLCM Texture & 18 & 5 & 14 \\
Full Plot GLCM Texture & 11 & $\mathbf{7 3}$ & 16 \\
Subpixel Fractions & 10 & 23 & $\mathbf{4 2}$ \\
Within Shadow Spectral & 8 & 1 & 1 \\
Within Crown $1^{\text {st }}$ Order Spatial & 2 & 4 & 2 \\
Full Plot 15t $^{\text {Order Spatial }}$ & 1 & 7 & $\mathbf{4 4}$ \\
Full Plot Spectral & 1 & 18 & 6 \\
\hline
\end{tabular}


extremely important predictors (within GLCM textures included in $70 \%$ of the models and within-crown spectral information included in $22 \%$ ), the reduced within-crown information found in the degraded imagery was evident. The inclusion of within-crown information was greatly reduced in the $60 \mathrm{~cm}$ RDA (GLCM texture $25 \%$ and spectral 12 $\%$ ) as well as the $1 \mathrm{~m} \mathrm{RDA}$ (GLCM texture $16 \%$ and spectral $2 \%$ ). Full plot spatial information contributed to $73 \%$ of models produced with $60 \mathrm{~cm}$ data, and interestingly subpixel fractions were found to be extremely important for models produced from the 1 $\mathrm{m}$ data, contributing to $42 \%$ of the models, suggesting that information from the mixing of pixels was not completely lost after all. It is worth noting that the contribution of topographic information remained stable as was expected given that the DEM was not affected by the change in resolution.

The best models that were created from the RDA using $60 \mathrm{~cm}$ data had between six and nine predictor variables. Of the next best models, which included only four or five variables, several had variance inflation issues. The final model that was selected included four predictor variables and its first RDA axis (the second axis was not significant) had an adjusted $\mathrm{R}^{2}$ of $0.32(\mathrm{p}=0.002)$, providing only $3 \%$ less explanation of the variance in the field data compared to the original RDAl produced from the $20 \mathrm{~cm}$ imagery. A bootstrapping procedure carried out for this model showed the model to have a \% RMSE ranging from $11.9 \%$ to $46.5 \%$, with an average error of $24.9 \%$ (S.D. $=6.5$ $\%$ ), which was approximately $5 \%$ higher than the original $20 \mathrm{~cm}$ model. The four predictor variables included in this model were: (1) average NDVI for the entire plot, (2) variation of unmixed sunlit fraction measured across the entire plot, (3) average GLCM 
Contrast within-crowns, and (4) elevation. The Structural Complexity Index $\left(\mathrm{SCI}_{60 \mathrm{~cm}}\right)$, or first RDA axis, of this model was found to be correlated with the original $\mathrm{SCI}_{20 \mathrm{~cm}}$, with a correlation coefficient of $0.84(\mathrm{p}<0.001)$. Similarly, the correlations between $\mathrm{SCI}_{60 \mathrm{~cm}}$ and the field variables only differed on average by 0.066 (maximum $=0.132$ ) from the corresponding correlations found with $\mathrm{SCI}_{20 \mathrm{~cm}}$ and the field variables (previously shown in Table 6).

Looking at the results of the RDA carried out using the $1 \mathrm{~m}$ data, all of the best models included only three or four predictor variables. The selected model, which was the first with no variance inflation issues, contained three predictor variables: (1) variation in green reflectance across the plot, (2) average GLCM Contrast across the plot, and (3) elevation. This model's first RDA axis (again the only significant one) had an adjusted $\mathrm{R}^{2}$ of $0.30(\mathrm{p}=0.002)$ with an RMSE ranging from $13.5 \%$ to $49.8 \%(\overline{\mathrm{x}}=28.4$ $\%$, S.D. $=8.1 \%$, again showing slightly less explanation of the variance in field data with a slightly higher percent error. Again, the difference in correlations between $\mathrm{SCI}_{1 \mathrm{~m}}$ and the field variables compared to the corresponding correlations using $\mathbf{S C I}_{20 \mathrm{~cm}}$ were quite small $(\overline{\mathrm{x}}=0.077$, maximum $=0.15)$.

\subsubsection{Mapping Structural Complexity}

The linear combination of predictor variables, each multiplied by the corresponding regression / canonical coefficients provided by CANOCO for each of the models, produced maps of predicted SCI at $60 \mathrm{~cm}$ and $1 \mathrm{~m}$ resolutions. The two maps produced from degraded imagery were directly comparable to the original SCI map produced using $20 \mathrm{~cm}$ predictor variables. Figure 17 shows two example areas taken from 
the study area in order to show differences seen in the mapped SCIs. Many areas across the maps corresponded very well (Figure 18, Example 1), with patches of relatively high and low structural complexity clearly visible in the $60 \mathrm{~cm}$ and $1 \mathrm{~m}$ maps that were visible on the original $20 \mathrm{~cm}$ map. Patches tended to be less distinct in the coarser resolution maps. There were, however, many areas in the maps where significant differences in the patches of high and low complexity were visible (Figure 18, Example 2), however, without further field exploration of these specific areas on the maps it would be impossible to speculate what these changes were, or if one map was in fact more accurate that another.

In order to attempt to compare the maps to each other quantitatively, a set of twohundred and fifty random plots ( $11.3 \mathrm{~m}$ radius circles) were created across the maps and the average SCI value within the plots were calculated. At the plot scale, and independent of the actual plots used to create the RDA model, the two lower resolution maps were found to be highly correlated with each other $(r=0.90, p<0.001)$ while the $60 \mathrm{~cm}$ and 1 $\mathrm{m}$ maps were found to be less correlated with the higher resolution map $(r=0.66, p<$ 0.001 and $r=0.65, p<0.001$ respectively).

Finally, a comparison of the three maps based on the high $(n=17)$ and low $(n=$ 8) complexity regions that were used for independent field validation of the extreme conditions found on the original SCI map $(20 \mathrm{~cm})$ was carried out, which showed some interesting differences. While the original map accuracy for high and low complexity regions was $88.2 \%$ and $68.8 \%$, respectively, the other two maps were found to have lower accuracies. The $60 \mathrm{~cm}$ map showed high and low complexity regions to be $70.6 \%$ 
(12 pts) and $56.3 \%$ (4.5 pts) accurate, while the $1 \mathrm{~m}$ map showed accuracies of only 61.8 $\%$ and $37.5 \%$ for high and low complexity regions respectively.

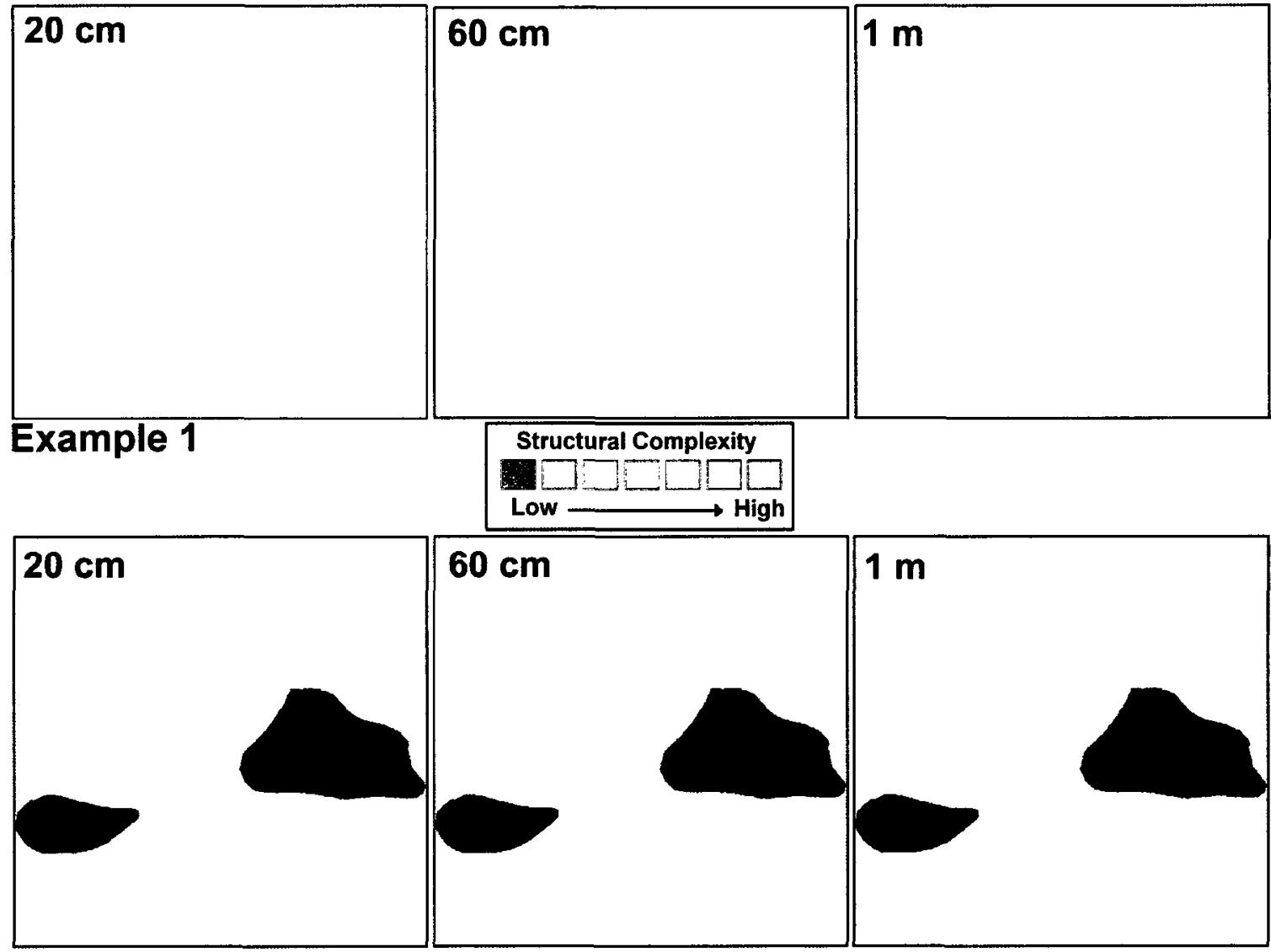

\section{Example 2}

Figure 18. Two sample regions (each $250 \mathrm{~m} \times 250 \mathrm{~m}$ ) extracted from the three different resolution maps of the predicted SCI. The two examples show an area that corresponded well between the different resolutions (Example 1) as well as one that seemed to show significant differences (Example 2) between the lower resolution maps and the original $20 \mathrm{~cm} \mathrm{SCI}$ map. Masked water bodies appear as black. 


\subsection{Modelling and Mapping Dead Wood}

\subsubsection{Direct Detection of Dead Wood}

Each of the three methods used to detect and delineate wood within the imagery produced slightly different maps. Figure 19 shows an example of two large snags classified by the three methods to illustrate the delineations at the pixel level, with fully intact live tree crowns shown in comparison along with visible shadows / canopy gaps. The generalization procedure that was carried out cleaned up the maps, and, as shown in an example of one of the three classification methods (Figure 20), combined dead wood objects that were obviously part of the same snag, as well as removing the pixelated nature of the objects.

As expected, since it was the most basic method, the ISODATA clustering resulted in less than half of the total area classified as wood compared to the other two methods (last column, Table 12). The "hard" nature of the algorithm resulted in clusters that were composed solely of pure wood pixels. The transitions, or mixed pixels between these wood objects and adjacent vegetation or shadow in the imagery were inconsistently classified and could not be easily associated with the pixels that were clearly wood. Even though the imagery was very high resolution $(20 \mathrm{~cm}$ pixel size $)$, small objects such as branches resulted in mixed pixels that were not well classified with the ISODATA algorithm. 


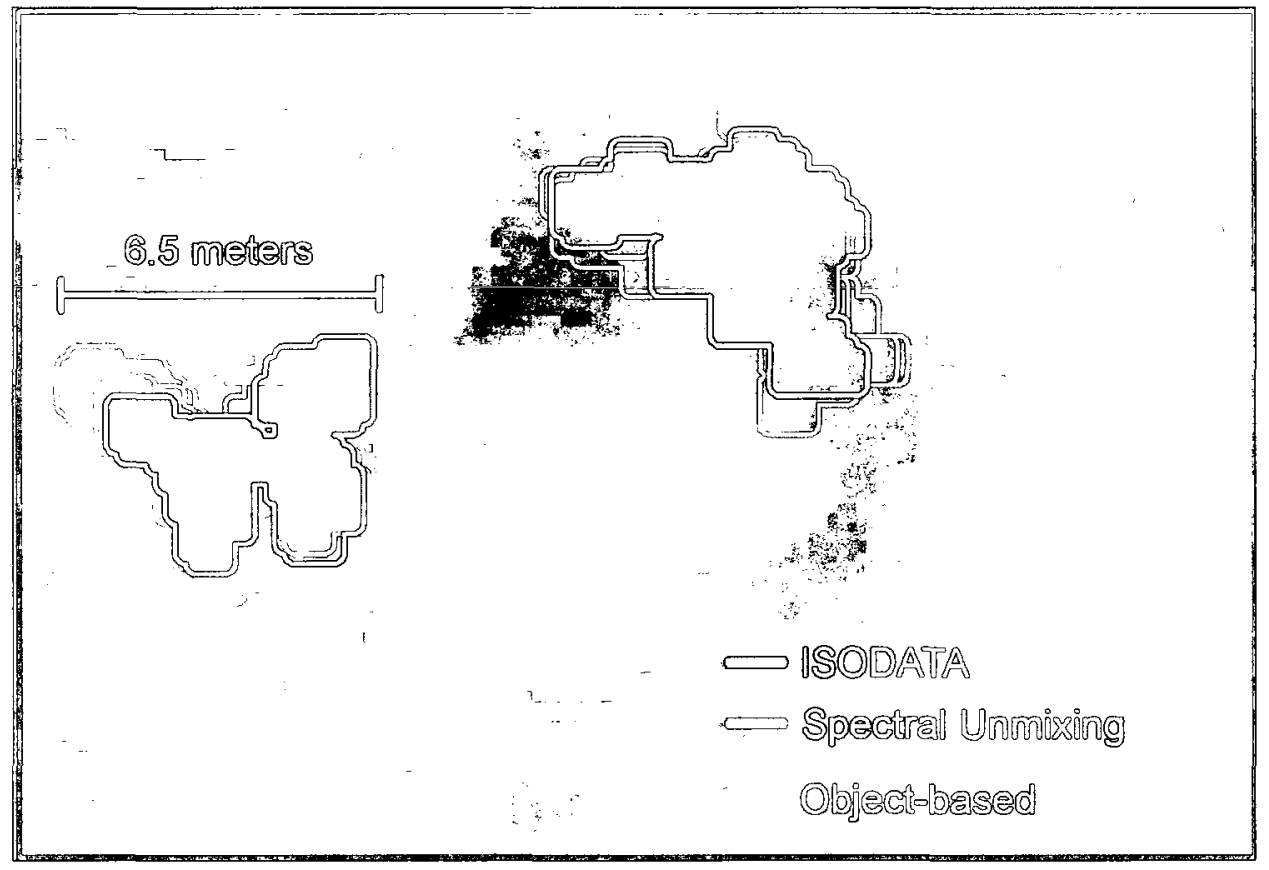

Fignure 19. A comparison of the three detection and delineation methods for two large snags shown with the $\mathbb{C I R}$ imagery in the background.

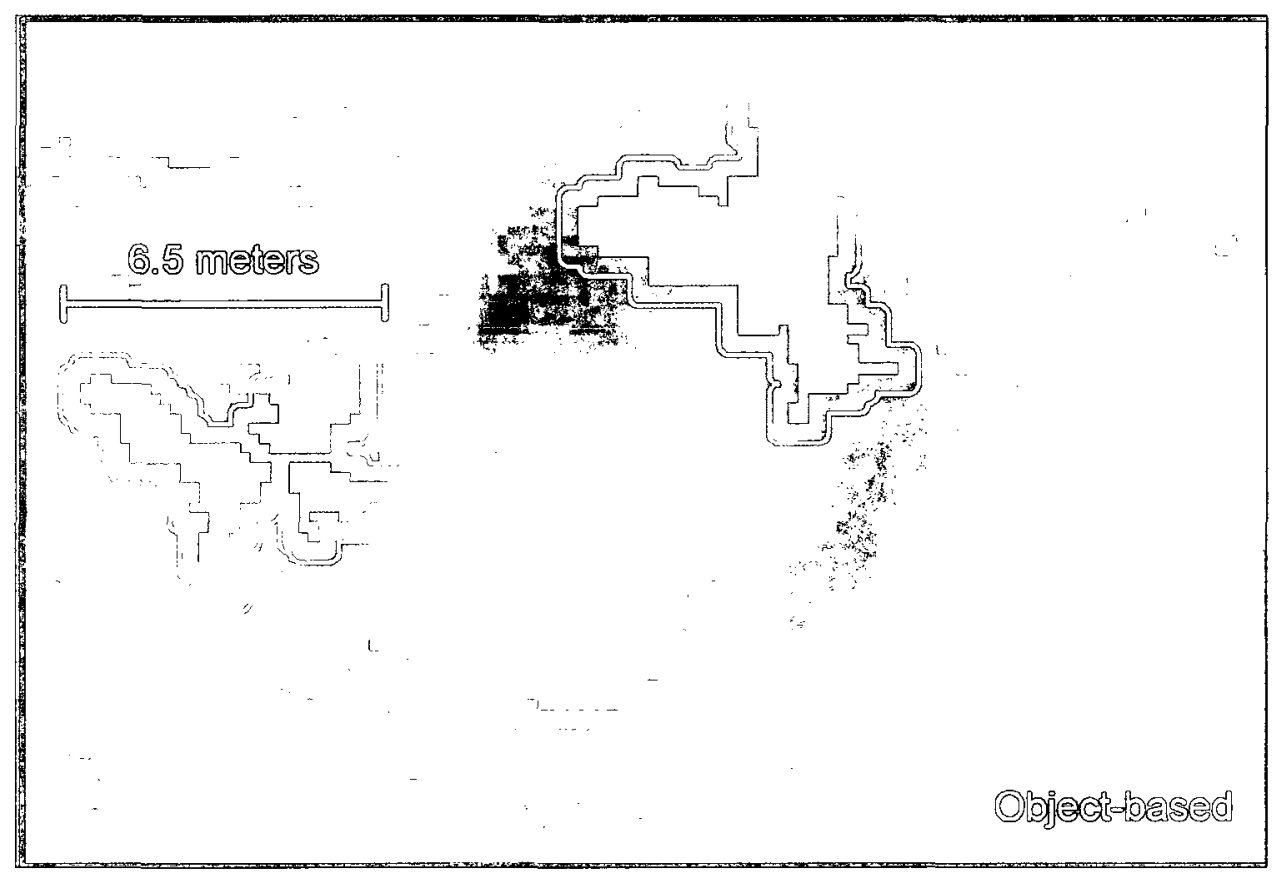

Figure 20. An example of the processing carried out to group adjacent wood objects as well as generalize their boundaries. Solid objects shown were grouped and buffered to created objects with boundaries defined by the lines. For visualization purposes only the object-based method is shown as an example, however the same process was carried out for the other two methods as well. 
Table 12. Wood object statistics as derived using the three different classification methods and by intersecting these three preliminary maps into a hybrid classification.

\begin{tabular}{lcccccc}
\hline & $\mathbf{N}$ & $\begin{array}{c}\text { Minimum } \\
\left(\mathbf{m}^{2}\right)\end{array}$ & $\begin{array}{c}\text { Maximum } \\
\left(\mathbf{m}^{2}\right)\end{array}$ & $\begin{array}{c}\text { Average } \\
\left(\mathbf{m}^{2}\right)\end{array}$ & $\begin{array}{c}\text { Standard } \\
\text { Deviation } \\
\left(\mathbf{m}^{2}\right)\end{array}$ & $\begin{array}{c}\text { Sum } \\
\left(\mathbf{m}^{2}\right)\end{array}$ \\
\hline $\begin{array}{l}\text { ISODATA } \\
\text { Clustering }\end{array}$ & 4972 & 1.12 & 54.93 & 3.47 & 3.70 & 17250 \\
$\begin{array}{l}\text { Spectral } \\
\text { Unmixing }\end{array}$ & 11464 & 1.09 & 86.78 & 3.61 & 4.32 & 41370 \\
$\begin{array}{l}\text { Object- } \\
\text { based }\end{array}$ & 5977 & 1.36 & 84.06 & 6.16 & 6.29 & 36818 \\
$\begin{array}{l}\text { Final } \\
\text { Hybrid Map }\end{array}$ & 6344 & 0.08 & 82.52 & 4.41 & 4.98 & 27969 \\
\hline
\end{tabular}

This result was also evident in the maximum size of wood objects, which, in the ISODATA map was only $54.93 \mathrm{~m}^{2}$, compared to $86.78 \mathrm{~m}^{2}$ and $84.06 \mathrm{~m}^{2}$ for the spectral unmixing and object-based methods, respectively. Interestingly, the ISODATA clustering had a similar number of objects as the object-based method (4972 compared to 5977 ). This was expected since the object segmentation and classification was carried out using visual interpretation to define the object boundaries as well as the selected membership value threshold. For this reason these two methods detected the most identifiable wood objects. On the other hand, the spectral unmixing method identified many smaller objects (resulting in approximately twice as many objects detected) within what appeared to be thick and healthy tree crowns as well as within dark shadow areas (Figure 21).

The final map of wood objects (an example sub-area is shown in Figure 22) contained only those objects with the highest probability of actually being wood objects 
in the forest. All of these objects, given their image characteristics, were called wood objects, with very few other features, such as rocks, potentially detected and included. The assessment of probability was based on the assumption that if at least two of the three methods detected a particular pixel then the chance of it actually being a wood pixel rather than a false positive was much higher. Based on this, many of the small objects previously discussed that were found by the unmixing method ended up being discarded, with the final map having only 6344 objects, ranging from $0.08 \mathrm{~m}^{2}$ (only 1.5 pixels) to $82.52 \mathrm{~m}^{2}$ (a dead crown of approximately $10 \mathrm{~m}$ diameter). While $96 \%$ of the objects detected using the ISODATA method and $88 \%$ of those detected using the object-based method matched in location (not necessarily in size) with the final objects, only $55 \%$ of the objects detected using spectral unmixing were included in the final classified map. Those objects not included in the final map $(n=5212)$ had areas ranging from $1.08-$ $18.24 \mathrm{~m}^{2}\left(\overline{\mathrm{x}}=2.06 \mathrm{~m}^{2}\right.$, S.D. $\left.=1.24 \mathrm{~m}^{2}\right)$. 


\section{๑

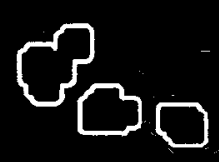

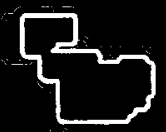

$\square$

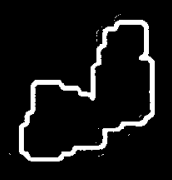

$\square$

\section{ISODATA}

Spectral Unmixing

Object-based

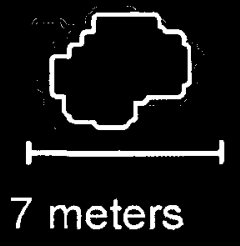

Figure 21. A typical example of detected wood objects shown with the CIR imagery in the background. Spectral unmixing detected many small objects that were not detected by the other two methods. 


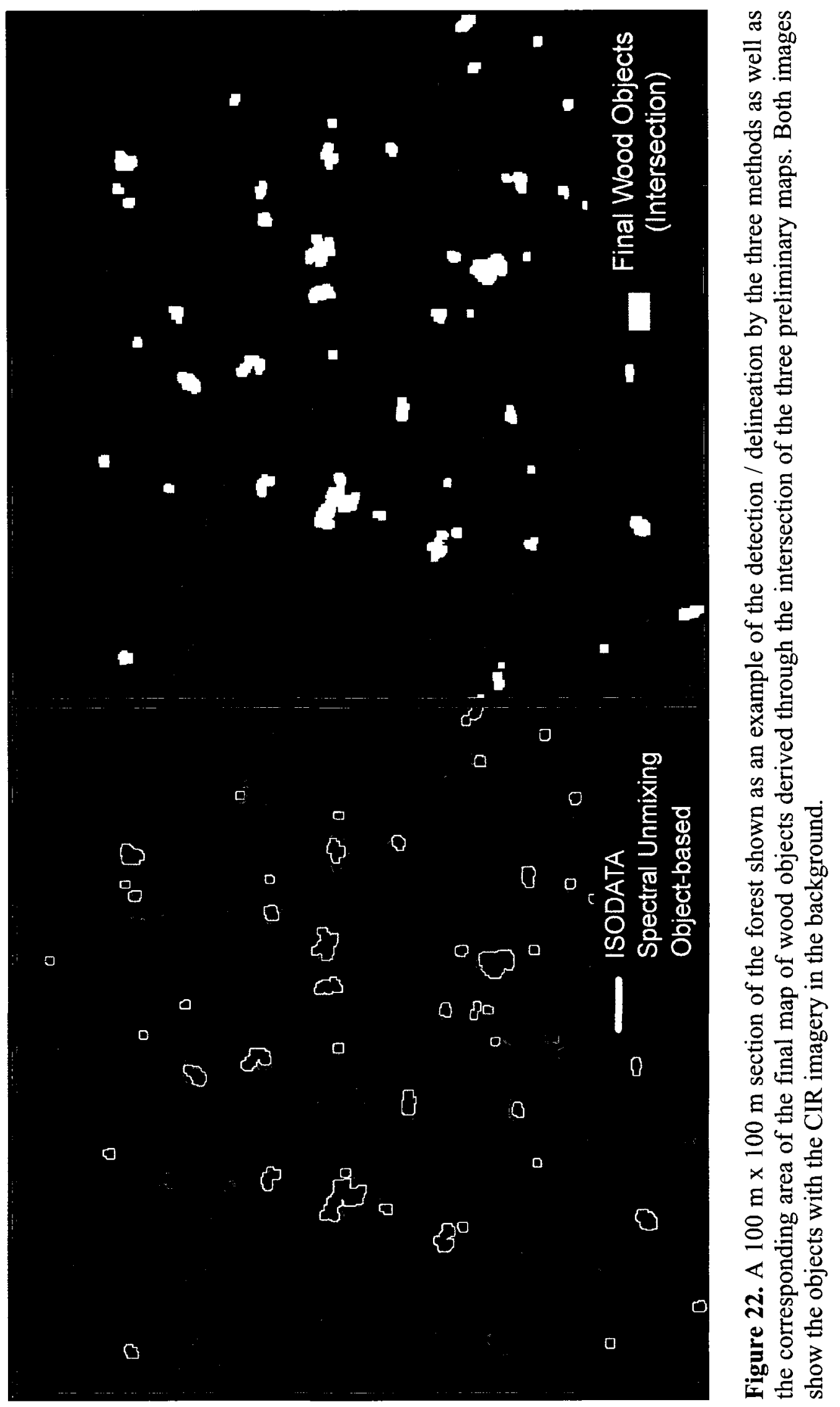


While the spectral unmixing method detected approximately twice as many wood objects as the other two methods, most were very small and impossible to validate in the field. Increasing the fraction threshold to greater than $50 \%$ would reduce the number of wood objects detected, however, selection of a threshold is arbitrary. Testing of thresholds from $30-70 \%$ clearly showed an exponential decrease in the number of wood objects detected with increasing thresholds $\left(\mathrm{R}^{2}\right.$ of trendline $\left.=0.99\right)$. The threshold of $50 \%$ was selected because of its ability to detect the significant and obvious wood objects based on tests done using manual interpretation. Thresholds of less than $50 \%$ included too many objects that were interpreted as false positives and too many pixels around the edges of known dead wood objects (examples shown in Figure 23). As well, at thresholds less than $50 \%$, many single dead wood objects were represented by multiple image objects (examples shown in Figure 23).

Maps of dead wood created using the spectral unmixing method may show more subtle details than those produced from the per pixel methods but it is doubtful that all the small wood objects detected represent significant habitat or dead wood of importance to inventories (e.g. for carbon modelling, forest health assessment, etc.). Given that this research had the goal of producing an accurate map of the relative dead wood spatial distribution across the forest, the dead crowns and large dead branches were of most interest with relatively little interest in the small dead branches within essentially healthy live crowns. As well, from a validation point of view, without a close-up view of the top of the canopy (e.g., using a cherry-picker or ladder (Barker and Pinard, 2001; Moorthy et al., 2007)), these objects would not have been visible on the ground beneath the canopy. 


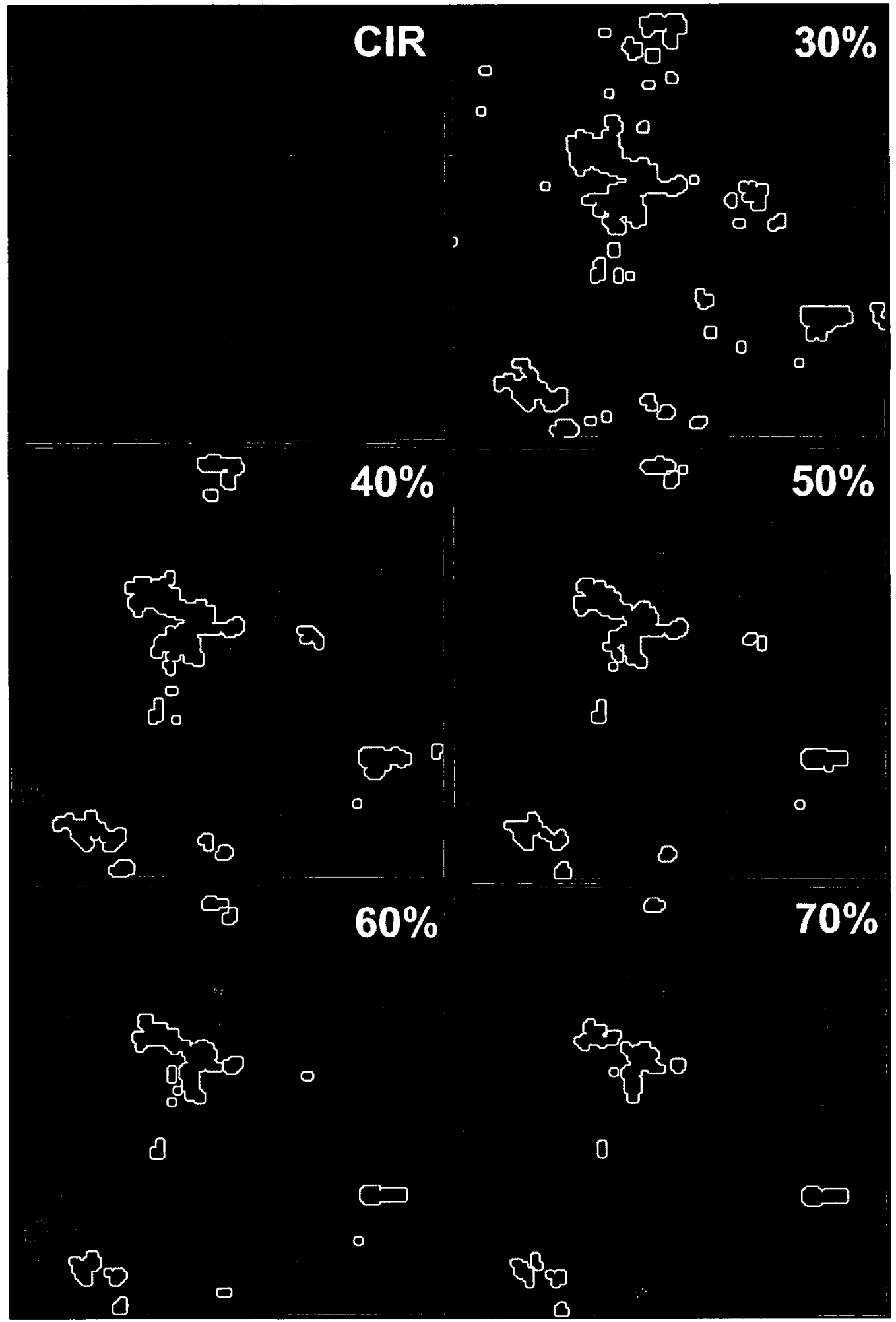

Figure 23. An example $30 \mathrm{~m} \times 30 \mathrm{~m}$ sample area showing results of testing to determine a suitable threshold for 'hardening' of the unmixed fraction map. Dead wood object numbers and sizes vary with the thresholds shown (30\% to $70 \%)$. 


\subsubsection{Dead Wood Map Validation}

Of the fifty detected wood objects that were used for validation, forty-one were correctly identified in the field as being snags, resulting in an accuracy of $82 \%$. Of the thirty control point validation locations, where no wood object was found in the imagery, twenty-one were correct, resulting in an accuracy of $70 \%$. These control sites, which were selected to be at least $10 \mathrm{~m}$ away from a detected wood object in the imagery, were in fact on average $20.1 \mathrm{~m}$ (S.D. $=3.6 \mathrm{~m}$ ) away from any wood object in the field. Accordingly, there should not have been any confusion regarding the location of the control site.

\subsubsection{Assessment of Errors of Commission}

The results of directly detecting dead wood across the study area were thought to be quite successful overall, with dead wood objects being accurately detected $82 \%$ of the time, and control sites accurate $70 \%$ of the time. Further investigation based on the field notes recorded at each of the eighty locations ( 50 validation wood objects and 30 control sites) revealed interesting information that aided explanation of the results and improved the mapping accuracies. Of the nine validation sites where a wood object was detected in the imagery yet not in the field (i.e. incorrect detection of a wood object) two were described in the field notes as having a small dead crown in the vicinity that was not thought to be visible from the air. The surface areas of these dead trees, as measured in the imagery, were $2.95 \mathrm{~m}^{2}$ and $4.14 \mathrm{~m}^{2}$, which were close to the minimum size of the fifty validation wood objects (range of $1.37 \mathrm{~m}^{2}-27.10 \mathrm{~m}^{2}$ ). Three additional sites initially assessed as incorrect had been noted in the field to have some dead branches and 
thin crowns in the canopy that were not expected to be detectable. However, these dead branches were probably more visible from above and detected in the imagery. Given these explanations, the actual accuracy of the wood objects could be increased from $82 \%$ to $92 \%$. This adjusted percentage accommodates for the five locations where dead wood that was detected in the imagery was actually found in the forest. The four remaining validation wood objects that were found to be incorrect had no significant dead wood in the vicinity. Three of these four errors, when examined in the imagery, were not obvious dead wood but rather appeared to be thin crowns that were not visible from the observer's position on the forest floor. Portions of branches in these crowns must have been exposed to the sensor view. The fourth location was actually one of the largest wood objects found, with an area of $21.96 \mathrm{~m}^{2}$. Close examination of the imagery showed it to be above the surrounding overstorey canopy, and, as a result of shorter understorey trees, that are partially visible within a shadow in the imagery filling in the adjacent canopy gap (as seen in Figure 24), this dead crown was not visible from the ground. In other words, the image detection procedure was correct while the field validation was incorrect, increasing the overall accuracy to 47 of 50 , or $94 \%$. 


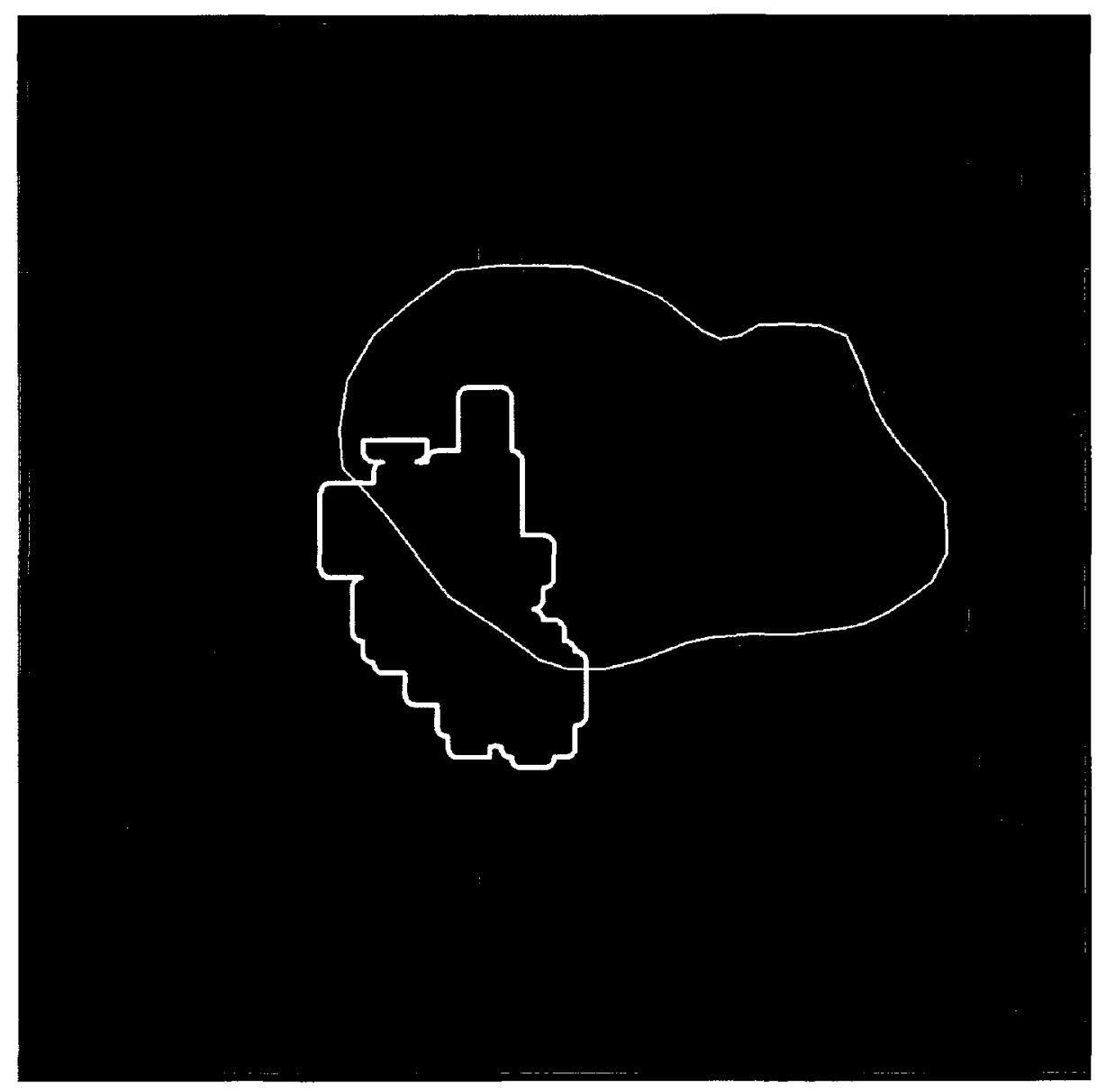

Figure 24. A large dead wood object delineated in the imagery (white outline) that was not seen during field validation. The adjacent and overlapping area of the imagery that is outlined in yellow appears to be a live tree crown seen within a shadow in the canopy.

\subsubsection{Assessment of Errors of Omission}

An examination into the erroneous control sites $(n=9)$, where a wood object was not detected in the imagery but one was found in the field also showed some interesting results. Of these nine control sites two had significant "pole" snags present, i.e. snags with no remaining intact crown or branches. This suggested that such snags were realistically not visible or detectable in the airborne imagery, most likely a result of 
camera view angles leaving the snag blocked from view by surrounding live crowns. Similarly, half-dead crowns were found at four of the other erroneous control sites, again seemingly a result of camera view angles. When examining the imagery more closely at these locations there was no sign of any dead wood that might have been missed by the algorithms. The remaining three erroneous control sites were truly errors of omission. These sites contained, as discovered and measured in the field, significant snags (two of them had dead crowns greater than $5 \mathrm{~m}$ in diameter as estimated from the ground) that were not visible in the imagery. These errors of omission were also potentially due to view angle and differential tree heights in the forest. This result suggests that potentially $10 \%$ of the forested area where no dead wood was detected could contain significant snags, in other words areas with no dead wood were found to be $90 \%$ accurate.

\subsubsection{Mapping Dead Wood Spatial Distribution}

The map of dead wood spatial distribution (Figure 24) across the study area was patchy, reflecting the non-uniform distribution of dead trees that would be expected across a forest (Ducey et al., 2002; Kenning et al., 2005). The relative dead wood density was compared to the actual number of snags that had been counted in the field within the fifty field plots. A correlation of $0.53(\mathrm{p}<0.001)$ was found, however this relationship was clearly controlled by two field plots with a much greater number of snags (12 and 19) compared to the average of $4.9($ S.D. $=3.41)$ found across the fifty plots. Therefore, while the relation was not a strong linear one, these findings suggest that this type of mapping could be used to identify areas in the forest that have large numbers of snags (e.g. as in the lower left portion of Figure 25). 


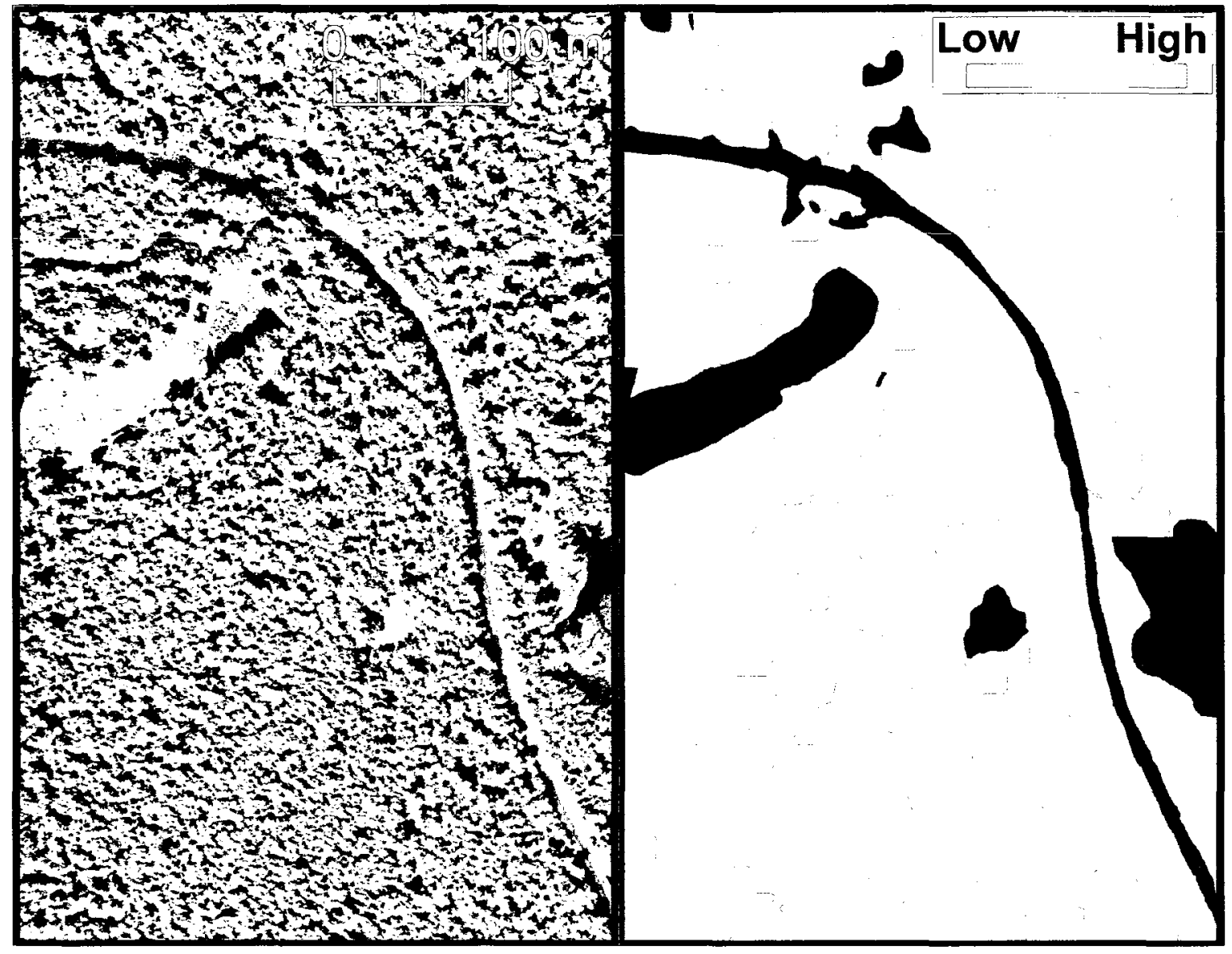

Figure 25. An example subsection of the map of relative dead wood spatial distribution found across the forest as derived from the CIR imagery, shown for the corresponding area. Roads and water bodies were masked out and appear black on the map.

\subsubsection{Indirect Modelling of Dead Wood}

Field measurements in the fifty plots showed a range in the amount of dead wood (Table 13), where the number of snags showed no relationship with both the number of pieces of CWD $(r=-0.01, p=0.961)$ and the volume of coarse woody debris (CWD) (r $=-0.05, p=0.737)$. In contrast, the two measures of CWD were found to be highly correlated $(\mathrm{r}=0.92, \mathrm{p}<0.001)$. 
Table 13. Dead wood statistics as measured in the fifty field plots.

\begin{tabular}{|c|c|c|c|c|}
\hline & Average & $\begin{array}{l}\text { Standard } \\
\text { Deviation }\end{array}$ & Minimum & Maximum \\
\hline Number of Snags & 4.88 & 3.41 & 1 & 19 \\
\hline $\begin{array}{l}\text { Number of Pieces of } \\
\text { Coarse Woody Debris }\end{array}$ & 12.14 & 6.86 & 1 & 30 \\
\hline $\begin{array}{l}\text { Coarse Woody Debris } \\
\text { Volume } \\
\left(\mathrm{m}^{3} / \mathrm{ha}\right)\end{array}$ & 63.24 & 47.05 & 3.94 & 199.05 \\
\hline
\end{tabular}

The best regression model for CWD volume (Table 14) was highly significant but only explained $30.3 \%$ of the variance and had a standard error of $39.27 \mathrm{~m}^{3} /$ ha, or 62.1 $\%$ of the mean volume of all plots. The regression model derived in this case study for the number of pieces of CWD included similar variables but was not as strong as the one produced for CWD volume.

The CWD model was difficult to interpret. However, the main predictor variable, which explained more than half of the variance, suggested that plots with fewer canopy objects had increasing amounts of CWD. The number of canopy objects, derived using a tree delineation algorithm (Pouliot et al., 2005), was significantly positively correlated with the number of overstorey trees $(r=0.54, p<0.001)$. This is potentially indicative of plots that suffered from recent tree deaths, which contributed to the CWD that was found. However, the number of snags was not correlated with the CWD volume $(r=-0.05, p=$ 
Table 14. Regression results for indirect modelling of coarse woody debris volume and the number of snags in fifty plots across the forest. Both models were found to be highly significant $(p<0.001)$.

\begin{tabular}{|c|c|c|c|c|c|c|}
\hline $\begin{array}{c}\text { Dependent } \\
\text { Variable }\end{array}$ & $\mathbf{R}^{2}$ & $\begin{array}{l}\text { Adjusted } \\
\mathbf{R}^{\mathbf{2}}\end{array}$ & SE & $\begin{array}{c}\text { Independent } \\
\text { Variables } \\
\text { (Contribution of } \\
\text { variables } \\
\text { shown by }+l-\text { ) }\end{array}$ & $\begin{array}{l}\text { Partial } \\
\mathbf{R}^{2}\end{array}$ & p \\
\hline \multirow{3}{*}{$\begin{array}{l}\text { Coarse } \\
\text { Woody } \\
\text { Debris } \\
\text { Volume } \\
\left(\mathrm{m}^{3} / \mathrm{ha}\right)\end{array}$} & \multirow{3}{*}{0.35} & \multirow{3}{*}{0.30} & \multirow{3}{*}{39.27} & $\begin{array}{l}\text { - \# of Canopy } \\
\text { Objects }\end{array}$ & 0.21 & $<0.001$ \\
\hline & & & & $\begin{array}{l}\text { - Variation of Area } \\
\text { of Canopy } \\
\text { Objects }\end{array}$ & 0.07 & 0.021 \\
\hline & & & & $+\begin{array}{l}\text { Average NIR } \\
\text { Crowns }\end{array}$ & 0.06 & 0.040 \\
\hline \multirow{2}{*}{$\begin{array}{l}\text { Number of } \\
\text { Snags }\end{array}$} & \multirow{2}{*}{0.36} & \multirow{2}{*}{0.33} & \multirow{2}{*}{2.79} & + Total Area Wood & 0.29 & $<0.001$ \\
\hline & & & & $\begin{array}{l}\text { - \# of Canopy } \\
\text { Objects }\end{array}$ & 0.07 & 0.024 \\
\hline
\end{tabular}

0.737), which suggested that there may have been a time lag between when the data was collected and when the trees died. Interestingly, the third predictor variable included in the model showed increasing within-crown NIR reflectance related to increasing amounts of CWD. This finding supported the idea that the CWD originated from historical fallen trees rather than from partially damaged tree crowns that would have exhibited lower average NIR reflectance. The trees had most likely recovered resulting in higher withincrown NIR reflectance. 
The best model of the number of snags per plot explained slightly more variance than the CWD model (33.2\%) (Table 13), however this model still had a very high error ( $\mathrm{SE}=2.79$ or $57.2 \%$ of the mean number found in all plots). This model was more easily explained. It included only two predictor variables, with the first (i.e. the amount of dead wood found through classification (Section 4.4.3)) contributing $79 \%$ of the explained variance. The other variable included in the model was also an object-based one, as opposed to one reflecting spectral or spatial information in the imagery. The model showed that as the number of canopy objects found in the imagery decreased, the number of snags in the forest increased. This relationship was identical to the one shown in the regression model for CWD, yet, similarly to the results previously discussed for CWD it was difficult to interpret because the number of snags in the plots was uncorrelated with the number of overstorey trees $(r=0.096, p=0.506)$.

\subsection{Combining Indicators}

The two indicators that were derived from the airborne imagery, namely the SCI and the map of dead wood distribution, were found to be completely uncorrelated with one another on a pixel by pixel basis. This was not a surprise for two reasons. First of all, the number of pixels representing snags within each plot was very small and therefore probably not well represented in the plot-based image variables. As well, this discrepancy could potentially be attributed to a temporal element of the forest's structure. Nine years prior to the measurement of field data the forest suffered significant, although patchy, damage from an ice storm. This storm killed many trees that presently still remain standing, however, the surrounding vegetation structure has changed significantly since 
the storm creating a disconnect between the presence of snags and many of the other variables, and as a result snags did not appear to be a main contributor, although certainly important structures, to the SCI. The number of snags was not found to be well correlated with other field variables, and as a result did not fit very well within the RDA model. There is no doubt that snags are potentially a very useful indicator of habitat and biodiversity levels within the forest, and as a result, having multiple indicators could potentially enhance mapping and monitoring efforts. Further this justifies the need for directly detecting spatially rare but important habitat indicators.

The two mapped indicators could be combined in order to attempt to fine tune the results of the SCI. Complexity should increase with an increasing number of dead trees and therefore the mapped spatial distribution of dead trees could be used to modify the SCI in order to increase SCI values based on the density of dead wood. Unfortunately, exactly how these two spatial layers are combined is somewhat arbitrary since it is difficult to quantify the impact of the presence of dead wood relative to the SCI. An example of how the two indicators could be combined is shown in Figure 25, where both the original SCI and the map of dead wood density were simply linearly rescaled from 0 to 100 and added together. In this case values of 0 represent extremely low complexity in the original SCI as well as a complete lack of detected dead wood, while values of 100 represented extremely high complexity and the scaled maximum amount of detected dead wood.

This combined product was not validated in detail, a result of the fact that it was somewhat subjectively created in terms of how the two indicators were combined. 
Instead it is presented as an example of how the two indicators could be used to improve mapping and monitoring efforts. Figure 26 shows and excellent example of the benefits of combining the indicators with an improved representation of one of the independent regions that was used to validate the original SCI. This region had been found to be partially correctly classified as having low structural complexity, however, the results in this case showed that modifying the SCI using the map of dead wood density actually produced a more realistic spatial distribution of predicted complexity within this validation region. 


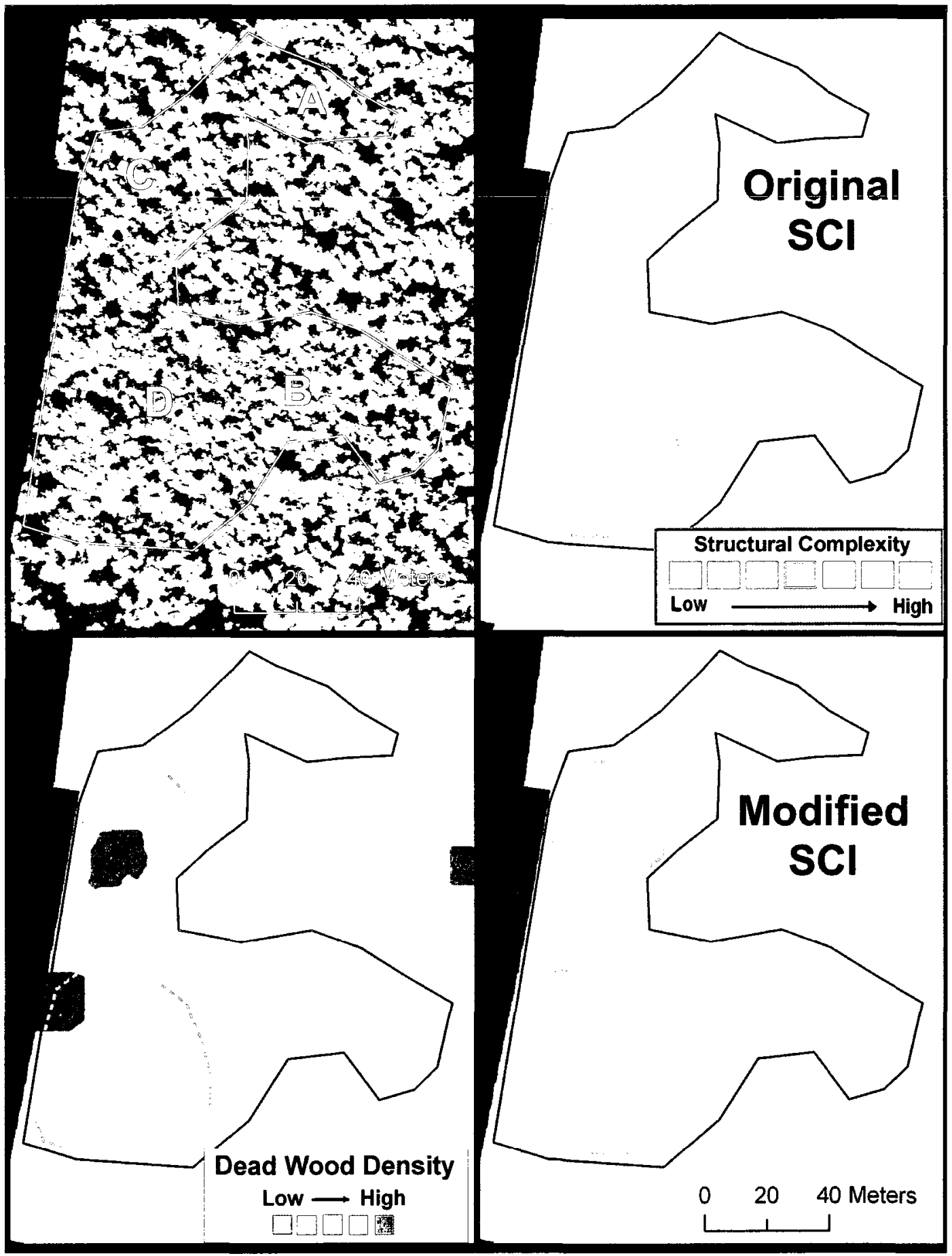

Figure 26. An example area showing results of a combination of indicators. Areas labeled as A and B were correctly classified as low complexity, however those labeled as $\mathrm{C}$ and $\mathrm{D}$ were found to be misclassified and during field validation were found to contain many snags. In this example, the modification of the original SCI using the dead wood density map produced a more realistic representation of the structural complexity compared to the SCI by itself. 


\subsection{Discussion}

The following section presents some discussion of the results, and their applicability, certain limitations within the analyses, as well as further research questions that arose.

\subsection{Development of an Image-Based Structural Complexity Index using RDA}

While the first RDA axis only provided an explanation for $35 \%$ of the variance in the field data, through an analysis of correlations with the individual field variables it was shown to represent a general gradient of structural complexity and was never expected to be used to predict actual values of the individual field variables.

There were several potential sources for the unexplained variance including measurement error, as well as the inherent randomness associated with a natural system. The topographic variables represented the general light regime and macrosite characteristiscs, however microsite characteristics that are known to be related to damage susceptibility and regeneration were not included. Soil type, depth and rockiness (e.g. Diaci et al., 2005; Gillespie et al., 2006; Schmoeckel and Kottmeier, 2008), which are known to affect the structure of forests, were not included in the analysis since these variables were not thought to manifest themselves directly or indirectly in the imagery. Additionally, the varying resolution of the imagery with changing topography might have introduced some error in the extraction of image variables.

The results were considered successful compared to other multivariate forest structural modelling research that used remotely-sensed variables for predictors. While 
difficult to compare directly to the work by Coops and Catling (1997a, 1997b) and Coops et al. (1998), the inclusion of the actual raw field data that was measured in the forest avoided the subjectivity and problems of repeatability that are possible when carrying out qualitative scoring to measure structural complexity as was done in their research.

Cosmopoulos and King's (2004) forest structure index (FSI), which was produced through a pseudo-RDA analysis, accounted for approximately $26 \%$ of the original field data. Their PCA of the field data retained $81.3 \%$ of the original variance, while the CCA accounted for $53 \%$ of the remaining variance, and finally the regression model explained $61 \%$ of that variance, resulting in an explanation of only $26 \%$ of the original variance of the raw data. The complicated nature of such an analysis in terms of the interpretation required at each step supports the use of RDA for this type of multivariate modelling. In any case, similar to the results of this research, the FSI presented by Cosmopoulos and King (2004) used mostly spatial information (4 out of the 5 predictor variables) for explaining forest structural variability as it manifested itself in the imagery. In fact, the variation of GLCM texture was the first variable entered into the model providing $\sim 62 \%$ of the model's explanation, which was similar to the SCI presented in this thesis where the variation of GLCM Homogeneity (from within-crowns) provided $\sim 73 \%$ of the model's explanation. This was arrived out simply by entering the five predictor variables into a multiple regression using the $\mathrm{SCI}$ as the dependent variable since the $\mathrm{SCI}$ was already known to be a linear combination of the five variables.

The importance of spatial information was noted by Wunderle et al. (2007), who included two GLCM textures in their model along with a measure of spectral reflectance. 
While their results showed a high correspondence between mapped structural complexity through jackknifing and their original modeled structural complexity $\left(\mathrm{R}^{2}=0.79\right)$, they lacked independent field validation. In contrast, a field validation was carried out in this thesis, on top of the bootstrapping that was done, in order to provide a more complete validation of such modelling. This approach is always desirable whenever carrying out any sort of predictive modelling using remote sensing.

Compared to the other two existing studies that used RDA for modelling aspects of structural complexity, the current research is the only one that included any spatial information. Sampson et al. (2001) carried out a basic analysis using only a few spectral indices derived from airborne imagery and found weak relations that were reliant on a single variable (canopy openness), which was only a result of the inclusion of clearcut plots. The work by de la Cueva (2008), which modelled forest structure using Landsat spectral information and topographic variables, presented a maximum of only $24 \%$ explanation when combining both the first and second RDA axes. The lack of spatial information, which was not available at the plot scale using Landsat imagery, was a major limitation of their research. As shown in the current research, forest structure can be better explained using image spatial information, including within-crown and withinshadow information, rather than spectral information alone.

Interestingly, in terms of variables included in the final model, all variables, other than IM5 (elevation), represented within-crown and within-shadow information, as opposed to information extracted across the entire plot. Originally (see Section 4.3.3.1) it was hypothesized that areas of the forest with higher complexity would have lower 
reflectance compared to areas with lower complexity as a result of the presence of canopy gaps, representing heterogeneity within the plot. Variables representing full plot spectral information were only included in 5 out of the 500 models created within the bootstrapping procedure and furthermore comparing the average reflectance of the three spectral variables to the SCI showed no relations (NIR: $r=-0.19(p=0.19)$, Red: $r=-$ $0.15(\mathrm{p}=0.31)$, and Green: $\mathrm{r}=-0.11(\mathrm{p}=0.50))$. It had also been hypothesized that areas of higher complexity would be associated with greater spatial variability in image brightness due to the greater presence of gaps / shadows in the overstorey. Full plot GLCM textures (i.e. spatial information) were only included in 54 of the 500 models, with within-crown and within-shadow spatial information included in its place for reasons previously discussed in Section 4.3.3.2. Interestingly first order textural information (standard deviation of image brightness) measured across the entire plot was found to be highly correlated (NIR: $r=0.72$, Red: $r=0.77$, and Green: $r=0.76$ (all $p<$ $0.001)$ ) with the SCI, however, these three variables were only included in 6 of the 500 models. These variables were found to be highly correlated with within-crown textural measures, which were included in $70 \%$ of the models since they provided more explanation compared to the first order full plot spatial measures.

Lastly, in temrs of the variables included in he models, the fact that topographic information was included was not surprising, and actually expected given the know relations between topography and illumination with forest structure as presented in Section 4.3.3.4. 
An important point to make with respect to the modelling presented in this thesis is that RDA was used for more than simply examining relations between sets of variables. Instead, the inherent structure of the RDA was fully exploited in order to develop an empirical model that was used for predictive mapping of the SCI.

Another important advantage of RDA in this research is that multicollinearity was not a major issue. Ordination techniques involve a reduction of the dimensionality of a dataset and therefore inclusion of multiple response (field) variables accounting for some of the same variance does not matter. Within RDA the dimensionality is reduced using a PCA and therefore multiple correlated field variables, for example as shown by clustered structural attributes (blue arrows) in Figure 12, are reduced to a single vector. As well, using a forward stepwise selection for entering predictor variables into the model reduced collinearity issues between predictor variables since variables are entered sequentially into the model based on their ability to explain residual variance in the field variables (Legendre and Legendre, 1998).

\subsubsection{Model Scalability}

The results of testing the scalability of the RDA modelling methods showed that there were significant changes with respect to the types of information present in the imagery at different resolutions. This was evident by the types / sources of information that were included in the models at the different spatial resolutions. Degrading the imagery obviously resulted in a loss of spatial information, and within-crown shadows began to disappear in the $60 \mathrm{~cm}$ imagery, while many of the significant canopy gaps visible in the original $20 \mathrm{~cm}$ imagery were almost completely lost in the $1 \mathrm{~m}$ imagery. 
While the types of information used to build the RDA models changed with scale, the amount of explanation the different models provided with respect to the variance in the field data was not reduced as much as was originally expected. Explained variance decreased by only $3 \%$ and $5 \%$ for the $60 \mathrm{~cm}$ and $1 \mathrm{~m}$ models, respectively, from the original $35 \%$ explanation provided by the $20 \mathrm{~cm}$ model. This was not thought to be a significant decrease and suggested that perhaps lower resolution airborne imagery or possibly high resolution satellite imagery could be used for modelling the overall structural complexity within the forest. However, the error associated with the models increased from $19.9 \%(20 \mathrm{~cm})$ to $24.9 \%(60 \mathrm{~cm})$ and $28.4 \%(1 \mathrm{~m})$, which was an increase of $5 \%$ and $9 \%$, respectively.

These results were promising, however, validation of the new maps using field validation sites showed overall accuracy to be $63.4 \%(60 \mathrm{~cm})$ and $49.7 \%(1 \mathrm{~m})$ compared to $78.5 \%$ accuracy found using the $20 \mathrm{~cm}$ imagery. The large discrepancy between model and map accuracies was unexplainable and further field exploration may be required. Results did seem to indicate that subpixel methods to extract fractional information might be useful with coarser resolution imagery, however, this would require further testing involving imagery acquired at a coarser resolution as opposed to simulated imagery.

Related research by Lévesque and King (2003), who acquired airborne imagery with $25 \mathrm{~cm}, 50 \mathrm{~cm}$ and $1 \mathrm{~m}$ resolutions found the $25 \mathrm{~cm}$ resolution to be best suited for modelling of tree crown structure using similar variables to the current study, however they concluded that of the resolutions evaluated, the $50 \mathrm{~cm}$ models produced the most 
consistent results, providing similar or better models than the others. Without acquiring imagery at multiple resolutions it is difficult to properly test the scalabity of the RDA modelling and mapping method, however, the results do, for the resampled $60 \mathrm{~cm}$ imagery, show strong potential for SCI. This also opens up the potential for using high

resolution satellite imagery, which would provide much greater coverage compared to airborne imagery for the same angle of view.

Further research must be carried out in order to test the use of high resolution satellite imagery, as well as the use of pan-sharpened satellite imagery, which can potentially provide the necessary spatial resolution needed to derive the image variables. A related ongoing research project (V. Torontow and D. King, unpublished data) will be testing the use of pan-sharpened Quickbird imagery $(\sim 60 \mathrm{~cm}$ panchromatic band) with the methods presented in this thesis in order to map low, medium, and high structural complexity in a similar forest outside Gatineau Park. Results from this project will hopefully be conclusive with respect to the potential scalability of these methods.

\subsubsection{Applicability of Results, Research Limitations, and Future Research}

The identification of sites that potentially provide habitat for a variety of species and therefore might have high biodiversity value is important for conservation purposes (Noss, 1999; Smith et al., 2008). At the regional scale, forests could be compared to one another in terms of the amount of valuable habitat that they might provide, and this indicator could possibly be used to prioritize protection of forests. As well, at a finer scale, looking at sites of high value within a forest is important for conservation of habitat within that forest. 
At the same time, locating areas with potentially relatively low biodiversity is also important and could be useful for remediation purposes to possibly increase biodiversity levels. For example, Chambers et al. (2002) stressed the importance of protecting snags in ponderosa pine forests in Arizona in order to provide critical roosting habitat for a variety of bat species. As part of their research they found that the creation of artificial roosts (both wood and resin) throughout the forest, designed to replace snags that were lacking as a result of both natural causes and management practices, successfully enhanced bat habitat. Similarly, Pollard and Moi (2006) went so far as to install snags and CWD piles within a commercial forest in British Columbia, Canada in order to enhance the available habitat in the region. Activities such as this could be better focused if areas with low structural complexity or those lacking dead wood relative to the surrounding forest could be mapped and located using remotely-sensed imagery.

It must be stressed that the methods and results presented in this thesis are by no means intended for provincial or national scale mapping and monitoring programs but rather for local scale forest management. As well, while the reserach was carried out within a temperate hardwood forest, where the selected study area contained only hardwood trees in order to control for structural variation caused by compositional differences, the methods for developing models and maps could be more generally applied in different forest types. A set of field / training plots in a different type of forest could be set up and the methods presented could be followed in order to derive an SCI model. This SCI could then be applied spatially to map the entire study area. Having said this, further research should investigate the similarities and differences between forest types with respect to the predictor variables that are most useful for explaining structural 
complexity, especially since deciduous, mixed, and coniferous forest plots all have different structural compositions.

High resolution imagery is limited in terms of its spatial coverage and while camera systems do exist that provide much greater coverage in a single frame compared to the camera used in this research, view angle issues may become a problem. These types of issues, for the most part, were eliminated from this analysis. In terms of applying the methods for management, high resolution mapping could potentially be acquired over a set of monitoring sites within a very large forested area. These sites could be selected manually on the ground to represent different environments in the forest or in areas susceptible to external stresses. The targeted sites, covered by only a few high resolution images, which would allow for the use of a limited camera view angle, could then be monitored over time using high resolution imagery to capture in detail any changes that may occur at these locations. This approach is preferable to relying on lower resolution imagery that might not provide the same within and inter-object information, which has been shown to be useful for modelling structural complexity.

In terms of applying the results of this research to future study sites, the number of field measurements could be reduced since many of the structural attributes that were incorporated into the SCI were highly correlated. The triplot (Figure 12) and bivariate correlations could be examined in order to reduce the number of attributes needed. Fewer field measurements would allow for a larger sample of field plots to be used for model creation as well as validation. Future research investigating the reduction of field 
variables and the impact on the ability to differentiate between complexity levels is of interest.

Additional remotely-sensed information could potentially be incorporated into this study in an attempt to improve the SCI. Lidar data has been successfully used to model individual structural attributes within predominantly coniferous forests at a variety of scales (e.g. Lefsky et al., 2002; Popescu et al., 2004; Hyde et al., 2005; Lefsky et al., 2005; Hudak et al., 2006; Breidenbach et al., 2008) and therefore may offer the greatest potential for improving models of forest structure. Popescu et al. (2004) investigated the use of lidar data collected under leaf-off conditions over plots of varying compositions in Virginia, U.S.A., including hardwood and pines separately. Regression models for pine plots had $\mathrm{R}^{2}$ values ranging from $0.77-0.90$ for different structural attributes, while the deciduous plots only had $\mathrm{R}^{2}$ values ranging from $0.25-0.51$. These results suggested that, while perhaps not as useful in deciduous forests, lidar does have some potential for providing additional structural information, possibly under leaf-on, but as well under leaf-off conditions, to be used in conjunction with multispectral information. Anderson et al. (2008) tested the use of lidar data and airborne hyperspectral (AVIRIS) imagery with a pixel size of about $16-17 \mathrm{~m}$ for modelling structural attributes measured in a mixed conifer-hardwood forest in north central New Hampshire, U.S.A., including plot basal area, above ground biomass, and stem density (plot size was $0.1 \mathrm{ha}$ ). Their results showed that, for most of the individual structural attributes modeled using variables extracted from both data sources, regression coefficients were highest when using the combined datasets $\left(R^{2}\right.$ values ranged from $0.32-0.55$ for different attributes) rather than each individually. 
Lim et al. (2003) explored the use of lidar to estimate biophysical properties of tolerant hardwood forests composed mainly of sugar maples and yellow birch. They used first and last returns, along with intensity information, collected under leaf-on conditions with approximately 3-5 points $/ \mathrm{m}^{2}$. Their results showed strong relations between laser variables and measured tree heights (maximum $\mathrm{R}^{2}=0.86$ ), mean plot dbh (maximum $\mathrm{R}^{2}$ $=0.46$ ), total basal area (maximum $R^{2}=0.90$ ), percent canopy openness (maximum $R^{2}=$ 0.76 ), LAI (maximum $\mathrm{R}^{2}=0.82$ ), ellipsoidal crown closure (maximum $\mathrm{R}^{2}=0.89$ ), aboveground biomass (maximum $\mathrm{R}^{2}=0.85$ ), as well as stem density (maximum $\mathrm{R}^{2}=$ 0.58). These results show obvious potential for using lidar information when investigating multivariate forest structure, including the derivation of a general index as opposed to individual biophysical properties of a hardwood forest.

While lidar has been used to model individual structural attributes, to date, there do not appear to be any attempts to model and map multivariate structural complexity using multispectral imagery along with lidar information. Perhaps the most similar analysis to that presented in this thesis is that of Lefsky et al. (2005). Their work, carried out in coniferous forests in Washington and Oregon, U.S.A., used a Canonical Correlation Analysis (CCA) in order to investigate relations between lidar variables $(n=$ 29, including canopy height indices, profile indices, transmittance indices, and canopy volume indices) with a set of field measured structural attributes $(n=18$, including LAI, basal area, dbh measures, height measures, and canopy cover). The results showed seven statistically significant pairs of canonical variables, although only the first two really provided a significant amount of explained variance (61\% and $15 \%$, respectively). Their analysis presented simple indices (the canonical variables) theoretically capable of 
quickly assessing information within a large dataset. Based on the findings of others, further research combining leaf-on and / or possibly leaf-off lidar data with high resolution airborne imagery in order to attempt to improve predictive models of structural complexity would certainly be worthwhile and interesting. In the future, very high resolution satellite imagery, providing both multispectral and laser information will hopefully be available as well, providing much larger spatial coverage with sufficiently narrow view angles.

Finally, from a context perspective, following a review of the literature, the assumption was made that higher structural complexity in the forest should provide a greater number and wider variety of habitats to be exploited by species in this forest. While this assumption is easy to accept and could be assessed qualitatively, this thesis did not test the direct relation between structural complexity and biodiversity. While an assessment of the biodiversity within the fifty field plots would have provided some answers to this question, time and resource constraints, as well as a primary focus on remote sensing methods, did not permit such data to be collected

\subsection{Modelling and Mapping Dead Wood}

\subsubsection{Direct Detection of Dead Wood}

An investigation based on detailed field notes as well as an examination of the imagery in detail allowed most of the errors of detected wood ojbects to be explained. The accuracy for detecting dead wood objects was increased from $82 \%$ to $94 \%$, and 
most importantly the results suggested that some objects detected in the imagery were smaller than originally thought possible.

An examination of the control site errors revealed that the errors, and thus a limitation of the methods, were mainly a result of the camera's view angle. Despite the narrow view angle ( $9.5^{\circ}$ from nadir in all directions), some partially dead crowns were not visible to the sensor. These results demonstrated that no matter how well remote sensing algorithms work in detecting dead wood in imagery, the method should still only be used as a sampling, albeit a spatially continuous sampling, of the actual snags found in the forest. This limitation was due to the fact that many snags without any branches intact, as well as those snapped off below the overstorey, are not regularly detectable. In order to fully determine the proportion of dead wood detected in relation to that actually present, an intense survey of all of the dead trees in the forest would have to be conducted.

\subsubsection{Indirect Modelling of Dead Wood}

Unfortunately the indirect modelling of dead wood did not provide very useful results, with standard errors too high to accurately predict the amount of CWD (Adjusted $\mathrm{R}^{2}=0.30, \mathrm{SE}=62.1 \%$ ) or snags (Adjusted $\mathrm{R}^{2}=0.33, \mathrm{SE}=57.2 \%$ ) across the forest. Regression results were disappointing compared to the study by King et al. (2005), which was carried out in the same forest. It is thought that the closeness of those measurements to the 1998 ice storm was potentially the reason for the successful indirect modelling of CWD using upper canopy spectral and spatial measures. Their plots represented a distinct gradient of damage from none to severe, the latter with large gaps, shadows and broken 
crowns (with associated CWD underneath) manifested as quite different image brightness and texture than in less damaged plots. The plots in this research were not specifically selected based on overstorey damage, and therefore it is possible that the same gradient found by King et al. (2005) did not exist. As well, this research was carried out approximately ten years following the storm, and while most of the CWD produced by the ice storm damage remained (Guo et al., 2006; Beets et al., 2008), the canopy had recovered relatively quickly (King and Bemrose, 2005). King et al.'s (2005) model included mean plot NIR brightness, the standard deviation of the NIR band, and mean plot red brightness as predictors of the number of downed branches, but in the current research they did not contribute significantly to the models produced.

Relations between the number of snags and image predictor variables were also poorer than expected. A contributing factor to this was clearly the fact that image variables were extracted over a $400 \mathrm{~m}^{2}$ area, which corresponded to the plot-based structural complexity research. This scale was used since indirect modelling was tested in comparison to direct detection methods, which were carried out at a much finer scale. Image information extracted across the entire plot contained information from the vegetation and gaps that surrounded the snags, however snags made up a very small percentage of each plot in the imagery $(\overline{\mathrm{x}}=1.4 \%$ and maximum was only $9.7 \%)$. As a result, the information represented by the predictor variables potentially contained a large portion of noise, as opposed to information directly or indirectly associated with the snags. A smaller area could have been used for this research, possibly an area that corresponded with the size of crowns in the study area, however this would have resulted 
in an analysis that would have been almost identical to the direct detection methods that relied on local spectral and spatial information in the imagery.

In any case, these modelling results led to the conclusion that, despite significant relationships of canopy spectral and spatial variables with CWD and number of snags, indirect modelling of dead wood in temperate hardwood forests using high resolution imagery does not have great potential for accurate spatial mapping. While Pesonen et al. (2008) did not arrive at useful results using airborne laser data in mixed wood forests in Finland, Bater et al. (2007) did present good results predicting snags in coniferous forest stands in British Columbia, Canada, with models based on lidar derived predictor variables with an $\mathrm{R}^{2}$ as high as 0.75 . Potentially lidar data used in conjunction with spectral and spatial variables extracted from airborne imagery might improve indirect modelling results in a temperate hardwood forest.

\subsubsection{Applicability of Results, Research Limitations, and Future Research}

The direct mapping method presented was able to produce a continuous and extensive map of the approximate distribution of dead wood across the study area. Compared to the time required to carry out field-based sampling, processing imagery and producing a map from remotely-sensed imagery is much faster. The acquisition of high resolution airborne imagery, such as was used for this analysis, can still be time consuming in terms of processing the individual images and creating a mosaic, however, commercially available automated acquisition and processing of digital aerial images would obviously greatly reduce this effort. While high resolution satellites do exist that could provide much greater coverage compared to an airborne imagery, the spatial 
resolution of the multispectral bands of these sensors is not considered to be appropriate for pixel level detection of dead wood in a temperate hardwood forest with the accuracy found in this study. Too much of the multispectral signal would be mixed and only the largest snags would be detectable. However, spectral unmixing may improve on this and warrants further research.

One thing that must be kept in mind was that the dead trees detected in the study area, for the most part, were completely defoliated and quite small (average area $=4.41$ $\mathrm{m}^{2}, \sim 1.18 \mathrm{~m}$ diameter using a circular approximation). The size of the dead wood objects that were detected matched reasonably well with the measured crown diameters of 1056 live trees in the fifty field plots which had an average crown diameter of $3.94 \mathrm{~m}^{2}$ (S.D. = $1.28 \mathrm{~m}^{2}$, minimum $=1.50 \mathrm{~m}^{2}$, maximum $=14.30 \mathrm{~m}^{2}$ (note that testing showed repeated crown diameter measurements to have a standard deviation of $0.70 \mathrm{~m}$ )). Similar research detecting dead trees in hardwood forests included that of Kelly et al. (2004), Guo et al. (2007), and Meentemeyer et al. (2008) who used imagery with resolutions of $<1 \mathrm{~m}, \sim 1$ $\mathrm{m}$, and $33 \mathrm{~cm}$, respectively, all carried out in Californian forests for detection of oak mortality caused by a pathogen. Statistics were not presented in their research with regard to the crown size of the mapped dead trees, but the relative success of these coarser resolution studies may have been due to larger trees than those of this study or detection of foliage colour change typical of sudden oak death (Goheen, 2003). However, to detect smaller dead wood such as that shown in Figure 9, higher resolution imagery is required.

These methods could be developed to be used across any forest of interest, since the detection relied on the general spectral differences between exposed dead branches 
and the surrounding live vegetation, which to some degree exist in any type of forest. Some study areas or types of forests may have large canopy gaps within which the bare ground is visible, and therefore within such areas the user might need to differentiate between bare soil or rocks and dead wood when producing a map of detected wood objects (similarly to Kelly et al., 2004 and Guo et al., 2007).

While it could be argued that the detection methods are not repeatable, they are not meant to be rigid in terms of the parameters of the algorithms, but rather general processing methods that could be used in order to produce a map in a new area of interest. Certain parameters such as the Colour : Shape ratio used for the object-based segmentation might be mainteainted since it was logically based, however, this type of anlaysis will always require a certain level of visual interpretation by the user and as a result will always be a semi-automated process rather than a fully automated one. 


\subsection{RESEARCH SUMMARY ANd CONCLUSIONS}

The research presented in this thesis included methods and results focused on the development of spatially extensive geomatics-based indicators of forest structure. Two distinct, but related, indicators derived from high resolution airborne imagery and topographic information were developed: (1) modelling and mapping forest structural complexity, and (2) the detection and mapping of the spatial distribution of dead wood.

This research used Redundancy Analysis to model forest structural complexity using a suite of image and topographic variables. This was a rarely used approach for developing a structural complexity index, in terms of handling the multivariate aspects of field-based structural complexity and the multivariate data derived from remotely-sensed imagery. Using multiple variables extracted from high resolution airborne imagery, this research has presented new evidence that high resolution image spectral, spatial, and object information combined with topographic information are useful as complementary inputs to multivariate modelling of forest structural complexity. The resultant structural complexity index (SCI) was constructed using five predictor variables representing spectral and spatial properties of crowns and shadows within the forest along with topographic information. It represented the general complexity gradient found in the field data on the first RDA axis and explained $35 \%$ of the total original variance of the field variables. Bootstrapping revealed the model to have a RMSE of $19.9 \%$ of the range of SCI values. A comparison between a qualitative field-based complexity classification (high, medium, and low complexity) and the SCI results provided a similar, although unrelated, result with $18 \%$ of the plots showing confusion between classes. These results 
suggested that the modelling procedures could potentially accurately identify areas of the forest with very high or very low structural complexity.

As an original contribution to the field, and an application of RDA yet to be explored in the literature, the RDA model was directly applied spatially across the entire study area to produce a map of predicted structural complexity. The map was validated using independent field sites and was found to be approximately $80 \%$ accurate. As well, multiscale analysis suggested that while further testing is required, the methods could potentially be scaled up to be used with pan-sharpened high resolution satellite imagery, a change that would allow for much greater spatial coverage and avoid the associated processing needed when working with aerial photography.

This thesis also presented new methods for semi-automated detection and mapping of dead wood within a hardwood forest using high resolution airborne imagery. Colour-infrared airborne imagery was used along with various image processing methods in order to detect and delineate wood objects within the imagery. Validation showed detected dead wood objects to be accurately mapped (94\%), while validation sites mapped as non dead wood were $90 \%$ accurate. While the methods are limited by sensor view angles and the 3-dimensional structure of a forest canopy, they could be used to produce a spatially continuous sampling and map of relative dead wood spatial distribution. As well, the results demonstrate the ability to detect a wide range of sizes of dead wood objects in the imagery. Perhaps one of the most important points from this research is that the methods are repeatable and provide the ability to monitor changes over time in terms of the presence and patterns of dead wood across a forest. Tests of 
indirectly modelling dead wood in the forest using image variables were disappointing. The results led to the conclusion that potentially additional information, perhaps from lidar data acquired in leaf-off conditions, might be used in conjunction with the multispectral information for indirectly modelling downed and standing dead wood.

In conclusion, the results presented in this thesis were deemed to be successful both relative to related research in the existing literature and within the biodiversity conservation context of the research design. The methods presented could be used to derive a map of the spatial distribution of dead wood as well as a map of structural complexity variations across a forest. These maps, used together or separately, could be helpful for forest management through the enhancement of forest inventories and also provide valuable support for research involving carbon budget modelling. More so, within the context of this thesis, they are useful for individual species habitat modelling and mapping, as well as more general biodiversity research in terms of their capacity to help in the assessment, monitoring, and management of forest habitat and biodiversity levels. 


\subsection{REFERENCES}

Akaike, H. 1974. A new look at the statistical model identification. IEEE Transactions on Automatic Control 19(6): 716-723.

Anderson, J.E., L.C. Plourde, M.E. Martin, B.H. Braswell, M-L. Smith, R. O. Dubayah, M.A. Hofton and J.B. Blair. 2008. Integrating waveform Lidar with hyperspectral imagery for inventory of a northern temperate forest. Remote Sensing of Environment 112(4): 1856-1870.

Arsenault, A. 2002. Managing coarse woody debris in British Columbia's forests: A cultural shift for professional foresters. In Proceedings of the Symposium on the Ecology and Management of Dead wood in Western Forests. 1999 November 2-4; Reno, Nevada. Gen. Tech. Rep. PSW-GTR-181. Albany, California: U.S. Department of Agriculture, Forest Service, Pacific Southwest Research Station.

Atauri, J.A. and J.V. de Lucio. 2001. The role of landscape structure in species richness distribution of birds, amphibians, reptiltes and lepidopterans in Mediterranean landscapes. Landscape Ecology 16(2): 147-159.

August, P.V. 1983. The role of habitat complexity and heterogeneity in structuring tropical mammal communities. Ecology 64(6): 1495-1507.

Babyak, M.A. 2004. What you see may not be what you get: A brief, nontechnical introduction to overfitting in regression-type models. Psychosomatic Medicien 66(3): 411-421.

Barker, M.G. and M.A. Pinard. 2001. Forest canopy research: Sampling problems and some solutions. Plant Ecology 153(1-2): 23-38.

Barrios, E. 2007. Soil biota, ecosystem service and land productivity. Ecological Economics 64(2): 269-285.

Bastin, L. 1997. Comparison of fuzzy c-means classification, linear mixture modelling and MLC probabilities as tools for unmixing coarse pixels. International Journal of Remote Sensing 18(17): 3629-3648.

Bater, C.W., N.C. Coops, S.E. Gergel and N.R. Goodwin. 2007. Towards the estimation of tree structural class in northwest coastal forests using Lidar remote sensing. In P. Rönnholm, H. Hyyppä,and J. Hyyppä (eds). Proceedings of ISPRS Workshop on Laser Scanning 2007 and SiliviLaser 2007, September 12-14, 2007, Finalnd. International Archives of Photogrammetry, Remote Sensing and Spatial Information Sciences XXXVI (Part 3/W52), 38-43.

Baydack, R.K. and H. Campa III. 1999. Conserving biological diversity - Principles and perspectives: Setting the context. In Practical Approaches to the Conservation of Biological Diversity. Edited by R. Baydack, H. Campa, and J. Haufler. Island Press, Washington, D.C. 313pp.

Bazzaz, F.A. 1975. Plant species diversity in old-field successional systems in southern Illinois. Ecology 56(2): 485-488. 
Beazley, K. and N. Cardinal. 2004. A systematic approach for selecting focal species for conservation in the forests of Nova Scotia and Maine. Environmental Conservation 31(2): 91-101.

Bechtel, R., A. Sanchez-Azofeifa, B. Rivard, G. Hamilton, J. Martin, and E. Dzus. 2004. Associations between Woodland Caribou telemetry data and Landsat TM spectral reflectance. International Journal of Remote Sensing 25(21): 4813-4828.

Beets, P.N., I.A. Hood, M.O. Kimberley, G.R. Oliver, S.H. Pearceand J.F. Gardner. 2008. Coarse woody debris decay rates for seven indigenous tree species in the central North Island of New Zealand. Forest Ecology and Management 246(4): 548-557.

Bemrose, R.K. 2006. Impacts of radiometric corrections on empirical modelling of biophysical variables with airborne multispectral digitial camera imagery. MSc Thesis, Department of Geography and Environmental Studies, Carleton University, Ottawa, Ontario. 150pp.

Benz, U.C., P. Hofmann, G. Willhauck, I. Lingenfelder and M. Heynen. 2004. Multiresolution object-oriented fuzzy analysis of remote sensing data for GISready information. ISPRS Journal of Photogrammetry \& Remote Sensing 58(3-4): 239-258.

Biodiversity Working Group. 1994. Canadian Biodiversity Strategy, Canada's Response to the Convention on Biological Diversity. http://www.emanrese.ca/eman/reports/publications/rt_biostrat/intro.html

Blackard, J.A., M.V. Finco, E.H. Helmer, G.R. Holden, M.L. Hoppus, D.M. Jacobs, A.J. Lister, G.G. Moisen, M.D. Nelson, R. Riemann, B. Ruefenacht, D. Salajanu, D.L Weyermann, K.C. Winterberger, T.J. Brandeis, R.L. Czaplewski, R.E. McRoberts, P.L. Patterson and R.P. Tymcio. 2008. Mapping U.S. forest biomass using nationwide forest inventory data and moderate resolution information. Remote Sensing of Environment 112(4):1658-1677.

Bragg, D.C., M.G. Shelton and B. Zeide. 2003. Impacts and management implications of ice storms on forests in the southern United States. Forest Ecology and Management 186(1-3): 99-123.

Breidenbach, J., B. Koch, G. Kandler, and A. Kleusberg. 2008. Quantifying the influence of slope, aspect, crown shape and stem density on the estimation of tree height at plot level using Lidar and InSAR data. International Journal of Remote Sensing 29(5): 1511-1536.

Brown, D.G. 2001. A spectral unmixing approach to leaf area index (LAI) estimation at the alpine treeline ecotone. In GIS and Remote Sensing Applications in Biogeography and Ecology. Edited by A.C. Millington, S.J. Walsh, and P.E. Osborne. Kluwer Academic Publishers, Boston.

Bull, E.L. 2002. The Value of Coarse Woody Debris to Vertebrates in the Pacific Northwest. In Proceedings of the Symposium on the Ecology and Management of Dead wood in Western Forests. 1999 November 2-4; Reno, Nevada. Gen. Tech. Rep. PSW-GTR-181. Albany, California: U.S. Department of Agriculture, Forest Service, Pacific Southwest Research Station. 
Buntgen, U., J. Esper, D.C. Frank, K. Nicolussi, and M. Schmidhalter. 2005. A 1052-year tree-ring proxy for Alpine summer temperatures. Climate Dynamics 25(2-3): 141153.

Bursing, R.T. 2005. Tree mortality, canopy turnover, and woody detritus in old cover forests of the southern Appalachians. Ecology 86(1): 73-84.

Butler, R. P. and R. Schlaepfer. 2004. Spruce snag quantification by coupling colour infrared aerial photos and a GIS. Forest Ecology and Management 195(3): 325339.

Butson, C. and D.J. King. 1999. Semivariance analysis of forest structure and remote sensing data to determine an optimal sample plot size. Proceedings 4th International Airborne Remote Sensing Conference and Exhibition (Env. Res. Inst. of Michigan) $/ 21^{\text {st }}$ Canadian Symposium on Remote Sensing (Can. Rem. Sens. Soc.), Ottawa, Ontario, June 21-24. Vol. II, pp. 155-162.

Butson, C.R. and D.J. King. 2006. Lacunarity analysis to determine optimum extents for sample-based spatial information extraction from high-resolution forest imagery. International Journal of Remote Sensing 27(1-2): 105-120.

Cairns, J. 1979. Biological monitoring - concept and scope. In Environmental Biomonitoring, Assessment, Prediction, and Management. Edited by J. Cairns, G.P. Patil, and W.E. Waters. Statistical Ecology Series Vol. 11. International Cooperative Publishing House, Maryland, USA.

Callicott, J.B. 1995. Intrinsic Value in Nature: A Metaethical Analysis. The Electronic Journal of Analytic Philosophy 3.

Canadian Forest Inventory Committee (CFIC). 2004. Canada's National Forest Inventory - Ground sampling guidelines version 4.1, February 13th, 2004. 97pp. https://nfi.nfis.org/documentation/ground_plot/Gp_guidelines_v4.1.pdf.

Canham, C.D. 1988. An index for understorey light levels in and around canopy gaps. Ecology 69(5): 1634-1638.

Carey, A.B. 2003. Biocomplexity and restoration of biodiversity in temperate coniferous forest: Inducing spatial heterogeneity with variable-density thinning. Forestry 76(2): 127-136.

Caro, T.M. and G. O'Doherty. 1999. On the use of surrogate species in conservation biology. Conservation Biology 13(4): 805-814.

Catling, P.C. and R.J. Burt. 1995. Studies of the ground-dwelling mammals of eucalypt forest in south-eastern New South Wales: the effect of habitat variables on distribution and abundance. Wildlife Research 22(3): 271-288.

Chambers, C.L., V. Alm, M.S. Siders and M.J. Rabe. 2002. Use of artificial roosts by forest-dwelling bats in northern Arizona. Wildlife Society Bulletin 30(4): 10851091.

Chen, J.M., and Cihlar, J. 1996. Retrieving leaf area index of boreal conifer boreal forest using Landsat TM images. Remote Sensing of Environment 55(2):153-162. 
Clark, F.J. and F.C. Evans. 1954. Distance to the nearest neighbour as a measure of spatial relationships in populations. Ecology 35(4): 445-453.

Cohen, W.B., T.K. Maiersperger, S.T. Gower and D.P. Turner. 2003. An improved strategy for regression of biophysical variables and Landsat ETM+ data. Remote Sensing of Environment 84(4): 561-571.

Comor, V., J. Orgeas, P. Ponel, C. Rolando and Y.R. Delettre. 2008. Impact of anthropogenic disturbances on beetle communities of French Mediterranean coastal dunes. Biodiversity and Conservation 17(8): 1837-1852.

Coops, N.C. and P.C. Catling. 1997a. Predicting the complexity of habitat in forests from airborne videography for wildlife management. International Journal of Remote Sensing 18(12): 2677-2682.

Coops, N.C. and P.C. Catling. 1997b. Utilising airborne multispectral videography to predict habitat complexity in eucalypt forests for wildlife management. Wildlife Research 24(6): 691-703.

Coops, N.C. and P.C. Catling. 2000. Estimating forest habitat complexity in relation to time since fire. Austral Ecology 25(4): 344-351.

Coops, N.C., D. Culvenor, R. Preston, and P.C. Catling. 1998. Procedures for predicting habitat and structural attributes in eucalypt forests using high spatial resolution remotely sensed imagery. Australian Forestry 61(4): 244-252.

Cosmopoulos, P. and D.J. King. 2004. Temporal analysis of forest structural condition at an acid mine site using multispectral digital camera imagery. International Journal of Remote Sensing 25(12): 2259-2275.

Cowan, D.R. and G.R.J. Cooper. 2008. Merging radiometric grids using histogram matching. Computers and Geoscience 34(3): 291-298.

Curran, P. 1988. The semivariogram in remote sensing: An introduction. Remote Sensing of Environment 24(3), 493-507.

de la Cueva, A.V. 2008. Structural attributes of three forest types in central Spain and Landsat ETM plus information evaluated with redundancy analysis. International Journal of Remote Sensing 29(19): 5657-5676.

de Lima, R.A.F. and L.C. de Moura. 2008. Gap disturbance regime and composition in the Atlantic Montane Rain Forest: the influence of topography. Plant Ecology 197(2): 239-253.

Debinski, D.M., K. Kindscher, and M.E. Jakubauskas. 1999. A remote sensing and GISbased model of habitats and biodiversity in the Greater Yellowstone Ecosystem. International Journal of Remote Sensing 20(17): 3281-3291.

Decocq G, M. Aubert, F. Dupont, D. Alard, R. Saguez, A. Wattez-Franger, B. De Foucault, A. Delelis-Dusollier and J. Bardat. Plant diversity in a managed temperate deciduous forest: Understorey response to two silvicultural systems. Journal of Applied Ecology 41(6): 1065-1079.

Definiens. 2006. Definiens Professional 5 Reference Book. 
DeGayner, E.J., M.G. Kramer, J.G. Doerr, and M.J. Robertsen. 2005. Windstorm disturbance effects on forest structure and black bear dens in southeast Alaska. Ecological Applications 15(4): 1306-1316.

Diaci, J. R. Pisek and A Boncina. 2005. Regeneration in experimental gaps of subalpine Picea abies forest in the Slovenian Alps. European Journal of Forest Research 124(1): 29-36.

Digital Globe. 2008. http://www.digitalglobe.com/index.php/85/QuickBird.

Drapeau, P., J-F. Giroux, F. Moore, A. Nappi and J-P.L. Savard. 2003. Snag use by foraging black-backed woodpeckers (Picoides articus) in a recently burned eastern boreal forest. Auk 120(2): 505-511.

Drever, M.C., K.E.H. Aitken, A.R. Norris, and K. Martin. 2008. Woodpeckers as reliable indicators of bird richness, forest health and harvest. Biological Conservation 141(3): 624-634.

Ducey, M.J., G.J. Jordan, J.H. Gove and H.T. Valentine. 2002. A practical modification of horizontal line sampling for snag and cavity tree inventory. Canadian Journal of Forest Research 32(7): 1217-1224.

Ehrlich, P.R and E.O. Wilson. 1991. Biodiversity studies: Science and policy. Science 253(5021): 758-762.

Englund, S.R., J.J. O'Brien, and D.B. Clark. 2000. Evaluation of digital and film hemispherical photography and spherical densiometry for measuring forest light environments. Canadian Journal of Forest Research 30(12): 1999-2005.

ESRI. 2008. http://www.esri.com/.

Ewers, R.M. and R.K. Didham. 2007. The effect of fragment shape and species' sensitivity to habitat edges on animal population size. Conservation Biology 21(4): 926-936.

Flanders D, M. Hall-Beyer, J. Pereverzoff. 2003. Preliminary evaluation of eCognition object-based software for cut block delineation and feature extraction. Canadian Journal of Remote Sensing 29(4): 441-452.

Franklin, S.E., M.B. Lavigne, M.J. Deuling, M.A. Wulder and E.R. Hunt Jr. 1997. Estimation of forest Leaf Area Index using remote sensing and GIS data for modelling net primary production. International Journal of Remote Sensing 18(16): 3459-3471.

Franklin, J.F., K. Cromack, W. Denison, A. McKee, C. Maser, J. Sedell, F. Swanson, and G. Juday. 1981. Ecological characteristics of old-growth Douglas-fir forests. USDA Forest Service. General Technical Report PNW-118. Pacific North-west Forest and Range Experiment Station, Portland, Oregon. 48pp.

Franklin, S.E., R.J. Hall, L.M Moskal, A.J. Maudie, and M.B. Lavigne. 2000. Incorporating texture into classification of forest species composition from airborne multispectral images. International Journal of Remote Sensing 21(1): 6179 . 
Frazer, G.W., J.A. Trofymow and K.P. Lentzman. 2000. Canopy openness and leaf area in chronosequences of coastal temperate rainforests. Canadian Journal of Forest Research 30(2): 239-256.

Freemark, K.E. and H.G. Merriam. 1986. Importance of area and habitat heterogeneity to bird assemblages in temperate forest fragments. Biological Conservation 36(2) : 115-141.

Frontier, S. 1976. Etude de la décroissance des valeurs propres dans une analyze en composantes principales: comparison avec le modèle de bâton brisé. Journal of Experimental Marine Biology and Ecology 25(1): 67-75.

Gastellu-Etchegorry, J. P., V. Demarez, V. Pinel, and F. Zagolski. 1996. Modeling radiative transfer in heterogeneous 3-D vegetation canopies. Remote Sensing of Environment 58(2):131-156.

GeoEye. 2008. http://www.geoeye.com/CorpSite/products/Default.aspx.

Geospatial Systems Inc. 2007. http://www.geospatialsystems.com/.

Gillespie, T.W., B.R. Zutta, M.K. Early and S. Saatchi. 2006. Predicting and quantifying the structure of tropical forests in south Florida and the neotropics using spaceborne imagery. Global Ecology and Biogeography 15(3): 225-236.

Glowka, L., F. Burhenne-Guilmin, and H. Synge. 1994. A guide to the convention on biological diversity. IUCN. Gland and Cambridge. 161pp

Goheen, E. M. 2003. Detecting, surveying, and monitoring Phytophthora ramorum in forest ecosystems. Sudden Oak Death Online Symposium. www.apsnet.org/online/SOD

Gougeon, F.A. 1995. A crown-following approach to the automatic delineation of individual tree crowns in high spatial resolution aerial images. Canadian Journal of Remote Sensing 21(3):274-284.

Gove, J.H., M.J. Ducey and H.T. Valentine. 2002. Multistage point relascope and randomized branch sampling for downed coarse woody debris estimation. Forest Ecology and Management 155(1-3): 153-162.

Grey, A.N. and D.L. Azuma. 2005. Repeatability and implementation of a forest vegetation indicator. Ecological Indicators 5(1): 57-71.

Griesemer, S.J., T.K. Fuller and R.M. Degraaf. 1998. Habitat use by porcupines (Erethizon dorsatum) in central Massachusetts: Effects of topography and forest composition. American Midland Naturalist 140(2): 271-279.

Grime, J.P. 1979. Plant Strategies and Vegetation Processes. John Wiley and Sons Ltd, New York, NY. 222pp.

Guisan, A., S.B. Weiss, and A.D. Weiss. 1999. GLM versus CCA spatial modelling of plant species distribution. Plant Ecology 143(1): 107-122.

Guo, L.B., E. Bek and R.M. Gifford. 2006. Woody debris in a 16-year old Pinus radiata plantation in Australia: Mass, carbon and nitrogen stocks, and turnover. Forest Ecology and Management 228(1-3): 145-151. 
Guo, Q., M. Kelly, P. Gong, D. Liu. 2007. An object-based classification approach in mapping tree mortality using high spatial resolution imagery. GIScience \& Remote Sensing 44(1): 24-47.

Haara, A. and S. Nevalainen. 2002. Detection of dead or defoliated spruces using digital aerial data. Forest Ecology and Management 160(1-3): 97-107.

Hall, F.G., Y.E. Shimabukuro, and K.F. Huemmrich. 1995. Remote sensing of forest biophysical structure using mixture decomposition and geometric reflectance models. Ecological Applications 5(4): 993-1013.

Hammond, P.M. 1994. Practical approaches to the estimation of the extent of biodiversity in speciose groups. Philosophical Transactions of the Royal Society of London B 345: 119-136.

Hammond, H.E.J., D.W. Langor and J.R. Spence. 2001. Early colonization of Populus wood by saproxylic beetles (Coleoptera). Canadian Journal of Forest Research 31(7): 1175-1183.

Haralick, R.M., K. Shanmugan, and I. Dinstein. 1973. Textural features for image classification. IEEE Transactions on systems, man, and cybernetics. SMC-3: 610621.

Hartwig, C.L., D.S. Eastman and A.S. Harestad. 2002. Forest age and relative abundance of pileated woodpeckers on southern Vancouver Island. In Proceedings of the Symposium on the Ecology and Management of Dead wood in Western Forests. 1999 November 2-4; Reno, Nevada. Gen. Tech. Rep. PSW-GTR-181. Albany, California: U.S. Department of Agriculture, Forest Service, Pacific Southwest Research Station. 949pp.

He, Z.L.L., X.E. Yang., and P.J. Stofella. 2005. Trace elements in agroecosystems and impacts on the environment. Journal of Trace Elements in Medicine and Biology 19(2-3): 125-140.

Hill, J.K., K.C. Hamer, L.A. Lace, and W.M.T. Banham. 1995. Effects of selective logging on tropical forest butterflies on Buru, Indonesia. Journal of Applied Ecology 32(4): 754-760.

Holben, B.N. and Y.E. Shimabukuro. 1993. Linear mixing model applied to coarse spatial resolution data from multispectral satellite sensors. International Journal of Remote Sensing 14(11): 2231-2240.

Holloway, G.L., J.P. Caspersen, M.C. Vanderwel, and B.J. Naylor. 2007. Cavity tree occurrence in hardwood forests of central Ontario. Forest Ecology and Management 239(1-3): 191-199.

Horn, J.L. 1965. A rationale and test for the number of factors in a factor analysis. Psychometrika 30(2): 179-185.

Hudak, A.T., N.L. Crookston, J.S. Evans, M.K. Falkowski, A.M.S. Smith, P.E. Gessler, and P. Morgan. 2006. Regression modelling and mapping of coniferous forest basal area and tree density from discrete-return Lidar and multispectral data. Canadian Journal of Remote Sensing 32(2): 126-138. 
Hyde, P., R. Dubayah, B. Peterson, J.B. Blair, M. Hofton, C. Hunsaker, R. Knox, and W. Walker. 2005. Mapping forest structure for wildlife habitat analysis using waveform Lidar: Validation of montane ecosystems. Remote Sensing of Environment 96(3-4): 427-437.

Jackson, D.A. 1993. Stopping rules in Principal Components Analysis: A comparison of heuristical and statistical approaches. Ecology 74(8): 2204-2214.

Jakubauskas, M.E. 1996. Canonical correlation analysis of coniferous forest spectral and biotic relations. International Journal of Remote Sensing 17(12): 2323 - 2332.

Jelaska, S.D., O. Antonić, M. Božić, J. Križan and V. Kušan. 2006. Responses of forest herbs to available understorey light measured with hemispherical photographs in silver fir-beech forest in Croatia. Ecological Modelling 194(1-3): 209-218.

Jensen, J.R. 2006. Remote Sensing of the Environment: An Earth Resource Perspective (2nd Edition). Prentice Hall, Upper Saddle Hill, NJ. 592pp.

Johansen, K. and S. Phinn. 2006. Linking riparian vegetation spatial structure in Australian tropical savannas to ecosystem health indicators: Semi-variogram analysis of high spatial resolution satellite imagery. Canadian Journal of Remote Sensing 32(3): 228-243.

Johansen, K., N.C. Coops, S.E. Gergel and Y. Stange. 2007. Application of high spatial resolution satellite imagery for riparian and forest ecosystem classification. Remote Sensing of Environment 110(1): 29-44.

Jolliffe, I.T. 2002. Principal Component Analysis. Second Edition, New York: SpringerVerlag New York, Inc. 487pp.

Jones, D. 2008. FATHOM: A Matlab toolbox for ecological and oceanographic data analysis. http://www.rsmas.miami.edu/personal/djones/matlab/matlab.html.

Jordan, G.J., M.J. Ducey and J.H. Gove. 2004. Comparing line-intersect, fixed-area, and point relascope sampling for dead and downed coarse woody material in a managed northern hardwood forest. Canadian Journal of Forest Research 34(8): 1766-1775.

Karst-Riddoch, T.L., M.F.J. Pisaric, and J.P. Smol. 2005. Diatom responses to $20^{\text {th }}$ century climate-related environmental changes in high-elevation mountain lakes on the northern Canadian Cordillera. Journal of Paleolimnology 33(3): 265-282.

Kaiser, H.F. 1960. The application of electronic computers to factor analysis. Educational and Psychological Measurement 20(1): 141-151.

Kayitakire, F., C. Hamel, and P. Defourny. 2006. Retrieving forest structure variables based on image texture analysis and Ikonos-2 imagery. Remote Sensing of Environment 102(3-4): 390-401.

Kellndorfer, J.M., M.C. Dobson, and L.E. Pierce. 2001. Forest biometrics from ERS and JERS in Michigan. Proceedings IEEE International Geoscience and Remote Sensing Symposium (IGARSS) 2001, Sydney, Australia. pp.780 - 782. 
Kelly, M., D. Shaari, Q. Guo, and D. Liu. 2004. A comparison of standard and hybrid classifier methods for mapping hardwood mortality in areas affected by sudden oak death. Photogrammetric Engineering \& Remote Sensing 70(11): 1229-1239.

Kenning, R.S., M.J. Ducey, J.C. Brissete and J.H. Gove. 2005. Field efficiency and bias of snag inventory methods. Canadian Journal of Forest Research 35(12): 29002910.

Kerr, J.T., T.R.E. Southwood, and J. Cihlar. 2001. Remotely sensed habitat diversity predicts butterfly species richness and community similarity in Canada. Proceedings of the National Academy of Sciences 98(20): 11365-11370.

King, D.J. and R. Bemrose. 2005. Temporal analysis of field measured forest health response (1998-2004) following a severe ice storm. Contract report to the National Capital Commission, Ottawa. 14pp.

King, D.J., I. Olthof, P.K.E. Pellikka, E.D. Seed, and C. Butson. 2005. Modelling and mapping forest ice storm damage using remote sensing and environmental data. Natural Hazards 35(3): 321-342.

Kirby, K.J., C.M. Reid, R.C. Thomas, and F.B. Goldsmith. 1998. Preliminary Estimates of Fallen Dead wood and Standing Dead Trees in Managed and Unmanaged Forests in Britain. The Journal of Applied Ecology 35(1):148-155.

Klein Gebbinck, M.S. 1998. Decomposition of mixed pixels in remote sensing images to improve the area estimation of agricultural field. $\mathrm{PhD}$ Thesis. Faculty of Mathematics and Informatics, University of Nijmegen, Jijmegen, The Netherlands.

Klopfer, P.H. and R.H. MacArthur. 1960. Niche size and faunal diversity. The American Naturalist 94(877): 293-300.

Kneeshaw, D.D. and M. Prevost. 2007. Natural canopy gap disturbances and their role in maintaining mixed-species forests of central Quebec, Canada. Canadian Journal of Forest Research 37(9): 1534-1544.

Laliberte, A.S., A. Rango, K.M. Havstad, J.F. Paris, R.F. Beck, R. McNeely and A.L. Gonzalez. 2004. Object-oriented image analysis for mapping shrub encroachment from 1937 to 2003 in southern New Mexico. Remote Sensing of Environment 93(1-2): 198-210.

Laliberte, A.S., J. Koppa, E.L. Fredrickson, and A. Rango. 2006. Comparison of nearest neighbor and rule-based decision tree classification in an object-oriented environment. IEEE International Geoscience and Remote Sensing Symposium and 27th Canadian Symposium on Remote Sensing, July 31 - Aug. 4, 2006, Denver, CO.

Landres, P.B., J.Verner, and J.W. Thomas. 1988. Ecological uses of vertebrate indicator species: A critique. Conservation Biology 2(4): 316-328.

Langor, D.W. and J.R. Spence. 2006. Arthropods as ecological indicators of sustainability in Canadian forests. Forestry Chronicle 82(3): 344-350. 
Lassau, S.A., D.F. Hochuli, G. Cassis, and C.A.M. Reid. 2005. Effects of habitat complexity on forest beetle diversity: Do functional groups respond consistently. Diversity and Distributions 11(1): 73-82

Lassueur, T., S. Joost and C.F. Randin. 2006. Very high resolution digital elevation models. Do they improve models of plant species distribution? Ecological Modelling 198(1-2): 139-153.

Lawton, J.H., D.E. Bignell, B. Bolton, G.F. Bloemers, P. Eggleton, P.M. Hammond, M. Hodda, R.D. Holt, T.B. Larsen, N.A. Mawdsley, N.E. Stork, D.S. Srivastava and A.D. Watt. 1998. Biodiversity inventories, indicator taxa and effects of habitat modification in tropical forest. Nature 391(6662): 72-76.

Leblanc, S.G. and J.M. Chen. 1998. Applications of the 4-scale radiative-transfer model in the remote sensing of boreal forests. In Proceedings IEEE International Geoscience and Remote Sensing Symposium (IGARSS) 1998. pp.1484-1486.

Leblanc, S.G., J.M. Chen, R. Fernandes, D.W. Deering and A. Conley. 2005. Methodology comparison for canopy structure parameters extraction from digital hemispherical photography in boreal forests. Agricultural and Forest Meteorology 129(3-4): 187-207.

Leblanc, S.G. 2006. Digital hemispherical photography manual v. 1.3b. Canadian Centre for Remote Sensing, Natural Resources Canada, Ottawa. 29 pp.

Leckie, D.G., C. Jays, F.A. Gougeon, R.N. Sturrock, and D. Paradine. 2004. Detection and assessment of trees with Phellinus weirii (laminated root rot) using high resolution multi-spectral imagery. International Journal of Remote Sensing 25(4): 793-818.

Lefsky, M.A., W.B. Cohen, and T.A. Spies. 2001. An evaluation of alternate remote sensing products for forest inventory, monitoring, and mapping of Douglas-fir forests in wester Oregon. Canadian Journal of Remote Sensing 31(1): 78-87.

Lefsky, M.A., W.B. Cohen, G.G. Parker, and D.J. Harding. 2002. Lidar remote sensing for ecosystem studies. BioScience 52(1): 19-30.

Lefsky, M.A., A.T. Hudak, W.B. Cohen and S.A. Acker. 2005. Patterns of covariance between forest stand and canopy structure in the Pacific Northwest. Remote Sensing of Environment 95(4): 517-531.

Legendre, P. and L. Legendre. 1998. Numerical ecology. 2nd English edition. Elsevier Science BV, Amsterdam. $870 \mathrm{pp}$.

LeMay, V. and C. Staudhammer. 2005. Indices of stand structural diversity: Mixing discrete, continous, and spatial variables. Proceedings of the IUFRO Sustainable Forestry in Theory and Practice: Recent Advances in Inventory \& Monitoring, Statistics, Information \& Knowledge Management, and Policy Science Conference, Edinburgh, Scotland, April 5 to 8, 2005. 21pp.

Lepš, J. and P. Šmilauer. 2003. Multivariate analysis of ecological data using CANOCO. Cambridge University Press, U.K., 269 pp. 
Lévesque, J. and D.J. King. 1999. Airborne digital camera image semivariance for evaluation of forest structural damage at an acid mine site. Remote Sensing of Environment 68(2): 112-124.

Lévesque, J. and D.J. King. 2003. Spatial analysis of radiometric fractions from highresolution multispectral imagery for modelling individual tree crown and forest canopy structure and health. Remote Sensing of Environment 84(4): 589-602.

Lhotka, J.M. and E.F. Loewenstein. 2006. Indirect measures for characterizing light along a gradient of mixed-hardwood riparian forest canopy structures. Forest Ecology and Management 226(1-3): 310-318.

Li, X., and A.H. Strahler. 1992. Geometric-optical bi-directional reflectance modeling of discrete-crown vegetation canopy: Effect of crown shape and mutual shadowing. IEEE Transactions on Geoscience and Remote Sensing 30(2): 276-292.

Lim, K., P. Treitz, K. Baldwin, I. Morrison, and J. Green. 2003. Lidar remote sensing of biophysical properties of tolerant northern hardwood forests. Canadian Journal of Remote Sensing 29(5): 658-678.

Lindenmayer, D.B., C.R. Margules, and D.B. Botkin. 2000. Indicators of biodiversity for ecologically sustainable forest management. Conservation Biology 14(4): 941950.

Liu, W., S. Gopal and C. Woodcock. 2004. Uncertainty and confidence in land cover classification using a hybrid classifier approach. Photogrammetric Engineering and Remote Sensing 70(8): 963-971.

Lopoukhine, N. 1974. The forests and associated vegetation of Gatineau Park, Quebec. Forest Management Institute Information Report FMR-X-58, Study FM-72. Ottawa, Ontario.

Luther, J.E., R.A. Fournier, D.E. Piercey, L. Guindon, and R.J. Hall. 2006. Biomass mapping using forest type and structure derived from Landsat TM imagery. International Journal of Applied Earth Observation and Geoinformation 8(3): 173-187.

MacArthur, R.H. and J.W. MacArthur. 1961. On bird species diversity. Ecology 42(3): 594-598.

Maguire, C.C. 2002. Dead wood and the richness of small terrestrial vertebrates in southwestern Oregon. In Proceedings of the Symposium on the Ecology and Management of Dead wood in Western Forests. 1999 November 2-4; Reno, Nevada. Gen. Tech. Rep. PSW-GTR-181. Albany, California: U.S. Department of Agriculture, Forest Service, Pacific Southwest Research Station. 949pp.

Mallinis, G., N. Koutsias, M.Tsakiri-Strati and M. Karteris. 2008. Object-based classification using Quickbird imagery for delineating forest vegetation polygons in a Mediterranean test site. Journal of Photogrammetry and Remote Sensing 63(2): 237-250. 
McComb, W. and D. Lindenmayer. 1999. Dying, dead, and down trees. In Maintaining biodiversity in forest ecosystems. Edited by M.L. Hunter. Cambridge University Press, Cambridge, UK. 714 pp.

McElhinny, C., P. Gibbons, C. Brack, and J. Bauhus. 2005. Forest and woodland stand structural complexity: Its definition and measurement. Forest Ecology and Management 218(1-3): 1-24.

McElhinny, C., P. Gibbons, and C. Brack. 2006. An objective and quantitative methodology for constructing an index of stand structural complexity. Forest Ecology and Management 235(1-3): 54-71.

McGeoch, M.A., M. Schroeder, B. Ekbom and S. Larsson. 2007. Saproxylic beetle diversity in a managed boreal forest: Importance of stand characteristics and forestry conservation measures. Diversity and Distributions 13(4): 418-429.

McKenny, H.C., W.S. Keeton and T.M. Donovan. 2006. Effects of structural complexity enhancement on eastern red-backed salamander (Plethodon cinereus) populations in northern hardwood forests. Forest Ecology and Management 230(1-3): 186196.

Meentemeyer, R.K., N.E. Rank, D.A. Shoemaker, C.B. Oneal, A.C. Wickland, K.M. Frangioso and D.M. Rizzo. 2008. Impact of sudden oak death on tree mortality in the Big Sur ecoregion of California. Biological Invasions 10(8): 1243-1255.

Meir, P., J. Grace, and A.C. Miranda. 2000. Photographic method to measure the vertical distribution of leaf area density in forests. Agricultural and Forest Meteorology 102(2-3): 105-111.

Meyer, P., Itten, K.I., Kellenberger, T., Sandmeier, S., and Sandmeier, R., 1993. Radiometric corrections of topographically induced effects on Landsat TM data in an alpine environment. Journal of Photogrammetry and Remote Sensing 48(4): 17-28.

Mikusinski, G., M. Gromadzki, and P. Chylarecki. 2001. Woodpeckers as indicators of forest bird diversity. Conservation Biology 15(1): 208-217.

Miller, J.B. 1967. A formula for average foliage density. Australian Journal of Botany 15(1): 141-144.

Mills, L.S., M.E. Soule, and D.F. Doak. 1993. The keystone-species concept in ecology and conservation. BioScience 43(4): 219-224.

Moorthy, I., J.R. Miller, J.A.J. Berni, P.J. Zarco-Tejada and L. Qingmou. 2007. Extracting tree crown properties from ground-based scanning laser data. Proceedings IEEE International Geoscience and Remote Sensing Symposium (IGARSS) 2007, Barcelona, Spain. pp.2830-2832.

Nagendra, H. 2001. Using remote sensing to assess biodiversity. International Journal of Remote Sensing 22(12): 2377-2400.

National Capital Commission (NCC). 1998. Digital elevation data for Gatineau Park. NCC, Ottawa, Ontario. 
National Capital Commission (NCC). 2005. Gatineau Park Master Plan. http://www.nccccn.gc.ca/data/2/rec_docs/1768_Master_Plan_e.pdf.

Nelson, R.F., P. Hyde, P. Johnson, B. Emessiene, M.L. Imhoff, R. Campbell, and W. Edwards. 2007. Investigating Radar-Lidar synergy in a North Carolina pine forest. Remote Sensing of Environment 110(1): 98-108.

Neumann, M. and F. Starlinger. 2001. The significance of different indices for stand structure and diversity in forests. Forest Ecology and Management 145(1-2): 91106.

Newsome, A.E. and Catling, P.C. 1979. Habitat preferences of mammals inhabiting heathlands of warm temperate coastal, montane and alpine regions of southeastern Australia. In Ecosystems of the World. Vol. 9A. Heathlands and Related Shrublands of the World. Edited by R.L. Specht. Elsevier. Amsterdam, Netherlands.

Nielsen, M.O. 2004. True orthophoto generation. MSc Thesis, Department of Informatics and Mathematical Modelling, Technical University of Denmark, Lyngby, Denmark. 142pp.

Niemela, J. 2000. Biodiversity monitoring for decision making. Annales Zoologici Fennici 37(4): 307-317.

Noss, R.F. 1990. Indicators for monitoring biodiversity: A hierarchical approach. Conservation Biology 4(4): 355-364.

Noss, R.F. 1999. Assessing and monitoring forest biodiversity: A suggested framework and indicators. Forest Ecology and Management 115(2): 135-146.

Oindo, B.O., R.A. de By, and A.K. Skidmore. 2000. Interannual variability of NDVI and bird species diversity in Kenya. International Journal of Applied Earth Observation and Geoinformation 2(3-4): 172-180.

Oindo, B.O. and A.K. Skidmore. 2002. Interannual variability of NDVI and species richness in Kenya. International Journal of Remote Sensing 23(2): 285-298.

Oki, K., H. Oguma, and M. Sugita. 2002. Sub-pixel classification of alder trees using multitemporal Landsat Thematic Mapper imagery. Photogrammetric Engineering and Remote Sensing 68(1): 77-82.

Olthof, I. and D.J. King. 1997. Evaluation of textural information in airborne CIR digital camera imagery for estimation of forest stand leaf area index. In Proceedings of the First North American Symposium on Small Formation Aerial Photography, Oct. 14-17 1997, Cloquet, MN. pp. 154-164.

Olthof, I. and D.J. King. 2000. Development of a forest health index using multispectral airborne digital camera imagery. Canadian Journal of Remote Sensing 26(3): 166176.

Olthof, I., D.J. King, and R.A. Lautenschlager. 2003. Overstorey and understorey leaf area index as indicators of forest response to ice storm damage. Ecological Indicators 3(1): 49-64. 
Olthof, I., D.J. King, and R.A. Lautenschlager. 2004. Mapping deciduous forest ice storm damage using Landsat and environmental data. Remote Sensing of Environment 89(4): 484-496.

Olthof, I., D. Pouliot, R. Fernandes, and R. Latifovic. 2005. Landsat-7 ETM+ radiometric normalization comparison for northern applications. Remote Sensing of Environment 95(3): 388-398.

Olthof, I. and R. Latifovic. 2007. Short-term response of arctic vegetation NDVI to temperature anomalies. International Journal of Remote Sensing 28(21): 48234840 .

Omari, K., H.P. White, and K. Staenz. 2008. An enhanced description of multiple scattering within the FLAIR model using the photon re-collision probability approach. IEEE Transactions on Geosciences and Remote Sensing. Accepted.

Onianwa, P.C. 2001. Monitoring atmospheric metal pollution: A review of the use of mosses as indicators. Environmental Monitoring and Assessment 71(1): 13-50.

Opdam, P., J. Verboom, and R. Pouwels. 2003. Landscape cohesion: An index for the conservation potential of landscapes for biodiversity. Landscape Ecology 18(2): 113-126.

Ostroumov, S.A. 2005. Some aspects of water filtering activity of filter-feeders. Hydrobiologia 542(1): 275-286.

Palmer, M. 2008. Ordination methods for ecologists. http://ordination.okstate.edu/index.html.

Pasher, J., D.J. King and K. Lindsay. 2007. Modelling and mapping potential hooded warbler (Wilsonia citrina) habitat using remotely sensed imagery. Remote Sensing of Environment 107(3): 471-483.

Patton, D. R. 1975. A diversity index for quantifying habitat edge. Wildlife Society Bulletin 3(4): 171-173.

Payer, D.C. and D.J. Harrison. 2003. Influence of forest structure on habitat use by American marten in an industrial forest. Forest Ecology and Management 179(13): $145-156$.

Pearce, J.L., and L. Venier. 2006. Using ground beetles and spiders as bioindicators of sustainable forest management? Ecological Indicators 6(4): 780-793.

Pearson, D.L. 1994. Selecting indicator taxa for the quantitative assessment of biodiversity. Philosophical Transactions of the Royal Society of London B 345: 75-78.

Peddle, D.R., F.G. Hall, and E.F. LeDrew. 1999. Spectral mixture analysis and geometric-optical reflectance modelling of boreal forest biophysical structure. Remote Sensing of Environment. Vol. 67(3): 288-297. 
Peddle, D.R., S.P. Brunke., and F.G. Hall. 2001. A comparison of spectral mixture analysis and ten vegetation indices for estimating boreal forest biophysical information from airborne data. Canadian Journal of Remote Sensing 27(6): 627635 .

Peddle, D.R., R.L. Johnson, J. Cihlar, and R. Latifovic 2004. Large area forest classification and biophysical parameter estimation using the 5-Scale canopy reflectance model in Multiple-Forward Mode. Remote Sensing of Environment 89(2): 252-263.

Pellikka, P.K.E. 1998. Development of correction chain for multispectral airborne video camera data for natural resource assessment. Fennia 176: 1-110.

Pellikka, P.K.E., E.D. Seed, and D.J. King. 2000. Modelling deciduous forest ice storm damage using CIR aerial imagery and hemispheric photography. Canadian Journal of Remote Sensing 26(5): 394-405.

Peres-Neto, P.R., P. Legendre, S. Dray and D. Borcard. 2006. Variation partitioning of species data matrices: estimation and comparison of fractions. Ecology 87(10): 2614-2625.

Pesch, R. and W. Schroeder. 2006. Mosses as bioindicators for metal accumulation: Statistical aggregation of measurement data to exposure indices. Ecological Indicators 6(1): 137-152.

Pesonen, A., M. Maltamo, K. eerikainen, and P. Packalen. 2008. Airborne laser scanningbased prediction of coarse woody debris volumes in a conservation area. Forest Ecology and Management 255(8-9): 3288:3296.

Phillips, L.B., A.J. Hansen, and C.H. Flather. 2008. Evaluating the species energy relationship with the newest measures of ecosystem energy: NDVI versus MODIS primary production. Remote Sensing of Environment 112(9): 3538-3549.

Pickup, G., V.H. Chewings, and G. Pearce. 1995. Procedures for correcting high resolution airborne video imagery. International Journal of Remote Sensing 16(9): 1647-1662.

Pielou, E.C. 1959. The use of point to plant distances in the study of pattern of plant populations. Journal of Ecology 47(3): 607-613.

Pielou, E. C. 1984. The Interpretation of Ecological Data: A Primer on Classification and Ordination. John Wiley and Sons, New York, NY. 263 pp.

Pisaric, M.F.J., D.J. King, A.J.M. MacIntosh and R. Bemrose. 2008. Impact of the 1998 ice storm on the health and growth of sugar maple (Acer saccharum Marsh) dominated forests in Gatineau Park, Quebec. Journal of the Torrey Botanical Society 135(4): 530-539.

Pollard, B. and A. Moi. 2006. Restoring Structural Diversity to the Kitimat Valley, British Columbia, Canada. http://www.unepwcmc.org/forest/restoration/docs/Kitimat_Valley_Canada.pdf. 
Popescu, S.C., R.H. Wynne, and J.A. Scrivani. 2004. Fusion of small-footprint Lidar and multispectral data to estimate plot-level volume and biomass in deciduous and pine forests in Virginia, USA. Forest Science 50(4): 551-565.

Pouliot, D.A., D.J. King, and D.G. Pitt. 2005. Development and evaluation of an automated tree detection-delineation algorithm for monitoring regenerating coniferous forests. Canadian Journal of Forest Research 35(10): 2332-2345.

Pretzsch, H. 1997. Analysis and modelling of spatial stand structures: Methodological considerations based on mixed beech-larch stands in Lower Saxony. Forest Ecology and Management 97(3): 237-253.

Przybylak, R., J. Majorowicz, G. W'Ojcik, A. Zielski, W. Chor, A. Zyczewsi, K. Marciniak, W. Nowosad, P. Oliski, and K. Syta. 2005. Temperature changes in Poland from the $16^{\text {th }}$ to the $20^{\text {th }}$ centuries. International Journal of Climatology 25(6): 773-791.

Pu, R., P. Gong, and G.S. Biging. 2003. Simple calibration of AVIRIS data and LAI mapping of forest plantation in southern Argentina. International Journal of Remote Sensing 24(23): 4699-4714.

Rao, C.R., 1964. The use and interpretation of principal component analysis in applied research. Sankha 26, 329-358.

Rainio, J. and J. Niemela. 2003. Ground beetles (Coleoptera: Carabidae) as bioindicators. Biodiversity and Conservation 12(3): 487-506.

Ralph, C.J. 1985. Habitat association patterns of forest and steppe birds of Northern Patagonia, Argentina. The Condor 87(4): 471-483.

Red Path Museum - McGill University. 2006. Natural History of Quebec. http:/www.redpath-museum.mcgill.ca/Qbp/Natural\%20History/nat_hist.html\#2.

Riano, D., Chuvieco, E., Salas, J., and Aquado, I., 2003. Assessment of different topographic corrections in Landsat-TM data for mapping vegetation types. IEEE Transactions on Geoscience and Remote Sensing 41(5): 1056-1061.

Richter, R., 2005. Atmospheric / topographic correction for satellite imagery (ATCOR2/3 User Guide Version 6). German Aerospace Centre, Wessling, Germany. pp. 26-27.

Ricketts, T.H., E. Dinerstein, D.M. Olson, and C. Loucks. 1999. Who's where in North America: Patterns of species richness and the utility of indicator taxa for conservation. BioScience 49(5): 369-381.

Rolston, H. 1994. Conserving natural value. Columbia University Press, NY, NY. 259pp.

Ruhland, K. and J.P. Smol. 2005. Diatom shifts as evidence for recent Subarctic warming in a remote tundra lake, NWT, Canada. Palaeogeography, Paleoclimatology, and Paleoecology 226(1-2): 1-16.

Sampson, P.H., P.M. Treitz, and G.H. Mohammed. 2001. Remote sensing of forest condition in tolerant hardwoods: An examination of spatial scale, structure and function. Canadian Journal of Remote Sensing 27(3): 232-246. 
Sanchez, F., A. Serrano, S. Parra, M. Ballesteros and J.E. Cartes. 2008. Habitat characteristics as determinant of the structure and spatial distribution of epibenthic and demersal communities of Le Danois Bank (Cantabrian Sea, N. Spain). Journal of Marine Systems 72(1-4): 64-86

Schmoeckel, J. and C. Kottmeier. 2008. Storm damage in the Black Forest caused by the winter storm "Lothar" - Part 1: Airborne damage assessment. Natural Hazards and Earth System Sciences 8(4): 795-803.

Schuster, B. and M. Diekmann. 2005. Species richness and environmental correlates in deciduous forests of Northwest Germany. Forest Ecology and Management 206(1-3): 197-205.

Seed, E.D. and D.J. King. 1997. Determination of mixed boreal forest stand biophysical structure using large scale airborne digital camera imagery. In Proceedings of GER '97 / 19th Canadian Symposium on Remote Sensing, Natural Resources Canada, May 24-30, Ottawa, Ontario CD-ROM article \#75, 8 pp.

Seed, E.D. and D.J. King. 2003. Shadow brightness and shadow fraction relations with effective LAI: Importance of canopy closure and view angle in mixedwood boreal forest. Canadian Journal of Remote Sensing 29(3): 324-335.

Seto, K.C., E. Fleishman, J.P. Fay, and C.J. Betrus. 2004. Linking spatial patterns of bird and butterfly species richness with Landsat TM derived NDVI. International Journal of Remote Sensing 25(20): 4309-4324.

Settle, J.J. and N.A. Drake. 1993. Linear mixing and the estimation of ground cover proportions. International Journal of Remote Sensing 14(6): 1159-1177.

Shear, H., N. Stadler-Salt, P. Bertram, and P. Horvatin. 2003. The development and implementation of indicators of ecosystem health in the Great Lakes Basin. Environmental Monitoring and Assessment. 88(1-3):119-152.

Skidmore, A.K., B.O. Oindo, and M.Y. Said. 2003. Biodiversity assessment by remote sensing. In Proceedings of the 30th International symposium on remote sensing of the environment: information for risk management and sustainable development, November 10-14, 2003, Honolulu, Hawaii. 4pp.

Smith, W.P. 2007. Ecology of Glacuomys Sabrinus: Habitat, demography and community relations. Journal of Mammalogy 88(4): 862-881.

Smith, R.L. and T.M. Smith. 2001. Ecology and field biology. Benjamin Cummings, NY, NY. 771pp.

Smith, G.F., T. Gittings, M. Wilson, L. French, A. Oxbrough, S. O'Donoghue, J. O'Halloran, D.L. Kelly, F. J.G. Mitchell, T. Kelly, S. Iremonger, A. McKee, and P. Giller. 2008. Identifying practical indicators of biodiversity for stand-level management of plantation forests. Biodiversity and Conservation 17(5):9911015.

Smol, J.P., A.P. Wolfe, H.J.B. Birks et al. 2005. Climate-driven regime shifts in the biological communities of artic lakes. Proceedings of the National Academy of Sciences of the United States of America 102(12): 4397-4402. 
Soenen, S.A., Peddle, D.R., and Coburn, C.A., 2005. SCS+C: A modified sun-canopysensor topographic correction in forested terrain. IEEE Transactions on Geoscience and Remote Sensing 43(9): 2148-2159.

Soudani K, J. Trautmann, and J.M.N. Walter. 2002. Leaf area index and canopy stratification in Scots pine (Pinus sylvestris L.) stands. International Journal of Remote Sensing 23(18): 3605-3618.

Spiering, D.J. and R.L. Knight. 2005. Snag density and use by cavity-nesting birds in managed stands of the Black Hills National Forest. Forest Ecology and Management 214(1-3): 40-52.

Spies, T.A. and J.F. Franklin. 1991. The structure of natural young, mature, and oldgrowth Douglas-Fir forests in Oregon and Washington. In: Aubry, K.B., M.H. Brookes, J.K Agee, R.G. Anthony, and J.F. Franklin (Eds.), Wildlife and vegetation of unmanaged Douglas-Fir forests. USDA Forest Service, Portland, Oregon, pp. 91-109.

St. Onge, B.A. and F. Cavayas. 1997. Automated forest structure mapping from highresolution imagery based on directional semivariogram estimates. Remote Sensing of Environment 61(1): 82-95.

Stahl, G., A. Ringvall, J.H. Gove and M.J. Ducey. 2002. Correction for slope in point and transect relascope sampling of downed coarse woody debris. Forest Science 48(1): 85-92.

Staudhammer, C. and V.M. Lemay. 2001. Introduction and evaluation of possible indices of stand structural diversity. Canadian Journal of Forest Research 31(7): 11051115 .

Steele, B.M. 2000. Combining multiple classifiers: An application using spatial and remotely sensed information for land cover type mapping. Remote Sensing of Environment 74(3): 545-556.

Steinbeiss, S., H. Bessler, C. Engels, V.M. Temperton, N. Buchmann, C. Roscher, Y. Kreutziger, J. Baade, M. Habekost, and G. Gleixner. 2008. Plant diversity positively affects short-term soil carbon storage in experimental grasslands. Global Change Biology 14(12): 2937-2949.

Stephens, S.L. and J.J. Moghaddas. 2005. Fuel treatment effects on snags and coarse woody debris in a Sierra Nevada mixed conifer forest. Forest Ecology and Management 214(1-3): 53-64.

Stevens, V. 1997. The ecological role of coarse woody debris: An overview of the ecological importance of CWD in B.C. forests. Research Branch, British Columbia Ministry of Forestry. Victorial, B.C. Working Paper 30/1997.

Stewart, D. and W.A. Love. 1968. A general canonical correlation index. Psychological Bulletin 70(3): 160-163.

Stribling, H.L. 1990. Bird community response to timber stand improvement and snag retention. Northern Journal of Applied Forestry 7(1): 35-38. 
Sullivan, T.P. and D.S. Sullivan. 2001. Influence of variable retention harvests on forest ecosystems. II. Diversity and population dynamics of small mammals. Journal of Applied Ecology 38(6): 1234-1252.

Tabachnick, B.G. and L.S. Fidell. 1996. Using multivariate statistics. Harper Collins College Publishers, New York. 746pp.

Takahashi, M., Y. Sakai, R. Ootomo and M. Shiozaki. 2000. Establishment of tree seedlings and water-soluble nutrients in coarse woody debris in an old-growth Piceai-Abies forest in Hokkaido, northern Japan. Canadian Journal of Forest Research 30(7): 1148-1155.

Taulman, J.F. 1999. Selection of nest trees by southern flying squirrels (Sciuridae: Glacuomys volans) in Arkansas. Journal of Zoology 248(3): 369-377.

Teillet, P.M., Guindon, B., and Goodenough, D.G., 1982. On the slope-aspect correction of multispectral scanner data. Canadian Journal of Remote Sensing.8(2): 84-106.

ter Braak, C. J. F. 1987. Ordination. In Jongman, R.H., C.J.F. ter Braak and O.F.R. van Tongeren, editors. Data Analysis in Community Ecology. Pudoc, Wageningen, The Netherlands. pp 91-173.

ter Braak, C.J.F. and P. Šmilauer. 2002. CANOCO reference manual and CanoDraw for Windows. user's guide: Software for Canonical Community Ordination (v. 4.5). Microcomputer Power, Ithaca, NY. 500 pp.

Ter-Mikaelian, M.T., S.J. Colombo SJ and J.X. Chen. 2008. Amount of downed woody debris and its prediction using stand characteristics in boreal and mixedwood forests of Ontario, Canada. Canadian Journal of Forest Research 38(8): 21892197.

Tews, J., U. Brose, V. Grimm, K. Tielborger, M.C. Wichmann, M. Schwager, and F. Jeltsch. 2004. Animal species diversity driven by habitat heterogeneity/diversity: The importance of keystone structures. Journal of Biogeography 31(1): 79-92.

Thomas, J.W. 2002. Dead wood: From forester's bane to environmental boon. In Proceedings of the Symposium on the Ecology and Management of Dead wood in Western Forests. 1999 November 2-4; Reno, Nevada. Gen. Tech. Rep. PSWGTR-181. Albany, California: U.S. Department of Agriculture, Forest Service, Pacific Southwest Research Station.

Treitz, P. and P. Howarth. 2000. High spatial resolution remote sensing data for forest ecosystem classification: An examination of spatial scale. Remote Sensing of Environment 72(3): 268-289.

Turner, M.G. and V.H. Dale. 1998. Comparing large infrequent disturbances: what have we learned? Ecosystems 1(6): 493-496.

Ulyshen, M.D., J.L. Hanula, S. Horn, J.C. Kilgo, and C.E. Moorman. 2004. Spatial and temporal patterns of beetles associate with coarse woody debris in managed bottomland hardwood forests. Forest Ecology and Management 199(2-3): 259272. 
United Nations Convention on Biological Diversity (UN-CBD). 1993. Full text access. http://www.cbd.int/doc/legal/cbd-un-en.pdf

Vaillancourt, M., P. Drapeau, S. Gauthier and M. Robert. 2008. Availability of standing trees for large cavity-nesting birds in the eastern boreal forest of Québec, Canada. Forest Ecology and Management 255(7): 2272-2285.

Valentine, H.T., J.H. Gove, and T.G. Gregoire. 2001. Monte Carlo approach to sampling forested tracts with lines or points. Canadian Journal of Forest Research 31(8): $1410-1424$.

Van de Veer, D. and C. Pierce. 1998. The environmental ethics and policy book. Wadsworth Publishing Company, Belmont, CA. 673pp.

Van den Meersschaut, D. and Vandekerkhove, K. 1998. Development of a stand scale forest biodiversity index based on the State Forest Inventory. In Integrated Tools for Natural Resources Inventories in the $21^{\text {st }}$ Century. Eds. M. Hansen and T. Burk, USDA, Boise, Idaho, USA, pp. 340-349.

Van der Sanden, J.J. and D.H. Hoekman. 2005. Review of relationships between greytone co-occurrence, semivariance, and autocorrelation based image texture anlaysis approaches. Canadian Journal of Remote Sensing 31(3): 207-213.

Van Pelt, R. and N.M. Nadkarni. 2004. Development of canopy structure in Pseudotsuga menziesiiforests in the southern Washington Cascades. Forest Science 50(3): 326341.

Van Wagner, C.E. 1964. The line intersect method in forest fuel sampling. Forest Science 14(1): 20-26.

Vega, C. and B. St-Onge. 2008. Height growth reconstruction of a boreal forest canopy over a period of 58 years using a combination of photogrammetric and Lidar models. Remote Sensing of Environment 112(4): 1784-1794.

Venier, L.A. and J.L. Pearce. 2004. Birds as indicators of sustainable forest management. The Forestry Chronicle 80(1): 61-66.

Waring, R.H. 2000. A process model analysis of environmental limitations on the growth of Sitka spruce plantations in Great Britain. Forestry 73(1): 65-79.

Williams, S.E., H. Marsh, and J. Winter. 2002. Spatialscale, species diversity, and habitat structure: Small mammals in Australian tropical rain forest. Ecology 83(4): 13171329.

Wulder, M. 1998. Optical remote-sensing techniques for the assessment of forest inventory and biophysical parameters. Progress In Physical Geography 22(4): 449-476.

Wulder, M.A., E.F. LeDrew, S.E. Franklin, and M.B. Lavigne. 1998. Aerial image texture information in the estimation of northern deciduous and mixedwood forest leaf area index (LAI). Remote Sensing of Environment 64(1): 64-76. 
Wulder, M.A., J.C. White, N.C. Coops and C.R. Butson. 2008. Multi-temporal analysis of high spatial resolution imagery for disturbance monitoring. Remote Sensing of Environment 112(6): 2729-2740.

Wunderle, A.L, S.E. Franklin and X.G. Guo. 2007. Regenerating boreal forest structure estimation using SPOT-5 pan-sharpened imagery. International Journal of Remote Sensing 28(19): 4351-4364.

Xu, S.Y., D.R. Peddle, C.A. Coburn and S. Kienzle. 2008. Sensitivity of a carbon and productivity model to climatic, water, terrain, and biophysical parameters in a Rocky Mountain watershed. Canadian Journal of Remote Sensing 34(3): 245-258.

Yamaura, Y., K. Katoh and T. Takahashi. 2008. Effects of stand, landscape, and spatial variables on bird communities in larch plantations and deciduous forests in central Japan. Canadian Journal of Forest Research 38(5): 1223-1243.

Yan, G., J.F. Mas, B.H.P. Maathuis, Z. Xiangmin, and P.M. Van Dijk. 2006. Comparison of pixel-based and object-oriented image classification approaches - a case study in a coal fire area, Wuda, Inner Mongolia, China. International Jounrla of Remote Sensing 27(18): 4039-4055.

Zawadzki, J., C.J. Cieszewski, M. Zasada, and R.C. Lowe. 2005. Applying geostatiscs for investigations of forest ecosystems using remote sensing imagery. Silva Fennica 39(4): 599-617.

Zenner, E.K. 2000. Do residual trees increase structural complexity in Pacific Northwest coniferous forests? Ecological Applications 10(3): 800-810.

Zenner, E.K. and D.E. Hibbs. 2000. A new method for modeling the heterogeneity of forest structure. Forest Ecology and Management 129(1-3): 75-87.

Zenner, E.K. 2004. Does old-growth condition imply high live-tree structural complexity? Forest Ecology and Management 195(1-2): 243-258.

Zielinski, W.J., R.L. Truex, J.R. Dunk, and T.Gaman. 2006. Using forest inventory data to assess fisher resting habitat suitability in California. Ecological Applications 16(3): 1010-1025. 


\begin{abstract}
APPENDICES
APPENDIX 1: NET BRIGHTNESS CORRECTION PROCEDURE

(1) The following code was written in order to remove brightness variations caused by sun-sensor-surface geometry (bi-directional reflectance variation (BRV) and topographic variations) as well as lens effects (light fall-off / optical vignetting). This code was written in the EASI Scripting language, which runs within the PCI Geomatica environment. The procedure removes brightness variations from a set of raw 3-band images (TIF files) resulting in a set of corrected images. A manually derived mask (TIF) is required for each image, with non-forested areas masked out. The code was written with the help of Nick Walsworth.
\end{abstract}

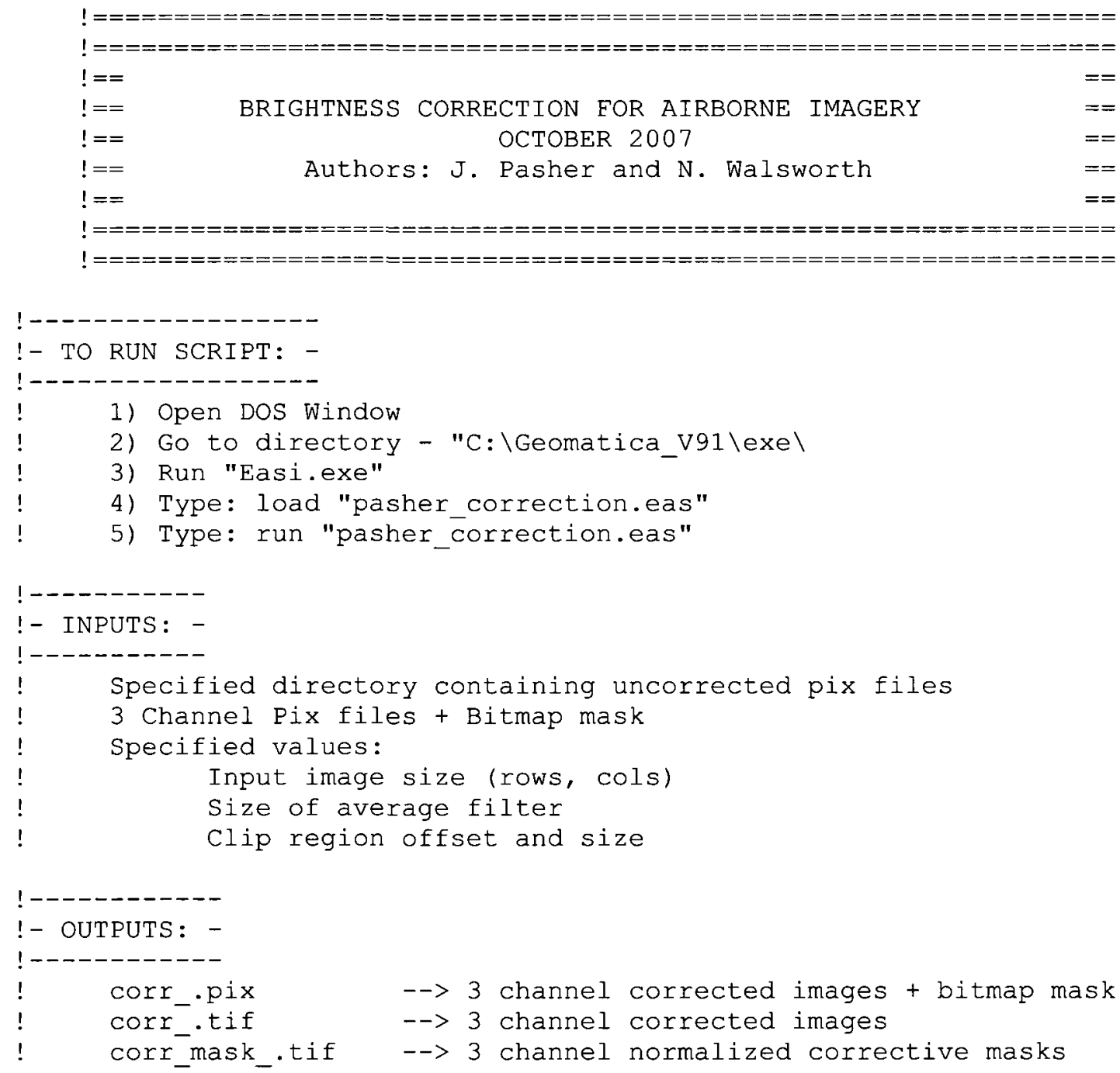


local mstring dirlist

local string infiles, inputfile, copypix, outfile, avgout

local string ext, basename

local int $x, i$, numrows, numcols

local int subwx, subwy, subwxoff, subwyoff

local int filtersize

local $\$ \mathrm{Z}$

local float channelmax[3]

! - ------------

! - SET INPUTS -

!------------

!(1) Directory containing uncorrected subset pix files

in_files = "D: \Student $\backslash$ Test"

!(2) Full size of input image and subset window size and offset

$\begin{array}{ll}\text { subwx } & =420 \\ \text { subwy } & =0 \\ \text { subwxoff } & =1080 \\ \text { subwyoff } & =1080\end{array}$

!(3) Average filter size ( $x$ and $y$ direction)

filtersize $=99$

dirlist $=$ getdirectory (in_files)

let $\$ \mathrm{Z}=" \backslash$

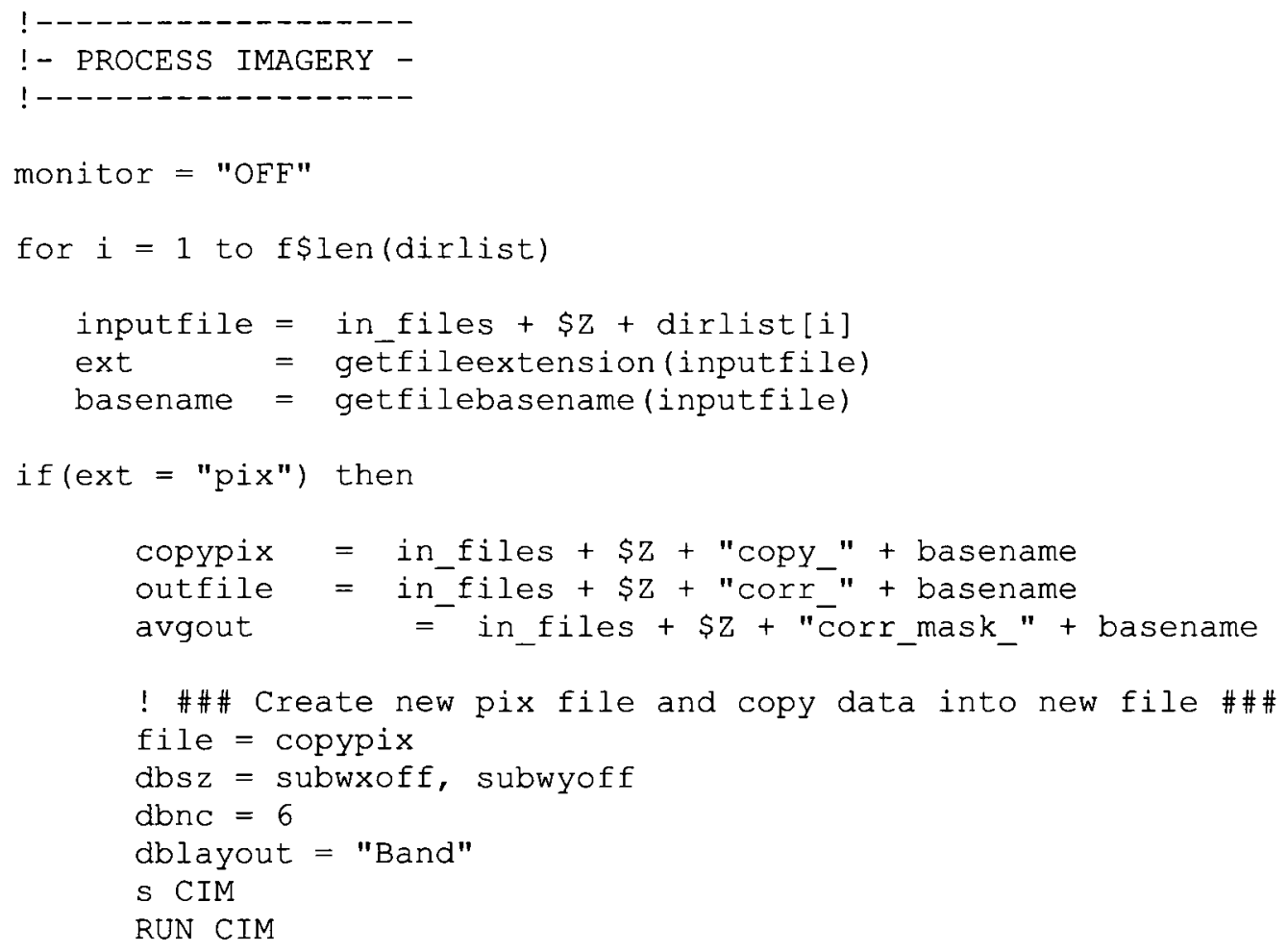




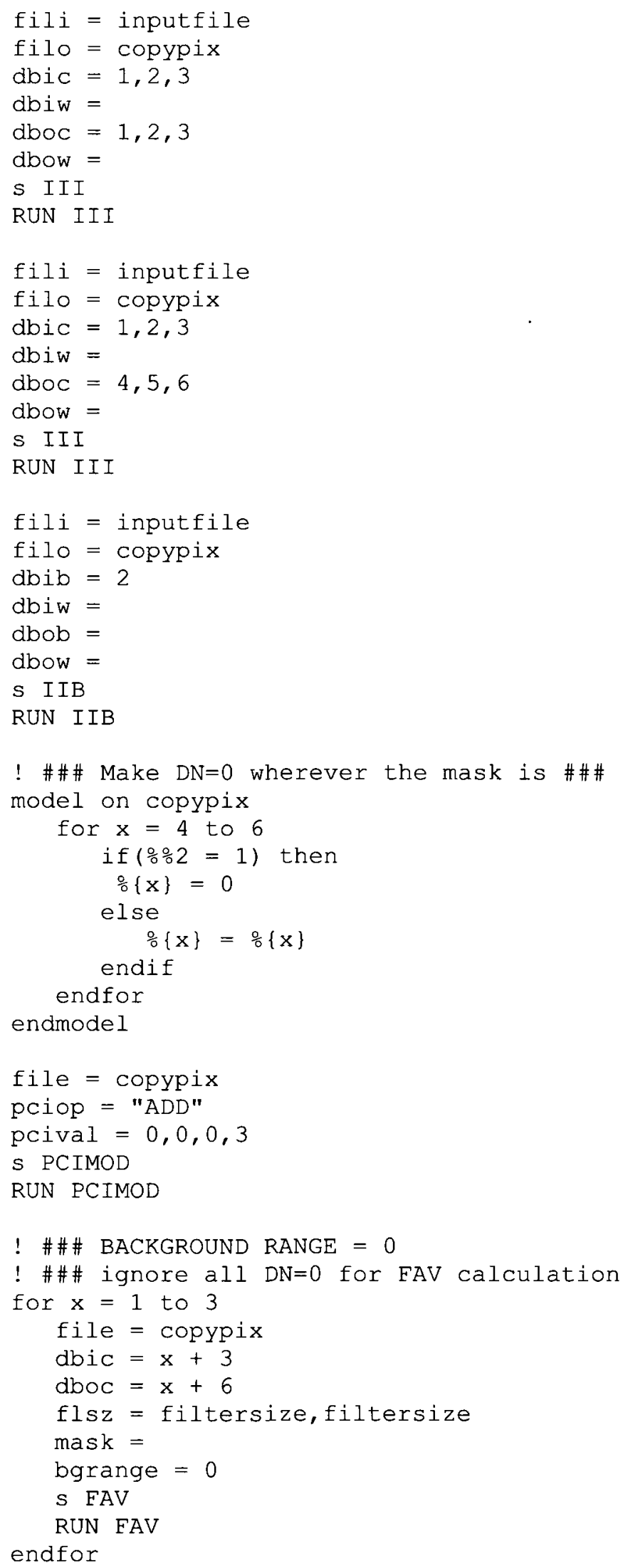


! \#\#\# Get MAX value from each averaged/masked channel \#\#\#

for $\mathrm{x}=1$ to 3

file = copypix

$\mathrm{dbic}=\mathrm{x}+6$

trim $=$

gmod $=$

$\mathrm{cmod}=$ "OFF"

pcmo $=$

nsam $=0$

hisw $=$

mask =

report $=$

$S$ HIS

RUN HIS

channelmax $[\mathrm{x}]=\operatorname{IMSTAT}(12)$

endfor

! \#\# Normalize Brightness mask for correction \#\# model on copypix

$\frac{0}{7}=$ channelmax $[1] / \% 7$

$\div 8=$ channelmax $[2] / \% 8$

$\div 9=$ channelmax[3] / $\div 9$

endmode 1

! \#\#\# Create temporary 3 x 8bit file to hold correction

! \#\#\# Scale corrective mask to 8 bit and output to temp pix file

! \#\#\# Export 3 corrective masks to TIF file and

! \#\#\# delete temp pix file

file = avgout

dbsz = subwxoff, subwyoff

$\mathrm{dbnc}=3$

dblayout = "Band"

S CIM

RUN CIM

for $\mathrm{x}=7$ to 9

fili $=$ copypix

filo $=$ avgout

$\mathrm{dbic}=\mathrm{x}$

$\mathrm{dboc}=\mathrm{x}-6$

$\mathrm{dbiw}=$

dbow $=$

inrange $=$

outrange $=$

sfunct $=$ "LIN"

$s$ scale

RUN SCALE

endfor

! \#\#\# Output correction mask to TIF file \#\#

fili = avgout

filo = avgout + ".tif"

$\mathrm{dbic}=1,2,3$

$\mathrm{dbiw}=$

dbow $=$

$\mathrm{dbib}=$

dbvs $=$ 


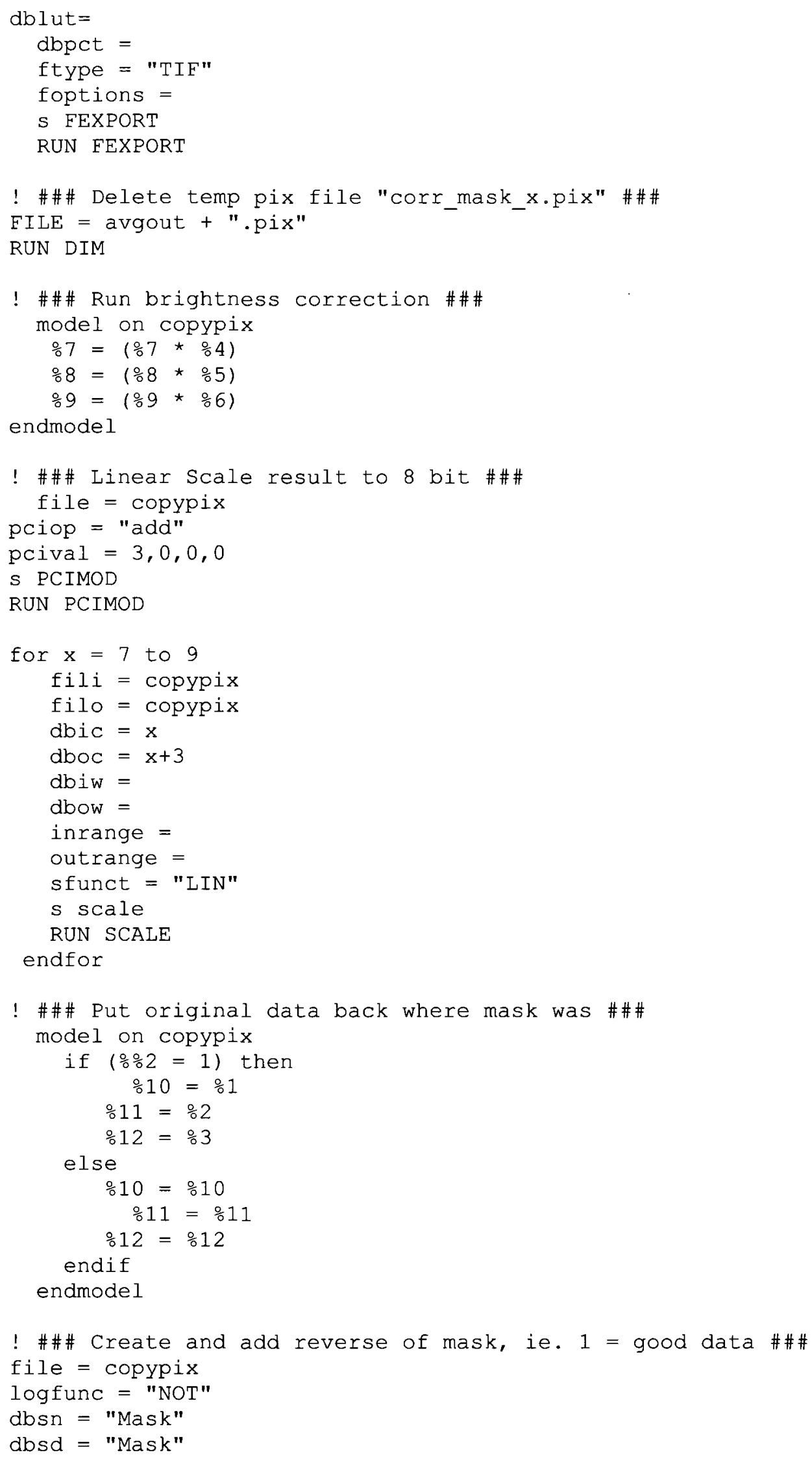




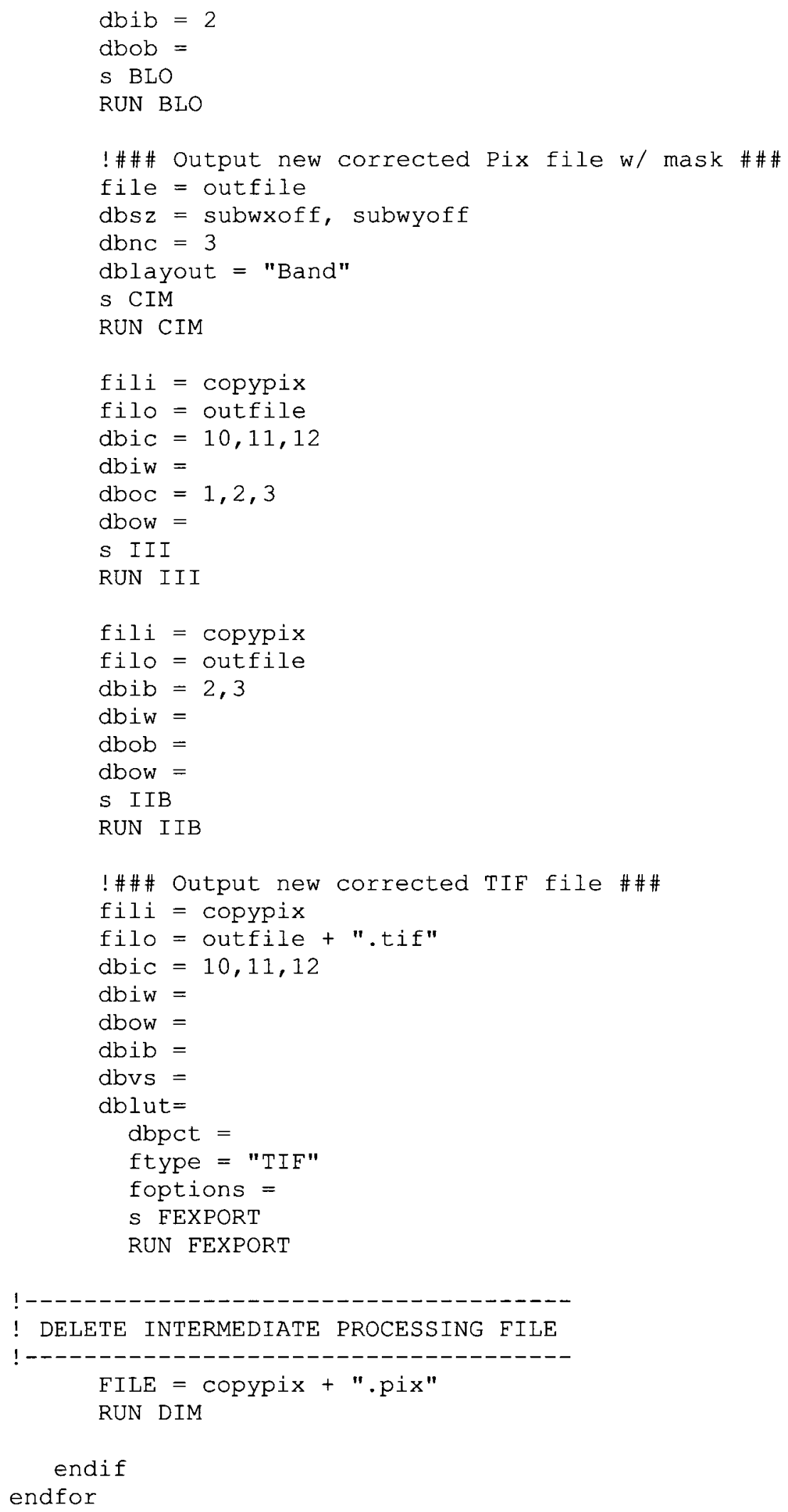


(2) The following code was written as the final step for processing the airborne imagery in order to normalize all of the corrected images (as output by the previous EASI code) and adjust the overall brightness of each image so they match that of selected "master" images. Again, each image needs a corresponding TIF mask. This code was written in Matlab r2007. It can be run by saving it as a ${ }^{*} . \mathrm{m}$ file. This algorithm is based on an algorithm provided by Nielsen (2004).

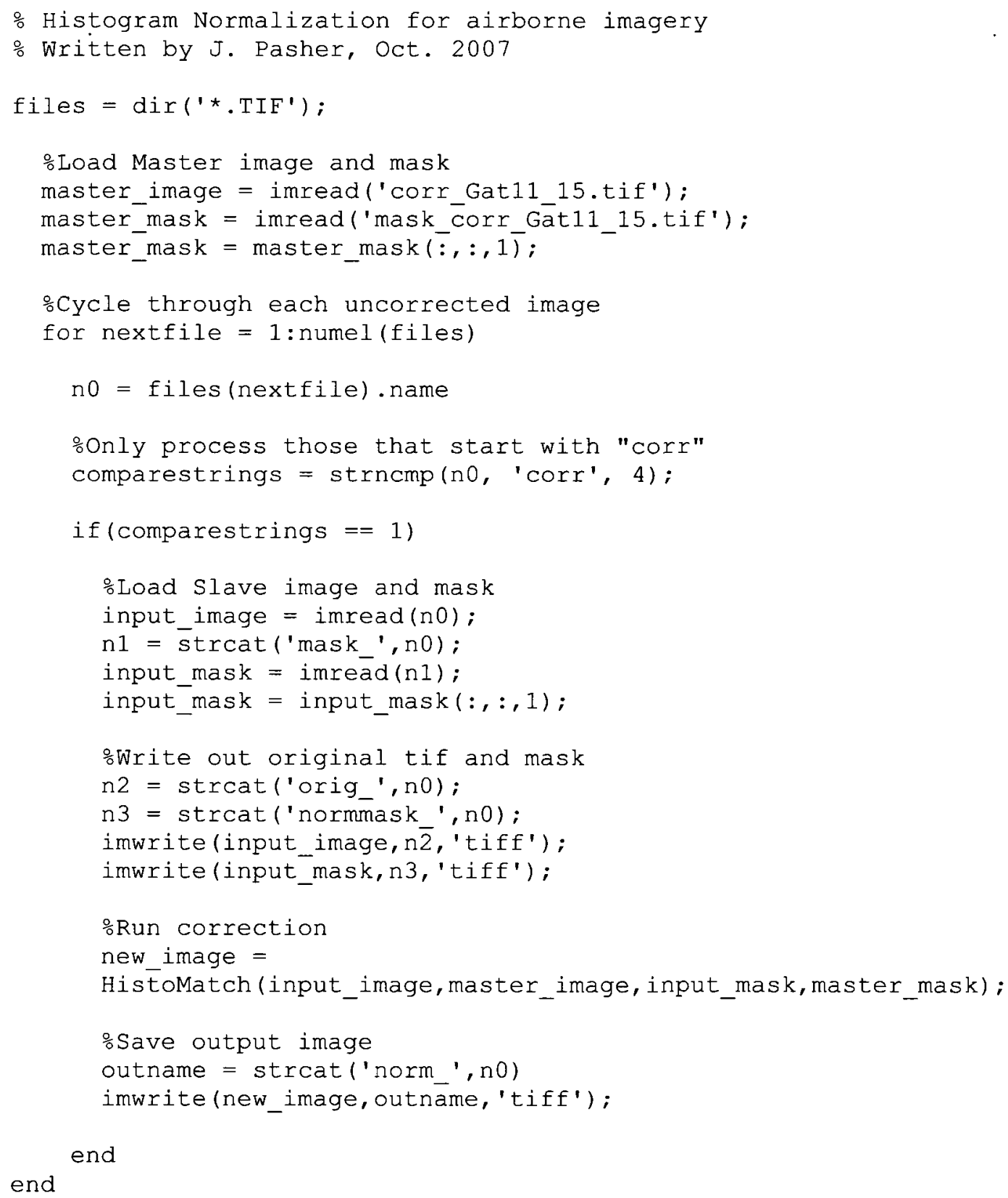




\section{APPEndiX 2: BoOtSTRAPPIng REDUNDANCy ANALYsis (RDA) ProCEDURE}

The following code carries out a bootstrapping RDA procedure within the Matlab environment (written for Matlab r2007 and can be run by saving it as a *.m file). It was written with much help from Dr. Raphael Proulx and incorporates previously existing RDA code (RDAAdjusted.m and ForwardSelectionRDA.m) written by P.R. Peres-Neto (from Peres-Neto et al., 2006) as well as statistics tools (f_AIC.m and f_stnd.m) (from FATHOM Toolbox: Jones, 2008).

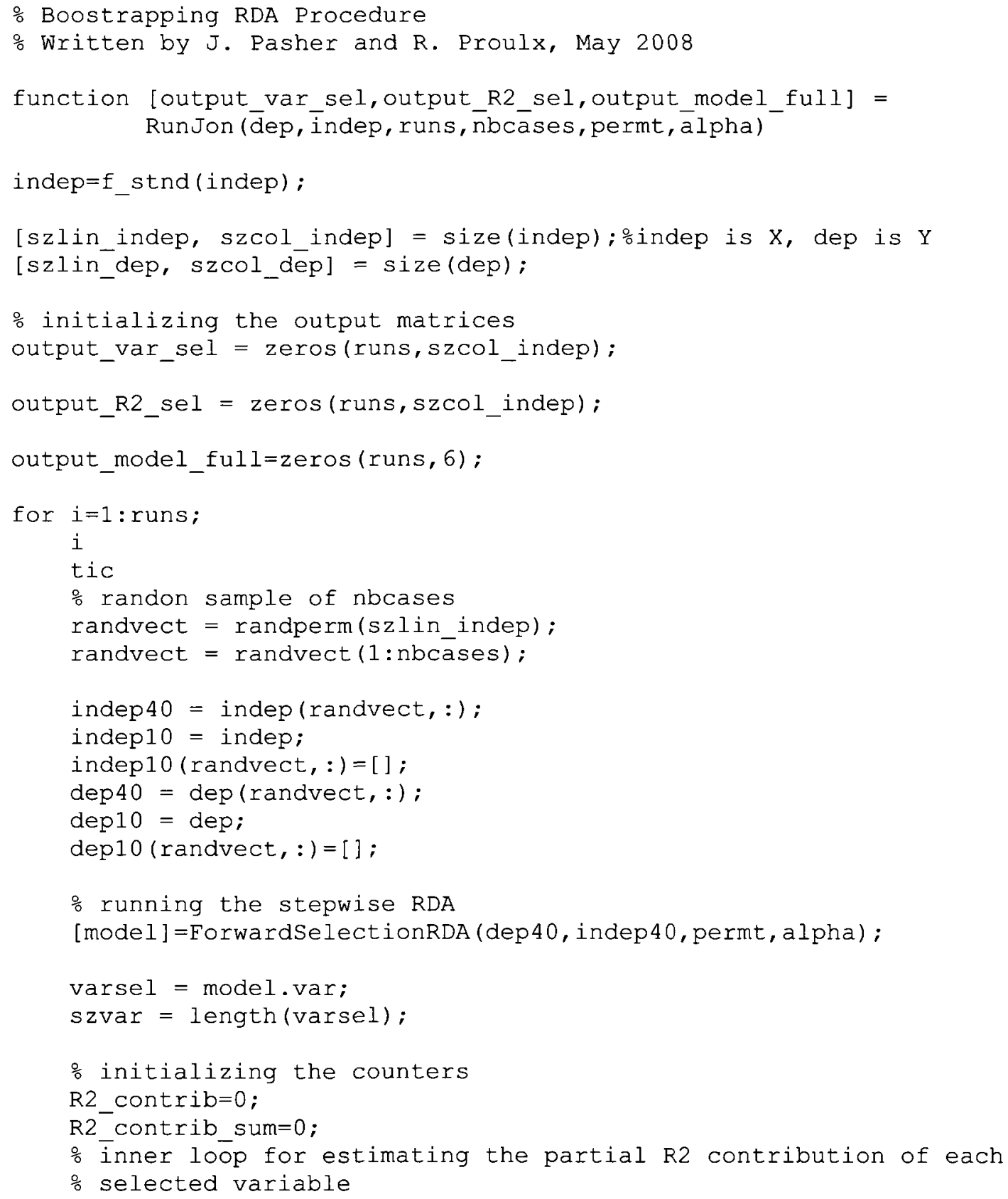




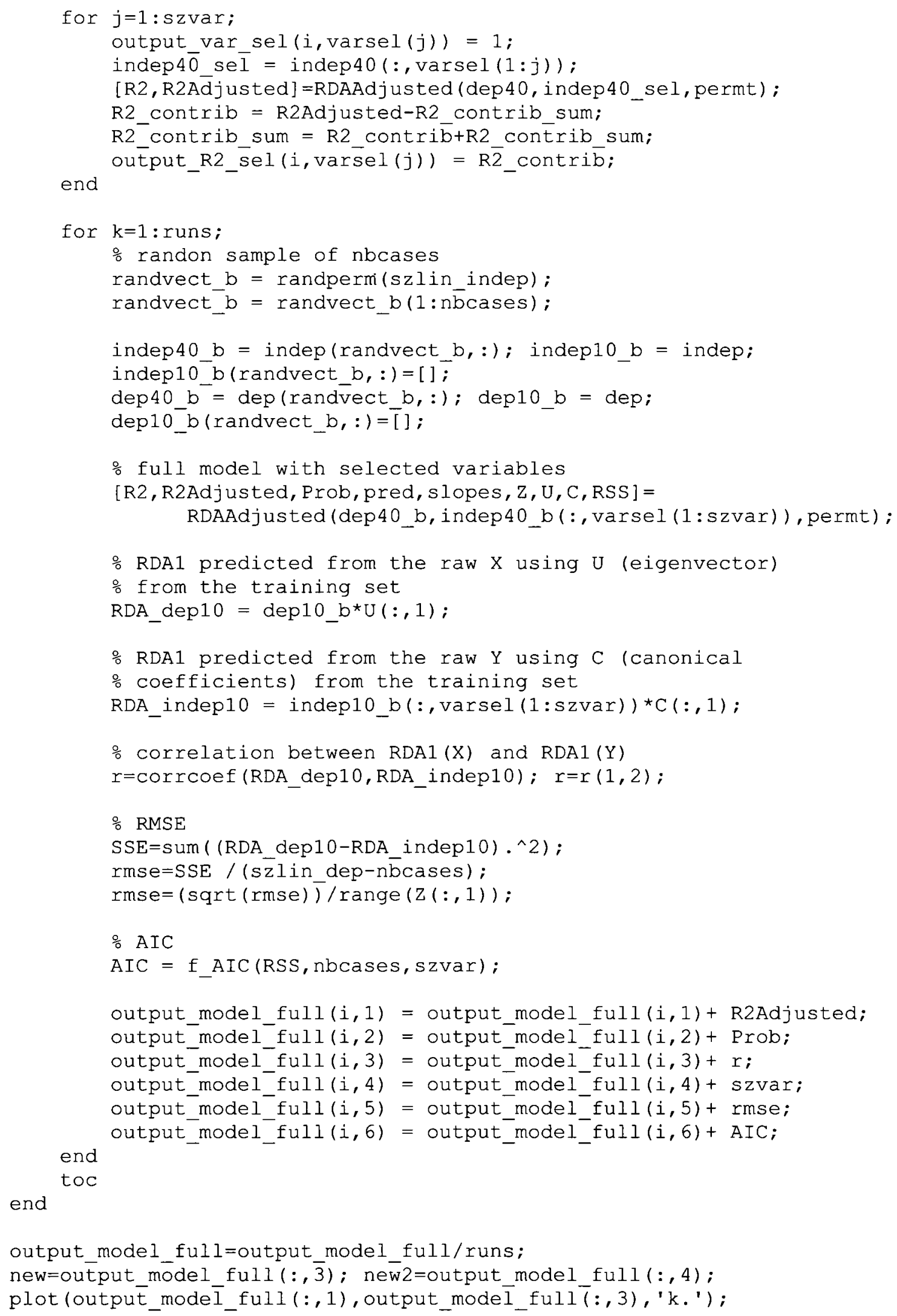


APPENDiX 3: RAW Field DATA INCLUding Plot LoCATIONS

\begin{tabular}{|c|c|c|c|c|c|c|c|}
\hline $\begin{array}{l}\text { Plot } \\
\#\end{array}$ & $\begin{array}{c}\text { Easting } \\
\text { UTM } 18 \text { NAD83 }\end{array}$ & $\begin{array}{c}\text { Northing } \\
\text { UTM } 18 \text { NAD83 }\end{array}$ & $\begin{array}{c}\text { \# of } \\
\text { Overstorey } \\
\text { Trees }\end{array}$ & $\begin{array}{l}\text { Average } \\
\text { Overstorey } \\
\text { Tree dbh } \\
\text { (cm) }\end{array}$ & $\begin{array}{l}\text { Standard } \\
\text { Deviation } \\
\text { Overstorey } \\
\text { Tree dbh } \\
\text { (cm) }\end{array}$ & $\begin{array}{c}\text { Live } \\
\text { Basal } \\
\text { Area } \\
\left(\mathrm{m}^{2} / \mathrm{ha}\right)\end{array}$ & $\begin{array}{l}\# . \\
\text { Large } \\
\text { Trees }\end{array}$ \\
\hline 1 & 428881.32768 & 5040265.84151 & 20 & 26.28 & 11.06 & 31.68 & 5 \\
\hline 2 & 428802.74861 & 5040256.95589 & 28 & 24.16 & 7.31 & 34.92 & 0 \\
\hline 3 & 428882.66620 & 5039884.08888 & 22 & 21.33 & 6.77 & 21.54 & 0 \\
\hline 4 & 428928.81184 & 5039841.44560 & 1.7 & 24.87 & 9.40 & 24.82 & 2 \\
\hline 5 & 428911.52730 & 5039764.57154 & 18 & 23.94 & 8.39 & 22.61 & 2 \\
\hline 6 & 428816.26857 & 5039727.82696 & 22 & 21.07 & 7.42 & 21.44 & 1 \\
\hline 7 & 428791.92002 & 5043621.08785 & 27 & 20.93 & 7.84 & 26.35 & 1 \\
\hline 8 & 428801.15171 & 5043687.34600 & 20 & 24.45 & 8.21 & 25.98 & 1 \\
\hline 9 & 428865.25034 & 5043570.90948 & 22 & 23.40 & 9.67 & 27.50 & 3 \\
\hline 10 & 428765.33095 & 5043570.54850 & 14 & 30.65 & 13.22 & 30.28 & 6 \\
\hline 11 & 428731.92057 & 5043459.08353 & 12 & 33.29 & 10.67 & 28.57 & 4 \\
\hline 12 & 428804.12392 & 5043507.48654 & 16 & 25.84 & 9.48 & 23.63 & 4 \\
\hline 13 & 428889.57296 & 5043479.28549 & 14 & 26.47 & 11.52 & 22.64 & 3 \\
\hline 14 & 428826.65464 & 5043426.52201 & 18 & 24.21 & 9.81 & 23.92 & 3 \\
\hline 15 & 428720.31906 & 5043372.33289 & 16 & 27.20 & 10.58 & 26.53 & 4 \\
\hline 16 & 428814.95718 & 5043359.12430 & 13 & 24.46 & 9.73 & 17.50 & 1 \\
\hline 17 & 428992.38933 & 5042546.28334 & 17 & 24.32 & 8.88 & 22.22 & 2 \\
\hline 18 & 428861.57813 & 5042526.44391 & 15 & 24.72 & 10.86 & 21.24 & 3 \\
\hline 19 & 428810.86178 & 5042508.15898 & 9 & 21.54 & 15.92 & 12.18 & 2 \\
\hline 20 & 428818.80316 & 5040769.91817 & 11 & 31.86 & 13.13 & 25.31 & 4 \\
\hline 21 & 428859.74888 & 5040704.36802 & 38 & 20.98 & 8.03 & 37.54 & 1 \\
\hline 22 & 428927.95977 & 5040580.60906 & 25 & 21.62 & 8.96 & 26.72 & 3 \\
\hline 23 & 428808.50784 & 5040572.01834 & 13 & 32.03 & 11.57 & 29.34 & 4 \\
\hline 24 & 428796.85815 & 5040535.18682 & 13 & 25.78 & 7.19 & 18.19 & 1 \\
\hline 25 & 428715.39778 & 5039424.57909 & 14 & 22.83 & 5.74 & 15.17 & 0 \\
\hline 26 & 428782.88836 & 5039511.18748 & 39 & 19.37 & 6.03 & 31.43 & 1 \\
\hline 27 & 428708.31952 & 5039682.78364 & 19 & 30.49 & 7.88 & 36.88 & 5 \\
\hline 28 & 428779.55921 & 5039634.05250 & 5 & 28.65 & 3.85 & 8.17 & 0 \\
\hline 29 & 428836.95032 & 5039653.26559 & 15 & 31.02 & 10.89 & 31.61 & 4 \\
\hline 30 & 428884.70934 & 5039496.20250 & 25 & 21.86 & 6.67 & 25.56 & 0 \\
\hline 31 & 428933.64623 & 5039478.86115 & 19 & 26.45 & 10.43 & 29.95 & 5 \\
\hline 32 & 428880.80234 & 5039621.10517 & 11 & 22.11 & 8.71 & 12.05 & 1 \\
\hline 33 & 428695.55924 & 5039742.03120 & 12 & 28.20 & 9.51 & 20.69 & 3 \\
\hline 34 & 428934.43831 & 5042590.79952 & 13 & 33.06 & 14.08 & 32.56 & 5 \\
\hline 35 & 428735.19151 & 5042488.22457 & 34 & 20.54 & 17.17 & 47.26 & 2 \\
\hline 36 & 428729.43059 & 5042365.15671 & 35 & 21.34 & 7.98 & 35.56 & 1 \\
\hline 37 & 428841.19144 & 5042391.59504 & 32 & 18.59 & 7.01 & 24.71 & 0 \\
\hline 38 & 428934.16961 & 5042373.48440 & 20 & 23.38 & 6.84 & 23.21 & 2 \\
\hline 39 & 428915.63217 & 5042309.69885 & 26 & 21.06 & 8.38 & 26.09 & 2 \\
\hline 40 & 428833.50360 & 5042332.15116 & 36 & 20.99 & 8.69 & 36.33 & 2 \\
\hline 41 & 428873.76428 & 5041286.36604 & 20 & 17.67 & 6.30 & 13.73 & 0 \\
\hline 42 & 428805.48440 & 5041203.97888 & 38 & 19.24 & 7.82 & 32.07 & 1 \\
\hline 43 & 428902.93224 & 5041444.87178 & 25 & 20.63 & 9.51 & 25.15 & 2 \\
\hline 44 & 428923.34038 & 5041501.12368 & 35 & 20.59 & 7.04 & 32.44 & 0 \\
\hline 45 & 428753.43484 & 5041532.35129 & 39 & 19.38 & 7.01 & 32.41 & 1 \\
\hline 46 & 428714.76113 & 5041531.43296 & 18 & 24.69 & 11.59 & 26.02 & 3 \\
\hline 47 & 428892.31226 & 5040478.63694 & 16 & 30.16 & 6.74 & 29.92 & 2 \\
\hline 48 & 428759.59058 & 5041340.39334 & 42 & 16.93 & 4.34 & 25.16 & 0 \\
\hline 49 & 428761.41153 & 5041449.84108 & 14 & 23.46 & 12.93 & 19.40 & 2 \\
\hline 50 & 428785.35918 & 5041385.16019 & 13 & 27.99 & 11.77 & 23.26 & 3 \\
\hline
\end{tabular}




\begin{tabular}{|c|c|c|c|c|c|c|c|c|}
\hline $\begin{array}{c}\text { Plot } \\
\#\end{array}$ & $\begin{array}{l}\text { Average } \\
\text { Nearest } \\
\text { Neighbour } \\
\text { Distance } \\
\text { (m) }\end{array}$ & $\begin{array}{c}\text { Standard } \\
\text { Deviation } \\
\text { Tree } \\
\text { Height } \\
\text { (m) }\end{array}$ & $\begin{array}{c}\text { Average } \\
\text { Crown } \\
\text { Diameter } \\
\text { (m) }\end{array}$ & $\begin{array}{c}\text { Standard } \\
\text { Deviation } \\
\text { Crown } \\
\text { Diameter } \\
\text { (m) }\end{array}$ & $\begin{array}{l}\text { \# of } \\
\text { Snags }\end{array}$ & $\begin{array}{c}\text { Average } \\
\text { Snag dbh } \\
\text { (cm) }\end{array}$ & $\begin{array}{c}\text { Dead } \\
\text { Basal } \\
\text { Area } \\
\left(\mathrm{m}^{2} / \mathrm{ha}\right)\end{array}$ & $\begin{array}{l}\text { \# of } \\
\text { Pieces } \\
\text { CWD }\end{array}$ \\
\hline 1 & 1.24 & 6.11 & 3.69 & 1.02 & 3 & 31.83 & 6.14 & 16 \\
\hline 2 & 2.11 & 4.08 & 3.73 & 1.27 & 2 & 25.31 & 2.77 & 16 \\
\hline 3 & 1.67 & 3.65 & 4.25 & 1.52 & 5 & 24.38 & 6.56 & 22 \\
\hline 4 & 2.31 & 5.17 & 4.49 & 1.68 & 4 & 35.25 & 9.87 & 21 \\
\hline 5 & 2.17 & 5.49 & 4.41 & 1.45 & 12 & 19.74 & 9.47 & 17 \\
\hline 6 & 1.72 & 4.82 & 4.28 & 1.43 & 7 & 25.37 & 9.26 & 15 \\
\hline 7 & 1.25 & 4.69 & 3.73 & 1.38 & 9 & 15.56 & 4.92 & 10 \\
\hline 8 & 1.64 & 2.90 & 4.01 & 1.27 & 8 & 16.07 & 4.49 & 19 \\
\hline 9 & 1.20 & 5.06 & 4.24 & 1.59 & 3 & 25.15 & 3.82 & 13 \\
\hline 10 & 2.89 & 5.05 & 5.40 & 1.91 & 1 & 12.41 & 0.30 & 4 \\
\hline 11 & 2.55 & 3.15 & 5.27 & 1.10 & 1 & 14.01 & 0.39 & 1 \\
\hline 12 & 1.85 & 5.87 & 3.65 & 0.97 & 9 & 22.88 & 11.73 & 18 \\
\hline 13 & 1.86 & 6.97 & 4.21 & 0.99 & 1 & 16.55 & 0.54 & 18 \\
\hline 14 & 2.14 & 3.63 & 3.53 & 0.88 & 5 & 25.46 & 7.12 & 24 \\
\hline 15 & 2.67 & 3.08 & 3.58 & 0.70 & 5 & 18.72 & 3.89 & 12 \\
\hline 16 & 2.64 & 6.05 & 3.76 & 1.00 & 1 & 28.97 & 1.65 & 9 \\
\hline 17 & 2.24 & 3.01 & 4.06 & 1.31 & 1 & 28.97 & 1.39 & 9 \\
\hline 18 & 2.15 & 5.48 & 3.76 & 1.21 & 1 & 20.37 & 0.81 & 8 \\
\hline 19 & 3.18 & 4.73 & 4.29 & 1.04 & 1 & 44.56 & 3.90 & 26 \\
\hline 20 & 1.85 & 4.78 & 3.98 & 1.19 & 4 & 30.08 & 7.37 & 12 \\
\hline 21 & 1.43 & 2.90 & 3.61 & 0.99 & 6 & 16.29 & 3.58 & 22 \\
\hline 22 & 2.94 & 4.27 & 2.94 & 0.70 & 6 & 21.80 & 5.79 & 11 \\
\hline 23 & 2.35 & 6.87 & 4.35 & 1.41 & 3 & 28.86 & 5.04 & 8 \\
\hline 24 & 1.93 & 5.31 & 3.92 & 0.83 & 7 & 21.37 & 6.62 & 16 \\
\hline 25 & 1.24 & 5.68 & 4.36 & 0.83 & 19 & 20.24 & 16.77 & 6 \\
\hline 26 & 1.41 & 3.17 & 3.31 & 0.82 & 8 & 14.76 & 3.56 & 3 \\
\hline 27 & 1.91 & 2.47 & 4.73 & 1.18 & 4 & 20.37 & 3.30 & 16 \\
\hline 28 & 2.64 & 2.33 & 4.54 & 1.14 & 3 & 49.34 & 19.15 & 20 \\
\hline 29 & 2.92 & 4.55 & 4.34 & 1.05 & 3 & 33.32 & 7.28 & 9 \\
\hline 30 & 1.46 & 4.76 & 3.95 & 0.80 & 2 & 14.64 & 0.91 & 9 \\
\hline 31 & 2.12 & 4.07 & 4.18 & 1.12 & 2 & 20.05 & 1.60 & 3 \\
\hline 32 & 2.34 & 6.96 & 3.29 & 0.07 & 4 & 25.86 & 5.33 & 30 \\
\hline 33 & 2.51 & 3.35 & 4.17 & 1.00 & 4 & 26.74 & 6.83 & 20 \\
\hline 34 & 2.39 & 5.39 & 4.61 & 2.24 & 1 & 29.92 & 1.76 & 7 \\
\hline 35 & 1.35 & 2.91 & 4.10 & 2.82 & 2 & 13.69 & 0.75 & 5 \\
\hline 36 & 1.09 & 3.47 & 4.12 & 1.12 & 4 & 17.67 & 2.78 & 7 \\
\hline 37 & 1.49 & 3.64 & 3.89 & 0.85 & 6 & 23.61 & 9.08 & 6 \\
\hline 38 & 1.72 & 3.37 & 4.04 & 0.97 & 4 & 22.20 & 6.29 & 14 \\
\hline 39 & 1.36 & 3.44 & 4.02 & 1.07 & 9 & 20.44 & 9.11 & 6 \\
\hline 40 & 1.67 & 2.50 & 3.85 & 1.03 & 3 & 12.84 & 0.97 & 6 \\
\hline 41 & 1.82 & 4.54 & 3.17 & 0.93 & 7 & 26.19 & 10.96 & 22 \\
\hline 42 & 1.09 & 2.24 & 3.61 & 1.10 & 9 & 16.30 & 5.00 & 4 \\
\hline 43 & 2.07 & 2.52 & 3.85 & 0.84 & 3 & 22.28 & 3.33 & 13 \\
\hline 44 & 1.55 & 3.08 & 3.61 & 0.99 & 5 & 11.97 & 1.44 & 7 \\
\hline 45 & 1.56 & 3.15 & 3.75 & 0.94 & 3 & 14.01 & 1.18 & 5 \\
\hline 46 & 1.82 & 2.70 & 4.30 & 1.53 & 7 & 19.87 & 6.37 & 11 \\
\hline 47 & 2.34 & 3.62 & 4.53 & 1.20 & 8 & 24.63 & 10.01 & 4 \\
\hline 48 & 0.99 & 2.54 & 3.42 & 0.95 & 7 & 19.10 & 5.24 & 11 \\
\hline 49 & 2.28 & 3.21 & 4.13 & 0.96 & 8 & 23.95 & 10.36 & 10 \\
\hline 50 & 2.35 & 5.84 & 4.50 & 1.10 & 4 & 30.16 & 7.68 & 6 \\
\hline
\end{tabular}




\begin{tabular}{|c|c|c|c|c|c|}
\hline Plot \# & $\begin{array}{c}0-10 \mathrm{~cm} \\
\text { Ground } \\
\text { Vegetation } \\
(\%)\end{array}$ & $\begin{array}{c}10-50 \mathrm{~cm} \\
\text { Ground } \\
\text { Vegetation } \\
(\%)\end{array}$ & $\begin{array}{c}50 \mathrm{~cm}-1 \mathrm{~m} \\
\text { Ground } \\
\text { Vegetation } \\
(\%)\end{array}$ & $\begin{array}{c}1-2 \mathrm{~m} \\
\text { Ground } \\
\text { Vegetation } \\
(\%)\end{array}$ & $\begin{array}{c}>2 \mathrm{~m} \\
\text { Ground } \\
\text { Vegetation } \\
(\%)\end{array}$ \\
\hline 1 & 21.43 & 15.18 & 1.79 & 0.89 & 1.79 \\
\hline 2 & 4.46 & 6.25 & 0.00 & 0.00 & 0.00 \\
\hline 3 & 12.50 & 27.68 & 8.93 & 0.00 & 0.89 \\
\hline 4 & 0.89 & 24.11 & 2.68 & 4.46 & 11.61 \\
\hline 5 & 5.36 & 19.64 & 3.57 & 1.79 & 11.61 \\
\hline 6 & 2.68 & 16.07 & 8.93 & 2.68 & 0.00 \\
\hline 7 & 9.82 & 30.36 & 7.14 & 5.36 & 8.04 \\
\hline 8 & 13.39 & 14.29 & 4.46 & 2.68 & 0.00 \\
\hline 9 & 7.14 & 55.36 & 3.57 & 5.36 & 2.68 \\
\hline 10 & 11.61 & 10.71 & 1.79 & 0.00 & 0.00 \\
\hline 11 & 0.00 & 45.54 & 7.14 & 21.43 & 18.75 \\
\hline 12 & 4.46 & 6.25 & 4.46 & 18.75 & 44.64 \\
\hline 13 & 8.04 & 50.89 & 9.82 & 8.04 & 11.61 \\
\hline 14 & 0.00 & 18.75 & 6.25 & 12.50 & 23.21 \\
\hline 15 & 9.82 & 15.18 & 0.89 & 18.75 & 32.14 \\
\hline 16 & 9.82 & 41.96 & 16.96 & 2.68 & 1.79 \\
\hline 17 & 0.89 & 17.86 & 18.75 & 13.39 & 10.71 \\
\hline 18 & 6.25 & 16.96 & 13.39 & 21.43 & 16.96 \\
\hline$\overline{19}$ & 0.00 & 25.89 & 0.00 & 0.89 & 51.79 \\
\hline 20 & 0.00 & 6.25 & 2.68 & 3.57 & 67.86 \\
\hline 21 & 3.57 & 30.36 & 9.82 & 29.46 & 2.68 \\
\hline 22 & 1.79 & 6.25 & 3.57 & 17.86 & 51.79 \\
\hline 23 & 0.00 & 4.46 & 2.68 & 2.68 & 78.57 \\
\hline 24 & 0.00 & 10.71 & 3.57 & 12.50 & 51.79 \\
\hline 25 & 17.86 & 21.43 & 33.04 & 11.61 & 0.89 \\
\hline 26 & 13.39 & 32.14 & 6.25 & 1.79 & 0.89 \\
\hline 27 & 8.04 & 28.57 & 5.36 & 3.57 & 14.29 \\
\hline 28 & 3.57 & 35.71 & 0.00 & 0.89 & 8.04 \\
\hline 29 & 5.36 & 34.82 & 2.68 & 8.93 & 36.61 \\
\hline 30 & 5.36 & 34.82 & 2.68 & 8.93 & 36.61 \\
\hline 31 & 0.00 & 37.50 & 0.89 & 20.54 & 31.25 \\
\hline 32 & 0.89 & 21.43 & 10.71 & 14.29 & 33.93 \\
\hline 33 & 0.00 & 15.18 & 0.00 & 1.79 & 25.89 \\
\hline 34 & 5.36 & 8.93 & 0.89 & 1.79 & 46.43 \\
\hline 35 & 8.93 & 59.82 & 11.61 & 0.00 & 0.89 \\
\hline 36 & 2.68 & 38.39 & 1.79 & 6.25 & 0.00 \\
\hline 37 & 1.79 & 76.79 & 2.68 & 4.46 & 5.36 \\
\hline 38 & 4.46 & 34.82 & 25.00 & 12.50 & 8.93 \\
\hline 39 & 22.32 & 22.32 & 8.04 & 8.04 & 3.57 \\
\hline 40 & 3.57 & 47.32 & 13.39 & 3.57 & 1.79 \\
\hline 41 & 0.00 & 34.82 & 10.71 & 6.25 & 23.21 \\
\hline 42 & 0.00 & 78.57 & 5.36 & 0.89 & 0.00 \\
\hline 43 & 10.71 & 25.89 & 8.04 & 5.36 & 6.25 \\
\hline 44 & 25.00 & 44.64 & 5.36 & 3.57 & 2.68 \\
\hline 45 & 8.93 & 11.61 & 1.79 & 4.46 & 1.79 \\
\hline 46 & 1.79 & 13.39 & 12.50 & 24.11 & 39.29 \\
\hline 47 & 0.89 & 2.68 & 6.25 & 21.43 & 68.75 \\
\hline 48 & 0.00 & 90.18 & 5.36 & 0.00 & 0.00 \\
\hline 49 & 2.68 & 17.86 & 10.71 & 19.64 & 26.79 \\
\hline 50 & 0.00 & 1.79 & 0.00 & 0.89 & 97.32 \\
\hline
\end{tabular}




\begin{tabular}{|c|c|c|c|c|c|c|}
\hline Plot \# & $\begin{array}{c}1 \mathrm{~m} \\
\text { LAI-CLX } \\
\left(55-60^{\circ}\right)\end{array}$ & $\begin{array}{c}1 \mathrm{~m} \\
\text { Openness } \\
\left(0-60^{\circ}\right) \\
(\%)\end{array}$ & $\begin{array}{c}6 \mathrm{~m} \\
\text { LAl-CLX } \\
\left(55-60^{\circ}\right)\end{array}$ & $\begin{array}{c}6 \mathrm{~m} \\
\text { Openness } \\
\left(0-60^{\circ}\right) \\
(\%)\end{array}$ & $\begin{array}{l}\text { Change } \\
\text { LAl-CLX } \\
\left(55-60^{\circ}\right)\end{array}$ & $\begin{array}{c}\text { Change } \\
\text { Openness } \\
\left(0-60^{\circ}\right) \\
(\%)\end{array}$ \\
\hline 1 & 5.98 & 8.10 & 3.78 & 15.30 & -36.79 & 88.89 \\
\hline 2 & 5.16 & 3.90 & 3.68 & 7.70 & -28.68 & 97.44 \\
\hline 3 & 4.42 & 11.00 & 3.42 & 13.60 & -22.62 & 23.64 \\
\hline 4 & 6.27 & 1.60 & 3.73 & 10.80 & -40.51 & 575.00 \\
\hline 5 & 4.94 & 11.50 & 3.91 & 14.40 & -20.85 & 25.22 \\
\hline 6 & 5.73 & 5.60 & 5.29 & 10.80 & -7.68 & 92.86 \\
\hline 7 & 4.68 & 6.50 & 3.73 & 14.20 & -20.30 & 118.46 \\
\hline 8 & 4.17 & 12.90 & 5.33 & 19.00 & 27.82 & 47.29 \\
\hline 9 & 6.02 & 4.80 & 5.21 & 4.80 & -13.46 & 0.00 \\
\hline 10 & 5.24 & 6.20 & 5.36 & 8.30 & 2.29 & 33.87 \\
\hline 11 & 5.01 & 6.20 & 6.08 & 3.90 & 21.36 & -37.10 \\
\hline 12 & 6.46 & 1.00 & 4.99 & 19.30 & -22.76 & 1830.00 \\
\hline 13 & 5.51 & 7.60 & 5.14 & 7.30 & -6.72 & -3.95 \\
\hline 14 & 6.84 & 3.60 & 4.91 & 22.40 & -28.22 & 522.22 \\
\hline 15 & 6.39 & 1.00 & 4.71 & 11.30 & -26.29 & 1030.00 \\
\hline 16 & 4.49 & 13.60 & 5.35 & 13.30 & 19.15 & -2.21 \\
\hline 17 & 4.66 & 8.20 & 5.18 & 8.70 & 11.16 & 6.10 \\
\hline 18 & 5.87 & 7.60 & 3.84 & 21.40 & -34.58 & 181.58 \\
\hline 19 & 6.89 & 1.80 & 4.57 & 20.50 & -33.67 & 1038.89 \\
\hline 20 & 6.20 & 1.30 & 3.47 & 20.70 & -44.03 & 1492.31 \\
\hline 21 & 6.10 & 2.50 & 4.89 & 4.10 & -19.84 & 64.00 \\
\hline 22 & 6.67 & 2.50 & 5.34 & 12.10 & -19.94 & 384.00 \\
\hline 23 & 6.19 & 1.40 & 4.03 & 11.90 & -34.89 & 750.00 \\
\hline 24 & 6.57 & 1.60 & 2.61 & 19.40 & -60.27 & 1112.50 \\
\hline 25 & 5.36 & 14.50 & 3.23 & 24.40 & -39.74 & 68.28 \\
\hline 26 & 4.63 & 6.70 & 5.38 & 7.20 & 16.20 & 7.46 \\
\hline 27 & 4.80 & 3.30 & 4.35 & 7.20 & -9.38 & 118.18 \\
\hline 28 & 6.43 & 1.50 & 4.71 & 29.50 & -26.75 & 1866.67 \\
\hline 29 & 4.55 & 10.40 & 3.97 & 12.20 & -12.75 & 17.31 \\
\hline 30 & 4.25 & 13.50 & 3.76 & 23.90 & -11.53 & 77.04 \\
\hline 31 & 6.45 & 2.10 & 3.43 & 14.00 & -46.82 & 566.67 \\
\hline 32 & 6.13 & 3.70 & 4.10 & 31.40 & -33.12 & 748.65 \\
\hline 33 & 6.10 & 1.60 & 5.16 & 22.50 & -15.41 & 1306.25 \\
\hline 34 & 5.73 & 2.30 & 2.58 & 22.90 & -54.97 & 895.65 \\
\hline 35 & 5.39 & 4.70 & 5.38 & 4.70 & -0.19 & 0.00 \\
\hline 36 & 5.42 & 3.60 & 5.73 & 3.30 & 5.72 & -8.33 \\
\hline 37 & 5.34 & 5.00 & 5.32 & 5.40 & -0.37 & 8.00 \\
\hline 38 & 5.63 & 2.40 & 5.10 & 12.50 & -9.41 & 420.83 \\
\hline 39 & 3.06 & 11.30 & 3.07 & 15.00 & 0.33 & 32.74 \\
\hline 40 & 4.42 & 6.80 & 4.37 & 10.00 & -1.13 & 47.06 \\
\hline 41 & 4.32 & 8.40 & 2.63 & 45.50 & -39.12 & 441.67 \\
\hline 42 & 4.02 & 9.70 & 3.46 & 13.40 & -13.93 & 38.14 \\
\hline 43 & 6.65 & 9.00 & 4.85 & 29.30 & -27.07 & 225.56 \\
\hline 44 & 4.07 & 9.60 & 4.72 & 7.40 & 15.97 & -22.92 \\
\hline 45 & 3.99 & 7.90 & 3.42 & 12.10 & -14.29 & 53.16 \\
\hline 46 & 6.19 & 8.00 & 4.14 & 24.40 & -33.12 & 205.00 \\
\hline 47 & 6.19 & 6.50 & 2.42 & 32.40 & -60.90 & 398.46 \\
\hline 48 & 4.40 & 7.90 & 3.78 & 16.00 & -14.09 & 102.53 \\
\hline 49 & 4.04 & 7.00 & 3.91 & 13.40 & -3.22 & 91.43 \\
\hline 50 & 6.68 & 1.30 & 2.95 & 25.10 & -55.84 & 1830.77 \\
\hline
\end{tabular}


APPENDIX 4: SCATTERPLOTS SHOWING RELATIONSHIPS OF FIELD VARIABLES WITH THE SCI. LINE OF BEST FIT IS INCLUDED, WITH PLOTS DISPLAYED IN AN ORDER THAT CORRESPONDS TO THE CORRELATION COEFFICIENTS PRESENTED IN TABLE 7.
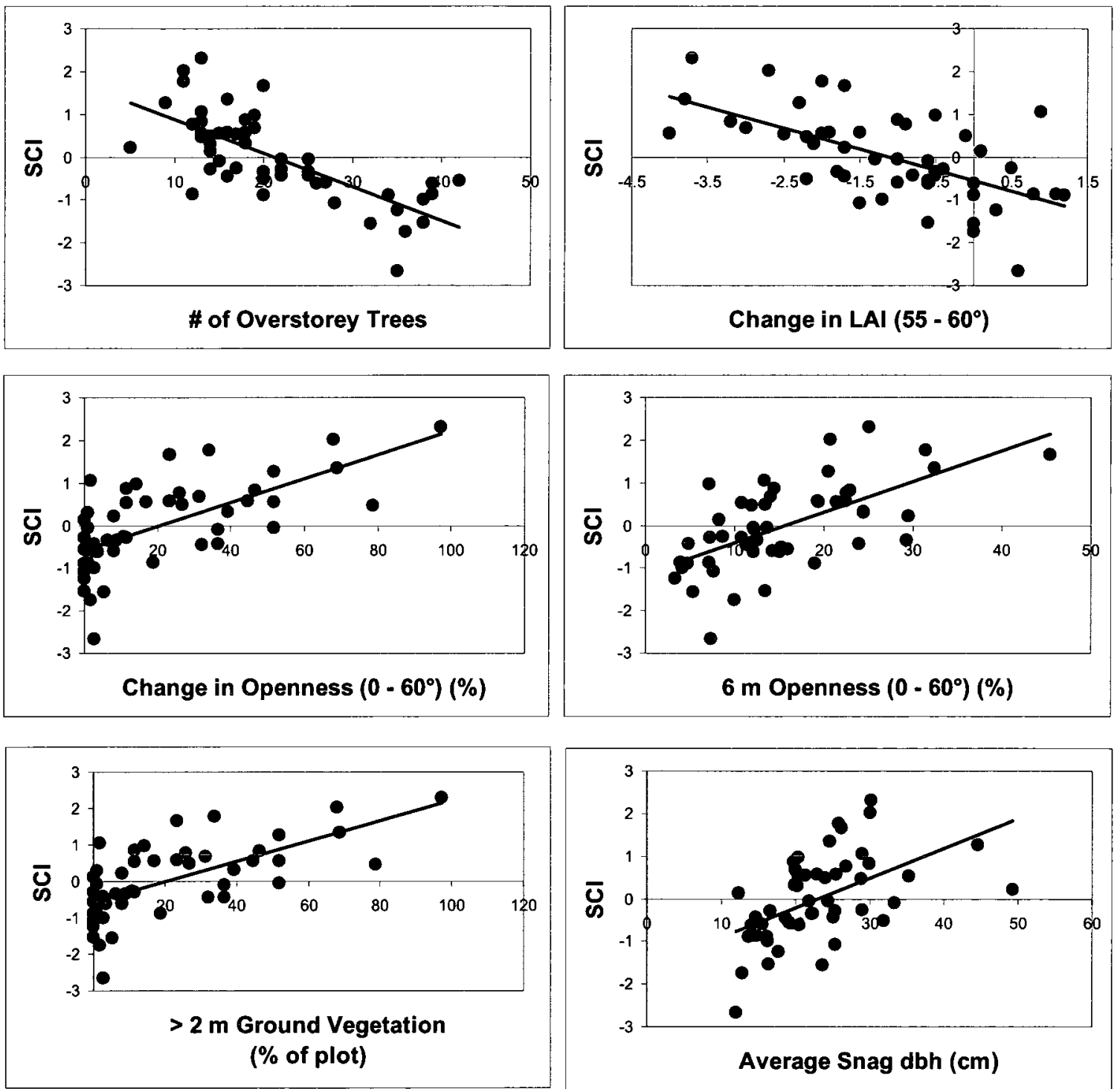

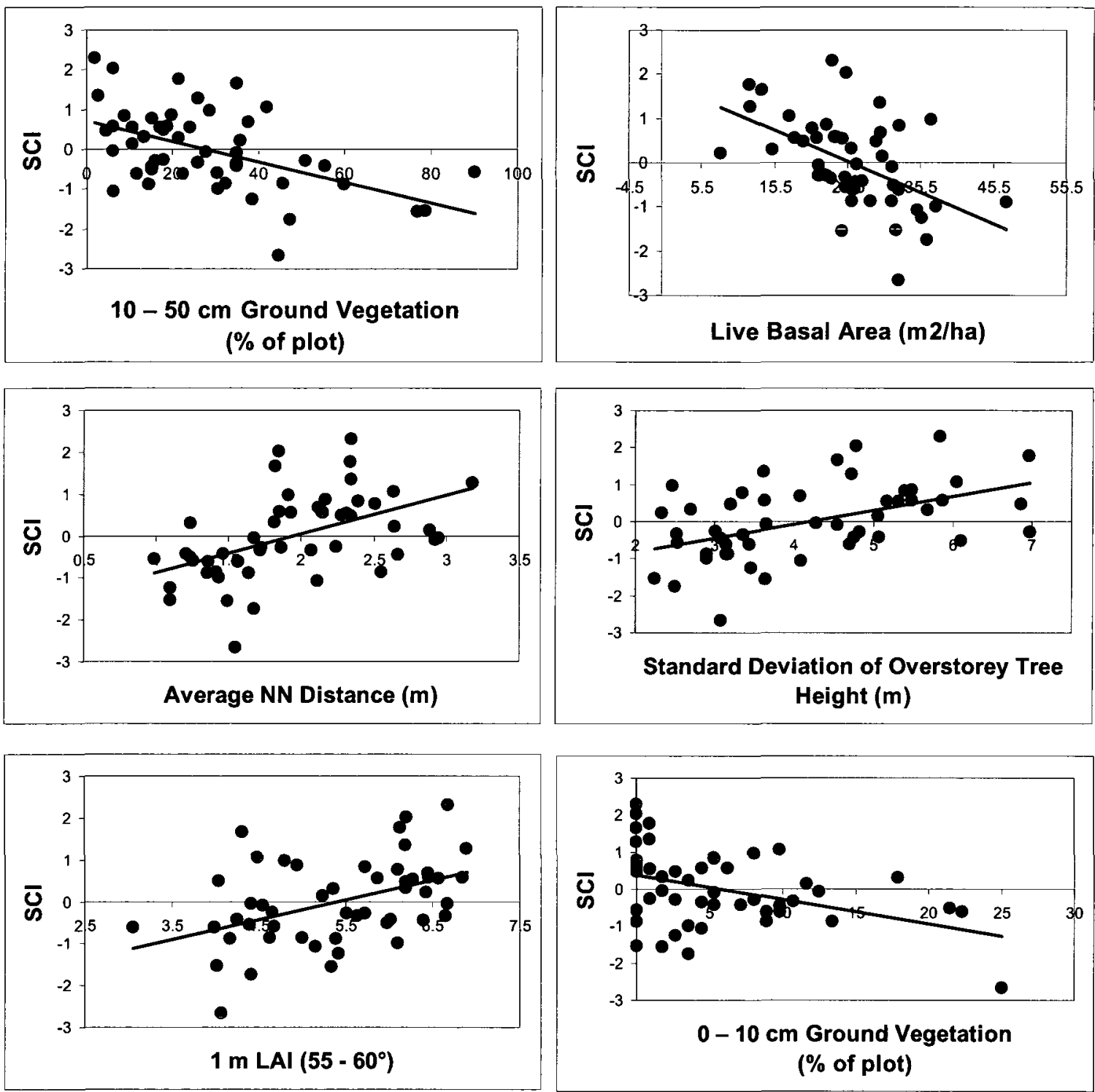

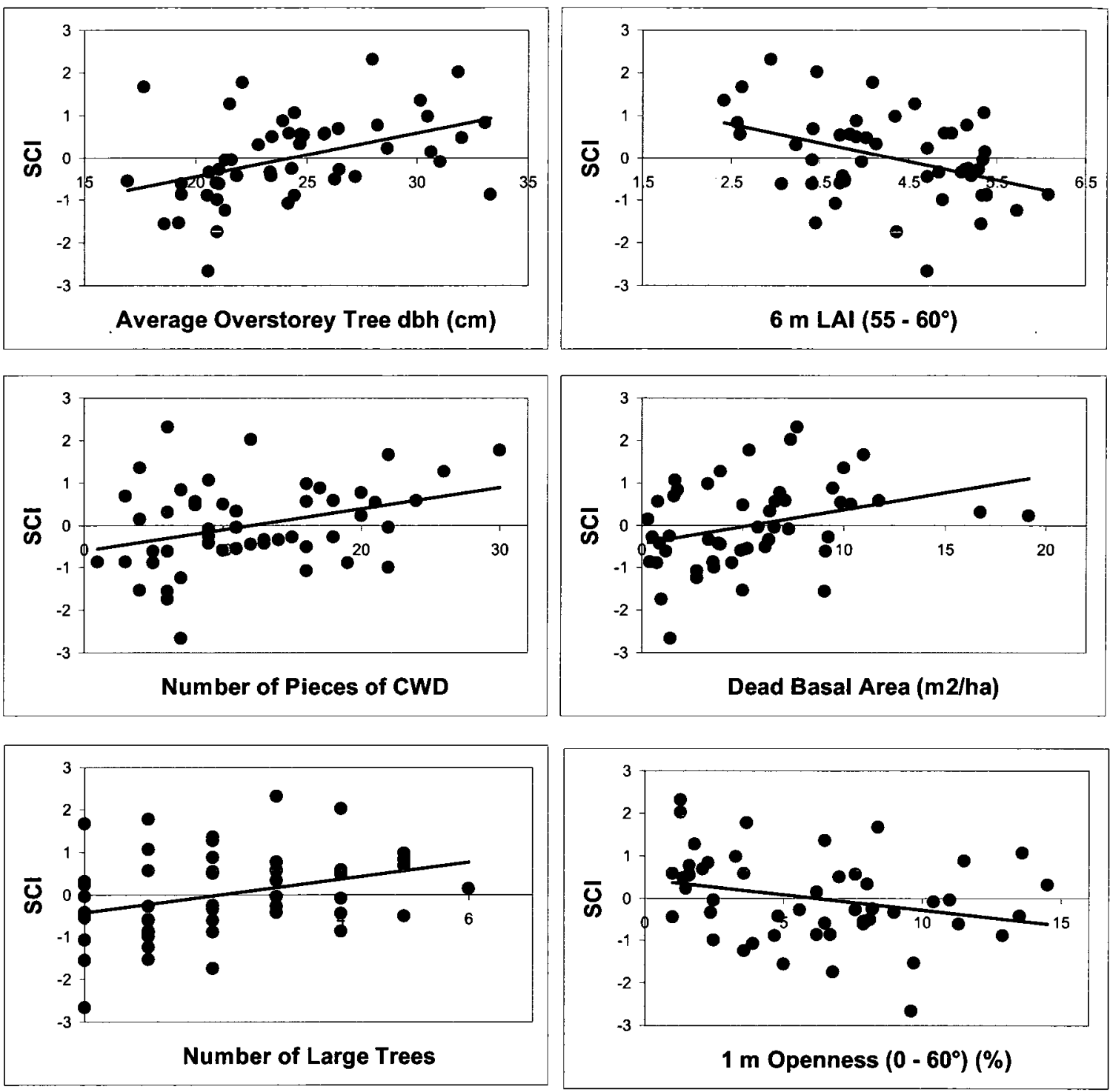

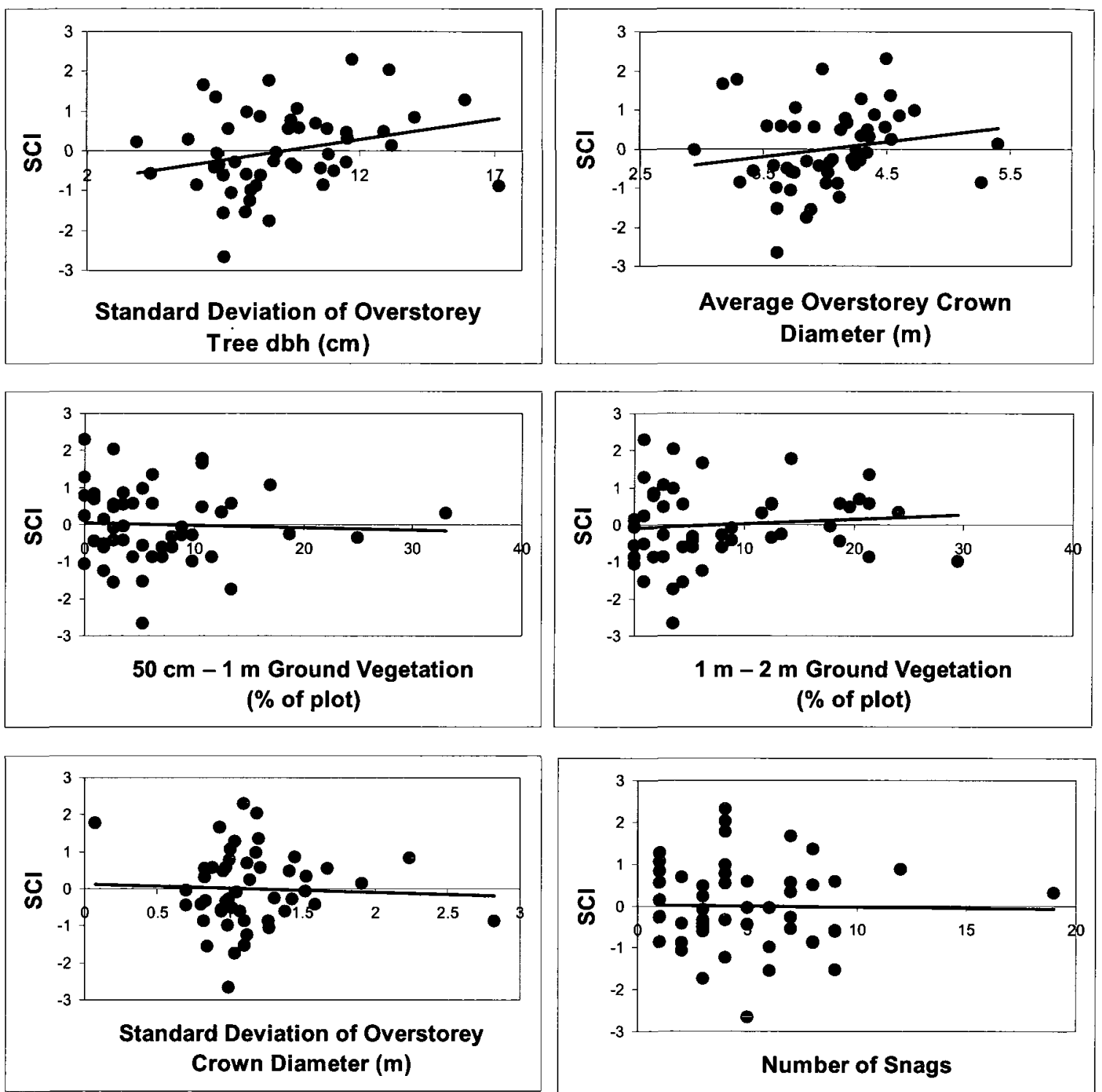